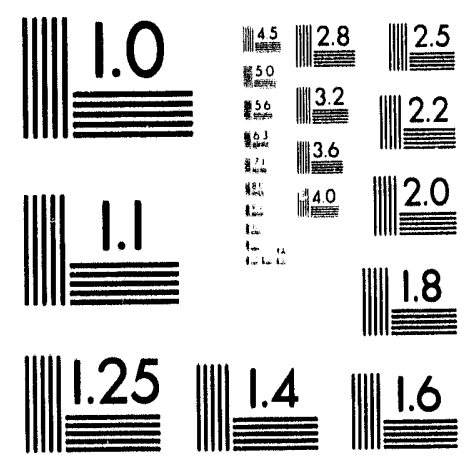



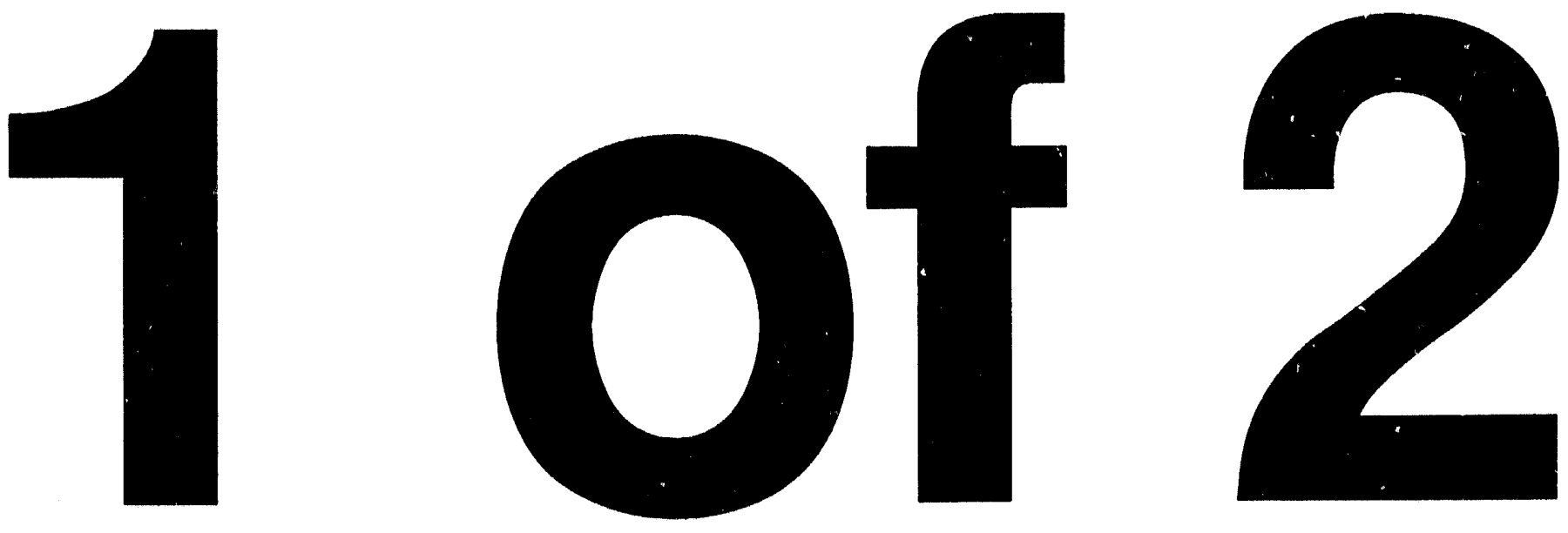


\title{
TEMPERATURE DEPENDENCE OF CSI(TI) GAMMA-RAY EXCITED SCINTILLATION CHARACTERISTICS
}

\author{
by \\ John David Valentine \\ A dissertation submitted in partial fulfillment \\ of the requirements for the degree of \\ Doctor of Philosophy \\ (Nuclear Engineering) \\ in The University of Michigan \\ 1993

\section{DISCLAIMER}

\begin{abstract}
This report was prepared as an account of work sponsored by an agency of the United States Government. Neither the United States Government nor any agency thereof, nor any of their employees, makes any warranty, express or implied, or assumes any legal liability or responsibility for the accuracy, completeness, or usefulness of any information, apparatus, product, or process disclosed, or represents that its use would not infringe privately owned rights. Reference herein to any specific commercial product, process, or service by trade name, trademark, manufacturer, or otherwise does not necessarily constitute or imply its endorsement, recommendation, or favoring by the United States Government or any agency thereof. The views and opinions of authors expressed herein do not necessarily state or reflect those of the United States Government or any agency thereof.
\end{abstract}

Doctoral Committee:

Associate Professor David K. Wehe, Chairman

Professor Ronald F. Fleming

Prciessor Glenn F. Knoll

Professor W. Leslie Rogers

Calvin E. Moss, Staff Member, Los Alamos National Laboratory 
John David Valentine
All Rights Reserved

1993

The Goverment reserves for itself and others acting on its behalf a royalty free, nonexclusive, irrevocable, world-wide license for goverrmental purposes to publish, distribute, translate, duplicate, exhibit, and perform any such data copyrighted by the contractor. 
For my wife,

Suzanne Noel Valentine

and

my parents,

Allen and Judith Valentine. 


\section{ACKNOWLEDGEMENTS}

I would like to express my gratitude to the many people who have contributed to this work. In particular, the invaluable assistance of the following people has made this quest a reality. The support, patience, and guidance of Professor David K. Wehe has molded an engineer into a research scientist. Professor Glenn F. Knoll had the original idea of measuring the energy resolution temperature dependence of $\mathrm{CsI}(\mathrm{Tl})$ coupled to a silicon PIN photodiode and has consistently guided my research toward achievahle and meaningful goals. Additionally, Professor Ronald. F. Fleming has provided considerable advice and guidance regarding the statistical treatment of centroid and full-width at half maximum uncertainty determinations.

The initial CsI(T1)/photodiode experiments were performed at Los Alamos National Laboratory (LANL) under the guidance of Dr. Calvin E. Moss, as was my initial exposure to radiation detection and measurements. I consider Dr. Moss my mentor and wish to express special gratitude to him for piquing my interest in gamma-ray detectors and exposing me to the diversity of applications for which radiation measurements can he used. I would also like to thank the people of the Advanced Nuclear Technology Group (N-2) and the Space Astronomy Group (SST-9) at LANL. Their support and guidance has contributed significantly to my growth as a nuclear engineer.

I am likewise indebted to Dr. William W. Moses and Dr. Stephen E. Derenzo of Lawrence Berkeley Laboratory (LBL). The bulk of the results presented in this dissertation are from measurements performed at LBL using the equipment that Dr. Moses and Dr. Derenzo have assembled for their scintillator search. Dr. Moses was of particular assistance in acclimating me to their equipment and helping me with the acquisition and 
analysis of the data. Dr. Moses' assistance has extended beyond what could be asked of him as he has performed additional measurements after my departure to substantiate our findings and has been kind enough to review this work for technical content. The fitting program used to determine the rise and decay components of CsI(Tl) was written and modified for my use by Dr. Derenzo. This considerable effort is very much appreciated and has allo"'ed the extensive study of the CsI(Tl) luminescence mode temporal behavior.

Many fellow graduate students have made significant contributions to this work and to my education. In particular, I would like to thank Valentin Jordanov for his help with the implementation of the shaped square wave method and for his extensive discussions regarding nuclear instrumentation, and Timothy DeVol for his invaluable discussions and insight regarding scintillators and gamma-ray detection. The scintillator research that Tim and I have performed separately has been successful because of our joint efforts involving the analysis and interpretation of our data. Additionally, I would like to thank Dr. Ruvin Deich for sharing his knowledge of the solid state physics that govern the scintillation process in $\mathrm{CsI}(\mathrm{Tl})$.

Finally, I would like to thank my friends and family for all of their support and understanding. To my wife Suzanne, whose love and support has made this dream a reality and whose patience and resilience through this process was unflappable, I owe a lifetime of the same. And to my parents, Allen and Judith Valentine, who gave me the ability and the desire to succeed in life and excel at living, I will always be indebted and gracious.

The research was performed under appointment to the Nuclear Engineering and Health Physics Fellowship Program administered by Oak Ridge Institute for Science and Education (ORISE) for the U.S. Department of Energy. The work performed at The University of Michigan was supported in part by the U.S. Department of Energy under contract No. DE-FG02-86NE37969, while the work performed at Lawrence Berkeley Laboratory was supported in part by the U.S. Department of Energy under contract No. 
DE-AC03-76SF00098 and in part by the National Heart, Lung, anu Blood Institute, National Institute of Health, and National Cancer Institute under grants No. P01-HL25840 and No. R01 CA48002. 
DEDICATION........................................................

ACKNOWLEDGEMENTS $\ldots \ldots \ldots \ldots \ldots \ldots \ldots \ldots \ldots \ldots \ldots \ldots \ldots \ldots \ldots$ iii

LIST OF TABLES ............................................... viii

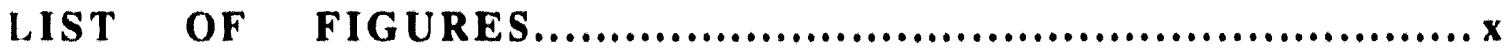

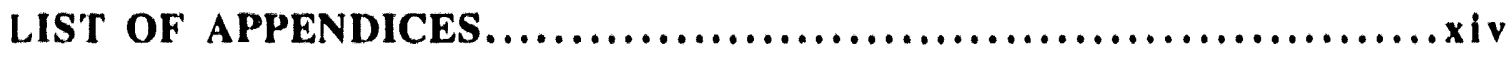

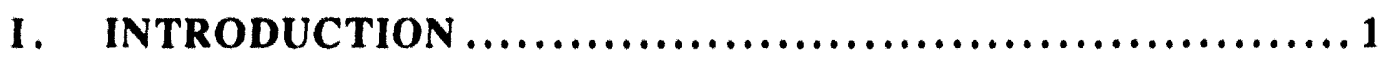

1.1 Scintillation Mechanisms .......................................... 4

1.1.1 Thallium Luminescence Centers.............................6

1.1.2 F Center Luminescence............................... 7

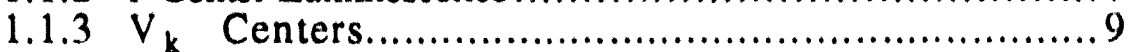

1.1.4 Intraband Luminescence.......................................10

1.2 Scintillation Characteristics that Affect Detector Performance ......12

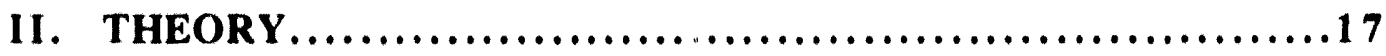

2.1 CsI(Tl) Scintillation Pulses........................................17

2.1.1 Light Collection Efficiency, $\eta(\lambda, T, t) \ldots \ldots \ldots \ldots \ldots \ldots \ldots . \ldots 19$

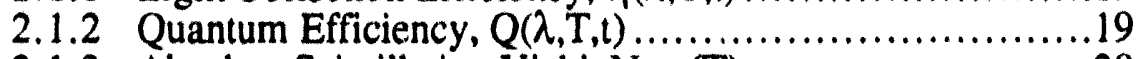

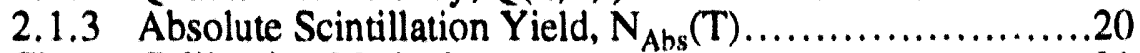

2.2 Charge Calibration Methods .....................................21

2.2.1 Direct Interactions ....................................24

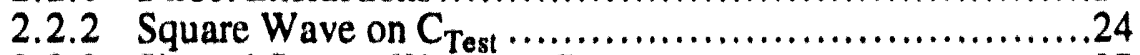

2.2.3 Shaped Square Wave on $C_{\text {Test }} \ldots \ldots \ldots \ldots \ldots \ldots \ldots \ldots \ldots \ldots . . .27$

2.3 Rise and Decay Time Constant Determination .......................30

2.3.1 Modified Bollinger-Thomas Method.......................30

2.3.2 Shaped Square Wave Method..................................33

III. EMISSION SPECTRUM..............................34

3.1 Emission Spectrum Experimental Methods .......................36

3.2 Emission Spectrum Results ............................................41

3.3 Teinporal Dependence of Emission Spectrum ......................47

IV. SCINTILLATION YIELD .............................50

4.1 $\mathrm{N}_{\mathrm{cc}}\left(\mathrm{E}_{\mathrm{r}} \mathrm{T}\right)$ as Determined by Shaped Square Wave (SSW)

Method..................................................................51

4.1.1 SSW Circuit and Method Confirmation ....................51

4.1.2 SSW Method Experimental Methods ...........................55

4.1.3 $\mathrm{N}_{c c}\left(\mathrm{E}_{\mathrm{r}} \mathrm{T}\right)$ Results from SSW Method ..........................59

4.2 Light Collection Efficiency, $\eta$...................................61

4.3 Wavelength-Averaged Quantum Efficiency .........................65

4.4 Relative Scintillation Yield Temperature Dependence as

Determined by Current Mode (CM) Method .......................70

4.4.1 CM Method Experimental Methods .......................70 
4.4.2 CM Method Results..................................72

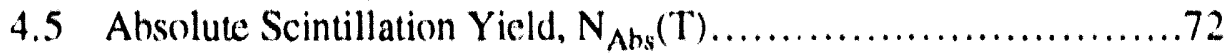

V. RISE AND DECAY TIME CONSTANTS....................84

5.1 Experimental Methods .....................................85

5.1.1 Modified Bollinger-Thomas Method..................85

5.1.2 Shaped Square Wave Method..............................87

5.2 Results....................................................88

5.2.1 Modified Bollinger-Thomas Method Results .............88

5.2.2 Shaped Square Wave Method Results ...................95

5.2.3 Comparison of MBT and SSW' Results ................97

V1. THERMOLUMINESCENCE........................ 10 2

6.1 Thermoluminescence Experimental Methods ...................1(1)4

6.2 Thermoluminescence Results ............................. 105

VII. CONCLUSIONS................................... 111

7.1 Future Work ........................................116

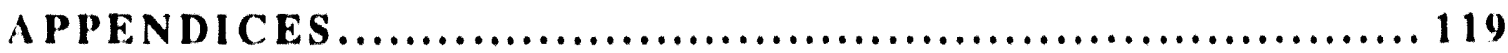

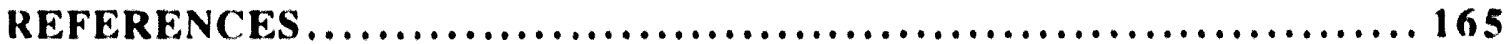




\section{LIST OF TABLES}

\section{Table}

1.1 Published values of CsI(Tl) primary decay time constants at room temperature

3.1 Room temperature internal and external wavelength-averaged quantum efficiencies for a typical silicon PIN photodiodes coupled to CsI(TI) crystals from Bicron Corporation, Horiba Crystal Products, Rexon Components, Inc., and Solon Technologies, Inc.

3.2 Temperature dependence of wavelength-averaged quantum efficiency for a typical silicon PIN photodiode coupled to the Solon Technologies, Inc. CsI(TI) crystal

4.1 Comparison of charge calibration methods

4.2 Temperature dependence of electron-hole pair yield per unit energy deposited as determined by the shaped square wave method for Solon Technologies, Inc. CsI(TI) crystal

4.3 Photomultiplier tube output current as a function of temperature for Solon Technologies, Inc. CsI(Tl) crystal.

4.4 $\mathrm{CsI}(\mathrm{TI})$ absolute scintillation yield temperature dependence as determined by the shaped square wave (SSW) method and the current mode (CM) method for the Solon Technologies, Inc. crystal. The CM method data are normalized to the $20^{\circ} \mathrm{C}$ yield determined by the SSW method

4.5 Comparison of room temperature absolute scintillation yield determined in this study to the results of Bird, et al. [BIR92] and Holl, et al. [HOL88] by correcting for differences in light collection efficiency and ballistic deficit experienced in the direct interaction (DI) calibration method for an amplifier shaping time of $3 \mu \mathrm{s}$

5.1 Comparison of rise and decay components for CsI(Tl) crystals from Bicron Corporation, Horiba Crystal Products, Rexon Components, Inc., and Solon Technologies, Inc. at room temperature and $0^{\circ} \mathrm{C}$ as determined by modified Bollinger-Thomas method....

5.2 Temperature dependence of rise and decay components for Solon Technologies, Inc. CsI(TI) crystal as determined by modified Bollinger-Thomas method. 
5.3 Temperature dependence of primary decay time constants and charge contribution ratio for Solon Technologies, Inc. CsI(TI) crystal as determined by the shaped square wave method...

C.1 Radiant sensitivity of calibrated Hamamatsu S359()-0.3 silicon PIN photodiode at room temperature...

D.1 Calculated reduced $\chi^{2}$ values for temporal luminescence intensity least squares fits... 


\section{LIST OF FIGURES}

\section{Eigure}

1.1 Comparison of emission spectra of $\mathrm{NaI}(\mathrm{Tl})$ and $\mathrm{CsI}(\mathrm{Tl})$ and quantum efficiencies of a S-11 photocathode (PMT) and a silicon PIN photodiode (PD). Emission spectra normalized to $\mathrm{NaI}(\mathrm{Tl})$ peak emission

1.2 Schematic of scintillation process …...................................... 4

1.3 Three interband luminescence processes that are typically used to explain the emission of photons by scintillators

1.4 Diagram of $\mathrm{F}$ centers in $\mathrm{CsI}(\mathrm{Tl})$....................................... 8

1.5 Schematic of luminescence process at a $\mathrm{F}$ center ....................... 8

1.6 Diagram of $\mathrm{V}_{\mathrm{k}}$ center in $\mathrm{CsI}(\mathrm{Tl})$ with possible directions for $90^{\circ}$ and

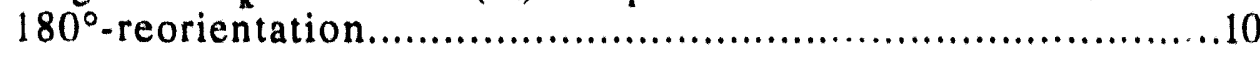

1.7 Schematic of intraband and cross luminescence along with competing phonon process for intraband luminescence

2.1 CsI(Tl) scintillation pulse shapes: (a) scintillation yield, (b) preamplifier input current pulse, (c) preamplifier output voltage pulse, and (d) amplifier output voltage pulse

2.2 Direct interaction (DI) method pulse shapes:(a) preamplifier input current pulse, (b) preamplifier output voltage pulse, and (c) amplifier output voltage pulse.

2.3 Square wave (SW) method pulse shapes: (a) calibration voltage pulse, (b) preamplifier input current pulse, (c) preamplifier output voltage pulse, and (d) amplifier output voltage pulse

2.4 Shaped square wave (SSW) method schematic.

2.5 Shaped square wave (SSW) method pulse shapes: (a) calibration voltage pulse, (b) preamplifier input current pulse, (c) preamplifier output voltage pulse, and (d) amplifier output voltage pulse.

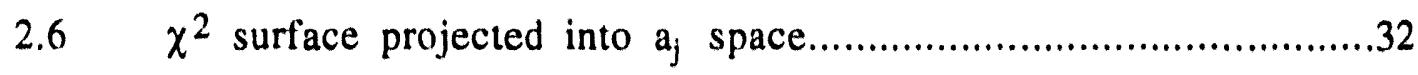

3.1 Detection system schematic for emission spectra measurement...............37 


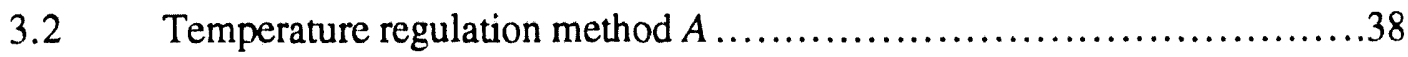

3.3 Detection system and electronics schematic for emission spectra measurements

3.4 Monochromator and PMT spectral efficiency calibration for the 300 and $500 \mathrm{~nm}$ gratings

3.5 Relative spectral efficiency of monochromator and photomultiplier tube for 300 and $500 \mathrm{~nm}$ gratings

3.6 Example of background-subtracted and background-subtracted, efficiency-corrected emission spectra data for the 300 and $500 \mathrm{~nm}$ gratings

3.7 Gamma-ray excited CsI(Tl) emission spectrum at room temperature for crystals from Bicron Corporation, Horiba Crystal Products, Rexon Components, Inc., and Solon Technologies, Inc.

3.8 Temperature dependence of gamma-ray excited CsI(TI) emission spectrum for Solon Technologies, Inc. crystal .....

4.1 Shaped square wave method circuit diagram

4.2 Normalized CsI(Tl) photopeak and charge calibration peak centroid positions as a function of amplifier shaping time constant ................54

4.3 CsI(Tl) crystal and silicon PIN photodiode schematic...................56

4.4 Configuration to complete Faraday cage around $\mathrm{CsI}(\mathrm{Tl}) /$ photodiode

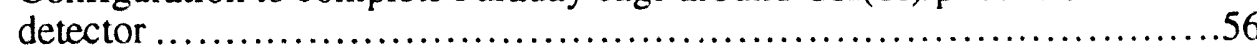

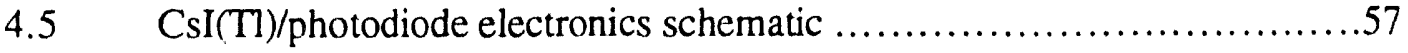

4.6 Examples of matching $\mathrm{CsI}(\mathrm{Tl})$ photopeak centroids with shaped square wave (SSW) method calibration pulse peak centroids for Solon Technologies, Inc. crystal.

4.7 Amplifier shaping time dependence of $\mathrm{CsI}(\mathrm{Tl})$ pulse height for crystals from Bicron Corporation, Horiba Crystal Products, Rexon Components, Inc., and Solon Technologies, Inc. Normalized to the $12 \mu$ s pulse height of the STI crystal.

4.8 Variation of light collection efficiency with scintillation photon wavelength due to the change in the index of refraction for silicon

$4.9 \quad$ Schematic for photodiode calibration ................................67

4.10 External and internal (quantum efficiency of calibrated Hamamatsu S3590-03 silicon PIN photodiode.

4.11 Detection system schematic for current mode method measurements......71 
4.12 Detection system and electronics schematic for current mode method measurements.......................................................71

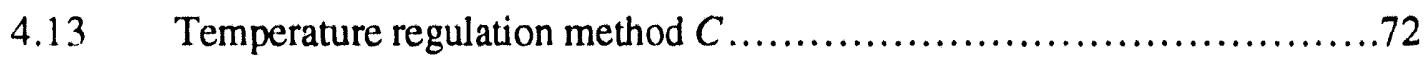

4.14 Temperature dependence of CsI(Tl) relative scintillation yield for crystals from Solon Technologies, Inc., Bicron Corporation, Horiba Crystal Products, and Rexon Components, Inc., as measured by the current mode $(\mathrm{CM})$ method

4.15 CsI(TI) absolute scintillation yield temperature dependence for Solon Technologies, Inc. crystal, as determined by the shaped square wave (SSW) method and the current mode (CM) method.

4.16 Comparison of shaped square wave (SSW) method and current mode (CM) method absolute scintillation yield results with $\mathrm{CM}$ method results corrected for response of Hamamatsu R-2059 (CM corrected) . ....77

4.17 Temperature dependence of $\mathrm{CsI}(\mathrm{Tl})$ pulse height for amplifier shaping time constants of 2,5 , and $12 \mu \mathrm{s}$.

4.18 Previously reported CsI(T1) scintillation yield temperature dependence, measured using a single amplifier shaping time, compared to $2 \mu$ s pulse height temperature dependence from this study

5.1 Detector geometry for modified Bollinger-Thomas method measurements.

5.2 Electronics schematic for modified Bollinger-Thomas method measurements

5.3 Gamma-ray excited CsI(Tl) luminescence timing spectra at room temperature for Solon Technologies, Inc. crystal for (a) short and (b) long TAC range data acquisition.

5.4 Amplifier shaping time dependence of CsI(Tl) pulse huight for crystals from Bicron Corporation, Horiba Crystal Products, Rexon Components, Inc., and Solon Technologies, Inc. Normalized to $12 \mu$ s pulse height of each crystal

5.5 Comparison of the modified Bollinger-Thumas (MBT) method and shaped square wave (SSW) method rise and decay time constant results for $\mathrm{CsI}(\mathrm{Tl})$

5.6 Comparison of the modified Bollinger-Thomas (MBT) method and shaped square wave (SSW) method charge contribution ratio results for $\operatorname{CsI}(\mathrm{Tl})$....

5.7 Comparison of the basic CsI(T1) pulse shape (rise and two primary decay components) from modified Bollinger-Thomas (MBT) and shaped square wave (SSW) results for Solon Technologies, Inc. crystal at $-60 \mathrm{C}$ 


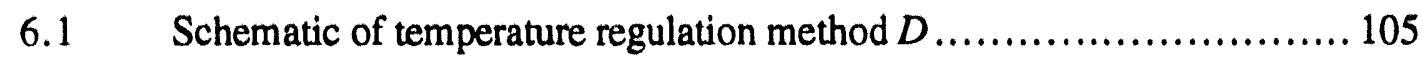

6.2 Current mode method results in temperature range of high thermoluminescence yield for temperature regulation method $A$. Data taken while changing from thermal equilibrium at $-50^{\circ} \mathrm{C}$ to thermal equilibrium at $-60^{\circ} \mathrm{C}$ for Solon Technologies, Inc. CsI(Tl) crystal ....... 106

6.3 Difference in current mode method scintillation yield results for temperature regulation methods $A$ and $C$ for Solon Technologies, Inc. CsI(Tl) crystal, normalized at $+20^{\circ} \mathrm{C}$........................................... 107

6.4 Difference in current mode method results observed when cooling and warming (temperature regulation methods $C$ and $D$, respectively)

Solon Technologies, Inc. CsI(Tl) crystal.

6.5 CSI(Tl) thermoluminescence "glow curves" for crystals from Solon Technologies, Inc., Bicron Corporation, Horiba Crystal Products, and Rexon Components, Inc., measured by the current mode method, normalized to room temperature scintillation yield of each crystal.

6.6 Comparison of 400 and $560 \mathrm{~nm}$ band thermoluminescence when quickly changing SSolon Technologies, Inc. CsI(Tl) crystal temperature from -60 to $-50^{\circ} \mathrm{C}$

A.1 Examples of centroid offeret ..................................... 126

A.2 Examples of (a) peak integration and (b) addition of Poisson noise ..... 127

A.3 Centroid results of moments calculations and least squares fitting for a peak area of 10,000 counts................................................... 128

A.4 FWHM results of moments calculations and least squares fitting for a peak area of 10,000 counts.

A.5 FWHM above which moments calculation uncertainties are within 5\% of the theoretical predictions ....................................... 130

B.1 CsI(TI)/photodiode geometry for DETECT ........................ 135

C.1 Photodiode calibration method schematic ........................... 138 


\section{LIST OF APPENDICES}

\section{Appendix}

A. Uncertainty of Centroid and Full-width at Half Maximum

Determinations.......................................................... 120

B. DETECT (Light Collection Efficiency) Input and Output Files........... 132

C. Photodiode Calibration Technique and Data ........................... 137

D. Instrumentation Impulse Response Function and CsI(TI) Rise and

Decay Component Results for Modified Bollinger-Thomas Method...... 139 


\section{CHAPTER I \\ INTRODUCTION}

Gamma-ray detectors with high efficiency, low power consumption, low maintenance, and small size and weight have long been nceded for many applications. While this ideal detector has not yet been achieved, the continuing development of solidstate photodiodes with large area and low noise has resulted in significant progress toward this realization. Silicon PIN photodiodes (PDs) have become one of the most commonly used photodetectors and thallium-activated cesium iodide, $\mathrm{CsI}(\mathrm{Tl})$, is one scintillating crystal that has been widely used with these PDs. Although CsI(TI) was recognized as a scintillating crystal that could be used for gamma-ray detection as early as 1950 by Hofstadter [HOF50], its use has been limited due to the poor match of its emission spectrum with the quantum efficiency of commercially-available photocathodes. However, the CsI(Tl) emission spectrum is very well matched with silicon PIN PDs, yielding a wavelength-averaged internal quantum efficiency of approximately $90 \%$ and an external quantum efficiency of about $70 \%$ (Geist, et al. discuss the difference between internal and external quantum efficiency [GEI82]). Fig. 1.1 shows sow the emission spectra of both $\mathrm{NaI}(\mathrm{Tl})$ and $\mathrm{CsI}(\mathrm{Tl})$ match the external quantum efficiencies of a S-11 photocathode and a typical PD. The original investigations of the CSI(Tl)/PD detector were performed in the 1960's [FAN64, KEI68, BAT69], but it has only been in the past decade that practical room temperature detectors have been available. $\mathrm{CsI}(\mathrm{Tl})$ is an efficient absorber of gamma rays, with a radiation length of $1.65 \mathrm{~cm}$ at $662 \mathrm{keV}$, a density of $4.51 \mathrm{~g} / \mathrm{cm}^{3}$, and an effective atomic number of 54 . CsI(Tl) has a refractive index of 1.8 in the visible spectrum. 


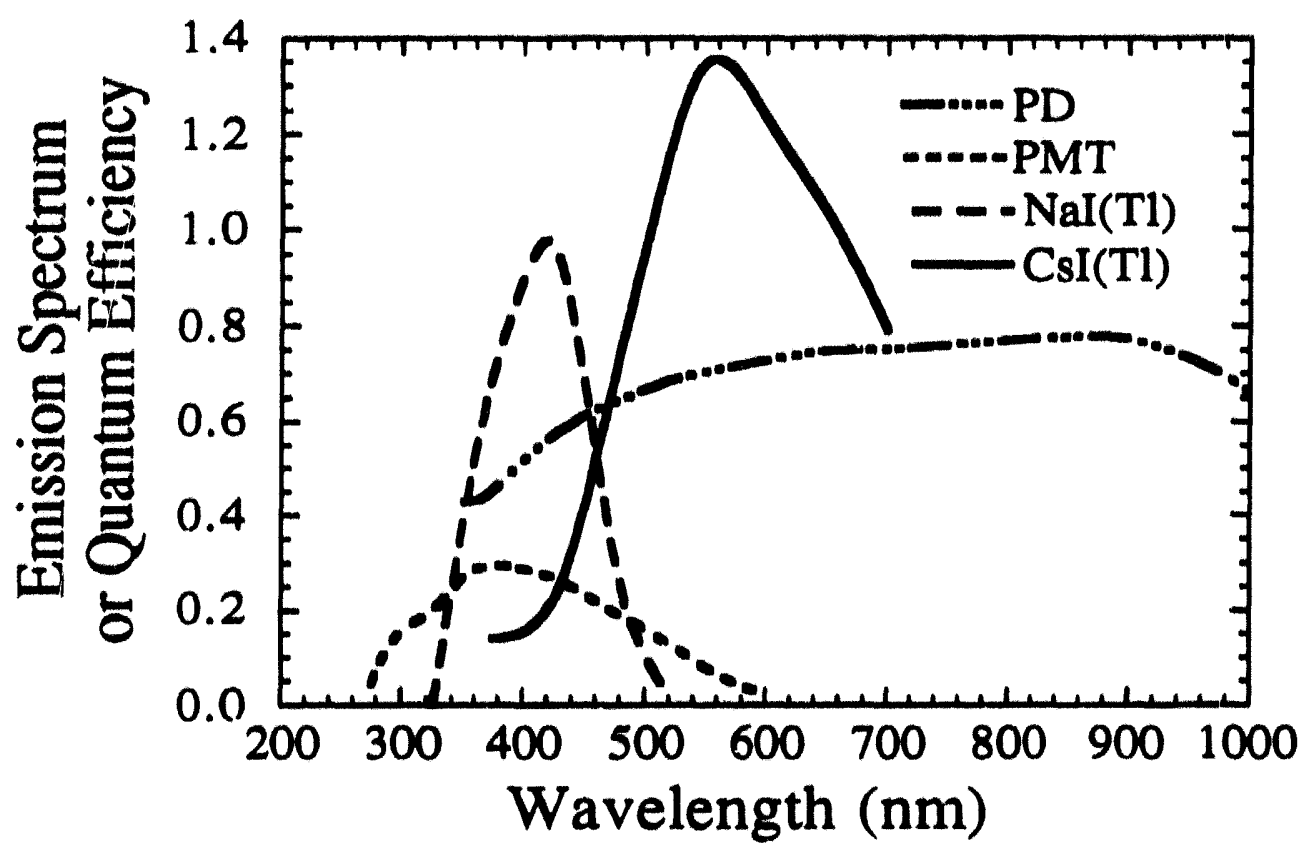

Figure 1.1: Comparison of the emission spectra of $\mathrm{NaI}(\mathrm{Tl})$ and $\mathrm{CsI}(\mathrm{Tl})$ and the quantum efficiencies of a S-11 photocathode (PMT) and a silicon PIN photodiode (PD). Emission spectra are normalized to $\mathrm{NaI}(\mathrm{TI})$ peak emission.

Due to the poor spectral match of the CsI(Tl) emission spectrum with typical photomultiplier tube sensitivities, the scintillation efficiency, and thus the absolute scintillation yield, of $\operatorname{CsI}(\mathrm{Tl})$ at room temperature was believed to be about $45 \%$ of that from $\mathrm{NaI}(\mathrm{Tl})$ [HEA79]. Since PDs have become available, more accurate measurements have revealed that $\mathrm{CsI}(\mathrm{TI})$ has the largest absolute scintillation yield at room temperature of known inorganic scintillating crystals [HOL88, SAK87, VAL93b].

The scintillation emissions of $\mathrm{CsI}(\mathrm{Tl})$ have also been reported to be dependent upon the excitation mechanism [ALA86, BEN89, HRE60, KRE87, MEI88, OWE59, ROB59, ROB61, STO58]. One of the CsI(Tl) scintillation decay time constants is observed to be dependent on the ionization density of particles being detected. Thus, by pulse-shape analysis the atomic number of the ionizing particle can be determined. CsI(Tl) has been used for particle identification in light ion physics applications, and has been proposed to be used in heavy ion physics applications by Benrachi, et al. [BEN89]. 
CsI(Tl) crystals coupled to a PD have been studied for use in many different applications. Kilgus, et al. [KIL90] evaluated the CsI(TI)/PD detector for use in low-level spectroscopy, while Grassmann, et al. [GRA85] and Bluchu, et al. [BLU85] have proposed and are building electromagnetic calorimeters for high-energy physics experiments and Viesti, et al. [VIE86] and Gong, et al. [GON88] propose intermediate energy (10-100 MeV) applications. The CsI(Tl)/PD detector has also been proposed to be used in current mode to monitor quasi-monochromatic photons generated by Compton hackscattering of laser light against relativistic electrons [OHG92] and to monitor the beam current of particle accelerators. For applications that involve imaging gamma rays, the CsI(TI)/PD detector has several favorable characteristics. CsI(TI) is a soft, ductile material that can be easily machined and is only slightly hygroscopic, while PDs are small, rugged, insensitive to magnetic fields, have low power consumption, and have good long term stability. Consequently, the CsI(TI)/PD detector is a likely candidate for many imaging applications. Dean, et al. [DEA87] are building a imaging camera based on Compton scattering with multiple CsI(TI)/PD detectors for gamma-ray astronomy, while others have proposed the use of CsI(Tl) in different medical imaging modalities, such as radiation oncology and $x$-ray computed tomography ( $x$-ray CT) [FAR82]. Jing, et al. [JIN92] have evaluated the use of $\mathrm{CsI}(\mathrm{Tl})$ with amorphous silicon photodiodes for hoth conventional and digital radiography. Additionally, we are interested in the imaging of gamma-ray fields in the nuclear reactor environment.

Even though the interest in CsI(Tl) is well-established for many applications, we have found that for many of the $\mathrm{CsI}(\mathrm{Tl})$ scintillation characteristics and their temperature dependence there is poor agreement among reported values and that some of the characteristics are incorrectly represented. Therefore, we have measured the temperature dependence of the gamma-ray excited scintillation characteristics of CsI(TI) and we attempt to explain our results with previously reported information regarding the solid state physics of the scintillation process in CsI(Tl) and other alkali halides. Section 1.1 is a compilation 


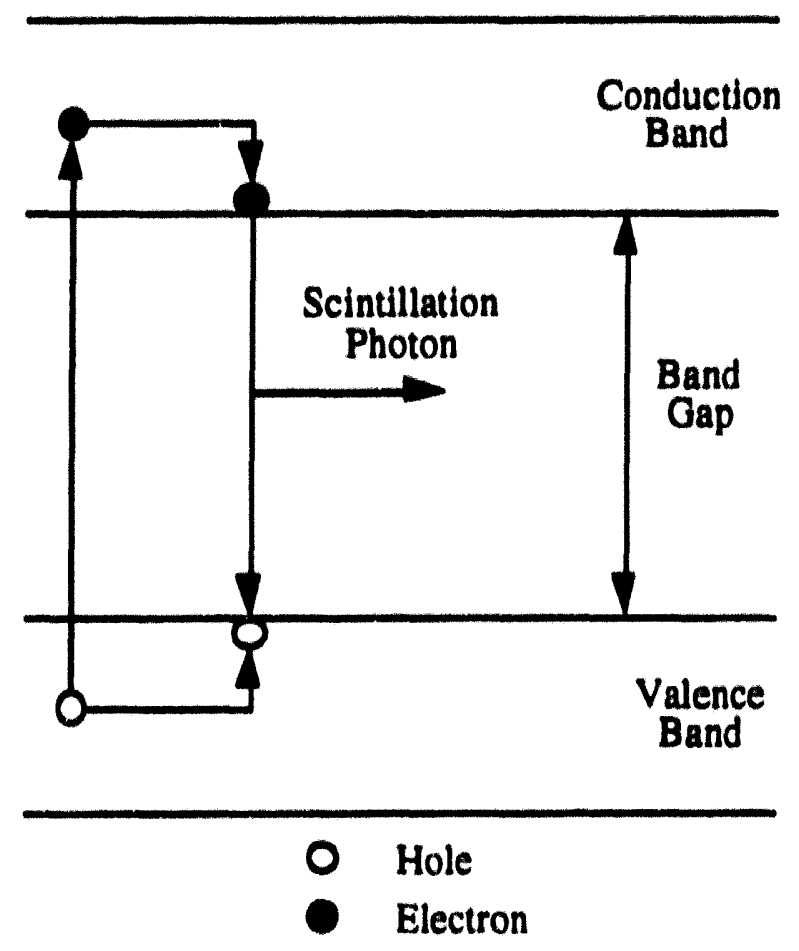

Figure 1.2: Schematic of scintillation process

of previously reported scintillation mechanisms in CsI(TI) and other alkali halides, while Section 1.2 describes which scintillation characteristics are of interest when evaluating CsI(Tl) as a gamma-ray detector and discusses the previously reported values of those characteristics.

\subsection{Scintillation Mechanisms}

Gamma rays interact in a detector via the photoelectric effect, Compton scattering, and pair production creating one or more "high" energy electrons (also known as delta rays). As these "high" energy electrons travel through the detector they transfer kinetic energy to the detector. For scintillating crystals, some of the deposited energy will raise electrons from the valence band across the band gap to the conduction band. As seen in Fig. 1.2, some of the conduction band electrons and the holes left in the valence band will recombine to emit scintillation photons (usually in the visible region of the electromagnetic 


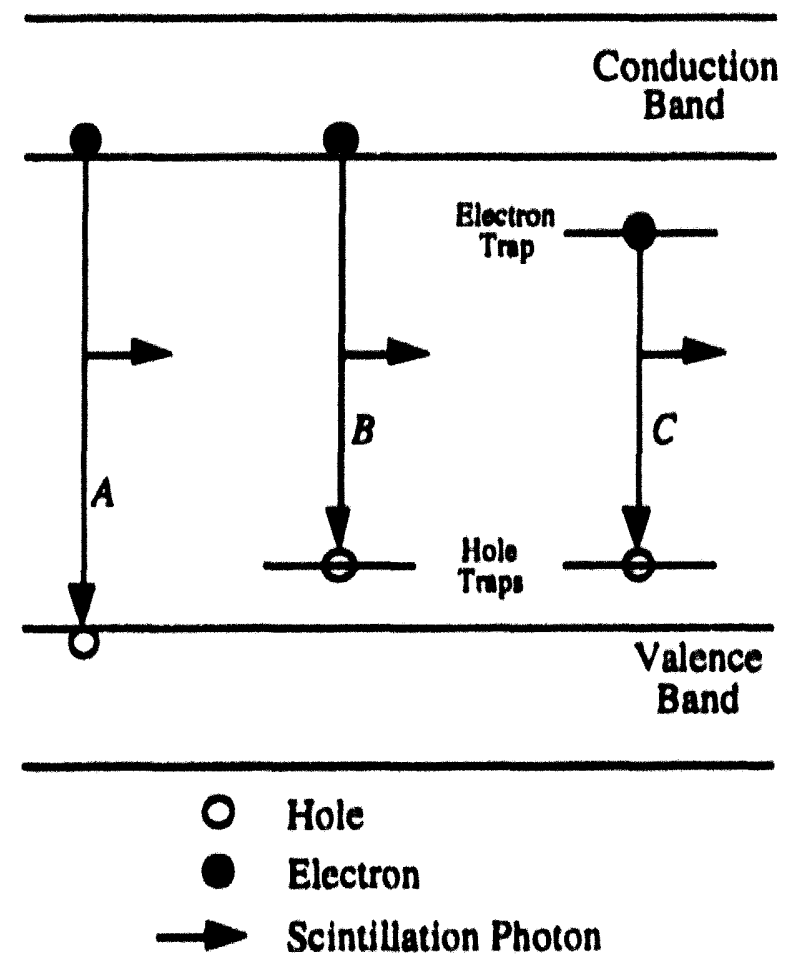

Figure 1.3: Three interband luminescence processes that are typically used to explain the emission of photons by scintillators.

spectrum). Recombination typically occurs after the electrons and holes move through the hands to luminescence centers.

Some kinetic energy from the "high" energy electron will not create conduction hand electrons, while some of the conduction band electrons will return to the valence band hy non-radiative processes, and some energy will be lost to phonons while the electron is changing energy levels in the conduction band. The scintillation efficiency is the energy emitted as scintillation photons divided by the energy deposited by the gamma ray and has heen used as a figure-of-merit for scintillators.

The emission of photons by scintillators has been described primarily by different types of interband radiative transitions of conduction band electrons to the valence band. Fig. 1.3 shows the three most predominant mechanisms that are responsible for these transitions. Edge luminescence (mechanism $A$ ) is caused by the annihilation of free excitons and is characterized by decay times on the order of picoseconds. Both 
mechanisms $B$ and $C$ are associated with localized electronic levels due to crystal defects, impurities, or activators. Mechanism $B$ represents the annihilation of a self-trapped exciton formed by a free valence band electron and a self-trapped hole. Mechanism $C$ is governed hy the thermally-activated migration of intrinsic or impurity centers and both $B$ and $C$ are characterized by decay times in the range of nanoseconds to micruseconds [DE192]. The following sections describe several such scintillation processes that have been reported to he prominent in activated alkali-halides and to contribute to the CsI(TI) scintillation mechanism.

\subsubsection{Thallium Luminescence Centers}

Small concentrations of thallium $(0.01$ to 0.1 mole $\%)$ are typically added as an activator to both pure Csl and NaI during the crystal growth process to improve the scintillation efficiency of these crystals. Consequently, the scintillation mechanism of both $\mathrm{CsI}(\mathrm{TI})$ and $\mathrm{NaI}(\mathrm{TI})$ is primarily governed by mechanism $C$ in Fig. 1.3. The role of thallium in alkali halide scintillation processes has heen studied by many different groups with somewhat inconsistent resulting conclusions [DIE72, DON68, FON68, GUT74, GWI63, KAU70, MOR59, SEI38, SHA69, VAN56, VAN64]. Thus, we will present some of the general properties that seem to be consistent throughout.

Shamovskii and Shushkanov [SHA69] performed crystallochemical analysis on CsI(TI) crystals to determine the crystalline structure and how the thallium activator embeds itself in the crystal structure. They reported that $\mathrm{Tl}+$ embedded itself with two different associations to the I- ions in the face-centered cubic CsI crystal; the $\mathrm{Tl}^{+}$was either surrounded by three or nine $\mathrm{I}^{-}$ions. These two different associations of $\mathrm{Tl}^{+}$with $\mathrm{I}^{-}$ions result in two different thallium luminescence centers [GUT74].

Dietrich and Murray [DIE72] and Kaufman, et al. [KAU70) agree that there are two different processes for the recombination of electrons $\left(c^{-}\right)$and holes $\left(h^{+}\right)$with the 
accompanying emission of a photon at $\mathrm{Tl}^{+}$sites. First, an electron could be initially trapped at the $\mathrm{Tl}^{+}$site, followed by recombination with a hole:

$$
\begin{aligned}
& \mathrm{e}^{-}+\mathrm{Tl}^{+} \rightarrow \mathrm{Tl}^{0} \\
& \mathrm{~h}^{+}+\mathrm{Tl}^{0} \rightarrow\left(\mathrm{Tl}^{+}\right)^{+} \rightarrow \mathrm{Tl}^{+} \text {+ photon. }
\end{aligned}
$$

The second process is the hole being trapped first followed by recombination with an electron:

$$
\begin{aligned}
& \mathrm{h}^{+}+\mathrm{Tl}^{+} \rightarrow \mathrm{Tl}^{++} \\
& \mathrm{e}^{-}+\mathrm{Tl}^{++} \rightarrow\left(\mathrm{Tl}^{+}\right)^{-} \rightarrow \mathrm{Tl}^{+}+\text {photon. }
\end{aligned}
$$

Consequently, electrons are trapped at $\mathrm{T}^{+}$ions as $\mathrm{T}^{\circ}$ centers while holes are trapped at $\mathrm{Tl}^{+}$ ions as $\mathrm{T}^{++}$centers. Both groups, as well as Donahue and Teegarden [DON68], Gutan, et al. [GUT74], and Gwin and Murray [OWI63], add that holes may be self-trapped at $V_{k}$ centers (discussed in Section 1.1.3) that are adjacent to a $\mathrm{Tl}+$ ion. The recombination of an electron with a $V_{k}$ center could follow either process.

Van Sciver [VAN56] reports that the transition of some of the excited T1+ states, $\left(\mathrm{TT}^{+}\right)^{*}$, to the ground state, $\mathrm{Tl}^{+}+$photon, are forbidden due to the band structure of $\mathrm{Tl}^{+}$for $\mathrm{Nal}(\mathrm{Tl})$. We assume that the excited $\mathrm{T}^{+}$states of $\mathrm{Cs}(\mathrm{Tl})$ will display similar behavior. At high temperatures, the trapped $\left(\mathrm{TI}^{+}\right)^{*}$ can be thermally activated to a state that does not have a forbidden transition to the ground state. However, at low temperatures the trapping of $\left.(\mathrm{T})^{+}\right)^{*}$ will result in stored energy that will be released as thermoluminescence when the crystal is heated.

\subsubsection{F Center Luminescence}

One other mechanism that has theen reported to trap electrons in alkali halides is the inrmation of F centers [JOH53, PIC66, SEI46]. F centers are formed in CsI(TI) when an electron is trapped in an I- vacancy, as seen in Fig. 1.4. Consequently, the formation of F centers is an intrinsic mechanism of the crystal, whereas the recombination of these centers with holes typically occurs at impurity or activator sites. Like electrons trapped at a TI+ 


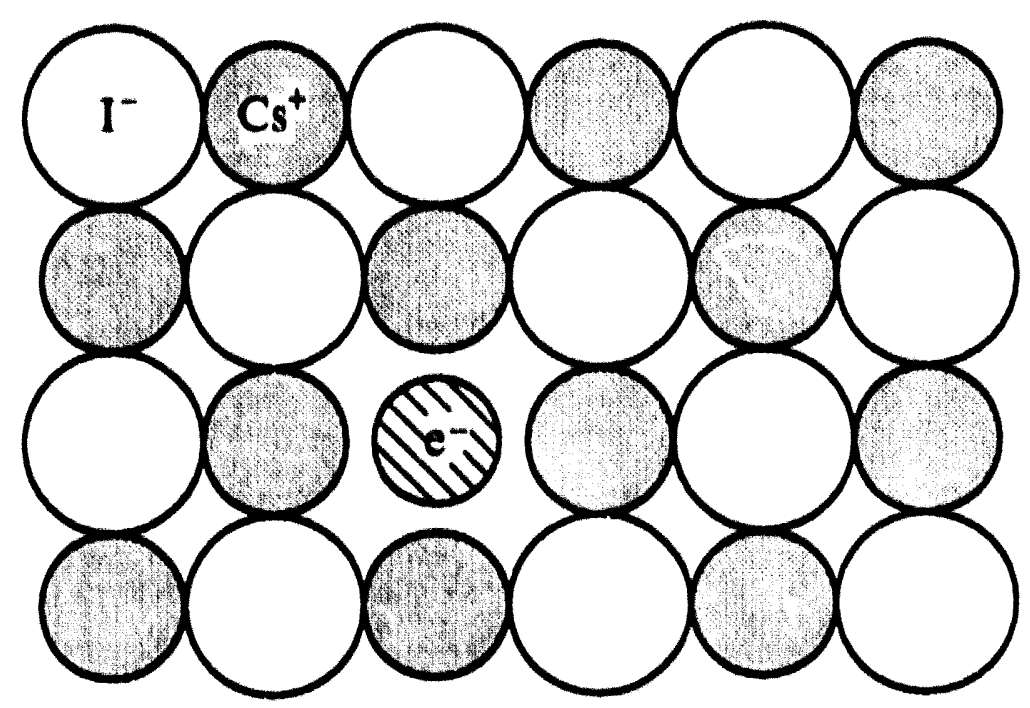

Figure 1.4: Diagram of F center in CsI(TI).

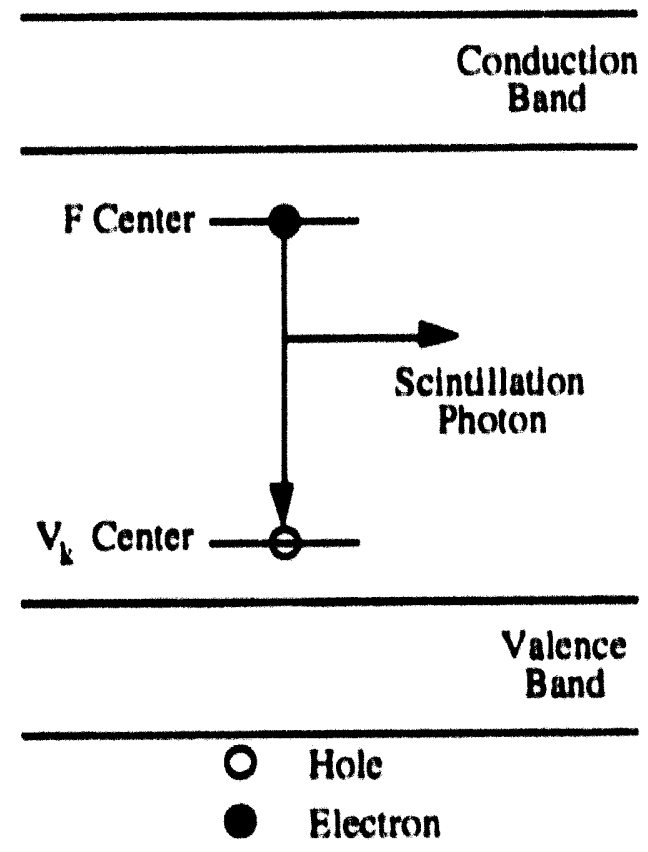

Figure 1.5: Schematic of luninescence process at an F center.

site, $F$ centers can recombine with holes with the resulting emission of a luminescence photons. Johnson and Williams [JOH53] report that F centers predominantly recombine with $\mathrm{V}_{k}$ centers that are self-trapped adjacent to a $\mathrm{Tl}^{+}$site for $\mathrm{KCl}(\mathrm{Tl})$, as seen in Fig. 1.5. and Pick [PIC66] reports that most alkali halides display similar F center characteristics. 
Thus, $\mathrm{F}$ centers in CsI(TI) probably recombine with $V_{k}$ centers in a similar fashion to $\mathrm{KCl}(\mathrm{Tl})$.

\subsection{3 $V_{k}$ centers}

As previously mentioned, holes may be self-trapped as $\mathrm{V}_{\mathrm{k}}$ centers adjacent to a $\mathrm{Tl}^{+}$ ion. $A V_{k}$ center for $\operatorname{CsI}(T I)$ is a pair of $1-$ ions that are coupled together to form a hole. $I_{2}$. when an electron is removed, as seen in Fig. 1.6. $V_{k}$ centers can be created anywhere in the crystal, but only contribute to thallium luminescence when self-trapped adjacent to a $\mathrm{Tl}+\mathrm{ion}$. Consequently, the $\mathrm{V}_{\mathrm{k}}$ centers must move through a crystal hy thermally-activated reorientation [DIE72, MUR75, SON70, VIT66]. As seen in Fig. 1.6, the reorientation can he either $90^{\circ}$ or $180^{\circ}$ to the coupled $\mathrm{I}_{2}^{-}$. When the third dimension of the face-centered cubic crystal is accounted for a $60^{\circ}$.reorientation will also be apparent. Although there are more possible 6$)^{\circ}$-reorientation sites, the activation energy is largest for this mode, while a $V_{k}$ center will not move via $180^{\circ}$-reorientation on average because it will have a $50 \%$ chance of going one of two directions for each reorientation. Consequently, $90^{\circ}$. reorientation is the most likely means of transporting $\mathrm{V}_{\mathrm{k}}$ centers to $\mathrm{Tl} \mathrm{l}^{+}$sites in CsI(Tl). Once at the $\mathrm{Tl}^{+}$sites, the self-trapped hole can recombine with a free electron or an electron that is either trapped at a $\mathrm{Tl}^{+}$center or an $\mathrm{F}$ center to create a scintillation photon. Donahue and Teegarden [DON68] and Gutan, et al. [GUT74] both conclude that the recombination process for $\mathrm{V}_{\mathrm{k}}$ centers that are adjacent to $\mathrm{T} 1^{+}$ions follows

$$
\begin{aligned}
& \mathrm{I}_{2}^{-}+\mathrm{Tl}^{+} \rightarrow \mathrm{I}_{2}^{-} \mathrm{Tl}^{+} \\
& \mathrm{e}^{-}+\mathrm{I}_{2}^{-} \mathrm{Tl}^{+} \rightarrow\left(\mathrm{I}_{2}^{-}\right)^{+} \mathrm{Tl}^{+} \rightarrow 2 \mathrm{I}^{-}+\mathrm{Tl}^{+}+\text {photon. }
\end{aligned}
$$

where the $\mathrm{e}^{-}$can be a previously trapped or free electron. This recombination process is sinilar to the processes described in 1.1.1, except that the excited ion in this process is the $V_{k}$ center instead of the $T+1$ ion. 


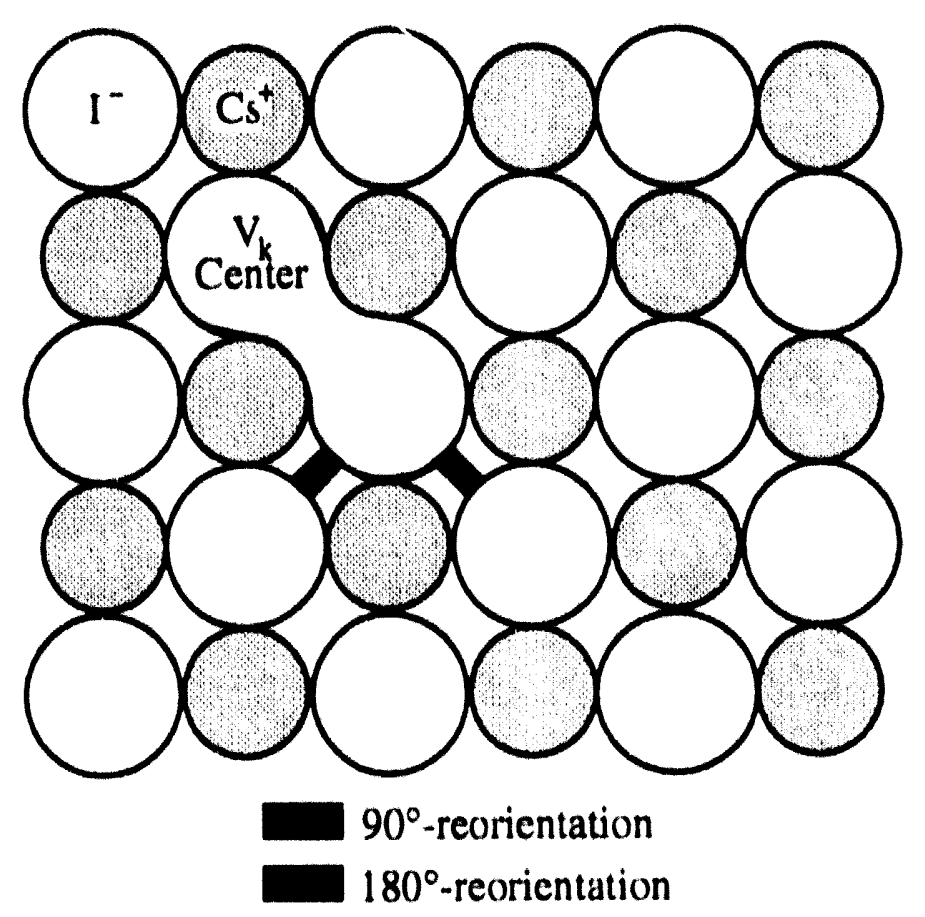

Figure 1.6: Diagram of $\mathrm{V}_{k}$ center in CsI(Tl) with possible directions for $90^{\circ}$ and $180^{\circ}$. reorientation.

\subsubsection{Intraband Luminescence}

Although the bulk of scintillation photons are accounted for by interband transitions, two different intraband transitions have been reported to account for luminescence in scintillators. Cross-luminescence has been observed to account for the fast component of barium fluoride, $\mathrm{BaF}_{2}$, and some other crystals with similar band structures to $\mathrm{BaF}_{2}$ [LAV83], while alkali halides and some other ionic crystals have teen reported to umit intraband luminescence [DE192]. Intraband luminescence can originate in either the valence or conduction band, while cross luminescence is only observed to originate in the valence band, as seen in Fig. 1.7. For valence hand origination, intraband luminescence is the result of a "high" energy electron (created hy a gamma ray interaction) transferring its kinetic energy to a deep valence band electron leaving a deep hole in the upper valence band or an inner valence band. The deep hole can then be filled by a higher energy valence band 

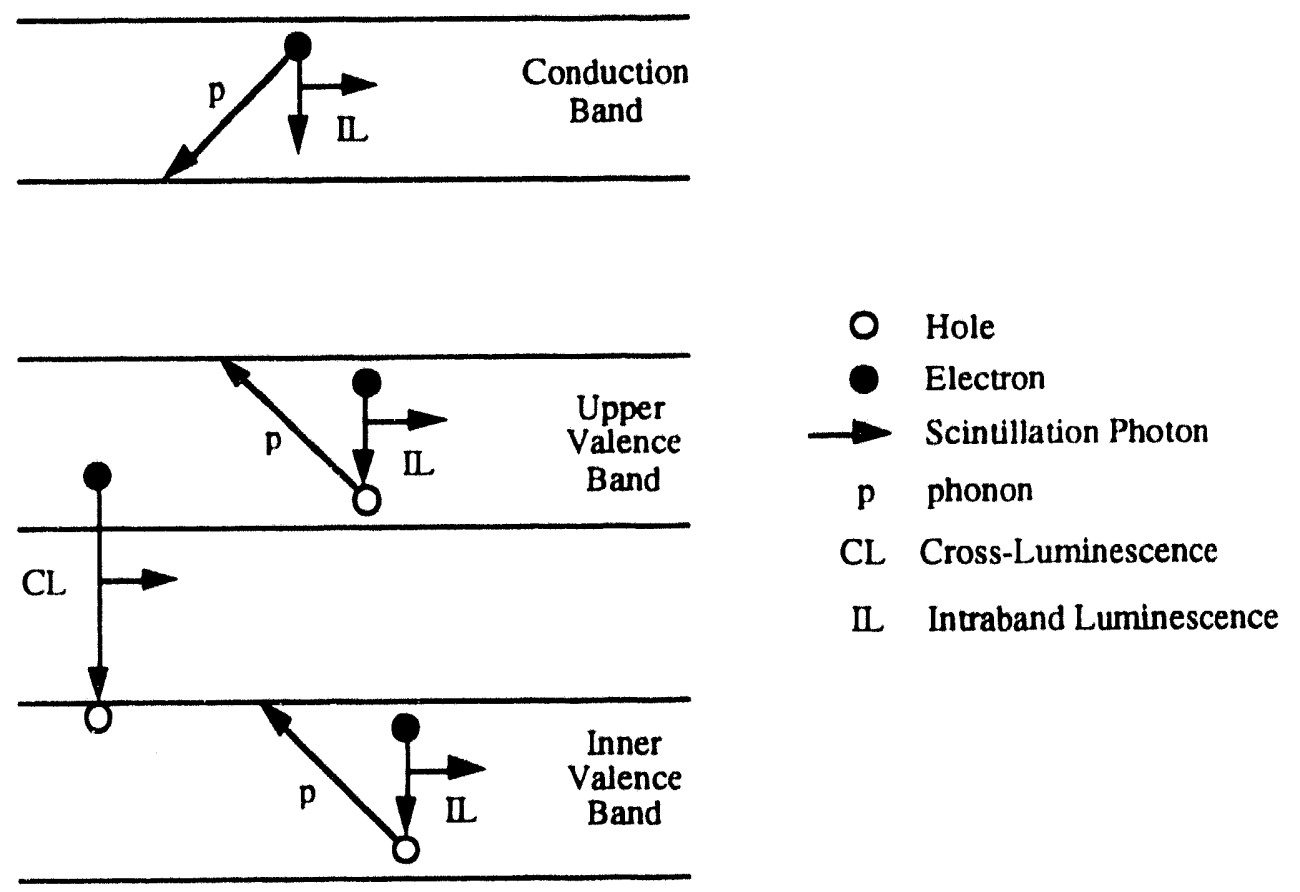

Figure 1.7: Schematic of intraband and cross-luminescence along with competing phonon process for intraband luminescence.

electron accompanied by the emission of an intraband luminescence photon or relax to the top of its valence band via phonon emission. This scintillation mechanism is characterized by decay times on the order of picoseconds because the competing phonon process occurs in that time frame. Cross luminescence occurs when a hole in an inner valence band relaxes to the top of that band and is subsequently filled by an upper valence band electron that is accompanied by the emission of a photon and is characterized by decay times on the order of a nanosecond. For the conduction band origination of intraband luminescence, a valence band electron is excited into the conduction band and moves toward the bottom of the band by either intraband luminescence or phonon emission. Conduction band intraband luminescence is also characterized by decay times on the order of picoseconds. Intraband luminescence is one of the fastest known scintillation processes, but typically has a scintillation efficiency of $10^{-4}$ to $10^{-5}$ for alkali halides as compared to an overall scintillation efficiency of about 0.14 for both $\mathrm{NaI}(\mathrm{Tl})$ and $\mathrm{CsI}(\mathrm{Tl})$. Since intraband luminescence is governed by the band structure of the host crystal, both the emission 
spectrum and scintillation efficiency will be independent of impurities and activators in the crystal. Additionally, the band structure does not change significantly with temperature, and thus the intraband luminescence emission spectrum and scintillation efficiency will be approximately independent of temperature [DEI92].

\subsection{Scintillation Characteristics that Affect Detector Performance}

To aid investigators in assessing the use of $\mathrm{CsI}(\mathrm{Tl})$ for a particular application we have measured the gamma-ray excited scintillation characteristics that are critical to detector performance. In addition to measuring the scintillation characteristics of interest at room temperature, we have also measured the temperature dependence of these characteristics. The temperature dependence of $\mathrm{CsI}(\mathrm{Tl})$ is of interest for two reasons. First, the thermally excited dark current present in silicon PIN PDs at room temperature limits the achievable energy resolution and suggests that low temperature operation might result in better energy resolution since the dark current will decrease exponentially with inverse temperature. Second, some applications require ambient temperatures other than typical room temperature. Consequently, the temperature dependence of the CsI(Tl) scintillation characteristics has been studied in the temperature range of -100 to $+50^{\circ} \mathrm{C}$.

The solid state physics of the scintillation mechanism is of interest primarily to aide in the search for better scintillators. However, the manifestations of the scintillation mechanism comprise two important characteristics, the emission spectrum and the temporal hehavior of the gamma-ray excited luminescence. The emission spectrum is the distribution of scintillation photons as a function of wavelength. As previously mentioned, the emission spectrum can dictate the photodetector that will best convert scintillation photons to charge carriers, quantum efficiency, that can be processed by electronics. The emission spectrum averaged over the photodetector quantum efficiency along with the scintillation efficiency determine the number of charge carriers created by a scintillation event, $\mathrm{N}_{\mathrm{cc}}$. Since the statistical fluctuations of $\mathrm{N}_{\mathrm{cc}}$ will be the limiting factor of detector 
energy resolution when a detector is operated in pulse mode, it is advantageous to know both the emission spectrum and scintillation efficiency of a scintillator. Furthermore, the scintillation efficiency can be determined by measuring the emission spectrum and the absolute scintillation yield.

For gamma-ray excitation at room temperature the emission spectrum of CsI(TI) has heen reported to have up to four emission bands, the most prevalent of which peaks at about $560 \mathrm{~nm}$ [CRA74, GRA84, GRA85, GUT74, GWI63, KRE87, LAG61, MAS66, SCH90, TOW68]. Measuring the absolute shape of the emission spectrum requires correcting recorded data for wavelength-dependent variations in monochromator efficiency and photocathode quantum efficiency. It is not stated in most cases whether the previously reported emission spectra have been corrected for such variations. We have corrected our data accordingly to yield an absolute emission spectrum shape.

The absolute scintillation yield is a dominant factor in determining the energy resolution of a scintillation detector because the yield governs pulse amplitude, as well as the statistical fluctuations in the number of charge carriers created and the scintillation efficiency. Electronic noise and the uniformity of the light collection efficiency across the volume of the scintillator are also energy resolution factors that must be considered, but the absolute scintillation yield is typically the limiting contribution to energy resolution. Since $\mathrm{CsI}(\mathrm{Tl})$ has the largest known scintillation yield, it has the potential for producing the best energy resolution of any scintillating crystal. Holl, et al. [HOL88] have reported that the CsI(Tl) absolute scintillation yield is 51,800 photons $/ \mathrm{MeV}$ at room temperature, but did not account for ballistic deficit due to the long decay time of $\mathrm{CsI}(\mathrm{Tl})$. We have reported a room temperature absolute scintillation yield of 64,800 photons $/ \mathrm{MeV}$ using a method that accounts for ballistic deficit [VAL93b]. The temperature dependence of the CsI(Tl) absolute scintillation yield is also of importance because some applications require an operating temperature other than room temperature. Previous relative measurements of the scintillation yield temperature dependence [BLU86, GRA85, KOB89] give an indication of 
this behavior, but do not account for long scintillation decay time constants and the variation of these decay times with temperature. Kobayashi, et al. [KOB89] have demonstrated that the temperature dependence of the CsI(TI) scintillation yield for gamma rays does not vary with incident gamma-ray energy. Thus, if the temperature dependence of the absolute scintillation yield is measured for any single gamma-ray energy, the result should apply to all gamma-ray energies. Additionally, the scintillation yield of CsI(Tl) is known to be dependent on thallium concentration, unlike the decay times [SCH90]. Consequently, crystal-to-crystal variations of absolute scintillation yield are expected. This thallium concentration dependence may also manifest itself in differences in the temperature dependence of the scintillation yield.

When a detector is operated in pulse mode, the temporal behavior of the scintillation emissions determine the timing capabilities, high count rate limitations, and in some cases the ability to utilize the charge carriers created by the detector. The temporal behavior is characterized by rise and decay times that determine when scintillation photons are emitted with respect to the gamma-ray interaction. Many scintillator applications require using timing signals for coincidence measurements. The best timing signals are produced by scintillators that have high light yield with fast rise and decay times. The number of gamma-ray excited decay modes, their decay times, and their temperature dependence for CsI(TI) have been the subject of some controversy; both one and two primary decay modes have been reported [GRA84, GRA85, JON60, MAS66, MOR59, PLI59, SAS61, SCH90, STO58, VAL93a]. Also, the presence of a significant exponential rise time has been recognized, but it has only been quantified by Grassmann, et al. [GRA85] and Valentine, et al. [VAL93a]. Table 1.1 shows the previously reported values of the rise time constant, $\tau_{\text {rise }}$, and the two primary decay time constants of $\operatorname{CsI}(\mathrm{Tl}), \tau_{1}$ and $\tau_{2}$, at room temperature. The confusion about the number of decay modes might be due to the length and relatively small initial intensity of the longer component. However, the longer component is responsible for about $40 \%$ of the scintillation yield and leads to significant ballistic deficit 
Table 1.1: Published values of primary decay time constants at room temperature.

\begin{tabular}{|l|c|c|c|}
\hline Author(s) & $\begin{array}{c}\tau_{\text {rise }} \\
(\mathrm{ns})\end{array}$ & $\begin{array}{c}\tau_{1} \\
(\mathrm{~ns})\end{array}$ & $\begin{array}{c}\tau_{2} \\
(\mu \mathrm{s})\end{array}$ \\
\hline Benrachi, et al. [BEN89] & - & 700 & 4.0 \\
Crannell, et al. [CRA74] & - & 800 & 1.6 \\
Grabmaier [GRA84] & - & 980 & - \\
Grassmann, et al. [GRA85] & 40 & 850 & - \\
Masunaga, et al. [MAS66] & - & 800 & 1.2 \\
Morgenshtern [MOR59] & - & 550 & 0.7 \\
Pliavin [PLI59] & - & 800 & 1.6 \\
Sastry and Thosar [SAS61] & - & 900 & 10 \\
Schotanus, et al. [SCH90] & - & $6(0)$ & 3.4 \\
Storey, et al. [STO58] & - & 700 & - \\
Valentine, et al. [VAL93a] & 19.6 & 679 & 3.34 \\
\hline
\end{tabular}

when $\operatorname{CsI}(\mathrm{Tl})$ is used with commercially-available linear amplifiers. The long decay component thus has a significant effect on pulse processing and the observed scintillation characteristics of $\mathrm{Csl}(\mathrm{Tl})$, e.g. energy resolution and light yield. Therefore, variations in the long decay time with temperature are of significant interest. The decay times of CsI(TI) have heen reported to be independent of thallium concentration for gamma-ray excitation [SCH90]. This thallium concentration independence 'ould result in small variation in decay times from crystal to crystal, independent of crystal urigin.

For current mode operation, as proposed for CsI(Tl) in high energy physics applications, the emission spectrum and the scintillation yield will again determine $\mathrm{N}_{\mathrm{cc}}$. However, if a scintillator emits thermoluminescent light in the temperature range of operation, current mode and some pulse mode results may be erroneous. CsI(Tl) is known 10 be thermoluminescent in the temperature range of -100 to $+50^{\circ} \mathrm{C}$ [GRA84, GUT74]. Therefore, we have investigated the temperature ranges over which thermoluminescence occurs and whether these ranges vary from crystal to crystal.

CsI(Tl) crystals from several vendors were investigated to assess the differences in scintillation characteristics. Cubic crystals of $8 \mathrm{~mm}$ on a side were procured from Bicron Corporation, Horiba Crystal Products, Rexon Components, Inc., and Solon Technologies, 
Inc. The STI crystal was chosen at random to be used for the bulk of the measurements. To assess the performance of $\operatorname{CsI}(T 1)$ as a gamma-ray detector, we have measured the emission spectrum (Chapter III), the absolute scintillation yield (Chapter IV), the rise and decay components (Chapter V), and the thermoluminescence emissions (Chapter VI). 


\section{CHAPTER II}

\section{THEORY}

\subsection{CaI(TI) Seintillation Pulses}

To determine the absolute scintillation yield, the scintillation process and all of the subsequent mechanisms for transforming the scintillation light to a usable electronic pulse must be considered. For the present discussion, only the mechanisms that lead to production of charge carriers in the photodetector will be considered. The processing of the pulse created by these charge carriers will be discussed in Section 2.2. The total number of charge carriers (electron-hole (e-h) pairs in a photodiode (PD) and photoelectrons in a photomultiplier tube (PMT)) created by a full-energy gamma-ray, $E_{\gamma}$ event at $1=0, N_{\infty}\left(E_{\gamma} T\right)$, can be written as

$$
N_{c c}\left(E_{\gamma}, T\right)=\int_{0}^{\infty} \int_{0}^{\infty} N\left(E_{\gamma}, \lambda, T, t\right) \eta(\lambda, T, t) Q(\lambda, T, t) d \lambda d t,
$$

where $N, \eta$, and $Q$ are the number of scintillation photons produced, light collection efficiency, and photodetector quantum efficiency, respectively, expressed as functions of wavelength $\lambda$, temperature $T$, and time $t$.

Consistent with earlier observations, we shall assume that the luminescent states of CsI(TI) are depopulated by exponential processes and are populated either exponentially, instantaneously, or by a combination of both. Using these ussumptions, the total number of scintillation photons produced at time $t$ can be expressed as

$$
N\left(E_{\gamma}, \lambda, T, t\right)=E_{\gamma} \sum_{j} \frac{a_{j}(T)}{\tau_{j}(T)} e^{-t / \tau_{j}(T)} \varepsilon_{j}(\lambda, T),
$$


where $a_{j}, \tau_{j}$ and $\varepsilon_{j}$ are the number of photons produced per unit energy deposited (integrated luminescence), the rise or decay time constant, and the emission spectrum of the jth luminescence mode. For all luminescence processes, the characteristic time is a decay time constant and the integrated luminescence takes on a positive value, $a_{\text {decay }}>0$. The exponential population of a luminescent state manifests itself as a separate luminescence mode in Eqn. (2.2). In this representation, the rise component behaves as a negative integrated luminescence $\left(a_{\text {rise }}<0\right)$, the characteristic time is a rise time constant, and the total integrated luminescence for a process with an exponential population feeding an exponential depopulation is $a_{\text {rieo }}+a_{\text {docay }}$. A negative integrated luminescence implies that the rise component reduces the total integrated luminescence from what it would be if the population of the luminescent state were instantaneous. If a luminescent state is populated only by a single exponential process then the absolute value of the initial intensities are equal, $I_{\text {decay }}=-I_{\text {rise }}$. Although it is well known that the emission spectrum is dependent on the luminescence mode for some scintillators such as $\mathrm{BaF}_{2}$, the emission spectrum of CsI(TI) has heen assumed to be independent of the luminescence mode and thus independent of time $\left(\varepsilon_{j}(\lambda, T)=\varepsilon(\lambda, T)\right)$ [VAL93a]. The assumption of emission spectrum temporal independence will be discussed further in Section 3.3. Consequently, by defining the temporal luminescence intensity $I(T, t)$ as the sum of the exponential components:

$$
I(T, I)=\sum_{j} \frac{a_{j}(T)}{\tau_{j}(T)} e^{-1 / \tau_{j}(T)}=\sum_{j} I_{j}(T) e^{-1 / \tau_{j}(T)} .
$$

where $I_{j}$ is the initial intensity per unit energy deposited of the $j^{\text {th }}$ exponential component. and Eqn. (2.2) can be re-written

$$
N\left(E_{\gamma}, \lambda, T, t\right)=E_{\gamma} I(T, \imath) \varepsilon(\lambda, T) .
$$

When the emission spectrum is normalized such that

$$
\int_{0}^{\infty} \varepsilon(\lambda, T) d \lambda=1,0,
$$

the integration of $I(T, t)$ over time results in the absolute scintillation yield 


$$
\int_{0}^{D} I(T, t) d t=\sum_{j} a_{j}(T)=N_{A b s}(T)
$$

\subsubsection{Light Collection Emcioncy, $\eta(\lambda, T, t)$}

The light collection efficiency, $\eta$, has conventionally been defined as the average probability of a scintllation photon of wavelength $\lambda$ being incident on the photodetector averaged over all possible positions of a scintillation event and directions of the emitted scintillation light. For a highly reflective system with good optical coupling between the scintillating crystal and the photodetector (conditions necessary to achieve optimal energy resolution for a scintillation detector), a single photon could be incident on the photodetector more than once. Thus, the possibility of a light collection efficiency greater than unity exists with this conventional definition and $\eta$ is no longer a legitimate probability. This $\eta$ is also impossible to measure experimentally because an incident photon can only be counted if it creates a charge carrier. Consequently, $\eta$ has been defined in this work as the average probability of a scintillation photon of wavelength $\lambda$ being absorbed in or transmitted through the photodetector. For the 30()$\mu \mathrm{m}$ thick silicon PIN photodiode used for determining absolute scintillation yield, it is reasonable to assume that no scintillation photons are transmitted through the photodetector. The definition of $\eta$ for a PD thus becomes the average probability of a scintillation photon of wavelength $\lambda$ being absorbed in the photodetector. $\eta$ has been assumed to be independent of time, temperature, and, as justified in Chapter V, scintillation photon wavelength. Thus we can treat the light collection efficiency as a constant, $\eta(\lambda, T, t)=\eta$.

\subsubsection{Quantum Emciency, $Q(\lambda, T, t)$}

Photodetector quantum efficiency is conventionally defined as the probability that a scintillation photon incident on the photodetector creates a charge carrier, i.e., an e-h pair 
for a photodiode. This definition can be more accurately described as the extemal quantum efficiency [GE182], $Q_{\text {exl }}$ and is inconsistent with our definition of $\eta$. Thus, we shall define $Q$ as the average probability that a scintillation photon which is absorbed in the photodiode creates an e-h pair. This definition of $Q$ is consistent with that of internal quantum efficiency and is related to $Q_{a x i}$ by

$$
Q=\frac{Q_{\text {eal }}}{1-p} \text {. }
$$

where $\rho$ is the reflectance of the photodiode [OEI82]. Although $Q_{\text {axl }}$ and $\rho$ are dependent both on the angle of incidence, $\theta$, and the wavelength, $\lambda$, of the acintillation photon, Oeist, et al. [GE182] have confirmed that $Q$ is independent of the incident angle. Therefore if $Q_{\text {oxt }}$ and $\rho$ are known at a particular incident angle, $\theta_{0}$, then $Q$ can be determined for all incident angles:

$$
Q(\lambda)=\frac{Q_{0 x 1}\left(\lambda, \theta_{0}\right)}{1-\rho\left(\lambda, \theta_{0}\right)}
$$

The incident angle independence of $Q$ lends further justification for our definitions of $\eta$ and Q. since the conventional definitions would require the angular distribution of the incident scintillation photons to determine $Q_{\text {axl. }}$. Our definitions are more rigorous than the conventional ones because all optical properties ure contained in $\eta$ and only semiconductor properties are contained in $Q$. Similarly, our definitions divide $\eta$ and $Q$ into measurable quantities, whereas measuring the number of photons incident on a surface is impossible unless all incident photons are detected. The internal quantum efficiency, referred to as quantum efficiency henceforth, is assumed to be independent of time, $Q(\lambda, T, 1)=Q(\lambda, T)$.

\subsubsection{Absolute Scintillation Yleld, $\mathrm{N}_{\mathrm{Ab},}(\mathrm{T})$}

Substituting Eqn. (2.4) into Eqn. (2.1) and using the results of Eqns. (2.5) and (2.6), the number of charge carriers created is

$$
N_{c c}\left(E_{y}, T\right)=E_{y} \eta N_{A b s}(T) \int_{0}^{\infty} e(\lambda, T) Q(\lambda, T) d \lambda .
$$


where it has been assumed that quantum efficiency is time independent and light collection efficiency is constant. The wavelength-averaged quantum efficiency, defined as

$$
\bar{Q}(T)=\int_{0}^{\infty} \varepsilon(\lambda, T) Q(\lambda, T) d \lambda
$$

is the wavelength-averaged probability of an absorbed scintillation photon creating an e.h pair in the PD at temperature T. Substituting Eqn. (2.10) into Eqn. (2.9) and rearranging resules in

$$
N_{A b_{1}}(T)=\frac{N_{o c}\left(E_{y}, T\right)}{E_{y} \eta Q(T)}
$$

Thus, by determining $N_{c e}\left(E_{r} T\right), \eta$, and $\bar{Q}$ and knowing $E_{\gamma}$ the temperature-dependent absolute scintillation yield can be determined.

\subsection{Charge Callbration Methods}

The temporal luminescence intensity of CsI(TI) scintillation light at room temperature is best represented by two decay components $\left(\left(a_{1}, \tau_{1}\right)\right.$ and $\left(a_{2}, \tau_{2}\right)$ where $\left.\tau_{1}<\tau_{2}\right)$, and a rise component ( $a_{\text {rise. }} \tau_{\text {rise }}$ ) that is associated with the shorter decay component $\left(I_{1}=-I_{\text {riteo }}\right)$ :

$$
\begin{aligned}
& I(1)=\frac{a_{1}}{\tau_{1}} e^{-1 / \tau_{1}}+\frac{a_{2}}{\tau_{2}} e^{-1 / \tau_{2}}+\frac{a_{\text {rine }}}{\tau_{\text {rive }}} e^{-1 / \tau_{\text {rim }}} . \\
& =1_{1} e^{-1 / \tau_{1}}+1_{2} e^{-1 / \tau_{2}}+1_{\text {nmo }} e^{-1 / \tau_{\text {mon }}} \text {. } \\
& =I_{1}\left(e^{-1 / \tau_{1}}-e^{-1 / \tau_{\text {rue }}}\right)+I_{2} e^{-1 / \tau_{2}} \text {. }
\end{aligned}
$$

As seen in Table 1.1 the longer decay time constant, $\tau_{2}$, is expected to be on the order of microseconds, while $\tau_{1}$ and $\tau_{\text {rise }}$ are expected to be on the order of hundreds and tens of nanoseconds, respectively.

After the scintillation light is converted to e-h pairs in the PD, the pulse will continue to have the same basic shape, since the PD rise time (a few nasoseconds) is short compared to the rise and decay time constants. The electron current pulse produced by a scintllation ovent is 


$$
I(t)=e E_{\gamma} \eta \bar{Q}\left[I_{1}\left(e^{-1 / t_{1}}-e^{-1 / t_{r} / t}\right)+I_{2} e^{-1 / t_{2}}\right] .
$$

where $\mathrm{e}$ is the electronic charge, and the corresponding total charge of the pulse is

$$
q_{C a 1}=\int_{0}^{\infty} r(t) d t=c E_{Y} \eta \bar{Q}\left[1_{1} \tau_{1}-I_{1} \tau_{\text {rim }}+I_{2} \tau_{2}\right] \text {. }
$$

Defining $q_{1}=c E_{\gamma} \eta \bar{Q} I_{1} \tau_{1}$ and $q_{2}=e E_{\gamma} \eta \bar{Q} l_{2} \tau_{2}$. Eqn. (2.14) simplifies to

$$
q_{\text {cal }}=q_{1}-q_{1} \frac{\tau_{\text {sine }}}{\tau_{1}}+q_{2} \text {. }
$$

and Eqn. (2.13) becomes

$$
i(t)=\frac{q_{1}}{\tau_{1}}\left(e^{-1 / \tau_{1}}-e^{-1 / t_{\text {rim }}}\right)+\frac{y_{2}}{\tau_{2}} e^{-1 / t_{2}}
$$

When this current pulse is introduced into charge-sensitive preamplifier having $\tau_{p}=R_{F} C_{F}$ as the time constant of the charge integrator, the current pulse is integrated over the feedhack capacitor, $C_{\mu}$, and yields a voltage pulse with the following time dependence when $\tau_{p} \gg \tau_{1}, \tau_{2}, \tau_{\text {rises }}$ :

$$
V_{\text {Preump }}(t)=\frac{q_{C_{1}} l}{C_{p}} e^{-1 / \tau_{p}} \cdot \frac{q_{L}}{C_{p}}\left(e^{-1 / \tau_{1}}-\frac{\tau_{\text {rine }}}{\tau_{1}} e^{-1 / \tau_{\text {riso }}}\right) e^{-1 / \tau_{1}}-\frac{q_{z_{2}}}{C_{p}} e^{-1 / \tau_{2}}
$$

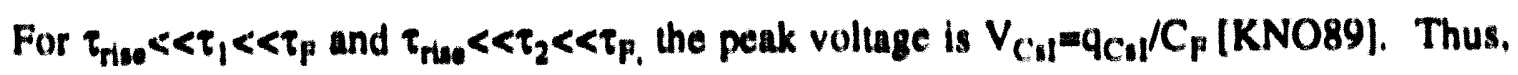
the preamplifier peak voltage is directly proportional to the total charge induced by the CsI(TI) scintillation. Figure 2.1 demonstrates the derived pulse shapes for a CsI(TI) scintillation pulse. This model represents an ideal preamplifier behavior, but the actual components function consistently with this model.

When the preamplifier output pulses are subsequently shaped by a linear amplifter. it is necessary to have an amplifier shaping time larger than four times the longest decay time to preserve $99 \%$ of the preamplifier output pulse amplitude. Thus, some hallistic deficit is unavoidably created when $\mathrm{Cs}(\mathrm{TT})$ scintillation pulses are shaped by commerciallyavailahle linear amplifiers (shaping time $s 12 \mu \mathrm{s}$ ) due to the large value of $\tau_{2}$. The amount of ballistic deficit will be smaller with increasing shaping time. thus the amplitude of the shaped pulse will increase with increasing shaping times. The following two sections describe why the two methods that have been traditionally used for calibrating amplifier 


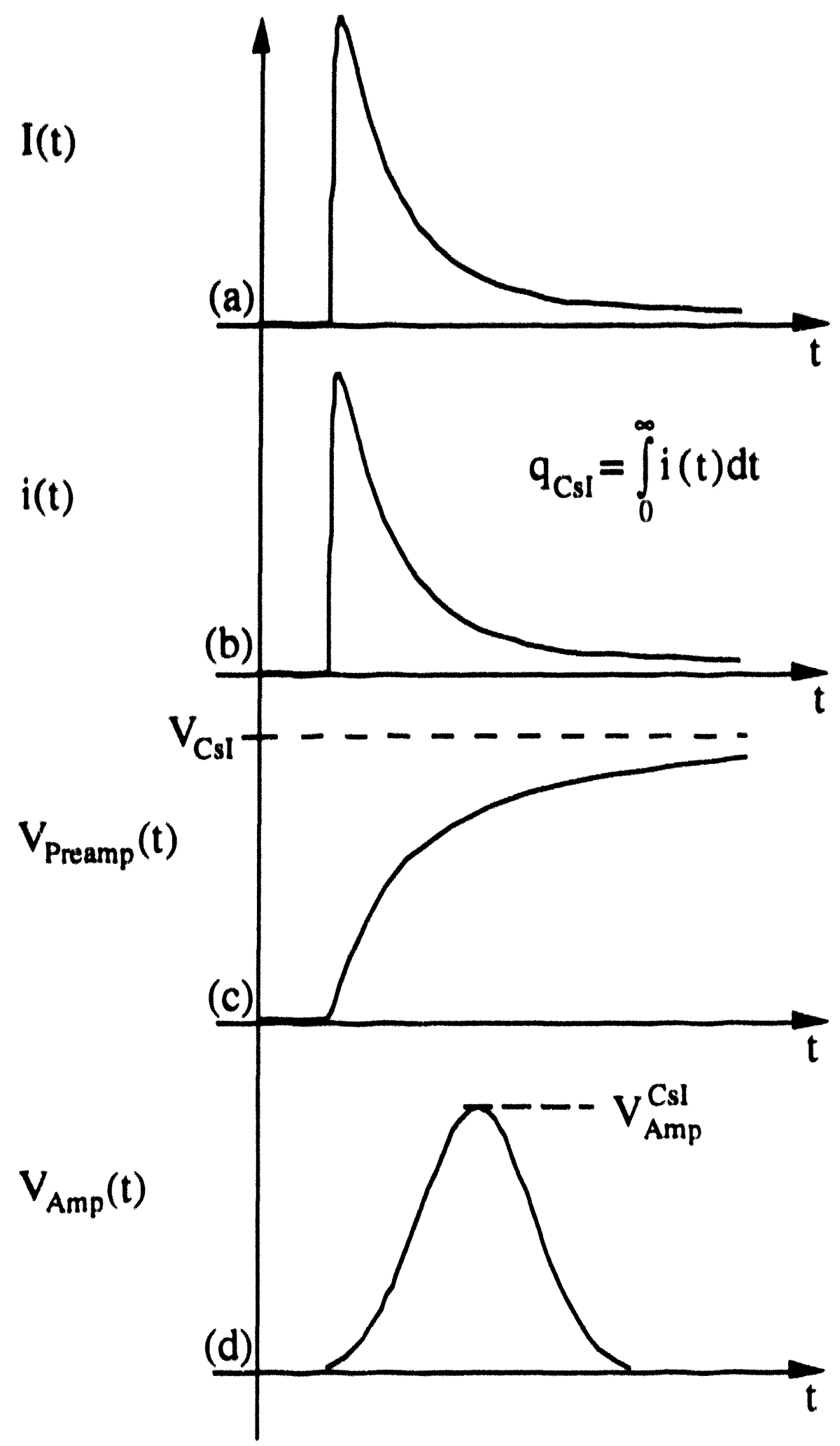

Figure 2.1. CsI(TI) scintillation pulse shapes: (a) scintillation yield, (b) preamplifier input current pulse, (c) preamplifier output voltage pulse, and (d) amplifier output voltage pulse. 
pulse height to integrated charge for systems with PDs (observing direct photon interactions in the PD and introducing a square wave voltage pulse onto the input capacitor.

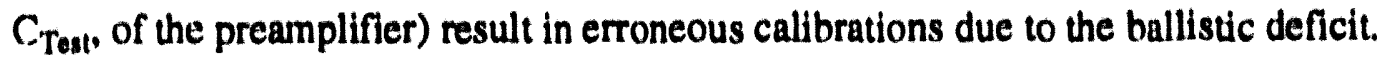

\subsubsection{Direct Interactions in PD}

The use of direct gamma-ray or X-ray interactions in a PD has been a widely-used charge calibration method. It relies on a full energy event, $E_{\gamma}$ in the PD creating $q_{D I}=E_{\gamma} / \varepsilon$ $e-h$ pairs, where $\varepsilon$ is the average deposited energy necessary to create an $e-h$ pair. Although this method accurately calibrates the amplifier pulse amplitude to the charge created by a direct interaction in the PD, the charge is introduced to the preamplifier in a time frame that is short compared to that characterizing a CsI(TI) scintillation event. In this case, the preamplifier output pulse rise time is shor relative to most shaping times and thus not affected by ballistic deficit. Figure 2.2 demonstrates how the ballistic deficit affects the direct interaction calibration. To illustrate the problem, we have assumed that the total charge created in the PD by the direct interaction is the same as the total charge created by a CsI(TI) scintillation event $\left(q_{\mathrm{DI}}=q_{\mathrm{CII}}\right)$. Note that the shaped pulse amplitude of the direct interaction pulse will be nearly constant for all shaping times, while the shaped CsI(TI) pulse amplitude has been previously noted to increase with increasing shaping time. Thus, due to the difference in the preamplifier input current pulse, the direct interaction calibration will be shaping time dependent. Under these circumstances the CsI(TI) scintillation yield inferred from this calibration method will be different for each amplifier shaping time and will underestimate the true yield.

\subsubsection{Square Wave on $\mathrm{C}_{\text {Test }}$}

Introducing a square wave of amplitude $\mathrm{V}_{0}$ onto $\mathrm{C}_{\text {Test }}$ through the test input of the preamplifier will result in an short impulse of charge, $q_{s w}=C_{E_{q}} V_{0}$, that is introduced to the 


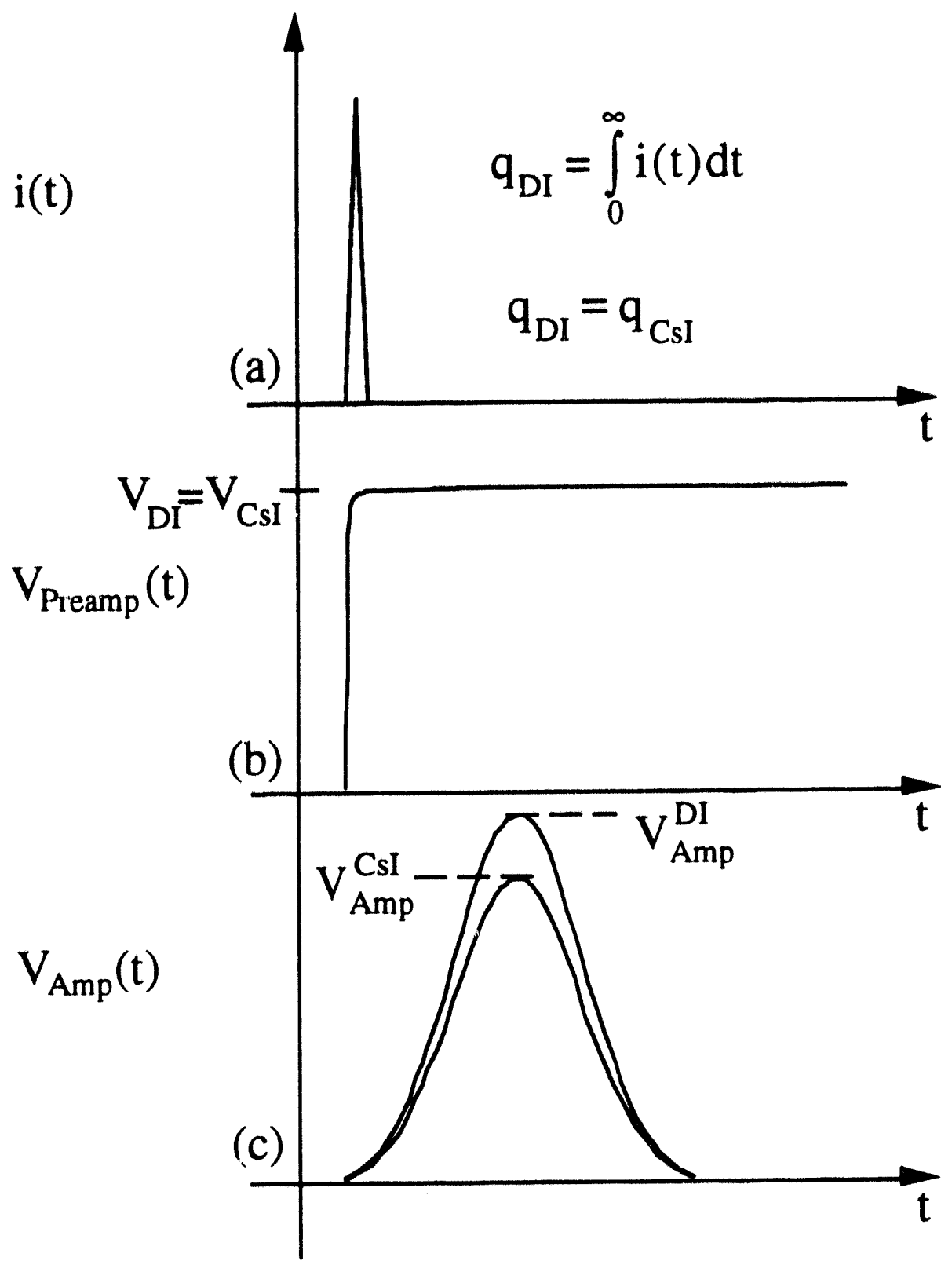

Figure 2.2. Direct interaction (DI) method pulse shapes:(a) preamplifier input current pulse, (b) preamplifier output voltage pulse, and (c) amplifier output voltage pulse.

charge integrator. $\mathrm{C}_{\mathrm{Eq}}$ is the parallel sum of the test capacitance and any stray capacitance $\left(C_{E q}=C_{\text {Test }}+C_{\text {stray }}\right)$. Figure 2.3 demonstrates the pulse shapes for the square wave method when the total charge is equal to the charge created in the PD by a CsI(TI) scintillation event $\left(q_{s w}=q_{C s I}\right)$. Since we again have an impulse of charge, this method produces results similar to the direct interaction method, and the inferred scintillation yield will again depend on the choice of shaping time. 


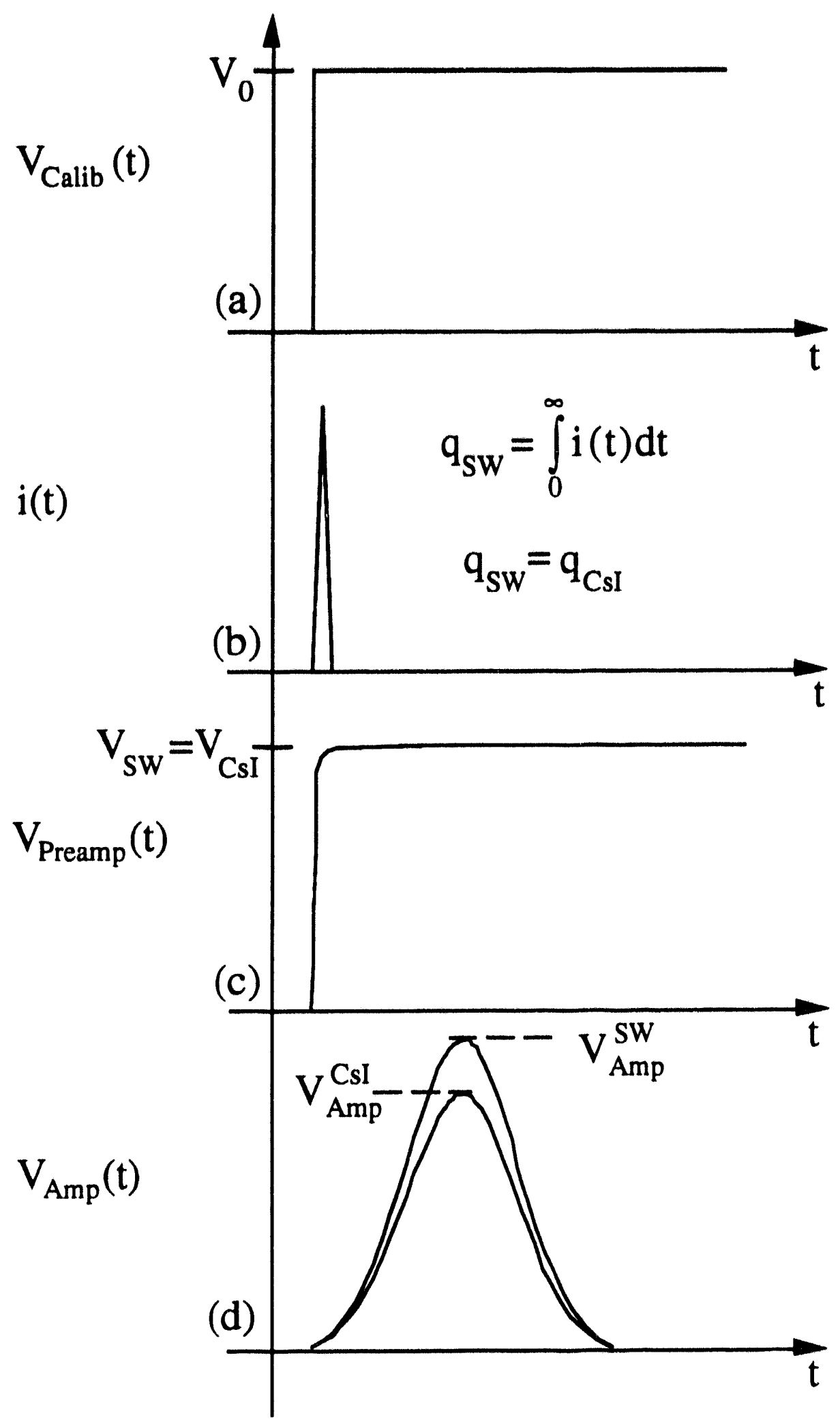

Figure 2.3. Square wave (SW) method pulse shapes: (a) calibration voltage pulse, (b) preamplifier input current pulse, (c) preamplifier output voltage pulse, and (d) amplifier output voltage pulse. 


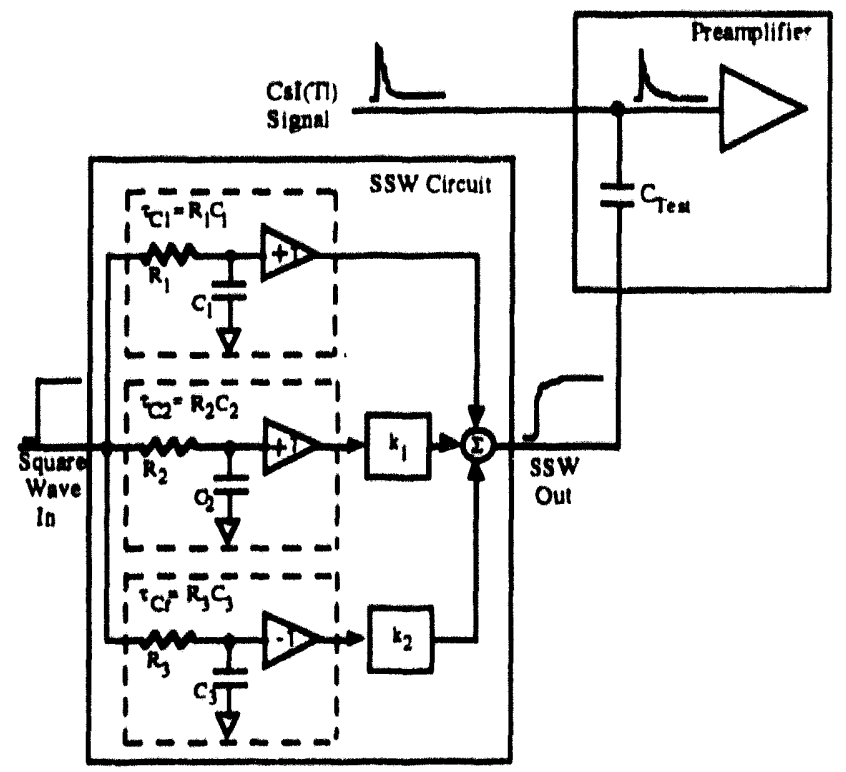

Figure 2.4: Shaped square wave (SSW) method schematic.

The precision to which $\mathrm{C}_{\mathrm{Eq}}$ is known directly affects the precision of the calibration. For the preamplifier used, $\mathrm{C}_{\mathrm{Eq}}$ was inferred from the direct interaction method to assess the contribution of $\mathrm{C}_{\text {stray. }}$. The resulting equivalent capacitance of about $1.27 \mathrm{pF}$ is consistent with the nominal value of the capacitance of the test capacitor $(1.2 \mathrm{pr} \pm 1 \%)$ and the addition of a stray capacitance on the order of a few percent.

\subsubsection{Shaped Square Wave (SSW) on $\mathrm{C}_{\text {Test }}$}

To compensate for the ballistic deficit that is the result of the long decay time of $\mathrm{CsI}(\mathrm{Tl})$, we propose to introduce a current pulse of known total charge, $\mathrm{q}_{\mathrm{ssw}}$, with the shape of the scintillation pulse, Eqn. (2.16), to the charge iniegrator. The shaped square wave (SSW) method models the scintillation pulse to satisfy this requirement [VAL92a]. As seen in Figure 2.4, a square wave is introduced to the SSW circuit, and the pulse is split into three branches, each branch representing one characteristic time ( 2 decays and 1 rise time constant) and the relative contribution of that rise or decay mode to the pulse. To simulate the characteristic time, each branch is passed through an integration stage (two non-inverting for the decay times and one inverting for the rise time) with an RC time 
constant that represents the respective rise or decay time constant. Subsequently, the branches representing $\tau_{2}$ and $\tau_{\text {rise }}$ are attenuated by $k_{1}$ and $k_{2}$, respectively, to model the contribution of the long decay time and the rise time to the pulse, relative to the contribution of $\tau_{1}$. Finally, all three branches are summed resulting in a voltage pulse that when applied to $\mathrm{C}_{\text {Test }}$ will yield a current pulse with the same shape as the $\mathrm{CsI}(\mathrm{Tl})$ scintillation pulse. The negative voltage pulse introduced to $C_{\text {Test }}$ will behave according to

$$
v_{\text {Callb }}(t)=-v_{0}\left(e^{-t / \tau_{c 1}}+k_{1} e^{-t / \tau_{c 2}}-k_{2} e^{-t / \tau_{c t}}\right)
$$

For this input voltage pulse, the current introduced to the charge integrator is

$$
i(t)=C_{E q} \frac{d}{d t} V_{C a l i b}(t)=-C_{E_{q}} V_{0}\left(-\frac{e^{-t / \tau_{C l}}}{\tau_{C 1}}-\frac{k_{1} e^{-t / \tau_{C 2}}}{\tau_{C 2}}+\frac{k_{3} e^{-t / \tau_{C r}}}{\tau_{C r}}\right) \text {, }
$$

while the total charge delivered to the charge integrator is

$$
\mathrm{q}_{\mathrm{Ssw}}=\int_{0}^{\infty} \mathrm{i}(\mathrm{t}) \mathrm{dt}=\mathrm{C}_{\mathrm{Eq}} \mathrm{V}_{0}\left(1+\mathrm{k}_{1}-\mathrm{k}_{2}\right) \text {. }
$$

The resulting preamplifier output voltage pulse will be

$$
V_{\text {Preamp }}(t)=\frac{q_{S S W}}{C_{F}} e^{-t / \tau_{F}}-\frac{C_{E_{q}} V_{0}}{C_{F}}\left[e^{-t / \tau_{C l}}+k_{1} e^{-t / \tau_{C 2}}-k_{2} e^{-t / \tau_{C r}}\right]
$$

Eqns. (2.17) and (2.21) are identical at all times only if $\tau_{1}=\tau_{C 1}, \tau_{2}=\tau_{C 2}, \tau_{\text {rise }}=\tau_{C r}$, $q_{C s l}=q_{s s w}, q_{1}=C_{E q} V_{0}, k_{1}=q_{2} / q_{1}$, and $k_{2}=\tau_{\text {rise }} / \tau_{1}$. Figure 2.5 shows the results of the SSW method when the above conditions are satisfied. With scintillation and calibration pulses of identical shape and amplitude, the calibration of amplifier pulse height to charge created in the PD is independent of shaping time because each amplifier output pulse has the same ballistic deficit.

The trailing edge of the initial square wave will produce exactly the same results as the leading edge but with the opposite sign. The negative pulses will pass through the amplifier, but will not be recognized by the analog-to-digital converter in the multi-channel analyzer.

In summary, the SSW method relies on creating a calibration pulse of known charge with the same shape and amplitude as the full energy CsI(TI) scintillation pulses 


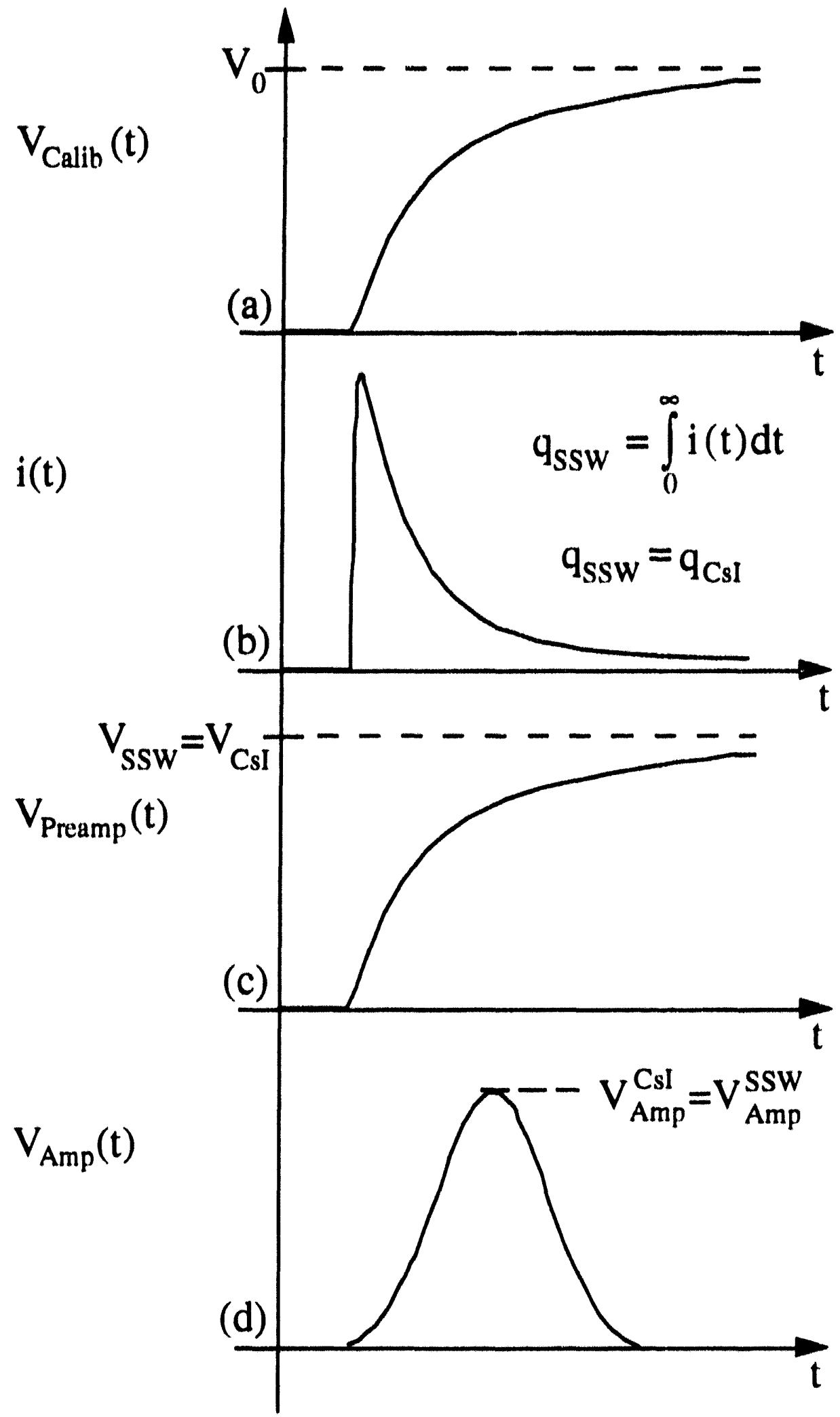

Figure 2.5. Shaped square wave (SSW) method pulse shapes: (a) calibration voltage pulse, (b) preamplifier input current pulse, (c) preamplifier output voltage pulse, and (d) amplifier output voltage pulse. 
such that both pulses experience the same ballistic deficit. When this is realized, the photopeak and calibration peak centroids will occur in the same channel of a multi-channel analyzer, independent of amplifier shaping time. Since the SSW method relies on comparing pulse amplitudes (or integrated charges) for different available shaping times, the method will only be sensitive to rise and decay components that are similar to the amplifier shaping times. Although the SSW method was developed for calibrating pulse amplitude to the integrated charge created in a photodiode by a $\mathrm{CsI}(\mathrm{Tl})$ scintillation pulse to ultimately determine absolute scintillation yield, the method also conveniently results in estimates of the rise and decay time constants and the relative contribution of the luminescence modes. We shall use this feature in the next section.

\subsection{Rise and Decay Time Constant Determination}

The decay time constants and relative intensities of the decay components dictate the high rate capabilities of a scintillator. Thus the $a_{j}{ }^{\prime} s$ and $\tau_{j}$ 's in Eqn. (2.3) and their temperature dependence are important scintillation characteristics that are used to evaluate the suitability of a scintillator for a given application. Consequently, the temporal luminescence intensity of $\operatorname{CsI}(\mathrm{Tl}), \mathrm{I}(\mathrm{t})$, has been determined by both the Bollinger-Thomas method [BOL61], as modified by Moszynski and Bengtson [MOS77], and by the shaped square wave (SSW) method [VAL92a]. The modified Bollinger-Thomas (MBT) method has become the standard technique for determining $I(t)$, and was thus used both to determine the most accurate estimates of the parameters and to confirm the applicability of determining $I(t)$ by the SSW method.

\subsubsection{Modified Bollinger-Thomas method}

The Bollinger-Thomas method was developed specifically for determining luminescence decay times. As moditied, the method uses a trigger that is correlated in time with the initiation of the luminescence signal; a photodetector then randomly samples the 
luminescence distribution by restricting the mean number of photoclectrons generated per trigger to much less than one. This restriction is commonly realized by creating a "poor" crystal-to-photodetector geometry. To determine the true luminescence intensity $I(t)$, standard nuclear electronics, with an impulse response function $G(t)$, are used to acquire a timing spectrum. (The temporal representation of $G(t)$ is discussed in Appendix D.) Consequently, the observed luminescence intensity $\mathrm{H}(t)$ corresponds to the convolution of the impulse response function and the true luminescence intensity:

$$
H(t)=\int_{0}^{\infty} I\left(t^{\prime}\right) G\left(t-t^{\prime}\right) d t^{\prime} .
$$

For fitting purposes $\mathrm{l}(\mathrm{t})$ was normalized to the absolute scintillation yield, $\mathrm{N}_{\text {Abs. }}$ which is the sum of the aj's. With this normalization, each aj represents the fraction of the total light emitted by the $j^{\text {th }}$ process. For the case of an exponential process $\left(a_{\text {rise }}, \tau_{\text {rise }}\right)$ populating a luminescent state $\left(a_{\text {decay }}, \tau_{\text {decay }}\right)$, the fraction of the total light contributed by this rise/decay pair is $a_{\text {rise }}+a_{\text {decay }}$.

The measured data, $M_{k}$, are the number of counts in the $k^{\text {th }}$ bin (corresponding to the time interval $\left.\left[l_{k}, l_{k}+\delta t\right]\right)$ and are analyzed using a non-linear least squares fitting program [DER93]. The fitted number of counts in the $k^{\text {th }}$ bin, $F_{k}$, is determined by summing the integral of the timing spectrum and $R_{c b}$, the chance coincidence rate contribution to that bin:

$$
F_{k}=R_{c h} \delta t+\int_{i_{k}}^{i_{k}+\delta t} H(t) d t
$$

The chance rate appears as a flat background on which the luminescence distribution is superimposed. This manifestation only occurs when the chance rate is much smaller than the inverse of the random sampling time (TAC range). Minimization of $\chi^{2}$, where

$$
\chi^{2}=\sum_{k} \frac{\left(M_{k}-F_{k}\right)^{2}}{\sigma_{k}^{2}}=\sum_{k} \frac{\left(M_{k}-F_{k}\right)^{2}}{F_{k}},
$$

results in the best estimates of the $a_{j}$ and $\tau_{j}$ parameters, where $F_{k}$ is the best estimate of the variance of the counts in the $k^{\text {th }}$ bin. The number of dimensions on the $\chi^{2}$ surface is the 


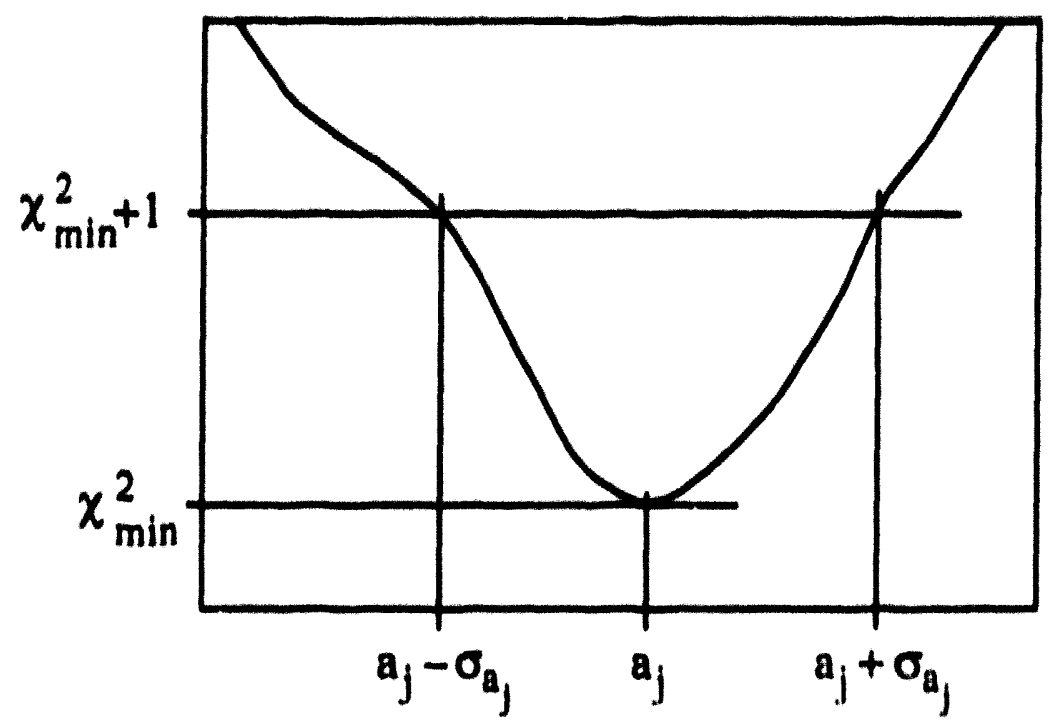

Figure 2.6: $\chi^{2}$ surface projected into a, space.

number of free parameters used in the fitting program, which corresponds to twice the number of exponential components in Egn. (2.3) (one for each $a_{j}$ and $\tau_{j}$ ). plus one each for the flat background or chance coincidence rate $R_{c h}$ and the zero offset of the luminescence pulse (the fixed delay time). As seen in Fig. 2.6, the fit parameter value of aj is chosen at $\chi_{\min }^{2}$. To determine the statistical uncertainties of the fit parameters, the largest and smallest value of each parameter on the $\chi_{\min }^{2}+1$ surface are determined while all other parameters are varied. Fig. 2.6 demonstrates this method for the parameter $a_{j}$, where the $\chi^{2}$ surface has been projected into $a_{j}$ spac?. This method results in values for $+\sigma_{a_{j}}$ and $-\sigma_{a j}$ that are not equivalent when the $\chi^{2}$ surface is asymmetric. When this occurs, the reported value of $\sigma_{a j}$ is the average value of the $+\sigma_{a j}$ and $1-\sigma_{a j} \mid$, such that $a_{j} \pm \sigma_{a j}$ is reported. For most temporal luminescence intensity timing spectra, the values of $+\sigma$ and $-\sigma$ are nearly equal and thus the $\chi^{2}$ surface is approximately symmetric. This method of determining statistical uncertainties is discussed extensively hy Rogers [ROG75]. 
33

2.3.2 Shaped square wave method

The SSW method, described in Section 2.2.3, was developed specifically for the charge calibration of CsI(TI)/photodiode systems to account for the ballistic deficit due to the long decay time constants. However, the method also conveniently result r in estimates of the rise and decay time constants and the relative contribution of the luminescence modes from the $\mathrm{RC}$ time constants and attenuation factors, respectively. The $\mathrm{RC}$ constants $\tau_{\mathrm{Cl}}$, $\tau_{\mathrm{C}_{2}}$, and $\tau_{\mathrm{C}_{r}}$ are estimates of $\tau_{1}, \tau_{2}$, and $\tau_{\text {rite }}$ when the SSW method has been successfully applied, while $k_{1}$ results in an estimate of the ratio of the charge contributions from luminescence mode 2 to mode $1, q_{2} / q_{1}$ or $a_{2} /\left(a_{1}+a_{T 1180}\right)$. As previously mentioned, the SSW method is only sensitive to rise and decay times that are on the order of the amplifier shaping times (250 ns to $12 \mu \mathrm{s})$. 


\section{CHAPTER III EMISSION SPECTRUM}

The emission spectrum of a scintillator can be used to leam about the scintillation mechanisms of that particular material and to determine which photodetector will most efficiently detect that material's scintillation light. Wavelengths of peak emission correspond to the energy lovels of the radiative states for that scintillator, and can thus be used to better understand the solid state physics that govem the behavior of that scintillator. Accurate quantification of the Csl(TI) energy levels requires measuring the fine structure of the emission spectrum. Conversely, to characterize a scintillation material for use as a gamma-ray detector, it is not necessary to accurately determine the fine structure of the emission spectrum. This is because the product of the emission spectrum, $\varepsilon(\lambda, T)$, and quantum efficiency, $Q(\lambda, T)$, is integrated over all wavelengths (Eqn. (2.10)) to determine the wavelength-averaged quantum efficiency, $\bar{Q}(T)$, where $Q(\lambda, T)$ for a $P D$ is a slowly varying function of wavelength. Thus, knowledge of the fine structure of the emission spectrum will not significantly improve the accuracy of the calculated value of $\bar{Q}(T)$. To calculate $\bar{Q}(T)$ by Eqn. (2.10), the emission spectrum must be normalized to unity (Eqn. (2.5)). This normalization and the slowly varying wavelength dependence of the PD quantum efficiency imply that only the coarse shape of the emission spectrum is needed to determine $\overline{\mathbf{Q}}(\mathrm{T})$.

The energy resolution of any detector will be limited by the statistical fluctuations in the number of charge carriers created, $N_{c c}\left(E_{\gamma} T\right)$; thus, the best energy resolution will be achieved by a detector system that creates the most charge carriers per unit energy deposited. For many years it was believed that the CsI(TI) scintillation yield was inferior to 
that of $\mathrm{NaI}(\mathrm{TI})$ and thus inherently had a poorer limiting energy resolution. These beliefs were based on relative measurements of PMT photoelectron yield and did not correct for the wavelength-averaged quantum efficiency. As seen in Figure 1.1, the quantum efficiency of a typical PMT at the peak emission wavelength of CsI(TI) $(560) \mathrm{nm})$ is about an order of magnitude lower than at the peak emission of $\mathrm{NaI}(\mathrm{TI})(450 \mathrm{~nm})$, thus resulting in the erroneous conclusion that the scintillation yield of $\mathrm{Nal}(\mathrm{TI})$ is superior. In contrast to PMTs, the quantum efficiency of a PD increases over the range of $3(x) \mathrm{nm}$ to $960 \mathrm{~nm}$. This range of increasing quantum efficiency includes the emission wavelengths of both NaI(TI) and CsI(TI), thus the wavelength-averaged quantum efficiency for CsI(TI) will he superior Io that of NaI(TI) when hoth crystals are coupled to a PD. Furthermore, we can see from Figure 1.1 that the wavelength-average quantum efficiency for a $\mathrm{CsI}(\mathrm{TI}) / \mathrm{PD}$ detector will the superior to that of the CsI(TI)/PMT. Nal(TI)/PMT, and Nal(TI)/PD combinations. Thus a PD will he the photodetector of choice for most applications that utilize CsI(TI) at room temperature, while a PMT is still the hest photodelector when using Nal(TI). Prior to the development of silicon PIN PDs, the NaI(TI)/PMT combination yielded the largest number of charge carriers per unit energy deposited of any scintillator/photodetector pair (at hest (1).(XK) photoelectrons/MeV) and thus had the hest possihle energy resolution. With the present commercially-availahle PDs, the CsI(TI)/PD combination now yields at least four times as many charge carriers (ahout 4()$, 0(X)$ electron-hole pair. $M e V$ ) and has hecome the scintillator/photodetector with the hest possible energy resolution.

Changes in the shape of the Csl(TI) emission spectrum with temperature will change the wavelength-averaged quantum efficiency and could thus change the photodetector preference. Therefore, it is important to accurately know the shape of the cmission spectrum as a function of temperature so that an appropriate photodetector can he chosen and the wavelength-averaged quantum efficiency can be calculated at the anticipated lemperature of operation. 
As stated in Chupter I, the CsI(TI) emission spectrum and its temperature dependence have been reported by several authors. In most cases it is not apparent whether the emission spectra were corrected for the spectral efficiency of the detection system used. Not correcting for the spectral efficiency can lead to very poor representations of the emission spectrum. For example, Schotanus, et al. [SCH90) report CsI(TI) emission spectrum data that was not corrected for the spectral efficiency of the monochromator and PMT and the resulting emission spectrum is peaked at about $5(0) \mathrm{nm}$. The authors acknowledge that the emission spectrum probably peaks at about $560 \mathrm{~nm}$. However, if their data were used to calculate the wavelength-averaged quantum efficiency, a result at least 5\% smaller than the expected value for CsI(TI) coupled with a iypical silicon PIN PD would the realized. Therefore, correcting emission spectra data for the spectral efficiency of the detection system is an essential part of the ahsolute measurement of $e(\lambda, \mathrm{T})$ covered in this chapter.

\subsection{Emisalon Spectrum Experimental Methods}

The CsI(TI) crystals procured from Bicron Corporation. Horiha Crystal Products, Rexon Components, Inc., and Solon Technologies, Inc. (STI) were used to assess the crystal-10-crystal variations and temperature dependence of the emission spectrum; the STI crystal was randomly chosen for the temperature dependence measurements. As seen in Fig. 3.1, the emission spectra of the four CsI(TI) crystals were measured using a 0.125 meter Jarrell-Ash MonoSpec 18 monochromator with two different $12(x)$ line/mm gratings, one blazed for $3(X) \mathrm{nm}$ and the other for $s(x) \mathrm{nm}$. The monochromator entrance and exit slits were $5(0) \mu \mathrm{m}$ wide, which corresponds to a $12 \mathrm{~nm}$ spectral resolution. $511 \mathrm{keV}$ annihilation photons from a $0.7 \mathrm{mCl}$ toge source were used to excite the crystals. The 12 $\mathrm{nm}$ spectral resolution is not sufficient for determining the fine structure of the CsI(TI) umission spectrum, hut is sufficient for identifying the major variations that affect the calculation of the wavelength-averaged quantum efficiency. The crystal of interest was 


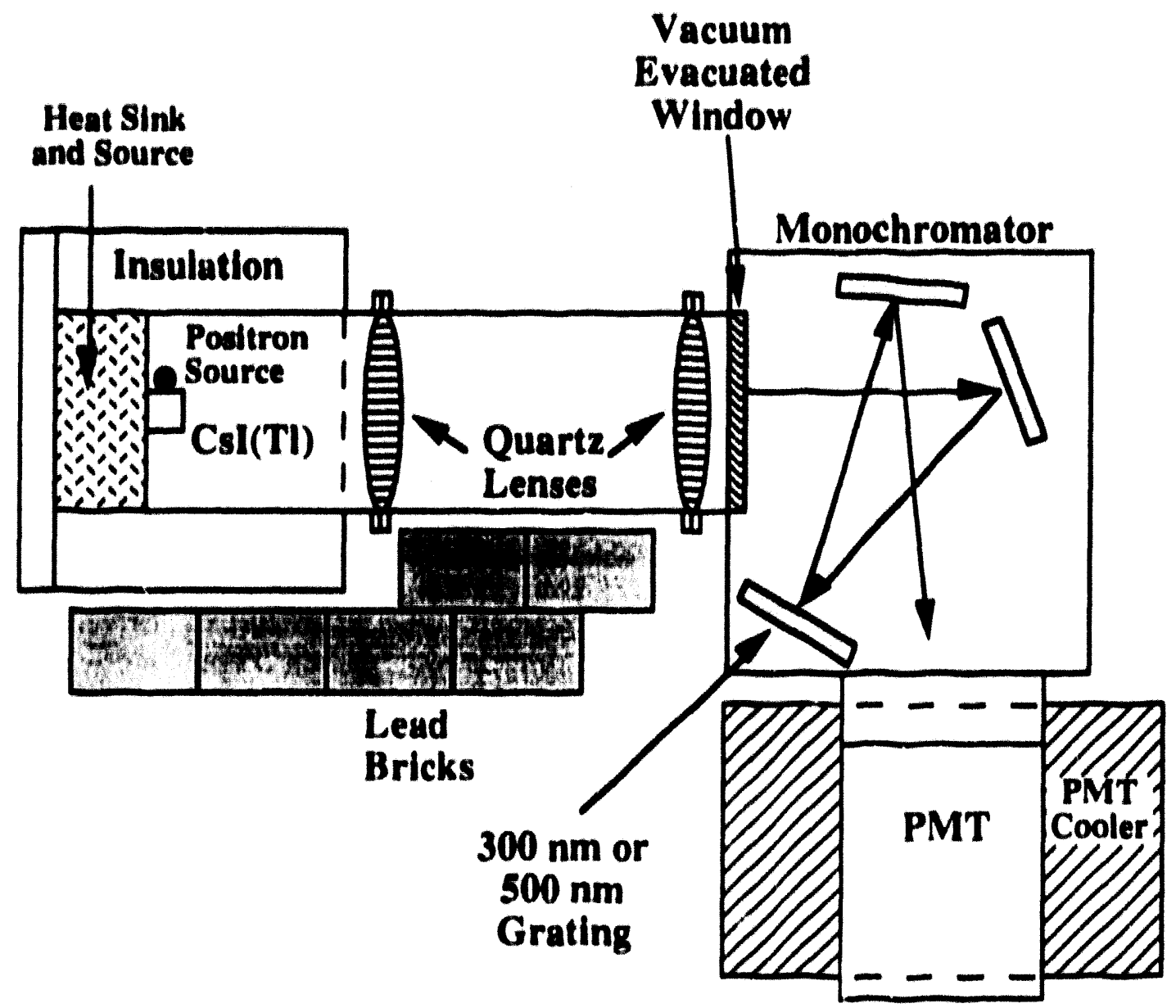

Figure 3.1: Detection system schematic for emission spectra measurement.

placed at the focal point of a quartz lens; a similar lens then refocused the emissions onto the entrance slit of the monochromator. The crystals and first focusing lens were placed in a separate, thermally-insulated, temperature-controlled enclosure that was optically coupled to the PMT via quarz lenses and air gaps. A brass block served as the heat sink and souree for the enclosure. To allow low temperature operation, a constant flow of liquid nitrogen was passed through the block. The temperature regulation was provided by a microprocessor-controlled heating element in the brass hlock (temperature regulation method $A$ ) as shown schematically in Fig. 3.2. The crystal temperature was monitored by placing a thermocouple in direct contact with the crystal. A Hamamatsu R-2059 PMT was placed at the exit slit of the monochromator in a thermally insulated enclosure. The PMT 


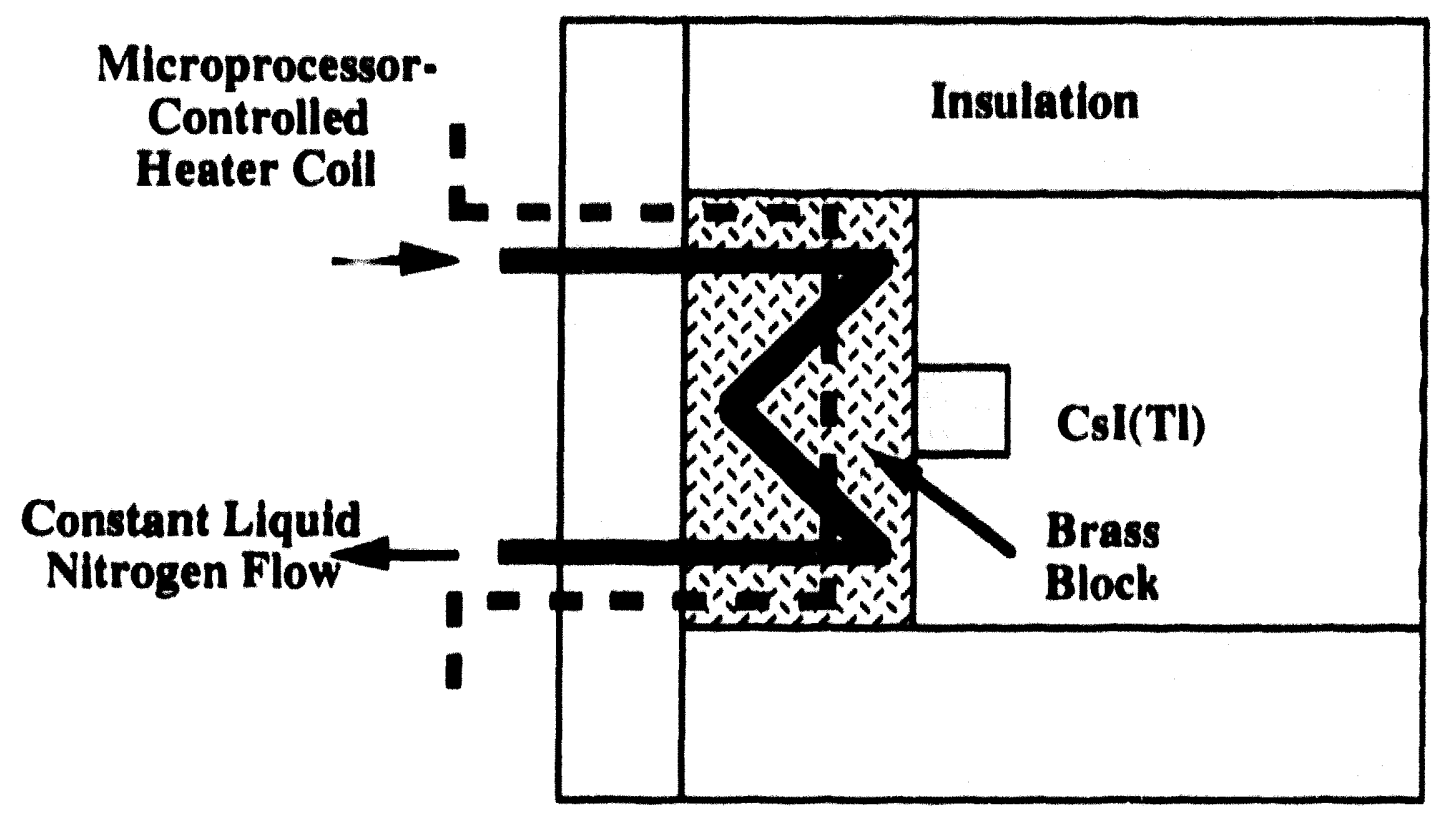

Figure 3.2: Temperature regulation method $A$

was cooled to $-20^{\circ} \mathrm{C}$ to reduce the dark current, and a background rate of about 10 photoelectruns/second was thus achieved. The PMT was shielded from the source with lead bricks to prevent direct interactions of the $511 \mathrm{keV}$ photons in the PMT. The output of the PMT was converted to a logic pulse by a Tennelec TC.454 constant fraction discriminator and subsequently counted with a Jorway 84 CAMAC scaler (Fig. 3.3). To determine the emission spectra, count rate data were acquired for 30 seconds at each $5 \mathrm{~nm}$ increment over the range of 200 to $7(0) \mathrm{nm}$ and were corrected for the background count rate due to dark current. Data were acquired over the range of 200 to $500 \mathrm{~nm}$ with the grating blazed for $300 \mathrm{~nm}$, while similar acquisitions were made from $3(10$ to $700 \mathrm{rm}$ with the $500 \mathrm{~nm}$ grating. The data taken with each grating were corrected for the spectral efficiency of the monochromator and PMT.

To determine the spectral response of the monochromator and PMT, the temperature chamber and crystal were replaced with a NIST traceable, calibrated Hamamatsu L2196 Deuterium Lamp. The known emission of the lamp, normalized to unity at peak emission, and the observed response of the system for both the $300 \mathrm{~nm}$ and 


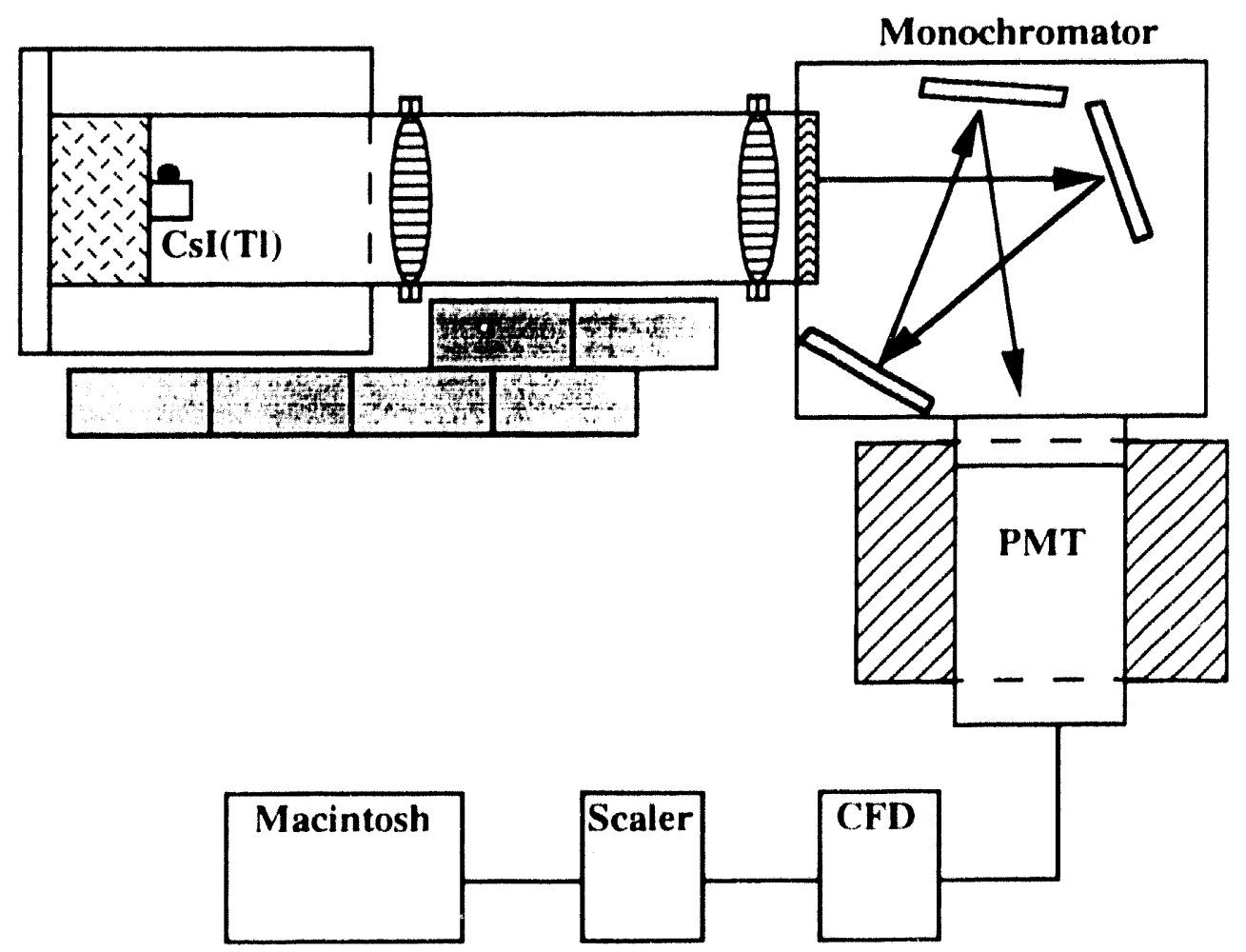

Figure 3.3: Detection system and electronics schematic for emission spectra measurement.

$5(x) \mathrm{nm}$ gratings, normalized to always be smaller than the normalized known intensity of the lamp, are shown in Fig. 3.4 over the range of 20() to $7(0) \mathrm{nm}$ in $5 \mathrm{~nm}$ increments. Much of the line structure of a typical deuterium lamp emission spectrum is lost hecause of the $12 \mathrm{~nm}$ spectral resolution of the monochromator. However, sharp peaks at about 480 and 66() $\mathrm{nm}$ and a hroad peak with a maximum at ahout $580 \mathrm{~nm}$, as well as the other general features of a typical deuterium lamp emission spectrum seen in Fig 3.4, indicate that the emissions of the calibrated lamp are consistent with the expected behavior of such a lamp. Two calihrated cut-off filters were used to assure that the ohserved response of the monochromator and PMT were not contaminated hy higher order passage of light through the monochromator. The actual and ohserved lamp emission spectra have heen normalized such that the relative spectral efficiency of the monochromator and the PMT can he determined for each grating hy dividing the observed response of the system hy the known emission of the lamp at each $5 \mathrm{~nm}$ increment. The resulting relative spectral efficiency for 


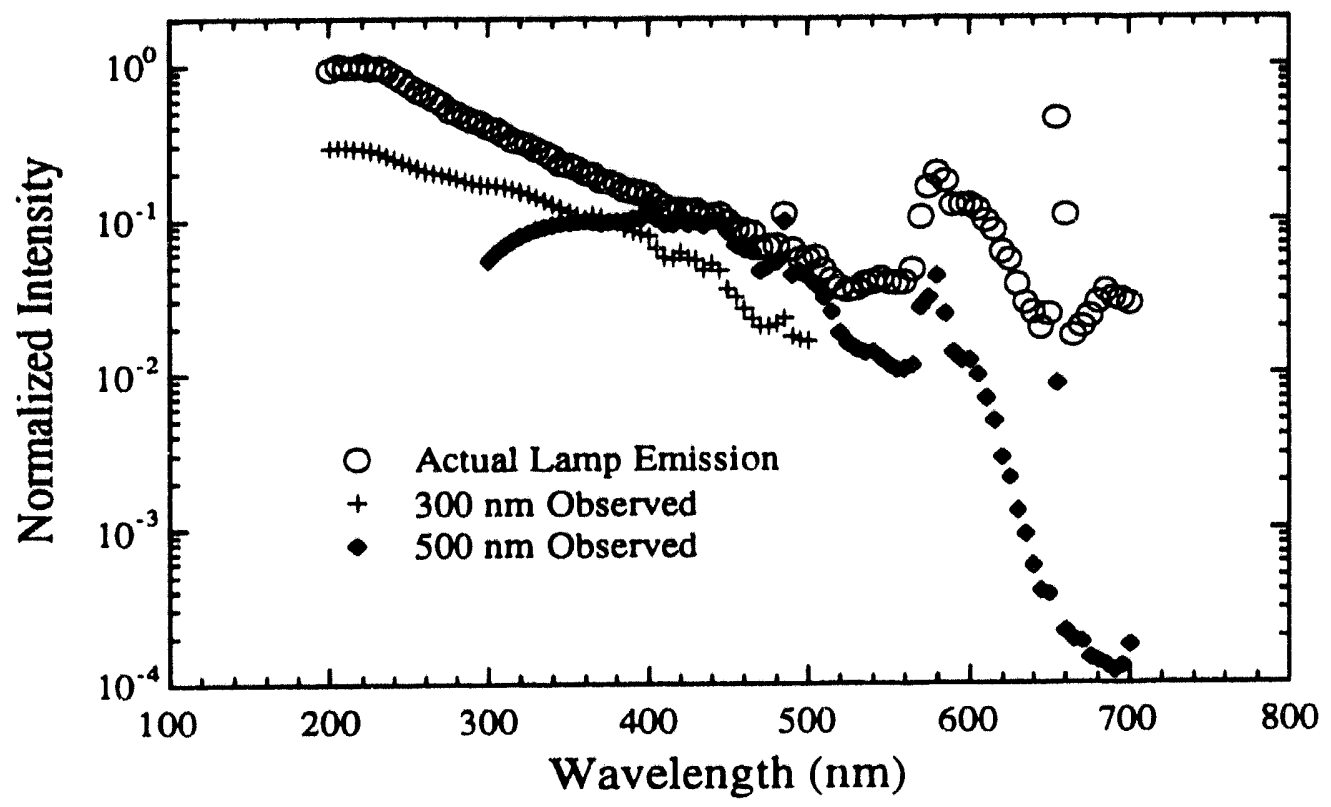

Figure 3.4: Monochromator and PMT spectral efficiency calibration for the 300 and $500 \mathrm{~nm}$ gratings.

the 300 and $500 \mathrm{~nm}$ gratings is shown in Fig. 3.5. The absolute spectral efficiency at each $5 \mathrm{~nm}$ increment is some fraction of the reported values. Since the CsI(Tl) emission spectrum is normalized to unit area when determining the wavelength-averaged quantum efficiency, it is not necessary to know the spectral efficiency absolutely. The normalizations of the actual and observed lamp emissions are irrelevant as long as the same normalization is used for both monochromator gratings. The spectral efficiencies are reported over the range of 200 to $500 \mathrm{~nm}$ and 350 to $700 \mathrm{~nm}$ for the 300 and $500 \mathrm{~nm}$ gratings, respectively, because the emission spectra data for the CsI(Tl) crystals were taken in these ranges. The point-to-point results seen in Fig. 3.5 are smooth with the exception of wavelengths around $480 \mathrm{~nm}$ for the $300 \mathrm{~nm}$ grating and around $430 \mathrm{~nm}$ for the $500 \mathrm{~nm}$ grating. The former is probably due to the emission peak of the lamp at about $480 \mathrm{~nm}$, while the latter may be due to second order contamination. Data acquired with the $300 \mathrm{~nm}$ grating and corrected for spectral efficiency should be reliable over the entire range of wavelengths because the efficiency varies smoothly and never approaches zero. Similarly, 


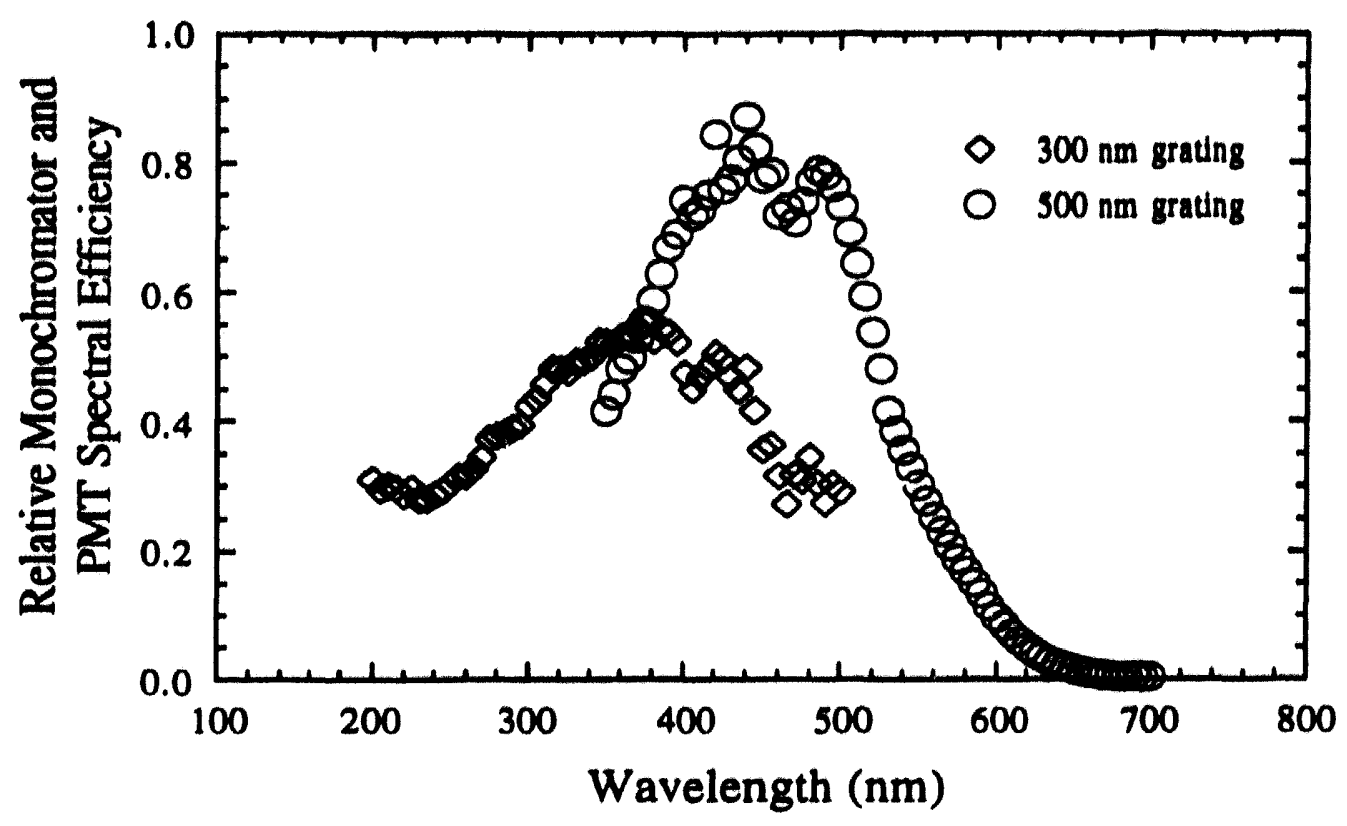

Figure 3.5: Relative spectral efficiency of monochromator and photomultiplier tube for 300 and $500 \mathrm{~nm}$ gratings.

the data acquired with the $500 \mathrm{~nm}$ grating should be very reliable up to $600 \mathrm{~nm}$ and have somewhat decreasing reliability above $600 \mathrm{~nm}$ as the efficiency approaches zero. The spectral efficiency above about $640 \mathrm{~nm}$ is very small (between 0.004 and 0.025 ); this will tend to accentuate fluctuations in the data taken at these wavelengths when the data are corrected for spectral efficiency. The poor spectral efficiency above $640 \mathrm{~nm}$ also manifests itself in poor counting statistics which will result in large fluctuations in the data before it is corrected. Therefore, large fluctuations in the reported emission spectra are expected for wavelengths larger than $640 \mathrm{~nm}$.

\subsection{Emission Spectrum Results}

An example of the background subtracted data taken with each grating for the STI crystal and the data corrected for spectral efficiency at each $5 \mathrm{~nm}$ increment is shown in Fig. 3.6 where any background subtracted data that results in a negative count rate has been omitted. Similar to the uncorrected results of Schotanus, et al. [SCH90], the $500 \mathrm{~nm}$ 


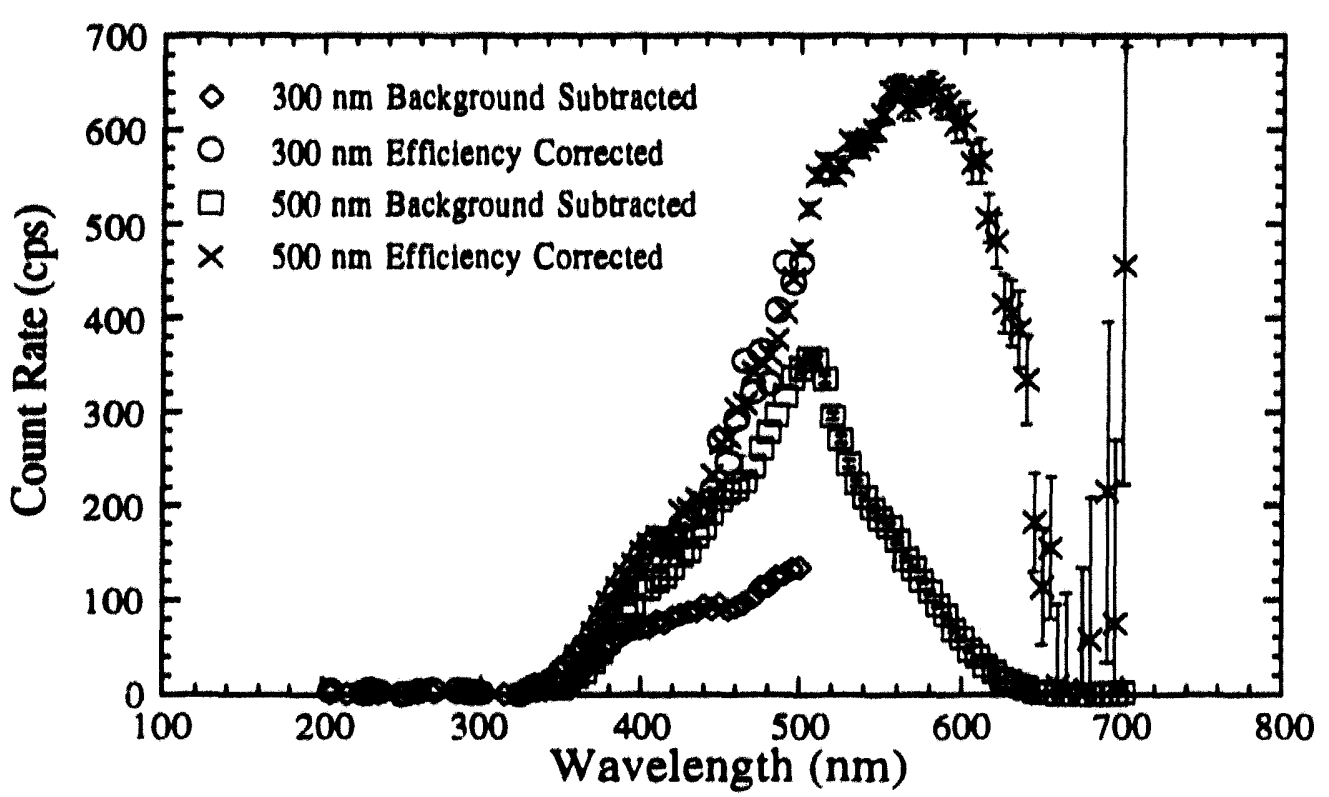

Figure 3.6: Example of background-subtracted and background-subtracted, efficiencycorrected emission spectra data for 300 and $500 \mathrm{~nm}$ gratings.

grating, background subtracted, uncorrected data is peaked at about $500 \mathrm{~nm}$. However, when the data are corrected for the spectral efficiency of the monochromator and PMT, the emission spectrum is observed to peak at about $570 \mathrm{~nm}$, which is similar to previously reported values [CRA74, GRA84, GRA85, GUT74, GWI63, KRE87, LAG61, MAS66, TOW68]. Once corrected for the spectral efficiency, the 300 and $500 \mathrm{~nm}$ grati.ng data agree quite well in the overlapping range of 350 to $500 \mathrm{~nm}$. This agreement confirms the spectral efficiencies derived above. As predicted, the corrected data fluctuates drastically above 640 $\mathrm{nm}$ due to poor counting statistics and poor spectral efficiency. The four data points above $660 \mathrm{~nm}$ that are significantly above zero count rate in the background subtracted, corrected data do not appear to deviate significantly from the other background subtracted, uncorrected data. Similar points that are not shown in the figure appear below zero count rate. These significant fluctuations in the resulting emission spectrum are due to dividing the background subtracted data by the small spectral efficiency of the monochromator and PMT at long wavelengths, as mentioned above. To avoid erroneous results when 
calculating $\bar{Q}(\mathrm{~T})$, data points above $640 \mathrm{~nm}$ that fluctuate significantly from the apparent trend have been omitted and have been likewise omitted from the subsequently reported emission spectra.

A comparison of the CsI(TI) emission spectrum from the four different crystals at room temperature is shown in Fig. 3.7 with the data normalized to unity at the peak emission count rate. The uncertainties shown for the STI crystal are due only to counting statistics and are typical for all emission spectra reported. The emission spectrum for all four crystals is observed to peak between 560 and $570 \mathrm{~nm}$, while a much smaller emission band is observed to peak at about $400 \mathrm{~nm}$. The 400 and $560 \mathrm{~nm}$ emission bands have been attributed to $\mathrm{F}$ centers created by $\mathrm{I}^{-}$vacancies and thallium luminescence centers, respectively [GUT74, SCH90]. Although these are common scintillation processes (as described in Chapter I), it is difficult to verify that these are the actual mechanisms that are producing light. The peak wavelengths indicate that the luminescence energy levels for $F$ center- $\mathrm{V}_{\mathrm{k}}$ center recombination (Fig. 1.5) and for thallium luminescence center recombination (mechanism $C$ in Fig. 1.4) are 3.1 and $2.2 \mathrm{eV}$, respectively, but the $12 \mathrm{~nm}$ spectral resolution of the monochromator is probably obscuring the fine structure of the emission spectrum. Lagu and Thosar [LAG61] reported four peaks in the emission spectrum of CsI(Tl) over the same range of wavelengths. Due to the spectral resolution of our system, three of these four peaks are probably being averaged together to result in the observed broad emission band peaked at $560 \mathrm{~nm}$, while the other previously reported peak is probably the $400 \mathrm{~nm}$ emission band. Furthermore, the data reported here is not sufficient to fully characterize the energy levels and scintillation mechanisms of CsI(Tl). Although the $400 \mathrm{~nm}$ emission band of the Horiba Crystal Products crystal has a slightly smaller magnitude than the other three, all four crystals display very similar room temperature emission spectra. As seen in Table 3.1, when the four emission spectra are averaged over the internal and external quantum efficiency of a typical silicon PIN PD, the resulting wavelength-averaged quantum efficiencies vary by $0.15 \%$ or less from the mean. 


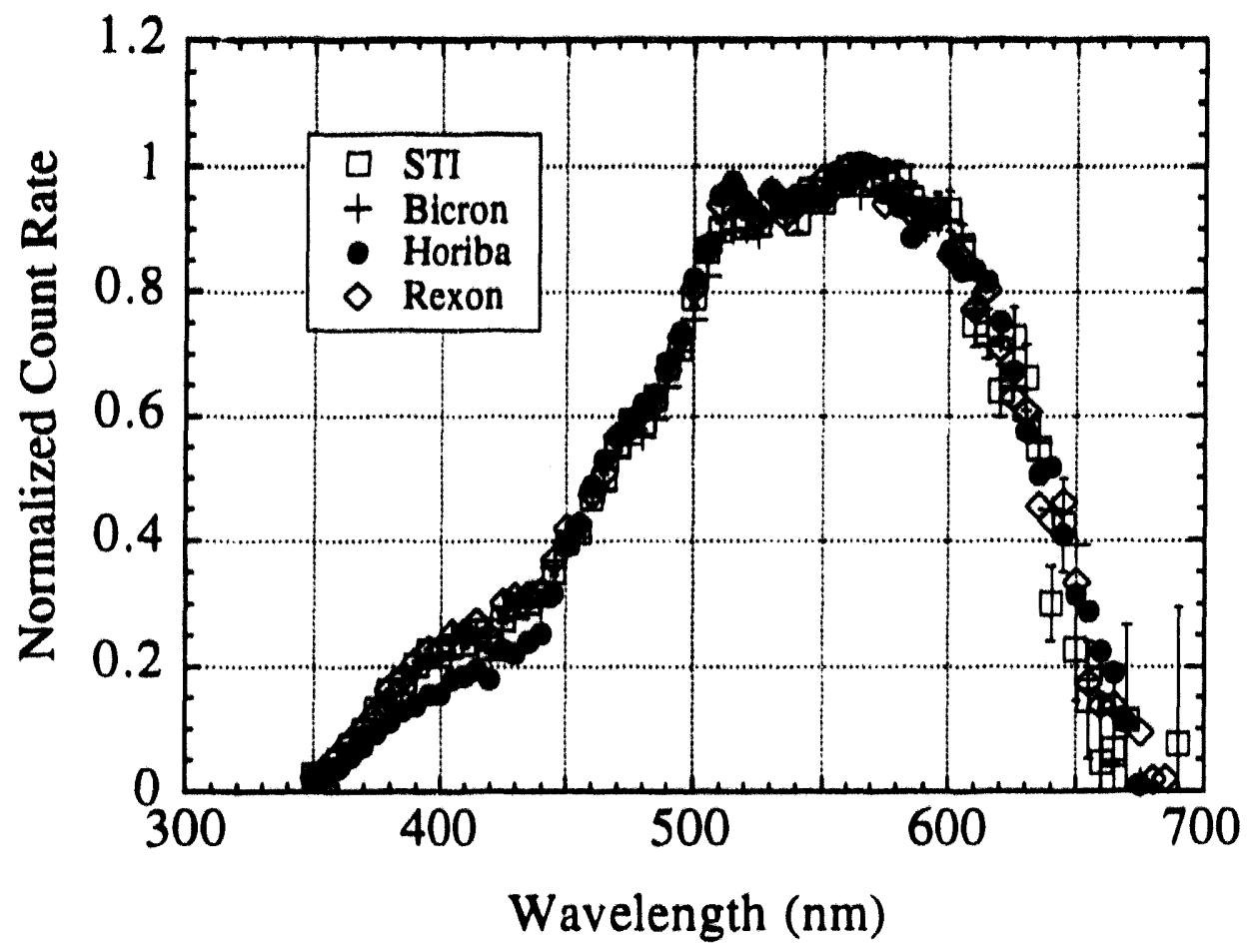

Figure 3.7: Gamma-ray excited CsI(Tl) emission spectrum at room temperature for crystals from Bicron Corporation, Horiba Crystal Products, Rexon Components, Inc., and Solon Technologies, Inc.

Table 3.1: Room temperature internal and external wavelength-averaged quantum efficiencies for a typical silicon PIN photodiode coupled to CsI(Tl) crystals from Bicron Corp., Horiba Crystal Products, Rexon Components, Inc., and Solon Technologies, Inc.

\begin{tabular}{|c|c|c|c|c|}
\hline Crystal & Bicron & Horiba & Rexon & STI \\
\hline Internal $\overline{\mathrm{Q}}\left(25^{\circ} \mathrm{C}\right)$ & 0.896 & 0.898 & 0.895 & 0.897 \\
\hline External $\overline{\mathrm{Q}}\left(25^{\circ} \mathrm{C}\right)$ & 0.685 & 0.689 & 0.684 & 0.687 \\
\hline
\end{tabular}

Consequently, it can be concluded that variations in the emission spectrum of CsI(TI) from vendor to vendor or crystal to crystal at room temperature are minimal. For our definitions of light collection efficiency and quantum efficiency, the internal $\bar{Q}(T)$ is the parameter of interest. We have also included the external values so that our results can be compared to what is expected from the conventional definitions. The small peak that appears in most of the emission spectra at about $520 \mathrm{~nm}$ is believed to be due to the recorded count rate peaking sharply around this wavelength (as seen is Fig. 3.6). Thus, this peak is not 
believed to be a true emission peak, but an aberration of the correction for spectral efficiency.

Since the crystal-to-crystal variations in the CsI(Tl) emission spectrum at room temperature were found to be minimal, we only studied the emission spectrum temperature dependence of a single crystal and assumed that other crystals would behave similarly. Fig. 3.8 shows the emission spectrum of the STI crystal over the range of -100 to $+50^{\circ} \mathrm{C}$ in $25^{\circ} \mathrm{C}$ increments, normalized to the peak emission count rate at room temperature. The emission spectrum appears to peak between 560 and $570 \mathrm{~nm}$ at all ternperatures, while the $400 \mathrm{~nm}$ band is observed to disappear between -50 and $-75^{\circ} \mathrm{C}$. We believe that the decreased mobility of the $V_{k}$ centers that recombine with $F$ centers to create the $400 \mathrm{~nm}$ emission band with decreasing temperature and/or the decreased thermal excitation needed to liberate the electron from the $\mathrm{F}$ center account for the disappearance of this band. Although the $400 \mathrm{~nm}$ band is quite evident above $-50^{\circ} \mathrm{C}$, its relative contribution to a pulse will be small when CsI(Tl) scintillation light is detected with a PD. This small relative contribution is due to the relatively small contribution of the $400 \mathrm{~nm}$ light to the total emission, and also due to the PD quantum efficiency increasing with wavelength in this range. Thus the PD will be more sensitive to photons from the $560 \mathrm{~nm}$ band than the 400 $\mathrm{nm}$ band. Table 3.2 shows the expected temperature dependence of the internal and external wavelength-averaged quantum efficiency for a typical silicon PIN PD coupled to CsI(Tl). All of the $\bar{Q}(\mathrm{~T})$ values reported in Table 3.2 were calculated using the room temperature quantum efficiency and thus do not represent exact values of $\bar{Q}(T)$ but are presented as an indicator of the variations in the emission spectrum with temperature. The implications of these $\bar{Q}(\mathrm{~T})$ values are further discussed at the end of Section 4.3. The increase in $\bar{Q}(T)$ with decreasing temperature is due to the decrease in the number of photons emitted in the $400 \mathrm{~nm}$ band, thus increasing the weight of the $560 \mathrm{~nm}$ band. Due to the normalization of the data in Fig. 3.8, the change in relative peak count rate observed 

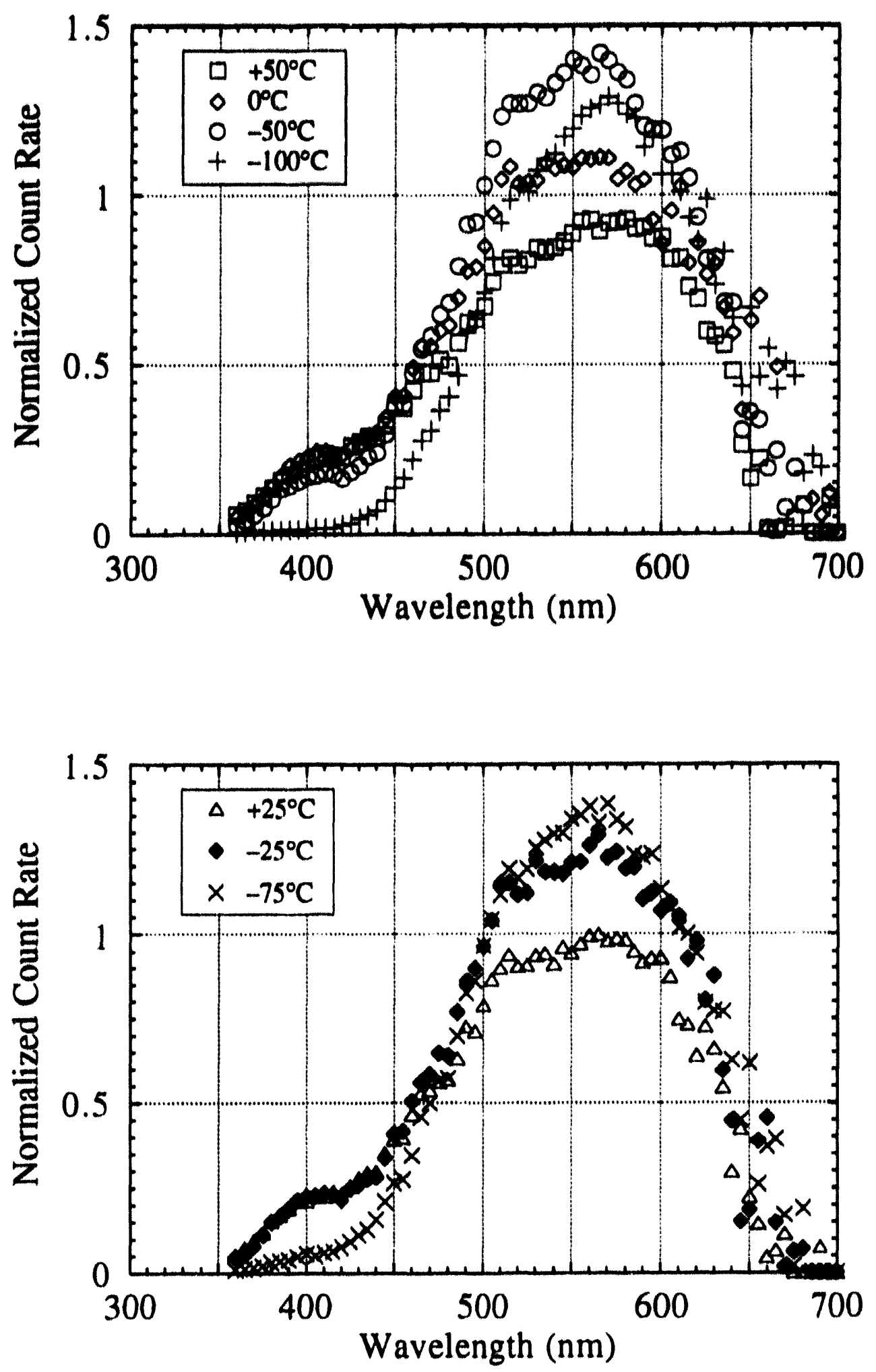

Figure 3.8: Temperature dependence of gamma-ray excited CsI(TI) emission spectrum for Solon Technologies, Inc. crystal. 
Table 3.2: Temperature dependence of wavelength-averaged quantum efficiency for a typical silicon PIN photodiode coupled to the Solon Technologies, Inc. CsI(TI) crystal.

\begin{tabular}{|c|c|c|}
\hline $\begin{array}{c}\text { Temperature } \\
\left({ }^{\circ} \mathrm{C}\right)\end{array}$ & $\begin{array}{c}\text { Internal } \\
\bar{Q}(\mathrm{~T})\end{array}$ & $\begin{array}{c}\text { Extemal } \\
\bar{Q}(\mathrm{~T})\end{array}$ \\
\hline+50 & 0.896 & 0.685 \\
\hline+25 & 0.897 & 0.687 \\
\hline 0 & 0.898 & 0.689 \\
\hline-25 & $0.900)$ & 0.691 \\
\hline-50 & 0.9012 & 0.694 \\
\hline-75 & 0.9017 & 0.702 \\
\hline-100 & 0.912 & 0.709 \\
\hline
\end{tabular}

with changing temperature is indicative of the temperature dependence of the scintillation yield. The reponed relative count rate may he complicated hy $\mathrm{CsI}(\mathrm{TI})$ thermoluminescence emissions, as will be discussed in Chapter VII. Furthermore, the change in relative peak count rate and the area under the emission spectrum are only indicators of the scintillation yield hehavior. However, the shape of the emission spectrum is the scintillation characteristic of interest since $\varepsilon(\lambda, T)$ is normalized according to Eqn. (2.5), and thermoluminescence was not observed to affect the shape of the emission spectrum. Furthermore, using a PD to detect CsI(TI) scintillation emissions will result in the maximum number of charge carriers created at all temperatures because of the minimal variations in the emission spectrum shape and the resulting wavelength-averaged quantum efficiency with temperature.

\subsection{Temporal Dependence of Emission Spectrum}

Spect:ally-resolved timing measurements were made to determine the dependence of the temporal luminescence intensity of $\mathrm{CsI}(\mathrm{TI}), \mathrm{I}(\mathrm{T}, \mathrm{t})$, on the wavelength of the emilted scintillation light, as addressed in Eqns. (2.2) through (2.4). With the monochromator sel at either $4(X)$ or $560 \mathrm{~nm}$, the modified Bollinger-Thomas method (described in Chapter V) 
was used to measure I(T,I) at room temperature. Only a few percent of the emitted CsI(TI) scintillation photons are passed through the monochromator, while the chance coincidence rate will remain approximately the same. The algorithm used to analyze the standard timing spectra could not be used due to this degradation of signal-to-noise. However, no appreciable differences were detected by a visual comparison of the timing spectra acquired from the $400 \mathrm{~nm}$ band, the $560 \mathrm{~nm}$ band, and the entire emission spectrum of CsI(TI). Consequently, we have assumed that the temporal luminescence intensity and emission specirum of CsI(TI) are independent. As previously mentioned, Lagu and Thosar [L.AG61] reported four emission bands. The two most prominent bands peak at ahout 565 and $595 \mathrm{~nm}$ and have similar peak amplitudes. It is possible that those two emission hands are responsible for the two primary luminescence modes and that the fraction of light emitted in the other two bands is so small that the decay times are ohscured in the timing spectrum. Although the decay times were not resolved hy the spectrally.resolved timing measurements, it seems likely that different emission hands would have different decay times. If each emission band has a different temporal hehavior $\left(\varepsilon_{j}(\lambda, T) \neq \varepsilon(\lambda, T)\right)$, then the integration over time and wavelength of Eqn. (2.1) results in

$$
N_{\mathrm{ecc}}\left(E_{\gamma}, T\right)=E_{\gamma} \eta \sum_{j} a_{j}(T) \bar{Q}_{j}(T),
$$

where $\bar{Q}_{j}(T)$ is the effective wavelength-averaged quantum efficiency of the $j^{\text {th }}$ luminescence mode:

$$
\bar{Q}_{j}(T)=\int_{0}^{\varepsilon_{j}}(\lambda, T) Q(\lambda, T) d \lambda .
$$

If $\bar{Q}_{j}(T)$ is approximately constant for all luminescence modes $\left(\bar{Q}_{j}(T)=\bar{Q}(T)\right.$ ), then Eqn. (3.1) is equivalent to Eqn. (2.11). Even if $\bar{Q}_{j}(T)$ is only constant for the primary luminescence modes (primary $a_{j}>$ non-primary $a_{j}$ ) assuming that $\bar{Q}_{j}(T)=\bar{Q}(T)$ will not introduce much error. Since about 90\% of the CsI(TI) scintillation light is emilted in the $560 \mathrm{~nm}$ hand as seen in Fig. 3.7 (or a group of a couple of hands with peak wavelengths 
(lose together), the two primary luminescence modes apparently have similar values of $\bar{Q}_{1}(T)$. Thus, the assumption of the temporal independence of the CaI(TI) emission spectrum $(\varepsilon, \lambda, T)=\ell(\lambda, T))$, and consequendly the wavelength-averaged quantum efficiency, should not introduce much error into the absolute scintillation yield determinations described in Chapter IV. 


\section{CHAPTER IV \\ SCINTILLATION YIELD}

The long decay consunt of CaI(TI) complicates the measurement of the absolute scintillation yield. As seen in Table 1.1, $t_{2}$ is expected to be on the order of a few microseconds at room temperature and to increase with decreasing temperature. While an amplifier shaping ume at least four times longer than the longest scintillation decay time is necessary to preserve the full pulse amplitude through the shaping and amplification process, the largest known commercially-available amplifier shaping time is only $12 \mu \mathrm{s}$. When this condition is not satisfled, the implifier output pulse amplitude is degraded by hallistic deficit and will yield an incorrect value for the scintillation yield. As seen in Eqn. (2.11),

$$
N_{A b v}(T)=\frac{N_{c c}\left(E_{y}, T\right)}{E_{y} \eta Q(T)}
$$

determining the absolute scintillation yield requires measuring the number of charge carriers created in the photodetector, $N_{c c}\left(E_{\gamma} T\right)$. To obtein $N_{\infty c}\left(E_{r} T\right)$ for a $P D$, the umplifier output pulse height is usually calibrated to the charge created in the PD by the direct interaction method or the square wave method, as discussed in Sections 2.2.1 and 2.2.2. respectively. However, an accurate charge calibration for Csl(TI) requires using a method that accounts for the ballistic deficit like the shaped square wave (SSW) method, as discussed in Section 2.2.3. The SSW method is a pulse mode method that was developed for the specific purpose of calibrating amplifier pulse height to the number of electron-hole pairs created in the $P D\left(N_{c e}\left(E_{\gamma} T\right)\right)$ at any temperature, by accouniting for ballistic deficit. If the gamma-ray energy, light collection efficiency, and wavelength-averaged quantum efficiency are known 
at the temperature at which $\mathrm{N}_{\mathrm{ce}}\left(\mathrm{E}_{\boldsymbol{\gamma}} \mathrm{T}\right)$ is measured, then the absolute scintillation yield can he calculated by Eqn. (2.11).

The temperature dependence of the CsI(TI) scintillation yield can be determined using a method that accounts for the ballistic deficit (like the SSW method), or by a method that is unaffected by long decay times. Consequently, an independent, current mode (CM) method that is unaffected by long decay times was also used to measure the relative scintillation yield temperature dependence. The resulting relative yield data was normalized to the room temperature absolute scintillation yield that was determined by the SSW method.

\section{1 $N_{\text {ee }}\left(E_{r} T\right)$ an Determined by Shaped Square Wave (SSW) Method}

\subsubsection{SSW Circult and Method Confirmation}

An analog circuit was designed to implement the SSW method described in Section 2.2.3. The circuit diagram is shown in Fig. 4.1. A square wave with repetition period much greater than both the longest umplifier shaping time constant and greater than any of the CsI(TI) decay times is applied to the input GEN. This signal is split into three RC integration stages. The first two stages simulate the two primary decay time constants of CsI(TI). Each stage consists of a potentiometer (PI,P2), a resistor (RI,R2), a capacitor (C1,C2), and a follower $(A \mid, A 2)$. The time constants are $\tau_{C l}=(P|+R|) C l$ and $\tau_{\mathrm{C} 2}=(\mathbf{P 2}+\mathrm{R} 2) \mathrm{C} 2$. The third branch, with a time constant of $\tau_{\mathrm{Cr}}=(\mathrm{R} 3 \| \mathrm{R} 4) \mathrm{C} 3$, simulates the rise time constant of CsI(TI). The polarity of the rise component signal is opposite of the first two branches and is achieved with an inverting amplifier, A3. Subsequently, the signals from A1, A2, and A3 are introduced to an analog summer, realized by operational amplitier $A 4$. The signals from $A 2$ and $A 3$ are weighted relative to the signal from $A 1$. The weight of the signal from $A 2$, as determined by $k_{1}=Q_{2} / Q_{1}$, is set hy potentiometer $P 3$ 


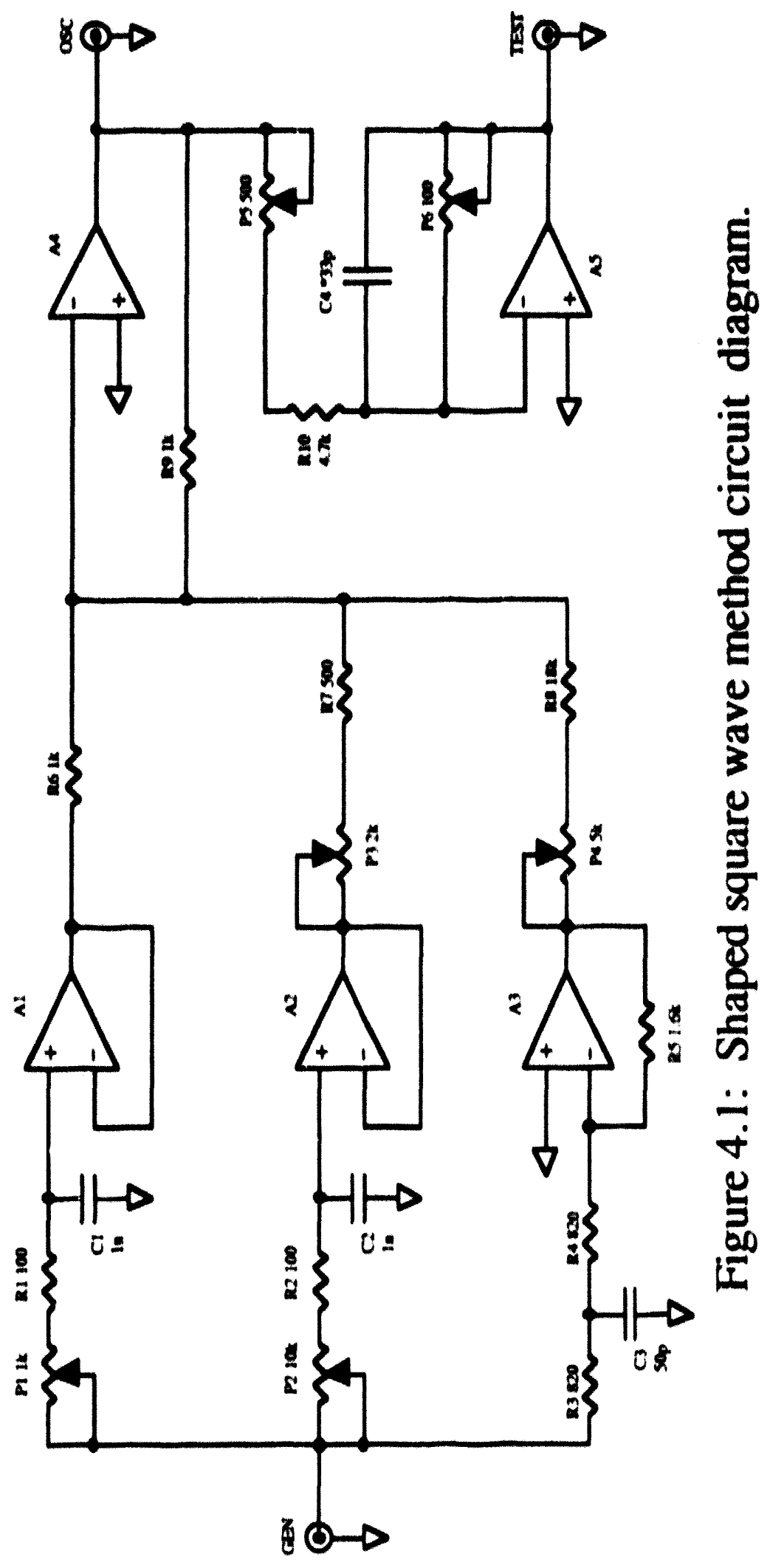


and the resistor $\mathrm{R} 7$. Similarly, $\mathrm{k}_{2}=\tau_{\text {rise }} / \tau_{1}$ is used to weight the signal from $\mathrm{A} 3$ and is set by P4 and R8. The output voltage of the analog summer, A4, represents the simulated pulse shape of Eqn. (2.18).

The calibration signal is then applied to the input of an active divider, realized by operational amplifier A5 (amplifiers A1-A5 are AD847's). The divider allows the pulse amplitude, $\mathrm{V}_{\mathrm{OSC}}$, to be accurately measured by an oscilloscope at the OSC output and then scaled to the desired calibration pulse amplitude at TEST, according to Eqn. (2.20). The ratio of the divider was set to be 50:1 at the maximum value of potentiometer P6. Thus, the preamplifier input voltage pulse amplitude is calculated by

$$
\mathrm{V}_{0}\left(1+\mathrm{k}_{1}-\mathrm{k}_{2}\right)=\frac{\mathrm{V}_{\mathrm{OSC}} \mathrm{S}_{\mathrm{P} 5}}{500}
$$

where $S_{\mathrm{P} 5}$ is the setting of potentiometer P5 (ranging from 0.00 to 10.00 ), such that the charge injected into the preamplifier is

$$
\mathrm{q}_{\mathrm{SSW}}=\mathrm{C}_{\mathrm{eq}} \mathrm{V}_{0}(1+\mathrm{k} 1-\mathrm{k} 2)=\frac{\mathrm{C}_{\mathrm{eq}} \mathrm{V}_{\mathrm{OSC}} \mathrm{S}_{\mathrm{p} 5}}{500}
$$

according to Eqn. (2.20). When the shape and amplitude of the full energy CsI(Tl) scintillation pulse and the SSW calibration pulse after being applied to $\mathrm{C}_{\mathrm{eq}}$ are equivalent (Eqns. (2.17) and (2.21) equivalent), the CsI(Tl) photopeak centroid and the SSW calibration pulse peak centroid will occur in the same channel. If the SSW method has been successfully applied, the calibration peak centroid will match the photopeak centroid as a function of amplifier shaping time.

To confirm the applicability of the SSW method and the implementation of the circuit in Fig. 4.1, a $1 \times 1 \mathrm{~cm}$ right cylindrical CsI(Tl) crystal was mounted on a $1 \times 1 \mathrm{~cm}$ square silicon PIN PD. A charge-sensitive preamplifier, a linear amplifier, and a multichannel analyzer were used to process the scintillation and calibration pulses. Fig. 4.2 demonstrates the behavior described in Sections 2.2, 2.2.1, 2.2.2, and 2.2.3 by showing the photopeak centroid for $\mathrm{CsI}(\mathrm{Tl})$ scintillation events and the calibration pulse peak centroid for the direct interaction (DI), square wave (SW), and SSW methods as a function 


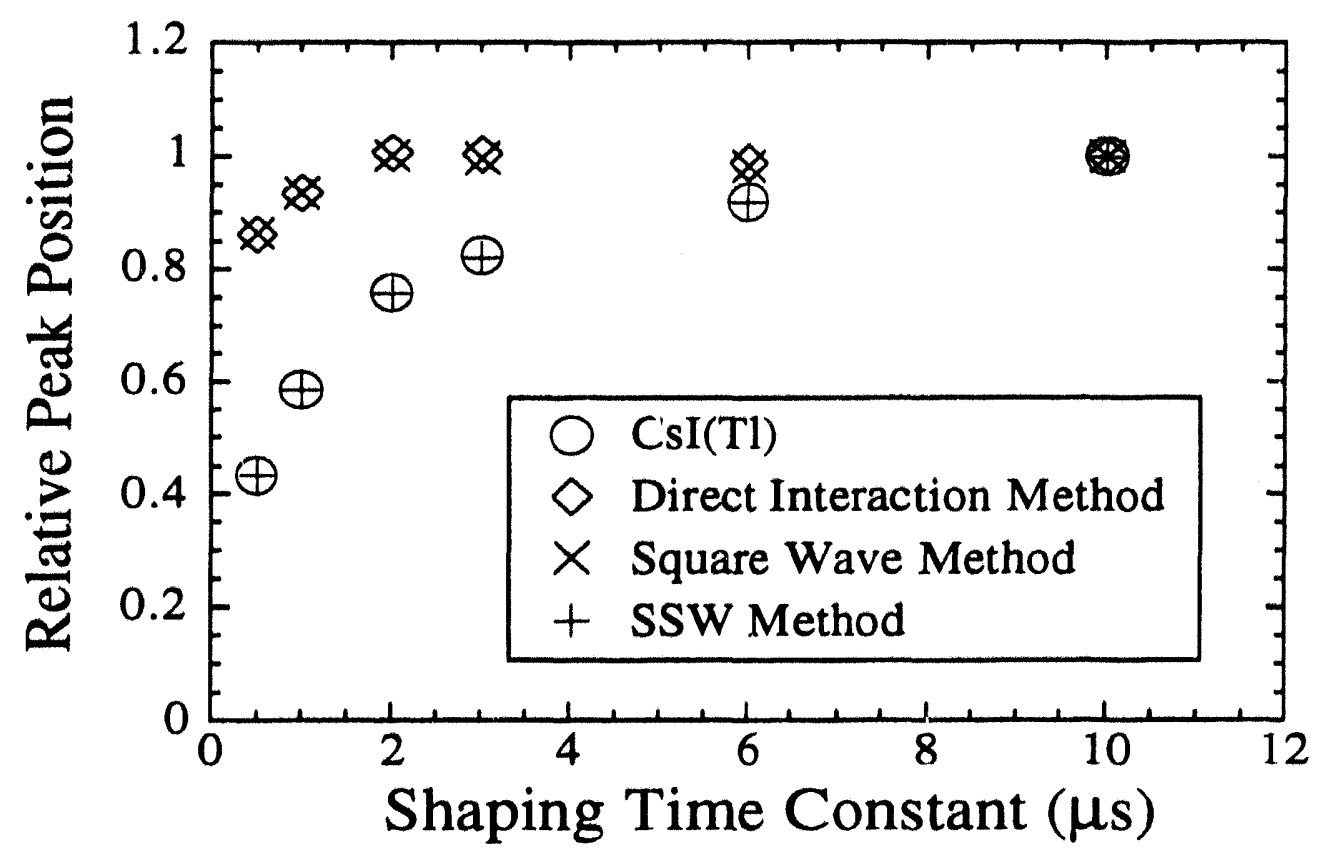

Figure 4.2: Normalized CsI(TI) photopeak and charge calibration peak centroid positions as a function of amplifier shaping time constant.

of amplifier shaping time. The data in Fig. 4.2 are all normalized to unity at the $10 \mu \mathrm{s}$ photopeak centroid to illustrate the increasing effect of ballistic deficit with decreasing shaping time. As previously mentioned, the CsI(Tl) photopeak centroid increases with increasing amplifier shaping time and the SSW method results mimic the CsI(Tl) behavior, while the DI and SW method results are relatively constant with shaping time. To further illustrate the behavior of the calibration techniques, the calibrated number of e-h pairs created in the PD per unit energy deposited $\left(\mathrm{N}_{c c}\left(E_{\gamma}, T\right) / E_{\gamma}\right)$, as a function of amplifier shaping time, has been tabulated in Table 4.1 for each of the three methods. The calibration of amplifier output pulse amplitude to charge by the SSW method results in a shaping time independent calibration, while both the DI and SW methods result in shaping time dependent calibrations. The DI and SW method calibrations show a pulse height degradation of at least $7 \%$ at $10 \mu$ s and are increasingly affected by ballistic deficit with decreasing shaping time. From Fig. 4.2 and Table 4.1, we can conclude that ballistic deficit is a significant problem when calibrating $\operatorname{CsI}(\mathrm{Tl}) / \mathrm{PD}$ systems to charge at room 
Table 4.1: Comparison of charge calibration methods.

\begin{tabular}{|c|c|c|c|}
\hline $\begin{array}{c}\text { Amplifier Shaping } \\
\text { Time Constant } \\
(\mu \mathrm{s})\end{array}$ & \multicolumn{3}{|c|}{ Calibrated Charge (e-h pairs/MeV) } \\
\cline { 2 - 4 } & $\begin{array}{c}\text { Direct Interaction } \\
\text { Method }\end{array}$ & $\begin{array}{c}\text { Square Wave } \\
\text { Method }\end{array}$ & $\begin{array}{c}\text { Shaped Square } \\
\text { Wave Method }\end{array}$ \\
\hline 0.5 & 22,400 & 22,200 & 48,000 \\
\hline 1 & 27,600 & 27,700 & 48,000 \\
\hline 2 & 33,400 & 33,400 & 48,000 \\
\hline 3 & 36,700 & 36,500 & 48,000 \\
\hline 6 & 41,300 & 41,500 & 48,000 \\
\hline 10 & 44,200 & 44,600 & 48,000 \\
\hline
\end{tabular}

temperature. These ballistic deficit problems will become magnified with decreasing temperature as the decay times of CSI(Tl) increase. Consequently, only the SSW method yields an accurate charge calibration for $\mathrm{CsI}(\mathrm{TI}) / \mathrm{PD}$ systems and will thus be the preferred method for absolute scintillation yield of $\mathrm{CsI}(\mathrm{Tl})$ determinations.

\subsubsection{SSW Method Experimental Methods}

The same Solon Technologies, Inc. (STI) $8 \times 8 \times 8 \mathrm{~mm}$ cubic CsI(Tl) crystal that was used for measuring the emission spectrum temperature dependence (Chapter III) was used to measure the temperature dependence of $\mathrm{N}_{c c}\left(\mathrm{E}_{\gamma} \mathrm{T}\right)$. The crystal was wrapped with about twenty layers of white PTFE tape on five sides, while the sixth side was coupled to a $1 \times 1 \mathrm{~cm}$ square Hamamatsu S3590-03 PD via Viscasil 60,000 silicone oil, as seen in Fig. 4.3. The CsI(Tl)/PD combination was mounted on an aluminum box for stability and reproducibility of geometry. To complete the Faraday cage, aluminum rings and a thin aluminum top were mounted on the box, as seen in Fig. 4.4. The aluminum box with the detector mounted on it was placed inside a Tenney Jr. environmental chamber which was modified for liquid nitrogen operation. Initially, the programmable temperature controller on the environmental chamber regulated the flow of liquid nitrogen through a solenoid valve when operating below room temperature, while a heating coil was used when operating above room temperature. However, the turning on and off of the solenoid valve 


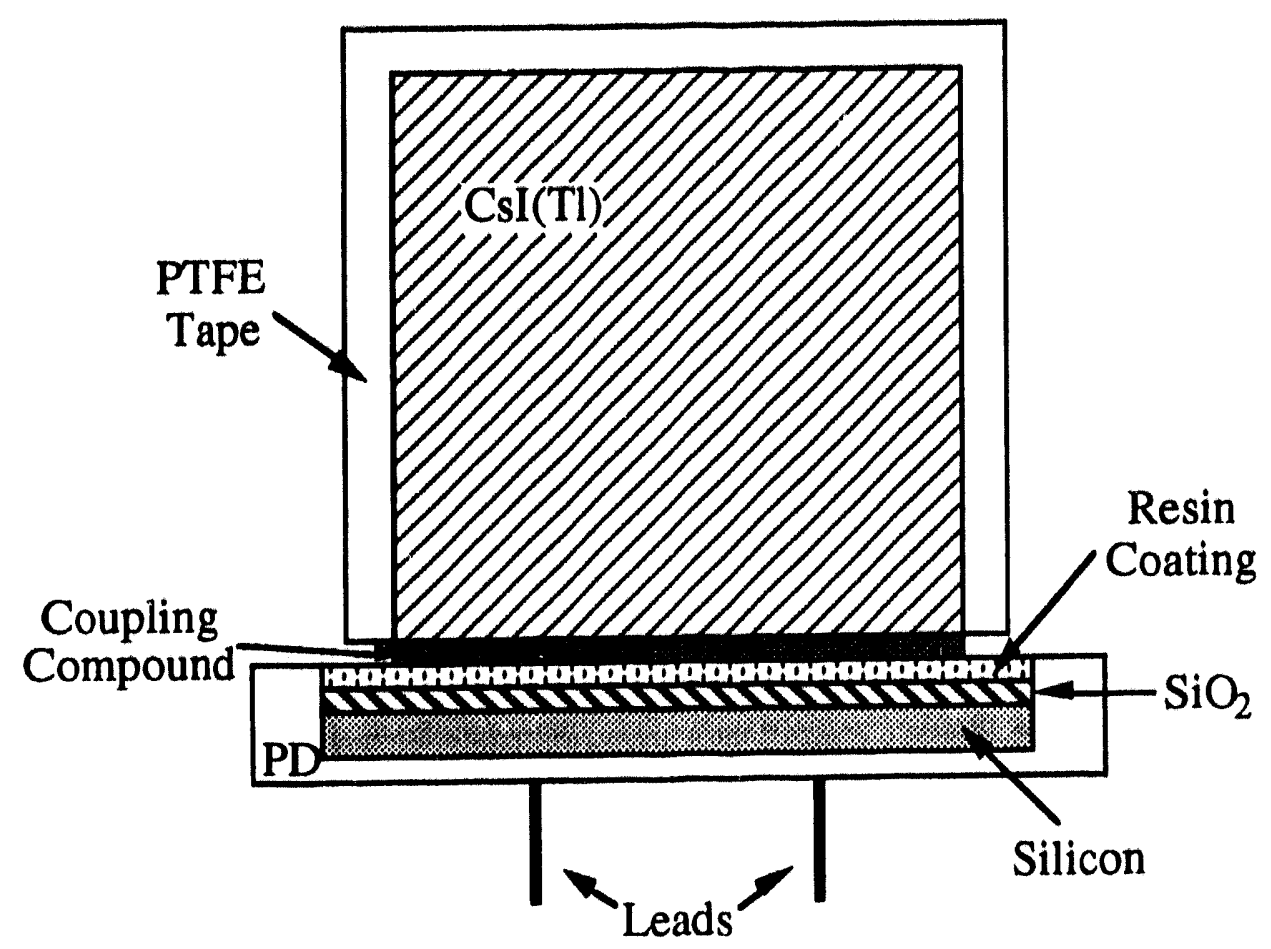

Figure 4.3: $\mathrm{CsI}(\mathrm{Tl})$ crystal and silicon PIN photodiode schematic.

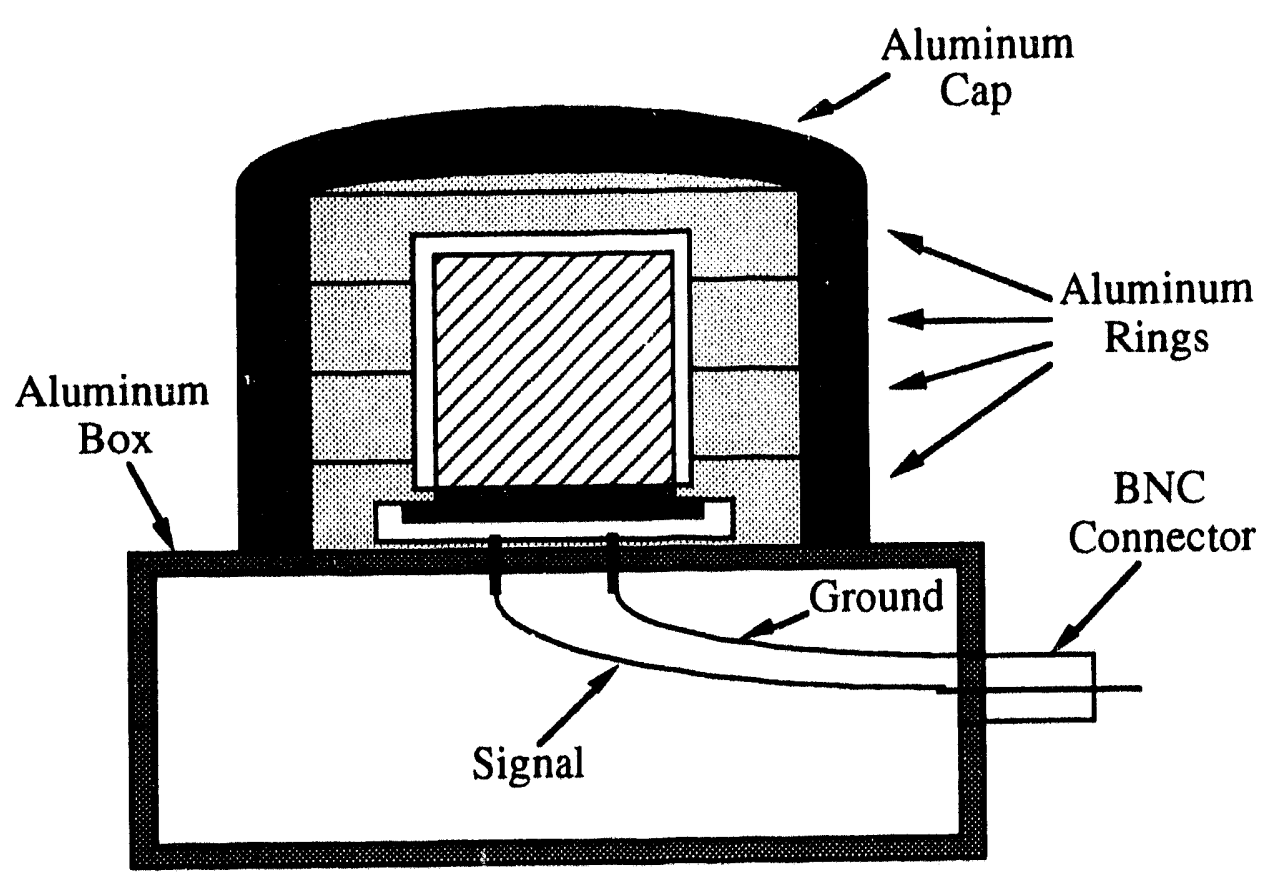

Figure 4.4: Configuration to complete Faraday cage around CsI(Tl)/photodiode detector. 


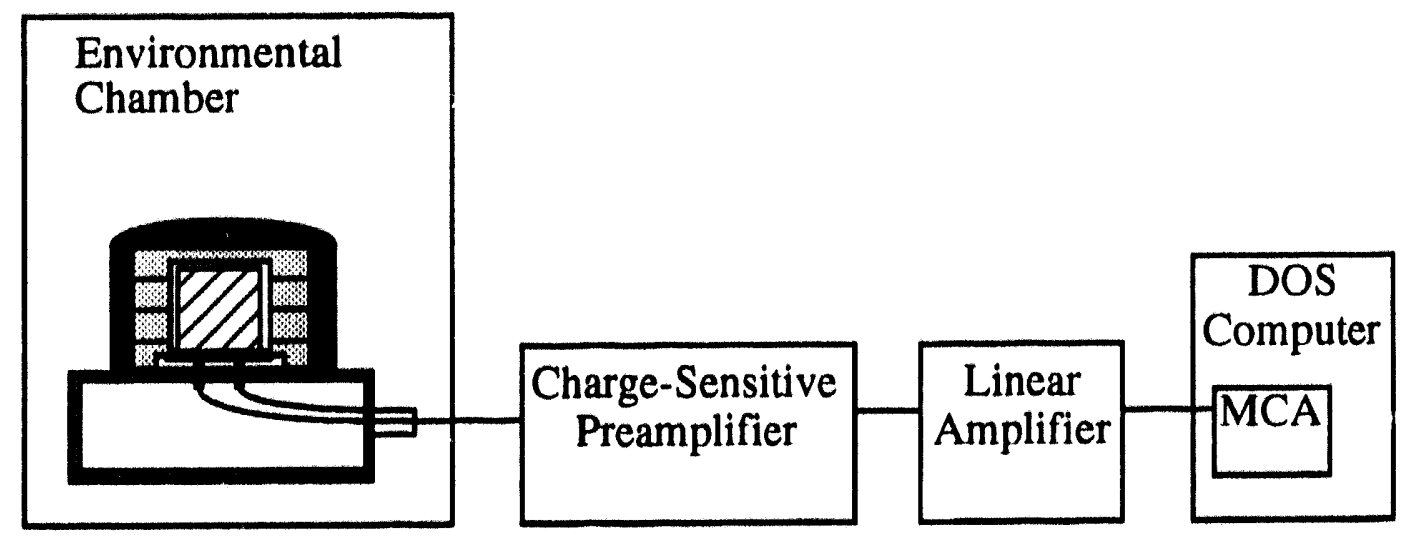

Figure 4.5: $\mathrm{CsI}(\mathrm{Tl}) /$ photodiode electronics schematic.

every second or so was picked up by the PD as noise and resulted in a significant degradation of the system energy resolution. Consequently, the solenoid valve was replaced by a manual valve that was left partially open during operation below room temperature to allow a constant flow of liquid nitrogen into the chamber. The programmable controller then regulated the temperature with the heating coil both above and below room temperature (temperature regulation $\operatorname{method} B$ ).

As seen in Fig. 4.5, the remaining data acquisition electronics consisted of a Canberra 2003BT charge sensitive preamplifier, a Canberra 2021 linear amplifier, a high voltage power supply, a research pulser, and a multi-channel analyzer board. The pulser was used to monitor system drift and not for charge calibration. A short cable connected the PD and preamplifier through a chamber penetration and thus served as a thermal break so that the preamplifier could remain at room temperature while the detector temperature was varied. Two J-type thermocouples, in reproducible positions, monitored the internal temperature near the detector. The uncertainty in the detector temperature at thermal equilibrium was estimated to be $\pm 0.5^{\circ} \mathrm{C}$. Since the Faraday cage for the CsI(Tl) crystal and $\mathrm{PD}$ was a sealed configuration, the detector temperature lagged the temperature outside the Faraday cage during temperature changes. To account for this thermal lag, a temperature run was made with one thermocouple in contact with the detector while another 
thermocouple was in its reproducible position outside the Faraday cage. It was found that the detector temperature came to thermal equilibrium with the exterior about twenty minutes after the outside temperature stabilized when the temperature was increased in $10^{\circ} \mathrm{C}$ increments over the range of -100 to $+50^{\circ} \mathrm{C}$. The time to thermal equilibrium was about twice as long when decreasing the temperature in $10^{\circ} \mathrm{C}$ increments over the same range. Consequently, temperature runs were always begun by cooling the detector to the coldest temperature to be studied and then increased to subsequent temperatures. To assure thermal equilibrium, data acquisition was not begun until at least one hour after the temperature outside the Faraday cage was stabilized.

With the CsI(T1)/PD detector inside the environmental chamber, the temperature was varied over the range of -100 to $+50^{\circ} \mathrm{C}$ in $10^{\circ} \mathrm{C}$ increments. At each temperature, the programmable controller brought the chanber to thermal equilibrium and maintained that temperature throughout data acquisition. Pulse height spectra were acquired at ten of the twelve available linear amplifier shaping time constants $(0.25,0.5,1,2,4,5,6,8,10$, and $12 \mu \mathrm{s})$ at each temperature. The other two shaping times, 1.5 and $3 \mu \mathrm{s}$, were observed to have excessive over-shoot when the pole-zero was not adjusted. To use the SSW method, the photopeak data and calibration pulse peak data must be taken under identical conditions. Since the SSW method is performed after data acquisition, adjusting the pole-zero betwean each spectrum would have made it impossible to apply the SSW method. Consequently, the 1.5 and $3 \mu$ s shaping times were avoided when acquiring the pulse height data and analyzing the data with the SSW method. ${ }^{54} \mathrm{Mn}$ was chosen as the gamma-ray source for its "clean" spectra resulting from a single emission line at $835 \mathrm{keV}$. The peak centroids were determined by taking the first moment of the background subtracted region-of-interest data. The uncertainty in determining the centroid by calculation of the first moment has been derived in Appendix $A$ and was found to be $\leq 1 \%$ for all photopeak and calibration peak centroids [VAL92b]. 


\subsection{3 $N_{c c}\left(E_{\gamma}, T\right)$ Results from SSW Method}

By manually varying the resistances in the SSW circuit of potentiometers P1, P2, and P3 to simulate variations in $\tau_{1}, \tau_{2}$, and $k_{1}$, respectively, the photopeak centroid as a function of amplifier shaping time was replicated using the SSW method at each temperature. It was assumed that variations in the rise time constant with temperature did not have a significant affect on the SSW method due to the small fraction of the total charge contributed by the rise component $\mathrm{k}_{2}$ (about $1 \%$ at room temperature). Thus, both the rise time and the charge contribution were held constant for all temperatures at $\tau_{\text {rise }}=20$ ns and $k_{2}=0.03$. When the calibration pulse centroids match the photopeak centroids at all amplifier shaping times (Section 2.2.3), the number of e-h pairs contributing to the pulse is $N_{c c}\left(E_{\gamma} T\right)=k_{c} q_{s s w}$, where $k_{c}$ is the charge collection efficiency and $q_{s s w}$ is calculated by Eqn. (4.2). Complete charge collection efficiency $\left(k_{c}=1.0\right)$ is approximately realized for a fully depleted PD, thus all e-h pairs that are created in the depletion region will contribute to the signal and $\mathrm{N}_{c c}\left(E_{\gamma} T\right)=q_{s s w}$. The PDs were always reverse biased such that full depletion was achieved. Fig. 4.6 shows some examples of the photopeak and calibration peak centroid matching, while Table 4.2 shows the calibrated e-h pair yield per unit energy deposited, $\mathrm{N}_{c c}\left(\mathrm{E}_{\gamma} \mathrm{T}\right) / \mathrm{E}_{\gamma}$, as a function of temperature. Above $+30^{\circ} \mathrm{C}$, accurate photopeak centroids were not attainable for some amplifier shaping times because of excessive PD noise, while below $-60^{\circ} \mathrm{C}$, the photopeak centroids for the shorter shaping times were obscured by the noise shoulder. The photopeak centroid uncertainty is not considered when matching SSW calibration peak centroids with the photopeak centroids. Thus, any error in a photopeak centroid will either result in an error in the replication of the CsI(TI) pulse shape by the SSW method or in the matching of all photopeak and calibration peak centroids simultaneously not being possible. Similarly, if some of the photopeak centroids can not be resolved, then the shape of the CsI(TI) pulse can not be accurately determined by the SSW method. Additionally, decay time $\tau_{2}$ of $\mathrm{CsI}(\mathrm{Tl})$ hecomes longer than the longest 


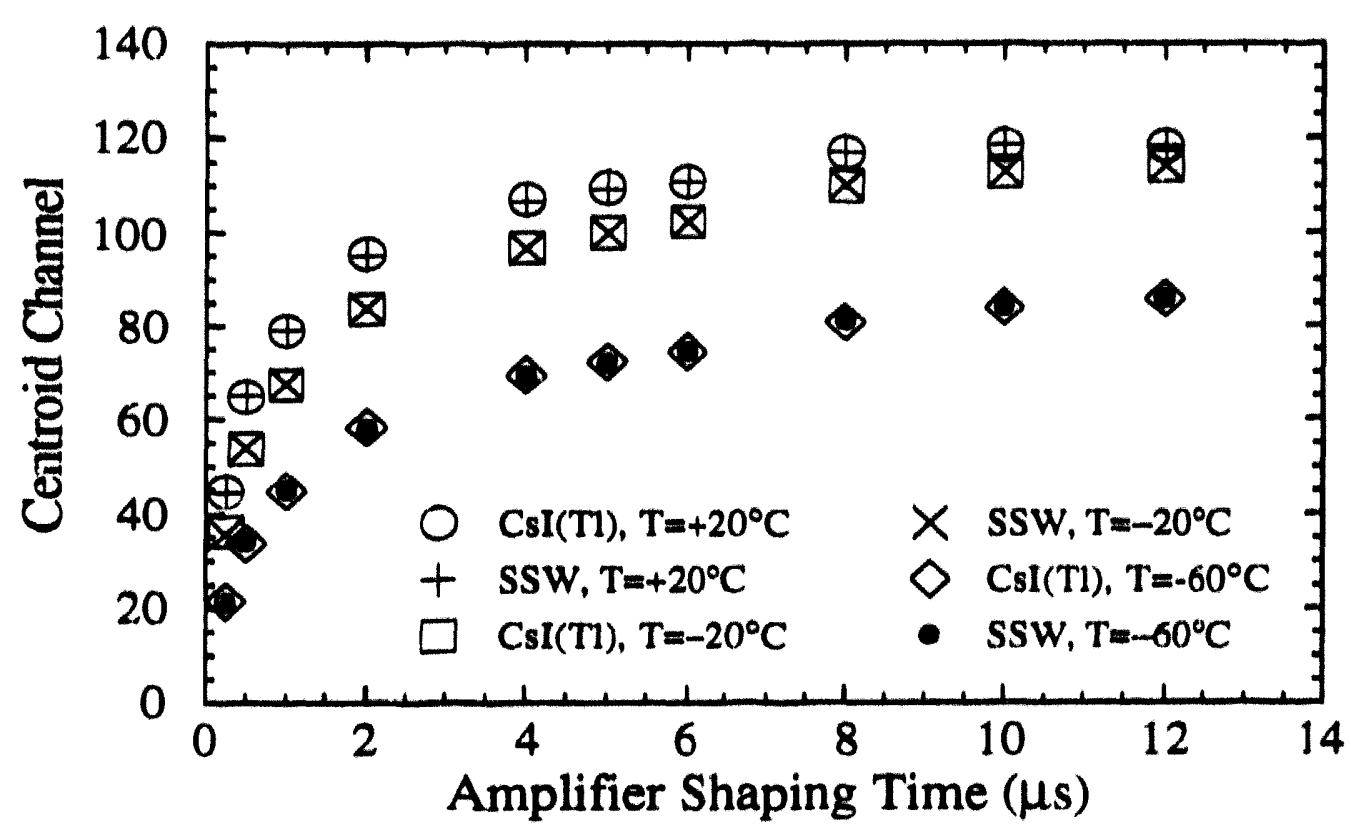

Figure 4.6: Examples of matching CsI(T) photopeak centroids with shaped square wave (SSW) method calibration pulse peak centroids for Solon Technologies, Inc. crystal.

Table 4.2: Temperature dependence of electron-hole pair yield per unit energy deposited as determined by the shaped square wave method for Solon Technologies, Inc. CsI(TI) crystal.

\begin{tabular}{|c|c|}
\hline $\begin{array}{c}\text { Temperature } \\
\left({ }^{\circ} \mathrm{C}\right)\end{array}$ & $\begin{array}{c}\mathrm{N}_{\mathrm{cc}}\left(\mathrm{E}_{\boldsymbol{\gamma}} \mathrm{T}\right) / \mathrm{E}_{\gamma}{ }^{*} \\
(\mathrm{e}-\mathrm{h} \text { pairs } / \mathrm{MeV})\end{array}$ \\
\hline+30 & 47,000 \\
\hline+20 & 47,800 \\
\hline+10 & 49,100 \\
\hline 0 & 49,700 \\
\hline-10 & 49,700 \\
\hline-20 & 49,200 \\
\hline-30 & 48,600 \\
\hline-40 & 45,600 \\
\hline-50 & 41,100 \\
\hline-60 & 39,800 \\
\hline
\end{tabular}

$\pm 3 \%$ estimated uncertainty 
amplifier shaping time below $-60^{\circ} \mathrm{C}$. As discussed in Section 2.2.3, the SSW method is only sensitive to decay times that are similar to the amplifier shaping times. Consequently, determining $\mathrm{N}_{\mathrm{ce}}\left(\mathrm{E}_{\gamma}, \mathrm{T}\right)$ for $\mathrm{T}>30^{\circ} \mathrm{C}$ and $\mathrm{T}<-60^{\circ} \mathrm{C}$ by the $\mathrm{SSW}$ method was not possible. The e-h pair yield at $-10^{\circ} \mathrm{C}$ and $0^{\circ} \mathrm{C}$ of 49,700 per $\mathrm{MeV}$ suggests that the maximum occurs around $-5^{\circ} \mathrm{C} . \mathrm{N}_{\mathrm{cc}}\left(\mathrm{E}_{\gamma} \mathrm{T}\right) / \mathrm{E}_{\gamma}$ drops to about $80 \%$ of the -10 and $0{ }^{\circ} \mathrm{C}$ yield at $-60^{\circ} \mathrm{C}$, and about $94.5 \%$ at $+30^{\circ} \mathrm{C}$.

The uncertainty in $\mathrm{N}_{\mathrm{cc}}\left(\mathrm{E}_{\gamma} \mathrm{T}\right) / \mathrm{E}_{\gamma}$ is dominated by the uncertainty associated with the SSW method, since the error in peak centroid calculations was small ( $51.0 \%)$. The uncertainty in determining the number of e-h pairs created in the PD hy the SSW method is dominated by the uncertainty of the measurement of $V_{\text {OsC }}$ (estimated to be $2.5 \%$ ) and the precision of $C_{\text {Teat }}(1.0 \%)$. Therefore, it has heen estimated that each value of $N_{c c}\left(E_{\gamma} T\right)$ has an uncertainty of about $\pm 3 \%$.

To confirm that the CsI(TI) crystals procured from Bicron, Horiba, and Rexon would yield similar $\mathrm{N}_{c c}\left(\mathrm{E}_{\gamma}, \mathrm{T}\right)$ results to those reported for the STI crystal, the photopeak centroids for all of the crystals were recorded at room temperature for all amplifier shaping times and are shown in Fig. 4.7. It is ohserved that the centroids of the Bicron, Horiba, and Rexon crystals are within $8 \%$ of the STI crystal centroids at each shaping time. If we assume that pulse height at $12 \mu \mathrm{s}$ is indicative of $\mathrm{N}_{\mathrm{cc}}\left(\mathrm{E}_{\gamma} \mathrm{T}\right)$ that would be measured using the SSW method, then the expected standard deviation of crystal-to-crystal variations is about $\pm 3.6 \%$. Thus, the expected variation in the measured $N_{c c}\left(E_{\gamma}, T\right)$ results by the $S S W$ method from crystal to crystal is less than $\pm 5 \%$, the square root of the quadrature sum of the SSW uncertainty (3\%) and the observed crystal-to-crystal centroid variations (3.6\%).

\subsection{Light Collection Effiency, $\eta$}

The light collection efficiency, $\eta$, for the geometric and optical properties of the $\mathrm{CsI}(\mathrm{Tl}) / \mathrm{PD}$ detector were modeled using the visible light transport program DETECT 


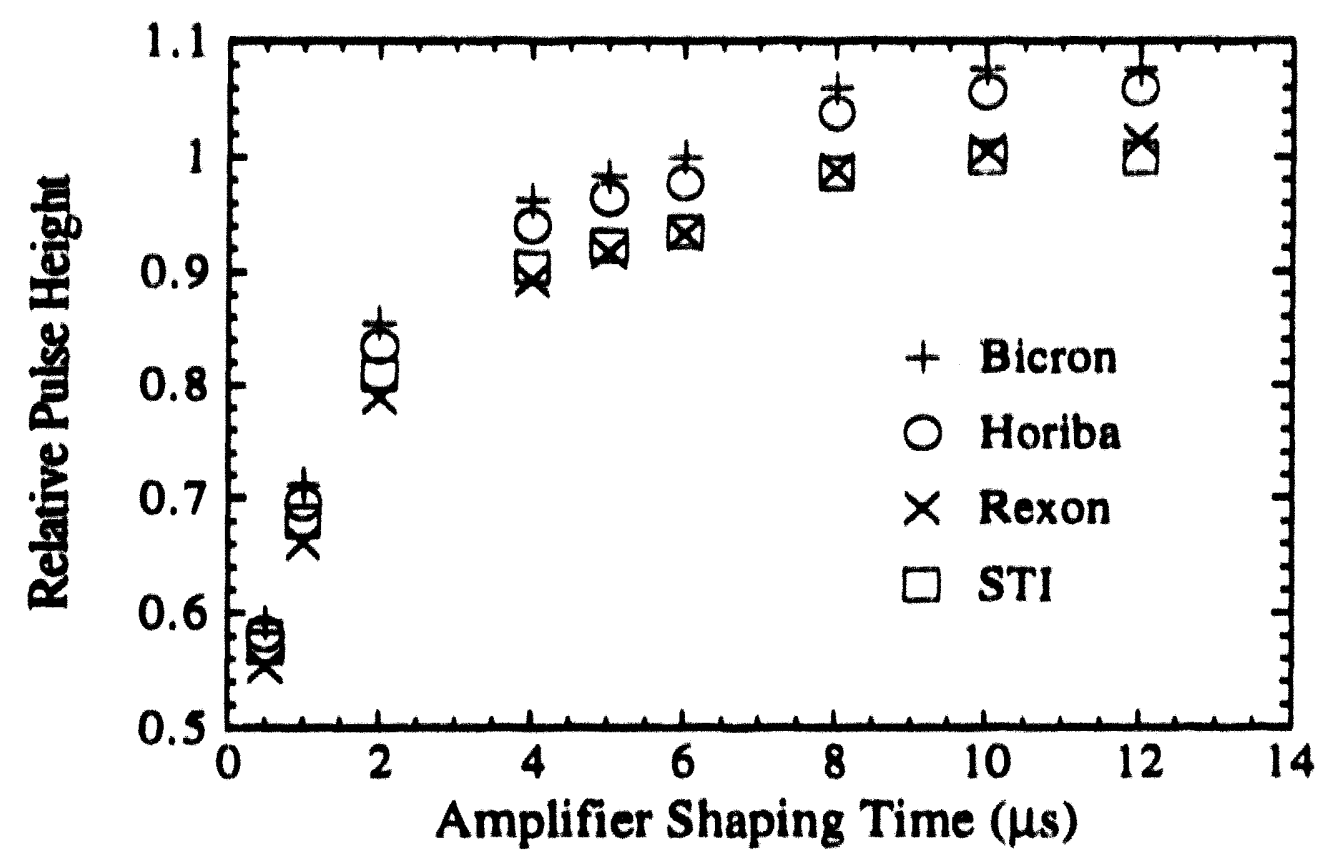

Figure 4.7: Amplifier shaping dime dependence of CsI(TI) pulse height for crystals from Bicron Corporation, Horiba Crystal Products, Rexon Components, Inc., and Solon Technologies, Inc. Normalized to the $12 \mu \mathrm{s}$ pulse height of the STI crystal.

[KNO88]. Using our definition of $\eta$ (the probability that a scintillation photon is absorbed by the PD) required that DETECT be modified to account for the Fresnel reflection of photons from the PD back into the scintillator. The modification first required the calculation of the photon transmission angle using Snell's law:

$$
\theta_{2}=\sin ^{-1}\left[\sin \left(\theta_{1}\right) \frac{n_{1}}{n_{2}}\right] \text {. }
$$

where $\theta_{1}$ and $\theta_{2}$ are the angles of incidence and transmission, respectively, and $n_{1}$ and $n_{2}$ are the index of refraction for the thin layer $(100 \mathrm{~nm})$ of $\mathrm{SiO}_{2}\left(n_{1}=1.46\right)$ that covers the silicon and the index of refraction for silicon $\left(n_{2}=4.08\right.$ at $\left.550 \mathrm{~nm}\right)$. Subsequently, the probability of reflection for randomly polarized photons is calculated using the Fresnel equations. The probability of reflection for parallel polarized photons is

$$
\rho_{\|}=\frac{\tan ^{2}\left(\theta_{1}-\theta_{2}\right)}{\tan ^{2}\left(\theta_{1}+\theta_{2}\right)} \text {. }
$$

and the probability of reflection for perpendicular polarized photons is 


$$
\rho_{1}=\frac{\sin ^{2}\left(\theta_{1}-\theta_{2}\right)}{\sin ^{2}\left(\theta_{1}+\theta_{2}\right)} .
$$

The probability of reflection for randomly polarized photons (which is what we assume photons that hit the PD are) is the average of the parallel and perpendicular probabilities

$$
\rho_{\text {tand }}=\frac{\rho_{1}+\rho_{1}}{2} \text {. }
$$

Finally, a Monte Carlo calculation is performed to determine whether the photon is reflected or transmitted at the $\mathrm{SiO}_{2}$ to sillicon interface. If the photon is reflected, the program continues to follow its fate, while photons that are transmitted into the silicon ure assumed to be absorhed in the PD. For the geometry and optical properties of the CsI(TI) crystal $(n=1.8)$, coupling compound $(n=1.5)$, and PD used, the light collection efficiency was calculated to be $81.4 \%(\eta=0.814)$. The thin resin coating $(n=1.5)$ that seals the PD from the atmosphere (and is thus in contact with the coupling compound) was also modeled. The surfaces of the scintillator were all polished and modeled accordingly, while the white PTFE tape used to wrap the crystals was assumed to have a reflectance of 0.98 . The input file used and part of the output file produced by DETECT appear in Appendix B.

The index of refraction for silicon is wavelength dependent and therefore causes the modeled light collection efficiency to be dependent on $\lambda$, as seen in Fig. 4.8. The light collection efficiency is observed to decrease with the increasing index of refraction of silicon due 10 the increased prohahility of reflection at the $\mathrm{SiO})_{2}$ to silicon interface. Although $\eta$ varies hy ahout $4 \%$ over the range of the CsI(TI) emission spectrum, most of the variation is observed to occur below $450 \mathrm{~nm}$ where only a small fraction of the Csl(TI) light is emitted (Fig. 3.7). Around the $560 \mathrm{~nm}$ peak emission of CsI(TI), $\eta$ varies hy ahout $\pm 1 \%$; consequently, we have assumed that $\eta$ is independent of $\lambda$.

The value of $\eta$ used from DETECT was determined assuming no bulk absorption or hulk scattering of scintillation photons in Csl(TI). Although this is not actually the case, a realistic hulk ahsorption mean free path is on the order of a few meters [VIE74]. A $5 \mathrm{~m}$ 


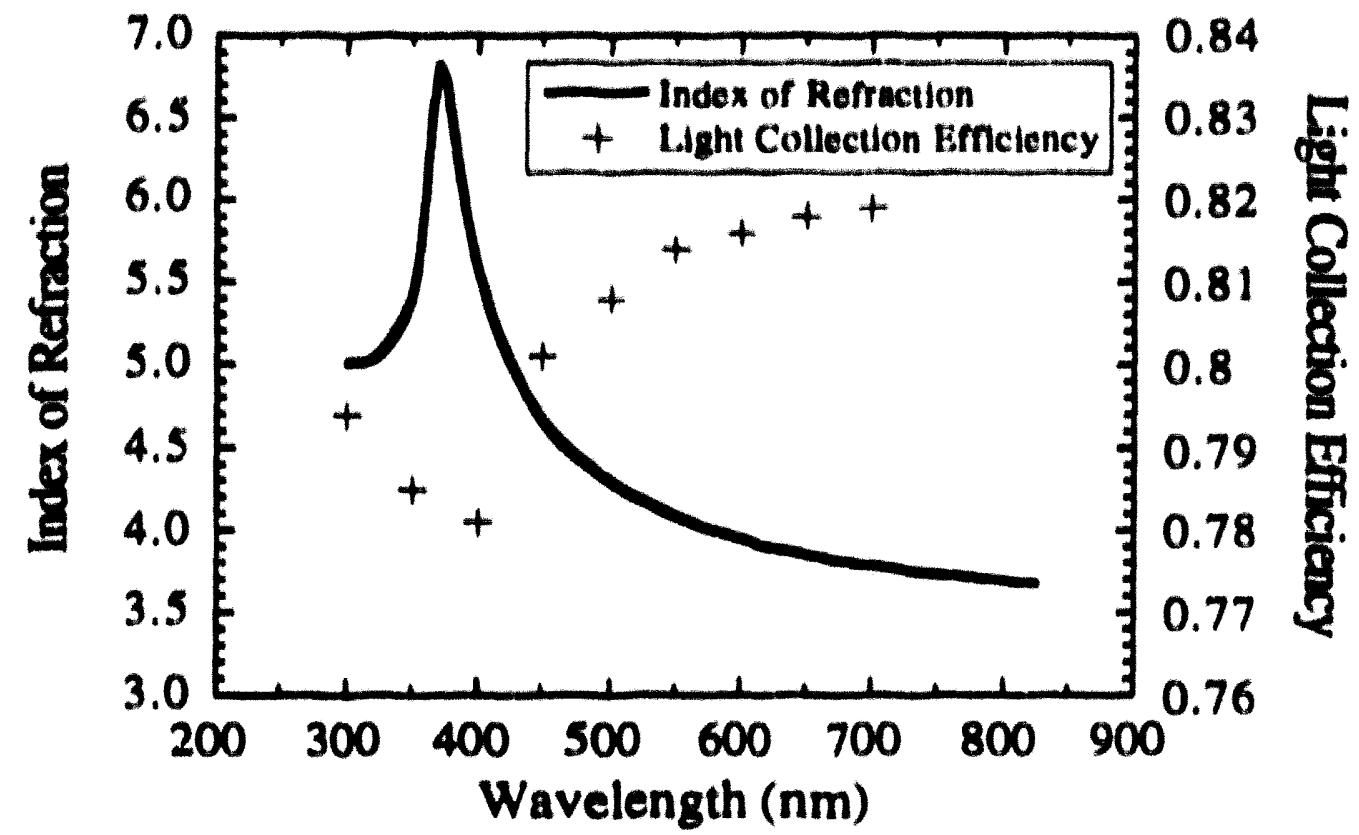

Figure 4.8: Variation of light collection efficiency with scintllation photon wavelength due to the change in the index of refraction for silicon.

mean free path reduced the light collection efficiency by about $1 \%$, while a $1 \mathrm{~m}$ mean free path reduced $\eta$ by about $6 \%$. Introducing bulk scattering into the simulation did not change $\eta$ significantly due to the small dimensions of the crystal.

Considering the variations described, it has been estimated that the uncertainty of the simulated light collection efficiency is $\eta=(1) .814 \pm 0.024$ (or $\pm 3 \%$ relative uncertainty). This result has been confirmed by Bird, et al. [BIR91, BIR92] by an independent Monte Carlo program and experimental measurements. Bird, et al. report a light collection efficiency of about 0.785 from their program and ahout 0.79 from their experimental measurements for a $|x| x \mid \mathrm{cm}$ cubic CsI(TI) crystal coupled to a $|x| \mathrm{cm}$ silicon PIN photodiode. Although our crystal was slightly smaller, we do not believe the difference is significant hecause when their system was modeled with DETECT a value of $\eta=() .817$ was found. Bird, et al. also modeled and measured experimentally the light collection efficiency of crystals with several different lengths. Since the optical properties that most affect the light collection efficiency are surface treatment and reflective wrapping, to henchmark a 
model it is necessary to have goud agreement hetween modeled and measured values for different detector geometries. Differences in geometry result in different average numbers of surface interactions, which result in confirmation of the accuracy of the surface treatiment and reflective wrapping modeling. Thus, if the modeled and experimental light collection efficiency results agrec for different crystal lengths (different geometries), then we can be reasonably confident that the model is accurately resembling the experiment. These results are further discussed in Section 4.5.

\subsection{Wavelength.Averaged Quantum Emciency}

We have defined quantum efficiency, $Q(\lambda, T)$, as the prohahility that a photon of wavelength $\lambda$ that is ahsorthed in the PD will create an $\mathrm{e} \cdot \mathrm{h}$ pair when the PD is heing operated at temperature $T$. We shall also refer $I 0 Q(\lambda, T)$ as the internal quantuin efficiency when we wish to distinguish our definition from the conventional definition of quantum effieiency, which we shall term external quantum efficiency. The wavelength-averaged quantum efficiency $\bar{Q}(T)$ has been defined as the quantum efficiency averaged over the scintillation emission spectrum. $Q(\lambda . T)$ and $\bar{Q}(\mathrm{~T})$ defined in this manner are independent of the geometry and optical properties of the detector. and thus are fundamental characteristics of the PD. II a scintillation photon creates more than one $\mathrm{e}-\mathrm{h}$ pair, as is possible for photons with energies at least twice the hand gap of silicon, the definition of quantum efficiency must be reconsidered. However. Christensen [CHR76] has shown that the prohability of a single photon creating more than one $\mathrm{e}-\mathrm{h}$ pair in silicon is approximately zero for $\lambda \geq 350 \mathrm{~nm}$. Consequently, our definitions of $Q(\lambda, T)$ and $\bar{Q}(T)$ seem to hold rigorously for CsI(TI) coupled to a PD. These definitions may not he well-suited for a photomultiplier tute hecause of the significant transmission of light through the photocathode.

To delermine $\bar{Q}(\mathrm{~T})$ by Eqn. (2.11), hoth $\varepsilon(\lambda, T)$ and $Q(\lambda, T)$ must he known. The emission spectrum of $\mathrm{CsI}(\mathrm{Tl}), \varepsilon(\lambda, \mathrm{T})$, was measured in Chapter III; Fig. 3.7 shows 
$\varepsilon\left(\lambda, 25^{\circ} \mathrm{C}\right)$ for the crystals from Bicron, Horiba, Rexon, and STI and Fig. 3.8 shows $E(\lambda, T)$ for the STI crystal over the range of $-1(x)$ to $+50^{\circ} \mathrm{C}$ in $25^{\circ} \mathrm{C}$ increments. The data in Figs. 3.7 and 3.8 must be normalized to Eqn. (2.5) for Eqn. (2.10) to be valid.

A calihrated Hamamatsu 3590-03 silicon PIN photodiode was procured to facilitate the accurate determination of both $Q(\lambda, T)$ and $\bar{Q}(T)$. The room temperature radiant sensitivity of the PD, $R\left(\lambda, 25^{\circ} \mathrm{C}, \theta\right)$, was calibrated by Hamamatsu using a NIST traceable standard PD over the range of 360 to $4(X 0 \mathrm{~nm}$ in $10 \mathrm{~nm}$ increments and $4(X)$ to $11(X) \mathrm{nm}$ in $20 \mathrm{~nm}$ increments. Appendix $\mathrm{C}$ contains an equipment schematic for the calibration and the resulting radiant sensitivity as a function of wavelength. $R(\lambda, T, \theta)$ is defined as the current induced by an incident photc: : beam of known power at wavelength $\lambda$, temperature $T$, and angle $\theta$, and is measured by recording the current induced hy a known beam of photons that is perpendicularly incident $\left(\theta=-()^{\circ}\right)$ on the PD through air, as seen in Fig. 4.9. To determine the intermal quantum efficiency $Q(\lambda, T)$, the radiant sensitivity is first converted to external quantum efficiency $Q_{0 \times 1}(\lambda, T, \theta)$ and then corrected for Fresnel reflection in the calibration process at each interface, as seen in Eqn. $(2.7), R\left(\lambda .25^{\circ} \mathrm{C}, \theta=()^{\circ}\right)$ is converted to external quantum efficiency hy

$$
Q_{0 \times 1}\left(\lambda .25^{\circ} \mathrm{C}, \theta=0^{\circ}\right)=\frac{h c}{e} \frac{R\left(\lambda, 25^{\circ} \mathrm{C}, \theta=0^{\circ}\right)}{\lambda}=1.24 \frac{R\left(\lambda, 25^{\circ} \mathrm{C}, \theta=0^{\circ}\right)}{\lambda} .
$$

where h is Planck's constant, $\mathrm{c}$ is the velocity of light, and $\mathrm{e}$ is the clectronic charge. When $R\left(\lambda, 25^{\circ} \mathrm{C}, \theta=()^{\circ}\right)$ is expressed in $\mathrm{mA} W$ and $\lambda$ in $\mathrm{nm}$, a value for he/e of 1.24 will correctly cancel units. To correct the calibration for light lost due to index of refraction mismatches, the fraction of perpendicularly incident light that is Fresnel reflected, $\left.\rho(\theta=)^{\circ}\right)$, at an interface when passing from material I to material 2 is calculated by

$$
\rho\left(\theta=\left(0^{\circ}\right)=\left[\frac{n_{1}-n_{2}}{n_{1}+n_{2}}\right]^{2},\right.
$$

where $n_{1}$ and $n_{2}$ are the indices of refraction for materials 1 and 2 , respectively. Substituting values for $n_{1}$ and $n_{2}, 4 \%$ of the incident photons are Fresnel reflected at the air 


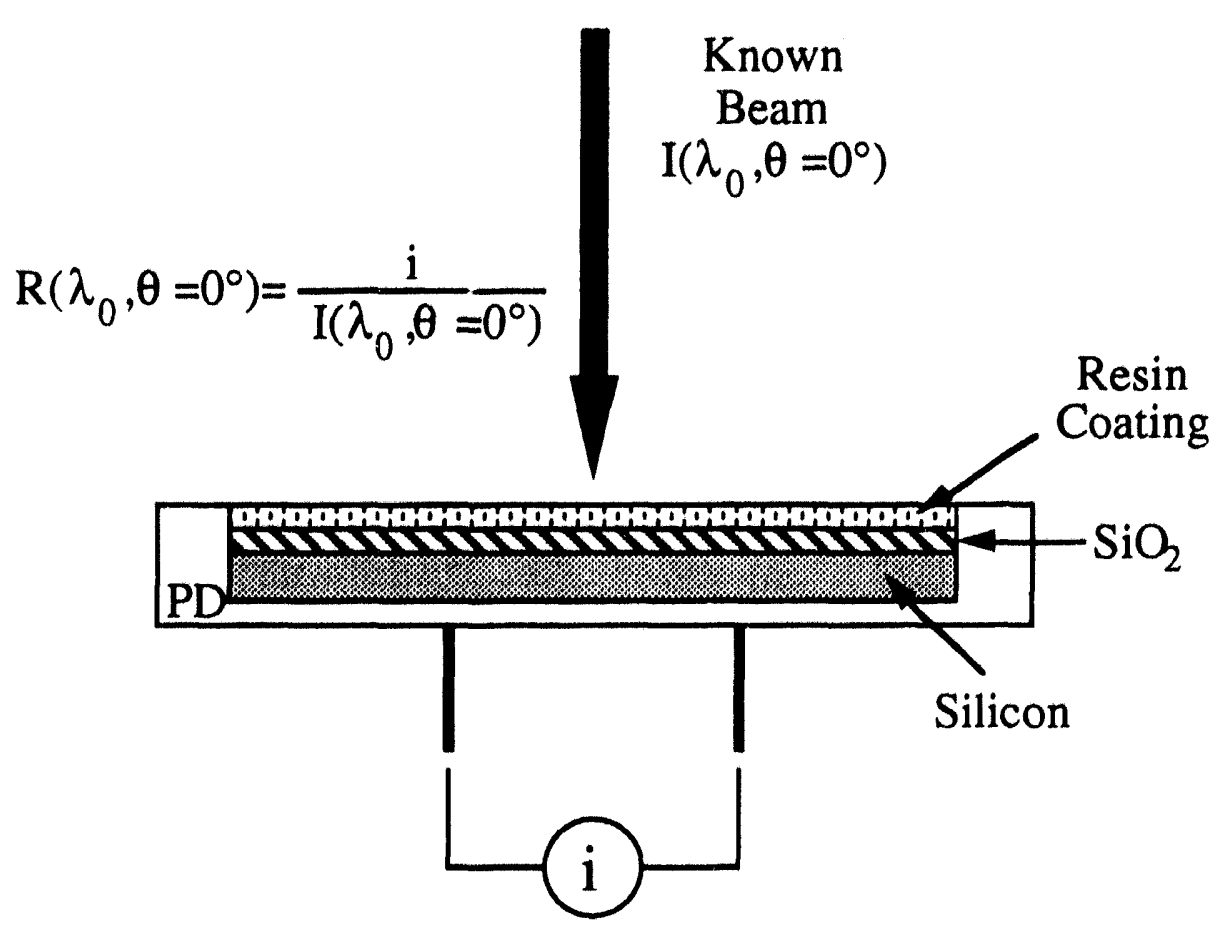

Figure 4.9: Schematic for photodiode calibration.

to resin coating interface and less than $1 \%$ are reflected at the resin coating to $\mathrm{SiO}_{2}$ interface. Since the index of refraction of silicon is wavelength dependent, $\rho$ is also a function of $\lambda$ at the $\mathrm{SiO}_{2}$ to silicon interface. The fraction of reflected photons in the range of the $\operatorname{CsI}(\mathrm{Tl})$ emission spectrum ranges from a maximum of $\rho\left(\theta=0^{\circ}\right)=34.2 \%$ at $\lambda=400 \mathrm{~nm}$ to a minimum of $\rho\left(\theta=0^{\circ}\right)=19.6 \%$ at $\lambda=700 \mathrm{~nm}$ at the $\mathrm{SiO}_{2}$ to silicon interface. Recalling Eqn. (2.8):

$$
\mathrm{Q}(\lambda)=\frac{\mathrm{Q}_{\mathrm{ext}}\left(\lambda, \theta_{0}\right)}{1-\rho\left(\lambda, \theta_{0}\right)}
$$

the angle independent internal quantum efficiency is calculated from the angle dependent external quantum efficiency, and both are shown in Fig. 4.10. The resulting $Q\left(\lambda, 25^{\circ} \mathrm{C}\right)$ for the PD and the previously determined $\varepsilon\left(\lambda, 25^{\circ} \mathrm{C}\right)$ for each $\mathrm{CsI}(\mathrm{Tl})$ crystal normalized to Eqn. (2.5) are then used to evaluate $\overline{\mathrm{Q}}\left(25^{\circ} \mathrm{C}\right)$ in Eqn. (2.10) numerically using Simpson's method. The results appear in Table 3.1 for each crystal.

The temperature dependence of $Q(\lambda, T)$ was determined by measuring the 


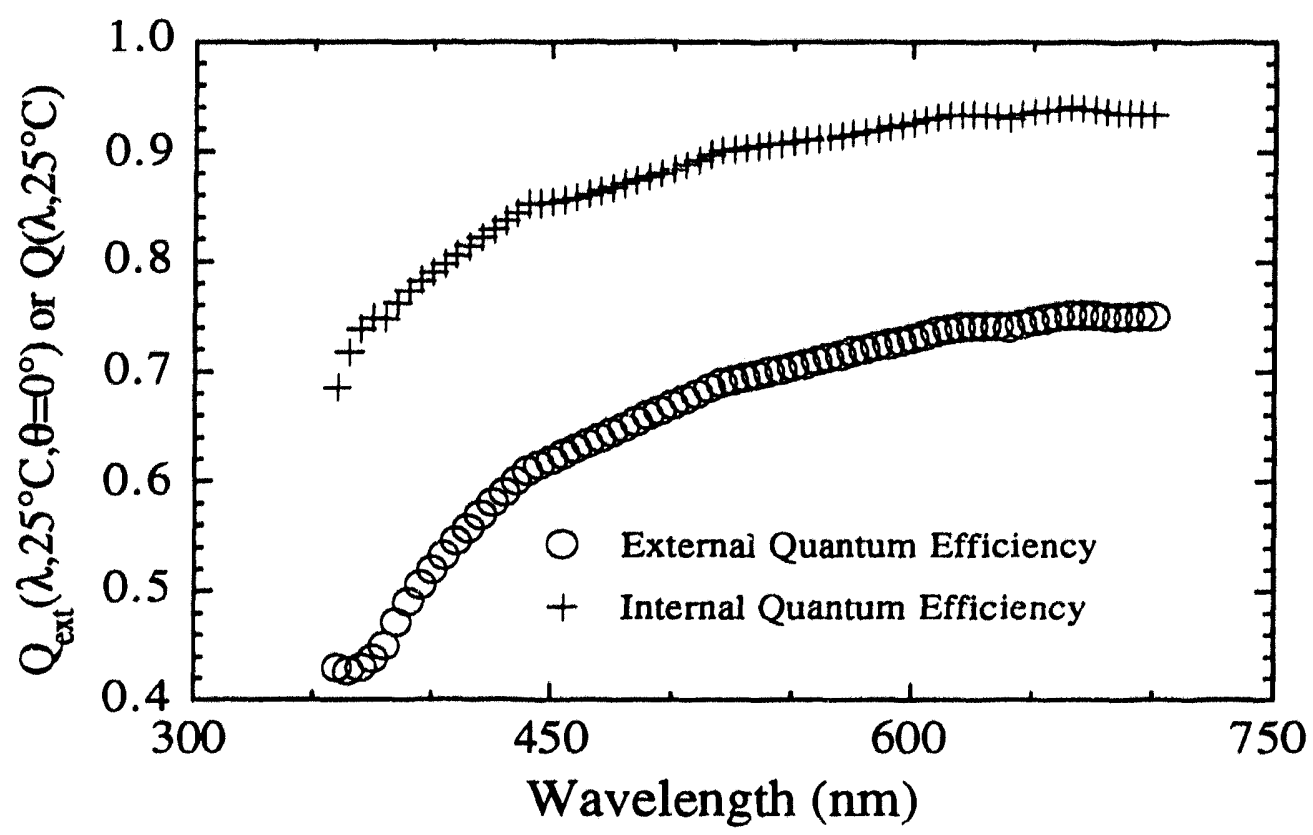

Figure 4.10: External and internal quantum efficiency of calibrated Hamamatsu S3590-03 silicon PIN photodiode.

temperature dependence of the PD pulse height response to a green light emitting diode (LED). For the LED used, Eqn. (2.1) is generalized to

$$
\mathrm{N}_{c c}(\mathrm{~T})=\int_{0}^{\infty} \int_{0}^{\infty} \mathrm{N}_{\mathrm{LED}}(\lambda, \mathrm{T}, \mathrm{t}) \eta_{\mathrm{LED}}(\lambda, \mathrm{T}, \mathrm{t}) \mathrm{Q}(\lambda, \mathrm{T}, \mathrm{t}) \mathrm{d} \lambda \mathrm{dt},
$$

where $N_{L E D}$ is the number of photons emitted by the LED, $\eta_{\text {LED }}$ is the light collection efficiency of the LED and PD, and $Q$ is the PD quantum efficiency as functions of wavelength $\lambda$, temperature $T$, and time $t$. The narrow emission bandwidth of the LED around $\lambda_{0}$ can be assumed to be a delta function, $\delta\left(\lambda-\lambda_{0}\right)$. If the LED is flashed for a very short time and kept at room temperature, while the temperature of the PD is varied, then $\mathrm{N}_{\text {LED }}(\lambda, T, t)=\mathrm{N}_{0} \delta\left(\lambda-\lambda_{0}\right) \delta(t)$, where $\mathrm{N}_{0}$ is the number of photons emitted each time the LED is pulsed. Again, assuming that $\eta$ is independent of time, temperature, and wavelength and that $Q$ is independent of time, Eqn. (4.8) becomes

$$
\mathrm{N}_{c c}(T)=\mathrm{N}_{0} \eta_{\mathrm{LED}} \mathrm{Q}\left(\lambda_{0}, \mathrm{~T}\right)=\mathrm{kQ}\left(\lambda_{0}, \mathrm{~T}\right),
$$


where $\mathrm{k}$ is a constant. This implies that the temperature dependence of $\mathrm{N}_{c c}(T)$ for the LED depends only on the temperature dependence of the quantum efficiency. Since the LED is pulsed for a very short time, the pulse processing of the LED pulses 'vill be similar to that of the direct interaction and square wave methods of charge calibration (Sections 2.2.1 and 2.2.2) and no ballistic deficit will be observed. Consequently, the amplifier output pulse will be proportional to $\mathrm{N}_{c c}(T)$ and thus proportional to the PD quantum efficiency.

Flashing the green LED though a window in the environmental chamber allowed the LED temperature to be maintained at about $+23^{\circ} \mathrm{C}$, while the $\mathrm{PD}$ temperature was varied over the range of -100 to $+50^{\circ} \mathrm{C}$. The $\mathrm{CsI}(\mathrm{Tl})$ crystal was not mounted on the PD and the aluminum cap that completed the Faraday cage was replaced by a sheet of aluminum foil with a small hole in it. Noise levels were observed to increase because of the hole in the Faraday cage, but the pulser peak from the LED was still easily resolved. The LED measurements resulted in a change in pulse amplitude of less than $1 \%$ from $+30^{\circ} \mathrm{C}$ to $-60^{\circ} \mathrm{C}$, thus $\mathrm{Q}\left(\lambda_{0}, \mathrm{~T}\right)=\mathrm{Q}\left(\lambda_{0}\right)$. It was then assumed that this temperature independence of the quantum efficiency at $\lambda_{0}$ also holds for the entire emission spectrum of $\mathrm{CsI}(\mathrm{Tl})$ so that $\mathrm{Q}(\lambda, \mathrm{T})=\mathrm{Q}(\lambda)$. Consequently, the values tabulated in Table 3.2 for the emission spectrum of the Solon Technologies, Inc. CsI(Tl) crystal are the best estimates of the $\bar{Q}(T)$. Since the SSW method could only be applied between -60 and $+30^{\circ} \mathrm{C}$, the quantum efficiency results are of primary interest in the same temperature range. From Table 3.2, we see that $\bar{Q}(T)$ varies by about $1 \%$ between -75 and $+50^{\circ} \mathrm{C}$. Since we have estimated the uncertainty of determining $\overline{\mathrm{Q}}\left(25^{\circ} \mathrm{C}\right)$ to be \pm 0.018 (or $\pm 2.0 \%$ relative unccrtainty), any variation of $\bar{Q}(\mathrm{~T})$ with temperature is within the room temperature uncertainty. Furthermore, assuming that the wavelength-averaged quantum efficiency is independent of temperature $(\bar{Q}(T)=\bar{Q}=0.897)$ will not introduce any significant uncertainty. 


\subsection{Relative Scintillation Yield Temperature Dependence as Determined by Current Mode (CM) Method}

\subsubsection{Method Experimental Methods}

To determine the temperature dependence of the absolute scintillation yield with the SSW method required making assumptions about the temperature dependence of the PD quantum efficiency. Also, the decay modes (other than the primary two, $\tau_{1}$ and $\tau_{2}$ ) and the temperature dependence of the rise time $\tau_{\text {rise }}(T)$ were neglected by the SSW method due to both the complexity of accounting for them and the SSW method only being sensitive to characteristic times on the same order as the amplifier shaping times. Thus, it was assumed that the contribution of the minor decay modes and the rise time to the total charge created by a scintillation pulse were negligible. To assess the validity of the SSW method assumptions and approximations, an independent method was needed to measure the temperature dependence of the $\mathrm{CsI}(\mathrm{Tl})$ scintillation yield. The current mode (CM) method used as the independent method is not affected by very long or very short decay times and utilized a thermally-isolated photodetector. Consequently, the current mode method results do not suffer from the shortcomings of the SSW method.

The current mode method was carried out by placing a $0.7 \mathrm{mCi}{ }^{68} \mathrm{Ge}$ source near the $\mathrm{CsI}(\mathrm{Tl})$ crystal in the same thermally-insulated, temperature-controlled enclosure as was used for the emission spectra measurements. The monochromator was replaced by a cooled Hamamatsu R-2059 PMT at the focal point of the second quartz lens, as seen in Fig. 4.11. The PMT output current was measured with a Keithlcy 617 electrometer at a rate of about three times per second, averaged every ten seconds, and recorded in computer file along with the crystal temperature, as seen in Fig. 4.12. It can be assumed that this average PMT output current is directly proportional to the scintillation yield. The crystal temperature was controlled by a slightly different method than was used to make the emission spectrum measurements because thermoluminescence was observed to affect these measurements both while increasing temperature and at thermal equilibrium. These 


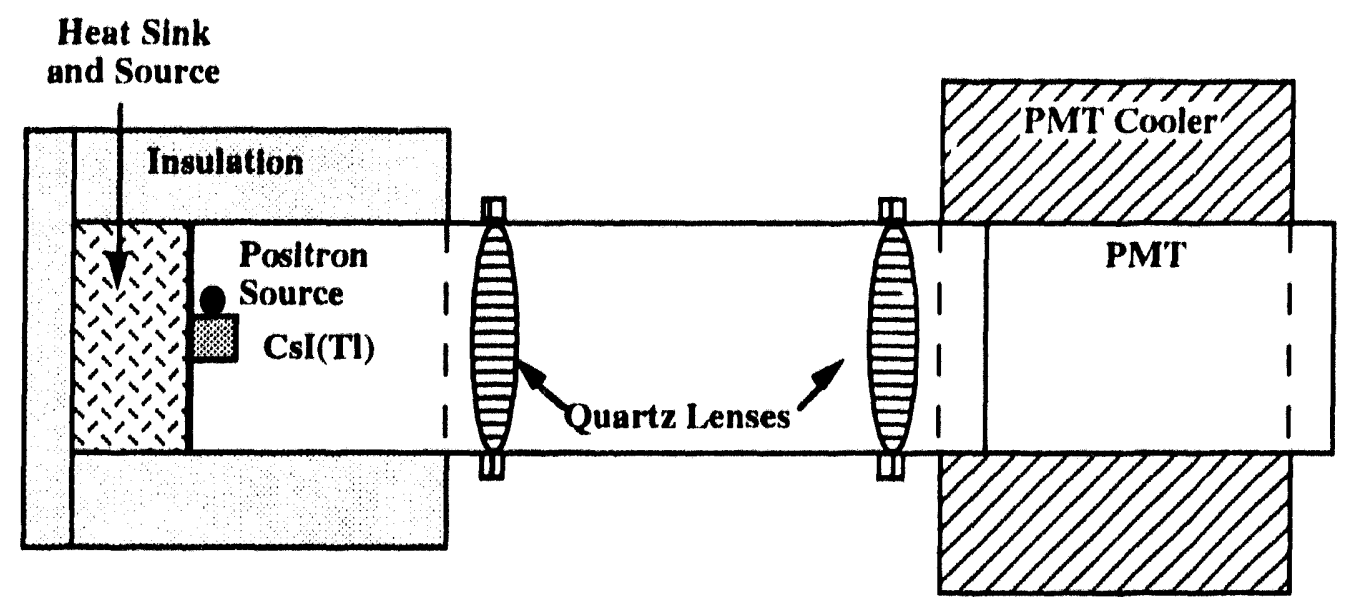

Figure 4.11: Detection system schematic for current mode method measurements.

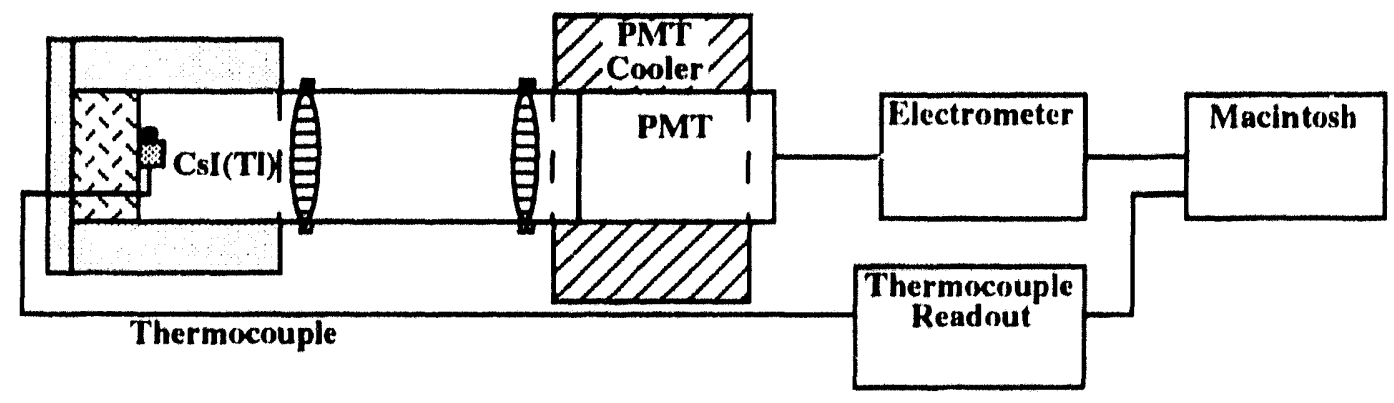

Figure 4.12: Detection system and electronics schematic for current mode method measurements.

thermoluminescence observations are further discussed in Chapter VI. To avoid these problems the crystal was initially heated to $50{ }^{\circ} \mathrm{C}$, the heating element turned off, and the crystal cooled at a rate of about $1^{\circ} \mathrm{C}$ per minute by manually regulating the liquid nitrogen flow such that the crystal temperature was monotonically decreased (temperature regulation method $C$, as seen in Fig. 4.13). The light collection efficiency and photodetector quantum efficiency were assumed to be independent of $\mathrm{CsI}(\mathrm{Tl})$ crystal temperature for the $\mathrm{CM}$ method. We believe that the temperature independence of the quantum efficiency is a very good assumption for the CM method because the PMT was thermally isolated from the crystal and thus maintained at a constant temperature. Additionally, the results of the CsI(Tl) emission spectrum temperature dependence in Chapter III suggest that changes in the wavelength-averaged quantum efficiency with temperature will be insignificant. 


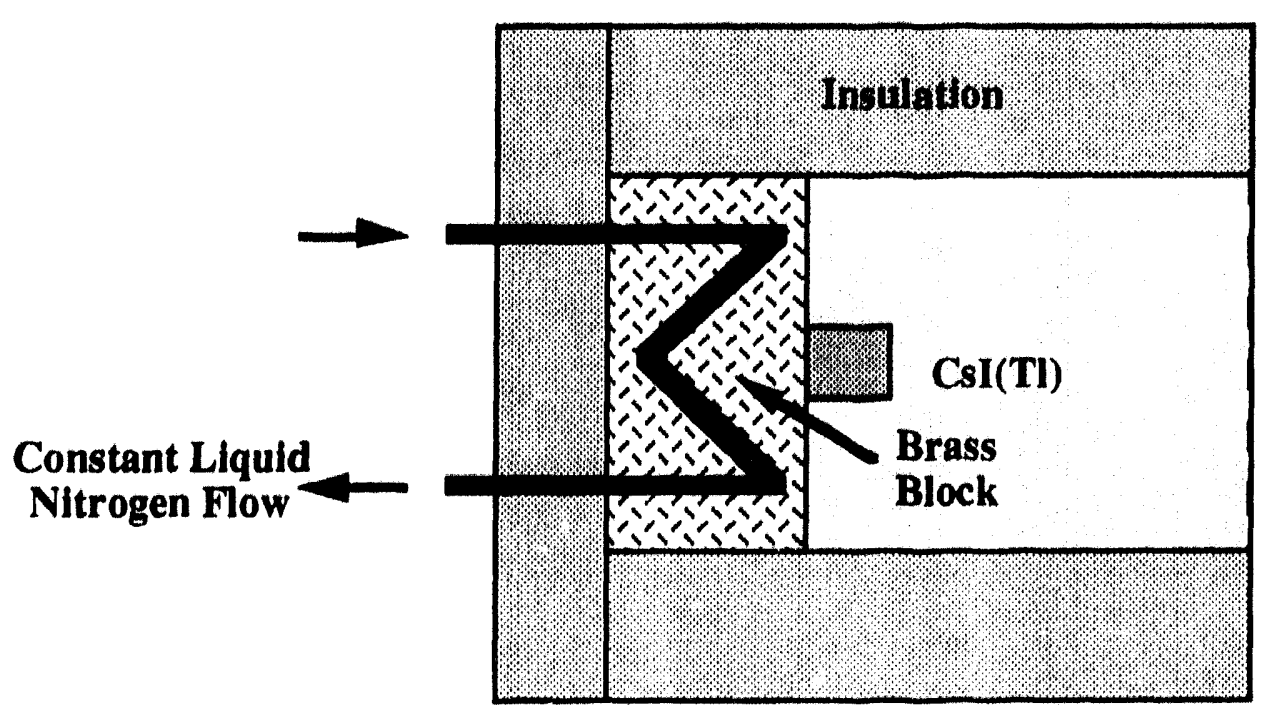

Figure 4.13: Temperature regulation method $C$.

\subsubsection{Method Results}

The PMT output currents are shown in Table 4.3 in $10^{\circ} \mathrm{C}$ increments for the CsI(Tl) crystal procured from STI, while the CM method results for all four crystals are shown in Fig. 4.14. The data in the figure are all normalized to unity at room temperature and thus represent relative scintillation yields. The scintillation yield is observed to peak between -35 and $-25^{\circ} \mathrm{C}$ for all four crystals between $5 \%$ and $8 \%$ above the room temperature yield. $\mathrm{At}-100^{\circ} \mathrm{C}$, the yield is seen to have decreased to about $64 \%$ of the room temperature yield for all crystals, while at $+50^{\circ} \mathrm{C}$ the yield is between $90 \%$ and $95 \%$ of the room temperature yield. In the range of -30 to $+50^{\circ} \mathrm{C}$, the scintillation yield is observed to be only slightly temperature dependent, varying from about $5 \%$ above to $10 \%$ below the room temperature yield, respectively. From the figure, we conclude that there is no significant difference in the temperature dependence of the scintillation yield from crystal to crystal.

\subsection{Absolute Scintillation Yield Temperature Dependence, $\mathrm{N}_{\mathrm{Abs}}(\mathrm{T})$}

Using the SSW method, light collection efficiency, and wavelength-averaged 
Table 4.3: Photomultiplier tube output current as a function of temperature for Solon Technologies, Inc. CsI(Tl) crystal.

\begin{tabular}{|c|c|}
\hline $\begin{array}{c}\text { Temperature } \\
\left({ }^{\circ} \mathrm{C}\right)\end{array}$ & $\begin{array}{c}\text { PMT Output Current } \\
(\mathrm{nA})\end{array}$ \\
\hline+50 & 4.09 \\
\hline+40 & 4.19 \\
\hline+30 & 4.26 \\
\hline+20 & 4.34 \\
\hline+10 & 4.40 \\
\hline 0 & 4.46 \\
\hline-10 & 4.51 \\
\hline-20 & 4.56 \\
\hline-30 & 4.59 \\
\hline-40 & 4.58 \\
\hline-50 & 4.38 \\
\hline-60 & 4.08 \\
\hline-70 & 3.71 \\
\hline-80 & 3.41 \\
\hline-90 & 3.03 \\
\hline-100 & 2.78 \\
\hline
\end{tabular}

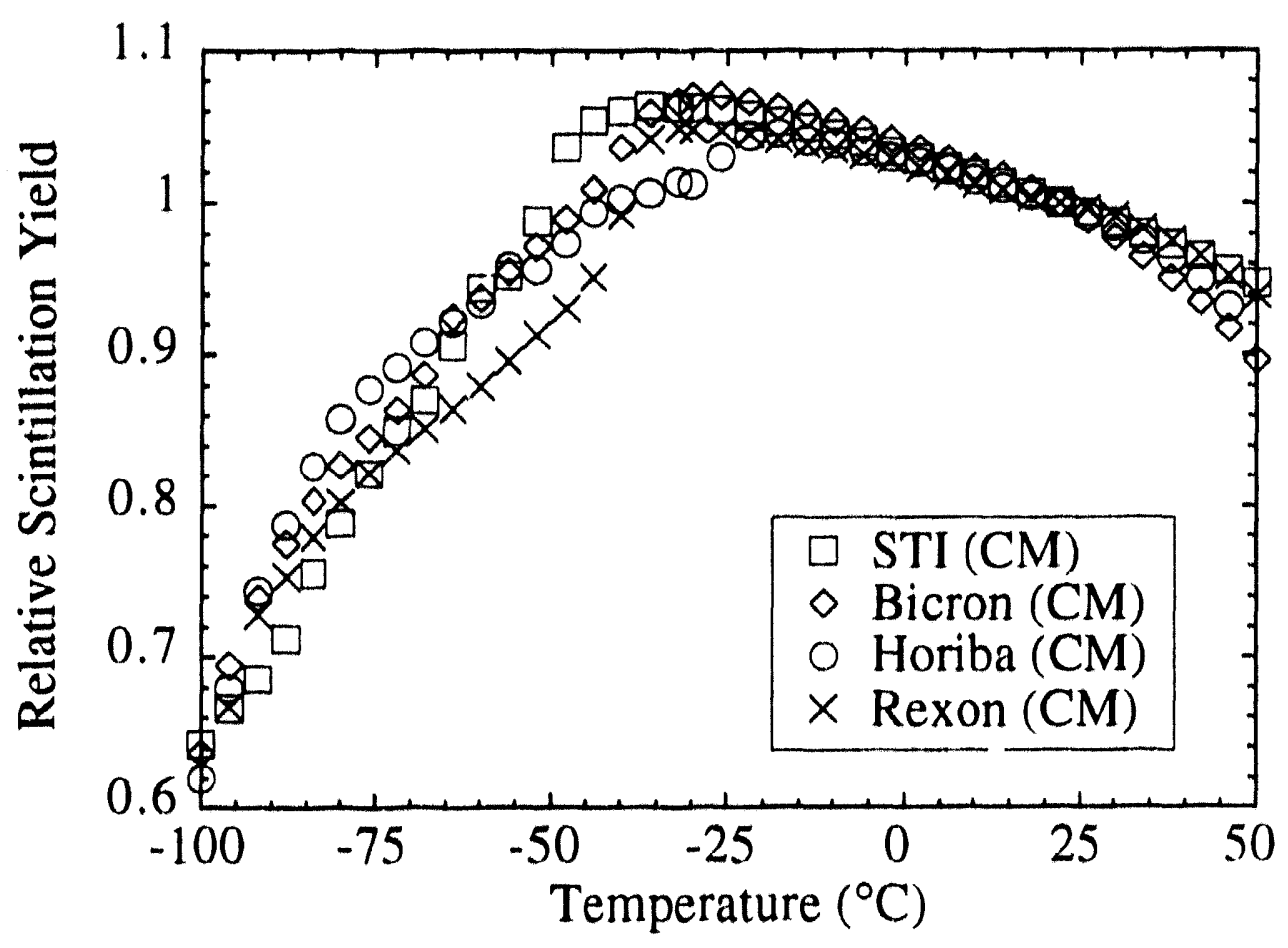

Figure 4.14: Temperature dependence of $\mathrm{CsI}(\mathrm{Tl})$ relative scintillation yield for crystals from Solon Technologies, Inc., Bicron Corp., Horiha Crystal Products, and Rexon Components, Inc., as measured by the current inode (CM) method. 
quantum efficiency results from Sections 4.1.3, 4.2, and 4.3 (Table 4.2, $\eta=0.814$, and $\bar{Q}=0.897)$, respectively, the temperature dependence of the CsI(Tl) absolute scintillation yield for gamma-rays is calculated using Eqn. (2.11) and is tabulated in Table 4.4. Additionally, the CsI(Tl) relative scintillation yield results from Section 4.4 .2 nomalized to the $20^{\circ} \mathrm{C}$ absolute scintillation yield determined by the SSW method are seen in Table 4.4 in $10^{\circ} \mathrm{C}$ increments, while Fig. 4.15 compares the results from the SSW and CM methods. The results from the two different methods agree to within $1 \%$ above $-10^{\circ} \mathrm{C}$, but are observed to diverge below this temperature. We believe that the divergence is due to not modeling the rise time of $\mathrm{CsI}(\mathrm{Tl})$ exactly at every temperature dependence with the SSW method, due to the degraded accuracy of the SSW method when $\tau_{2}$ becomes similar to the longest amplifier shaping time, and possibly due to not accounting correctly for the temperature dependence of the wavelength-averaged quantum efficiency in the SSW method. Section 5.2.3 contains a more detailed analysis of the problems associated with the SSW method at low temperatures. Since the CM method is unaffected by any rise and decay time variations and used a PMT that was held at a constant temperature, these problems do not affect the CM results. Furthermore, we believe that the CM method results are the most accurate representation of the $\operatorname{CsI}(\mathrm{Tl})$ scintillation yield temperature dependence. However, we also believe that the room temperature absolute scintillation yield $(65,500$ photons $/ \mathrm{MeV})$ determined by the SSW method is the most accurate value reported because we accounted for the ballistic deficit caused by $\tau_{2}$. We believe that $\eta, \bar{Q}$, and $\mathrm{N}_{\mathrm{cc}}$ have been accurately determined at room temperature and that it is only under the assumptions about temperature dependence that the SSW method has problems. It is observed that the scintillation yield peaks around $-35^{\circ} \mathrm{C}$ at about 69,300 photons $/ \mathrm{MeV}$, about 61,800 photons $/ \mathrm{MeV}$ at $+50^{\circ} \mathrm{C}$, and about 41,900 photons $/ \mathrm{MeV}$ at $-100^{\circ} \mathrm{C}$. Also, we see that the yield does not significantly change over the range of -40 to $+50^{\circ} \mathrm{C}$, varying from about $7 \%$ above to $6 \%$ below the room temperature scintillation yield.

The uncertainty in determining $\mathrm{N}_{\mathrm{Abs}}$ by the SSW method is the square root of the 
Table 4.4: CsI(Tl) absolute scintillation yield temperature dependence as determined by the shaped square wave (SSW) method and the current mode (CM) method for the Solon Technologies, Inc. crystal. The CM method data are normalized to $20^{\circ} \mathrm{C}$ yield determined by the SSW method.

\begin{tabular}{|c|c|c|}
\hline \multirow{2}{*}{$\begin{array}{c}\text { Temperature } \\
\left({ }^{\circ} \mathrm{C}\right)\end{array}$} & \multicolumn{2}{|c|}{ CsI(TI) Absolute Scintillation Yield } \\
\cline { 2 - 3 } & SSWMethod & CMMethod \\
\hline+30 & - & 61,800 \\
\hline+40 & - & 63,200 \\
\hline+30 & 64,400 & 64,400 \\
\hline+20 & 65,500 & 65,500 \\
\hline+10 & 67,300 & 66,400 \\
\hline 0 & 68,100 & 67,300 \\
\hline-10 & 68,100 & 68,100 \\
\hline-20 & 67,500 & 68,800 \\
\hline-30 & 66,600 & 69,300 \\
\hline-40 & 62,500 & 69,100 \\
\hline-50 & 56,400 & 66,100 \\
\hline-60 & 54,600 & 61,600 \\
\hline-70 & - & 55,900 \\
\hline-80 & - & 51,400 \\
\hline-90 & - & 45,700 \\
\hline-100 & - & 41,900 \\
\hline
\end{tabular}
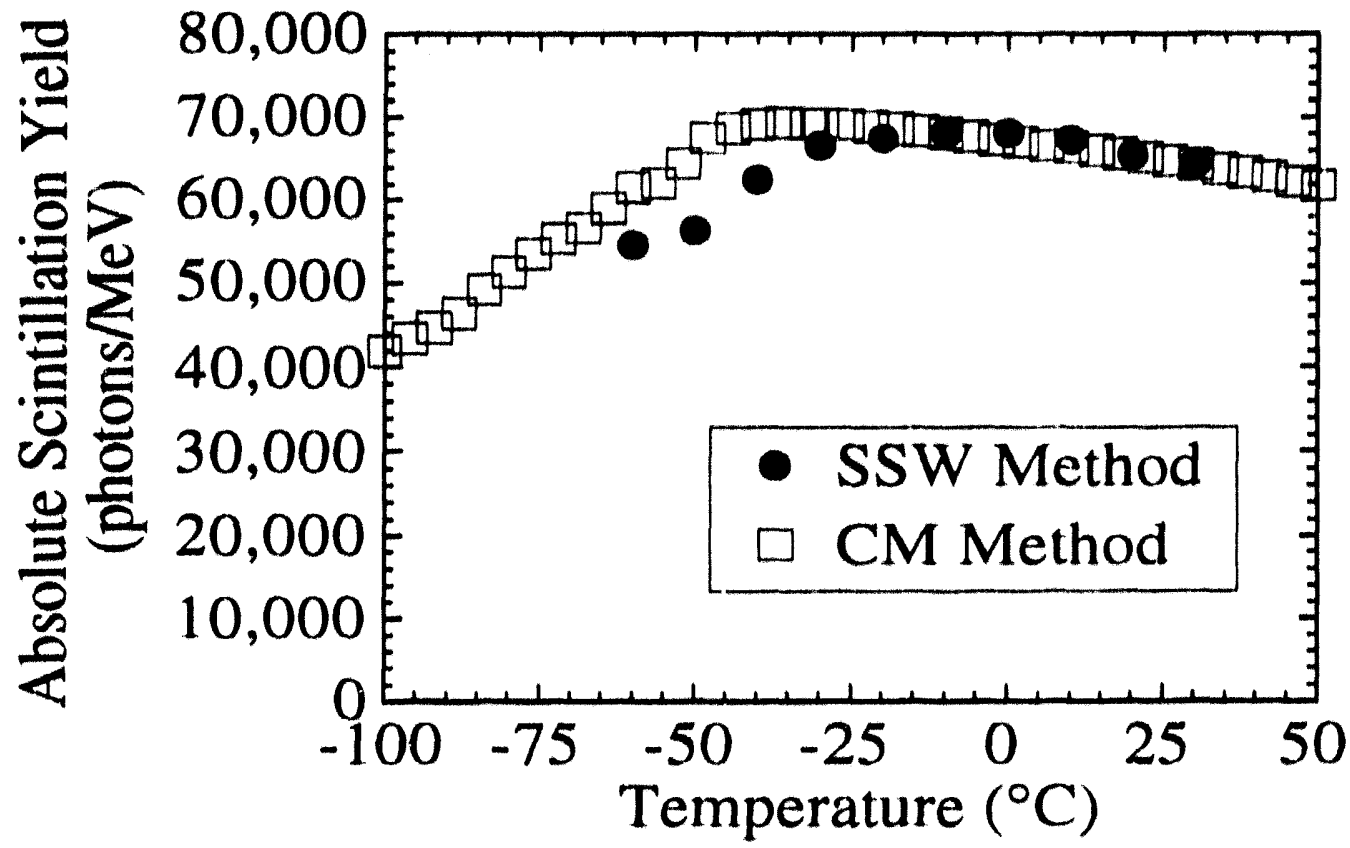

Figure 4.15: $\mathrm{CsI}(\mathrm{Tl})$ absolute scintillation yield temperature dependence for Solon Technologies, Inc. crystal, as determined by the shaped square wave (SSW) method and the current mode (CM) method. 
quadrature sum of the uncertainties associated with $\eta, \bar{Q}$, and $N_{c c}$ and is estimated to be $\pm 5 \%$. Since each of the uncertainties have been assumed to be independent of temperature, $5 \%$ is the estimated uncertainty of each value of $\mathrm{N}_{\text {Abs }}(\mathrm{T})$. This uncertainty coupled with the observed deviation in pulse height among the four different crystals of $3.6 \%$ (Fig. 4.7) suggests that we can expect about a $\pm 6.2 \%$ variation in the measured $\mathrm{N}_{\mathrm{Abs}}$ from crystal to crystal.

Although the CM method is not affected by changes in rise and decay times and the PMT is held at a constant temperature, the 400 S photocathode used with the R-2059 tube is significantly more sensitive to the $400 \mathrm{~nm}$ emission band of CsI(TI) than the $560 \mathrm{~nm}$ band. We estimate that the external quantum efficiency of the PMT is ahout $30 \%$ at $400 \mathrm{~nm}$ and about $5 \%$ at $560 \mathrm{~nm}$ from the specification sheets in the Hamamatsu catalog. Thus, the CM method results will be about six times more sensitive to changes in the amount of light emitted by CsI(T1) in the $400 \mathrm{~nm}$ band than in the $560 \mathrm{~nm}$ band. Recalling that the intensity from the $400 \mathrm{~nm}$ band varies significantly over the range of -100$)$ to +50$)^{\circ} \mathrm{C}$ (Figure 3.8 ) and effectively disappears between -50 and $-75^{\circ} \mathrm{C}$, the possibility arises that the variations observed in the scintillation yield using the CM method could be more representative of changes in the $400 \mathrm{~nm}$ emission band than the properly weighted emission spectrum of CsI(TI). Consequently, we have made a first order correction of the STI crystal CM method results for the spectral response of the PMT as shown in Fig. 4.16 to estimate this effect. Although the response of the R-2059 and the monochromator used for the emission spectrum measurements were calibrated, there is no simple means of decoupling the PMT and monochromator responses. Thus, the correction shown in Fig. 4.16 is based on the Hamamatsu specifications of the PMT response, not a calibrated response of the actual PMT used. From the figure, we see that with decreasing temperature the uncorrected $\mathrm{CM}$ method results are increasingly inaccurate, but that the scintillation yield still peaks at about the same temperature. The uncertainties shown in the Fig. 4.16 are based on an estimated uncertainty of about $0.5 \%$ for the integration of the PMT quantum efficiency over the 


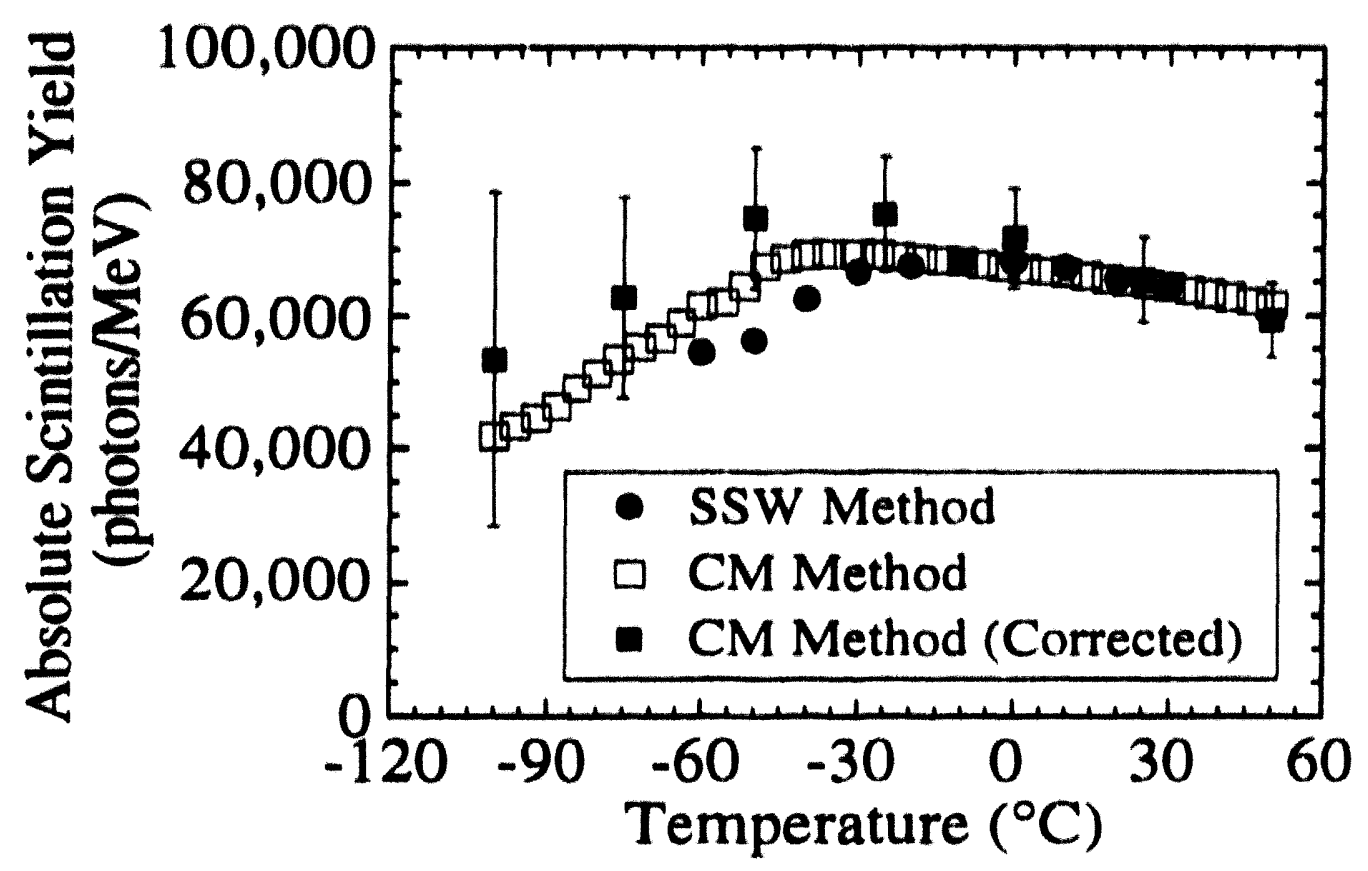

Figure 4.16: Comparison of shaped square wave (SSW) method and current mode (CM) method absolute scintillation yield results with $\mathrm{CM}$ method results corrected for response of Hamamatsu R-2059 (CM Corrected).

CsI(Tl) emission spectrum propagated through the calculations made to determine the correction factors. A photocathode with a flat response over the emission spectrum of CsI(TI) would yield the most accurate uncorrected CM method results, while a PMT with a calibrated response would allow proper correction of $\mathrm{CM}$ methnd data. We believe that the true scintillation yield of CsI(TI) lies within the uncertainties of the corrected data shown in Fig. 4.16, but that the uncorrected data represents the overall trends of the CsI(TI) scintillation yield accurately (i.e., temperature of maximum yi is and monotonic decrease in yield above and below the maximum).

One possible explanation for difference between the SSW and CM method results is that a significant fraction of the CsI(Tl) scintillation yield is contrihuted by decay modes with characteristic times much longer than $\tau_{2}$. The CM method is sensitive to all light emitted by the scintillator, while the SSW method was developed to only be sensitive to light emitted by the two primary decay modes. Light emitted with characteristic times on 
the order of milliseconds or longer are termed "afterglow" and observed to contribute about $9 \%$ of the total $\mathrm{NaI}(\mathrm{Tl})$ scintillation yield and typically about 0.1 to $0.8 \%$ of the CsI(TI) total yield [FAR82]. These long characteristic times are typically caused by impurities in the scintillator that create electron traps in the band structure. Thus "afterglow" decreases as crystal growing techniques improve and crystals are grown with fewer impurities. The luminescence that is described as "afterglow" is released when the electrons that are trapped at impurity sites are freed by thermal excitation. Thus, the characteristic times of "afterglow" decay components will increase exponentially with inverse temperature, as described in the Chapter V (more simply, the characteristic times increase with decreasing temperature). The impurity site electron traps are typically "deep" and thus the excitation energy necessary to free the electron is relatively large. Consequently, the characteristic times of "afterglow" components will increase orders of magnitude for relatively small decreases in temperature and become on the order of hours or days at low temperatures. When characteristic times hecome this long, the luminescence emitted as the result of impurity site electron traps is typically thermoluminescence (caused hy an increase in crystal temperature, further discussed in Chapter VI) instead of "afterglow". Since significant $\mathrm{CsI}(\mathrm{Tl})$ thermoluminescence has been observed helow $-20^{\circ} \mathrm{C}$, it is reasonable to assume that the room temperature "afterglow" components are the source. Furthermore, it is reasonable to assume that CsI(TI) "afterglow" does not significantly increase in the range of -100 to $+50^{\circ} \mathrm{C}$ and will not contribute more than about $1 \%$ of the total scintillation yield. Therefore the room temperature absolute scintillation yield determined hy the SSW method probably underestimates the yield by $\leq 1 \%$ and the divergence of the SSW and CM method results with decreasing temperature is probahly due to the problems with SSW method enumerated above.

On several occasions it has been mistakenly assumed that the reason that we have reported a larger scintillation yield for $\mathrm{CsI}(\mathrm{Tl})$ than previously reported is hecause we use a light collection efficiency of $81 \%$, where other authors have assumed near perfect light 
collection efficiency $(\eta=1,0)$. However, the same authors use a wavelength-averaged quantum efficiency of about 0.71 , such the product $\eta \bar{Q}$, which appears in the denominator of Eqn. (2.11), is about 0.71. Due to the definitions of $\eta$ and $\bar{Q}$ that we have adopted, our values of $\eta$ and $\bar{Q}$ are significantly different $(0.814$ and 0.897 , respectively), but the product is about 0.73 . Clearly, the difference between the resulting $N_{A b s}$ that we have reported and what others have reported is not significantly affected by our definitions or $\eta$ and $\bar{Q}$, and thus our scintillation yield is larger hecause we have accounted for the hallistic deficit.

Our room temperature absolute scintillation yield can be confirmed using the results presented by Bird, et al. [BIR92], since a very similar CsI(TI)/PD letector was used and they have conveniently quantified most of the differences. They report a value for $\mathrm{N}_{\mathrm{cc}}\left(\mathrm{E}_{\gamma} \mathrm{T}\right) / \mathrm{E}_{\gamma}$ at room temperature of $41,000 \mathrm{e}-\mathrm{h}$ pairs $/ \mathrm{MeV}$, arrived at using the direct interactions method of charge calibration and a $3 \mu \mathrm{s}$ amplifier shaping time. Additionally, the crystal was polished on all sides except the side opposite the PD which was roughened and all sides were wrapped with a white filter paper. Bird, et al. report that roughening the one side yields the optimum light collection efficiency and that a crystal with all six sides polished, like ours, was measured to yield about $95 \%$ of the optimum. They also report that wrapping the crystal with the filter paper yielded about $9 \%$ more light than when the crystal was wrapped with white PTFE tape like ours was. Using these two correction lactors we can estimate an effective light collection efficiency $\eta_{\text {eff }}$ based on the $\eta=(0,314$ as seen in Table 4.5. Referting to Table 4.1, we see that using the DI method at $3 \mu \mathrm{s}$ with our crystal resulted in about $36,700 \mathrm{e}-\mathrm{h}$ pairs/MeV. Thas, the absolute scintillation yield (not corrected for ballistic deficit) can be calculated for the $3 \mu \mathrm{s}$ DI method results from both Bird, et al. and this study. A correction factor for the ballistic deficit (1.302) can then be determined by dividing the true absolute scintillation yield (65,500 photons $/ \mathrm{MeV})$, determined using the SSW method, hy the yield determined for our DI method at $3 \mu \mathrm{s}$ $(50,300)$ photons $/ \mathrm{MeV})$. Table 4.5 tabulates the light collection efficiency and hallistic 
Table 4.5: Comparison of room temperature absolute scintillation yield determined in this study to results from Bird, et al. [BIR92] and Holl, et al. [HOL.88] by correcting for differences in light collection efficiency and ballistic deficit experienced in the direct interaction (DI) calibration method for an amplifier shaping time of $3 \mu s$.

\begin{tabular}{|c|c|c|c|c|}
\hline & $\begin{array}{c}\text { This Study } \\
\text { SSW }\end{array}$ & $\begin{array}{l}\text { This Study } \\
\text { DI@3 } 3 \text { s }\end{array}$ & $\begin{array}{l}\text { [BIR92] } \\
\text { DI@3 } 3 \mu \mathrm{s}\end{array}$ & $\begin{array}{l}\text { [HOL88] } \\
\text { DI@ } 3 \mu s\end{array}$ \\
\hline$N_{c e} / E_{\gamma}(e-h$ pairs/MeV) & $47,8(x)$ & 36,700 & $41.00(1)$ & - \\
\hline $\begin{array}{l}\text { Light Collection Efficiency, } \eta \\
\text { (This Study) }\end{array}$ & 0.814 & 0.814 & 0.814 & - -. \\
\hline $\begin{array}{l}\text { Correction for surface treatment, } \\
C_{8}\end{array}$ & - & - & 0.95 & - \\
\hline Correction for wrapping. $\mathrm{C}_{w}$ & 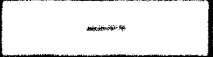 & - & 1.199 & - \\
\hline $\begin{array}{l}\text { Effective Light Collection } \\
\text { Efficiency, } \eta_{\text {orf }}=\eta\left(C_{w} / C_{n}\right)\end{array}$ & $-\infty$ & - & 11.934 & - \\
\hline Quantum Efficiency, $\bar{Q}$ & 0.897 & 01.897 & 0.897 & - \\
\hline 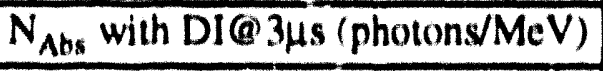 & $\cdots$ & $50,3(1)(1)$ & $48.9(10$ & $51,8(10$ \\
\hline $\begin{array}{l}\text { Ballistic Deficit Correction for } \\
\text { DI }(0) 3 \mu \mathrm{s}, C_{B D}=\frac{N_{\Delta b s}(S S W)}{N_{A b s}(D I \text { (Q } 3 \mu s)}\end{array}$ & - & 1.302 & 1.302 & $1.3(1) 2$ \\
\hline 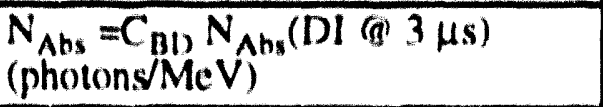 & $655(x)$ & $6.5 .5(1)(1)$ & $63.7(x)$ & $67.4(x)$ \\
\hline
\end{tabular}

deficit corrections. The corrected estimate of the absolute scintillation yield based on the results from Bird, et al. is $63,7(x)$ photons/MeV which is ahout $2.8 \%$ smaller than the $65.50(1) \pm 6.2 \%$ photons $/ \mathrm{MeV}$ that we are reporting. Consequently, the results of Bird, et al. [BIR92] seem to confirm our room temperature CsI(T1) ahsolute scintillation yicld.

To further confirm our results we can correct the previous Csl(Ti) absolute scintillation yicld that was most often quoted. Holl, et al. [H()L88| reported an absolute scintillation yield of $51,8(x)$ photons/MeV for Csl(Tl). They used methods similar to ours to determine the wavelength-averaged quantum efficiency and the light collection efficiency, but they used a $3 \mu$ s amplifier shaping time and the direct, iteraction method to calihrate pulse height to charge. When corrected for ballistic deficit using the same factor as used ahove, the absolute scintillation reported hy Holl, et al, is about 67,4(1) 


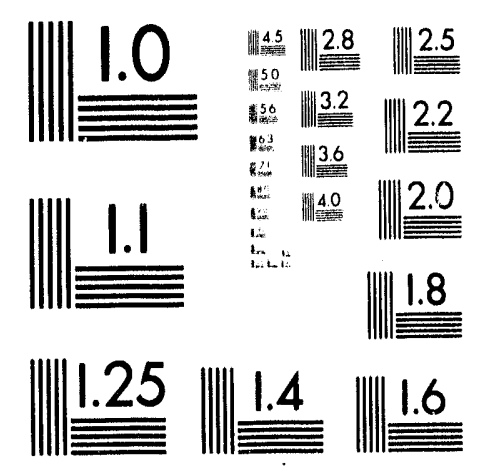



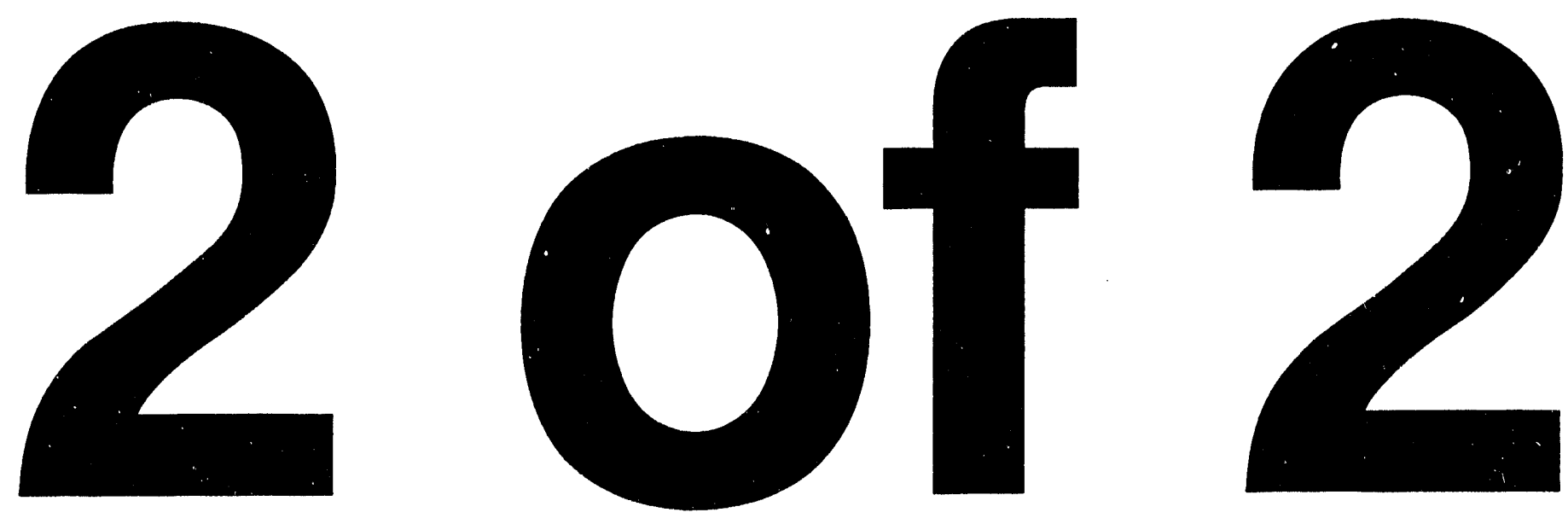
photons/MeV (Table 4.5). This result is about 3\% larger than our reported value, which is within both the $5 \%$ percent uncertainty and the expected crystal-to-crystal deviation of $3.6 \%$ that we have reported (thus, within the estimated total uncertainty for our value of $6.2 \%)$.

As mentioned in Chapter I, scintillation efficiency has been used as a figure-ofmerit for scintillators. By taking the product of the average scintillation photon energy and the absolute scintillation yield, the scintillation efficiency can be calculated. The average scintillation photon energy of $\mathrm{CsI}(\mathrm{Tl})$ was determined by calculating the first moment of the STI crystal emission spectrum with a result of $2.3 \mathrm{eV}$, or about $540 \mathrm{~nm}$. The resulting $\operatorname{CsI}(\mathrm{Tl})$ scintillation efficiency is 0.151 , or $15.1 \%$. For many years it was believed that $\mathrm{NaI}(\mathrm{Tl})$ was the most efficient scintillator with a reported value of 0.141 by Van Sciver and Bogart [VAN57] and values as high as 0.135 by Sakai [SAK87], but when the absolute scintillation yield of $\mathrm{CsI}(\mathrm{Tl})$ is accurately determined we see that $\mathrm{CsI}(\mathrm{TI})$ is about $7 \%$ more efficient at converting gamma-ray energy to scintillation photon energy.

Previously published CsI(TI) scintillation yield temperature dependence results have been based on measuring the photopeak centroid as a function of temperature with a single amplifier shaping time. Fig. 4.17 shows our measurement of the photopeak centroid variation with temperature for 2,5 , and $12 \mu$ s shaping times. The data are normalized to unity at room temperature. Fig. 4.18 compares previously published results with our $2 \mu \mathrm{s}$ data. Note that the $2 \mu$ s data reproduces the previously reported values. Note also that the temperature dependence of the photopeak centroid data in Fig. 4.17 varies significantly with shaping time. The photopeak centroids are observed to increase with increasing shaping time and the spread becomes larger at lower temperatures. This spread is due to neglecting the ballistic deficit and results in an inaccurate representation of the CsI(Tl) scintillation yield. Consequently, we believe that the results presented in Fig. 4.15 represent the most accurate values of the absolute scintillation yield temperature dependence of $\operatorname{CsI}(\mathrm{Tl})$. 


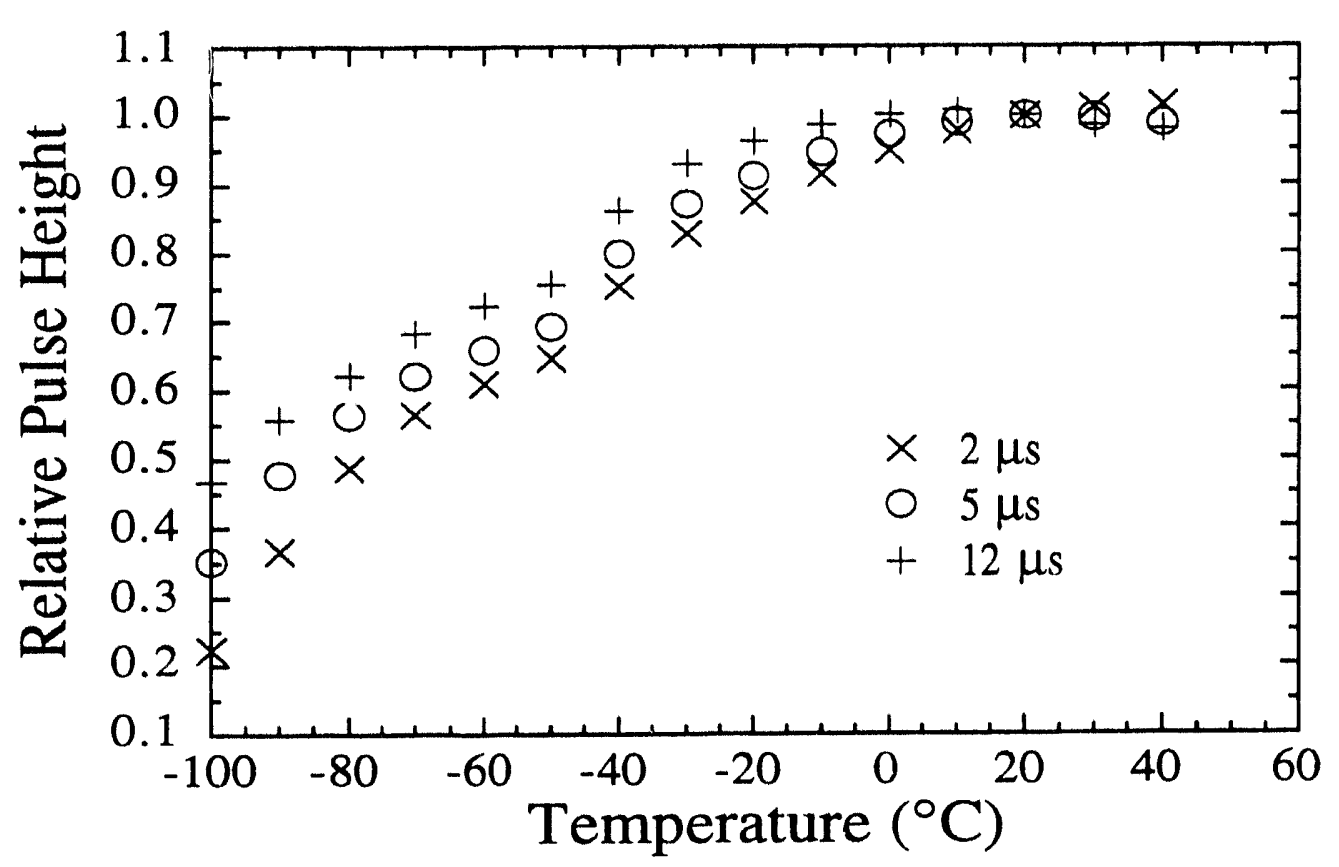

Figure 4.17: Temperature dependence of $\mathrm{CsI}(\mathrm{Tl})$ pulse height for amplifier shaping time constants of 2,5 , and $12 \mu \mathrm{s}$.

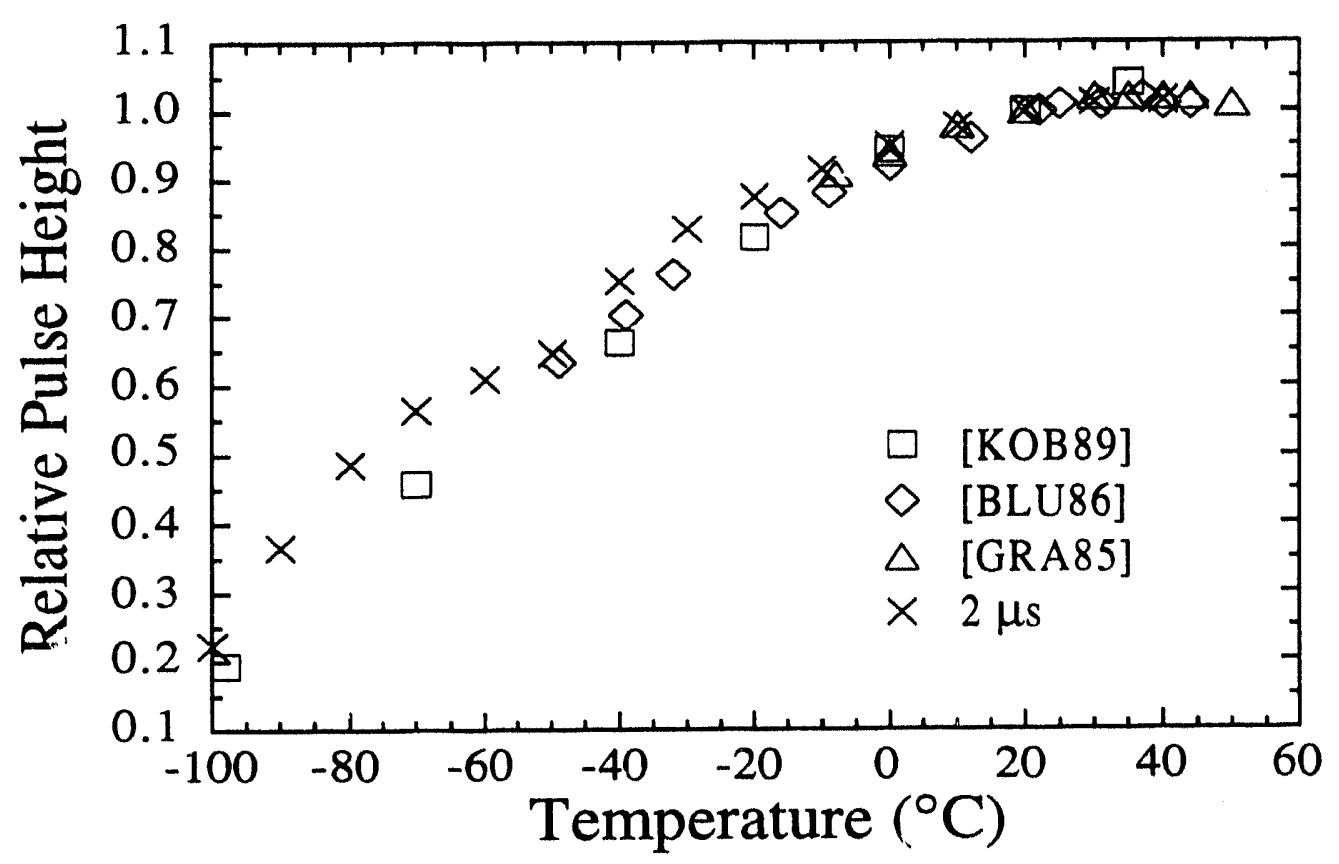

Figure 4.18: Previously reported CsI(Tl) scintillation yield temperature dependence, measured using a single amplifier shaping time, compared to $2 \mu$ s pulse height temperature dependence from this study. 
Our original interest in the temperature dependence of the CsI(T1)/PD detector was to see if the energy resolution would improve significantly with decreasing temperature due to the decrease in PD dark current. When looking at the temperature dependence of the scintillation yield and considering the improvement of the PD with decreasing temperature, the initial implication is that the energy resolution should be optimized around the temperature of maximum scintillation yield. However, the photopeak centroid decreases with tempurature below about $+10^{\circ} \mathrm{C}$, as seen in Fig. 4.17, even though the scintillation yield is observed to increase with decreasing temperature to about $-35^{\circ} \mathrm{C}$ (Fig. 4.15 ). The reason for the difference is that the ballistic deficit is not allowing the full integration of the CsI(Tl) scintillation yield. Consequently, we found that the energy resolution was optimized around $0^{\circ} \mathrm{C}$ with only a slight improvement over the room temperature energy resolution. Therefore, the temperature dependence of the pulse height, as reported in Figs. 4.17 and 4.18 , is actually the data of ultimate interest for practical applications using these shaping time constants since most applications use pulse height to acquire and analyze data. However, it is misleading and incorrect to report the pulse height data as the scintillation yield. While the pulse height data is the usable and more practical information, we believe that we have reported the fundamental characteristic of $\mathrm{CsI}(\mathrm{Tl})$ and that systems may be designed to utilize the absolute scintillation yield of $\mathrm{CsI}(\mathrm{Tl})$ in the future. Since the pulse height behavior will be dependent on the detector geometry and the data acquisition system (e.g., shaping time constant), the pulse height data should be measured for each system configuration. 


\section{CHAPTER V \\ RISE AND DECAY COMPONENTS}

The rise and decay time constants of a scintillator will largely determine the counting rate limitations of a detector that uses that specific material and the timing capabilities of the material if it were to be used as the source of a timing signal. The values and number of the decay times of CsI(Tl) have been controversial issues through the years (as mentioned in Chapter I) due to a relatively long decay time that has a relatively low initial intensity and thus can easily be mistaken for background. Additionally the $40 \mathrm{~ns}$ rise time at room temperature reported by Grassmann, et al. [GRA85] is much longer than has been observed for any other scintillating material [DER90, DER92], but has not been reported by others. Additionally, the rise and decay time constants, $\tau_{j}$, are expected to hehave like

$$
\tau_{j} \propto e^{E_{j} / k T}
$$

where $E_{j}$ is the activation energy of the $j^{\text {th }}$ process and $k$ is the Boltzmann constant [BIR64]; thus, it is expected that the characteristic times will increase with decreasing temperature. Furthermore, the rise time and decay times and their temperature dependence directly affect the operational characteristics of $\mathrm{CsI}(\mathrm{Tl})$ and had not been sufficiently determined prior to Valentine, et al. [VAL93a]; these results are presented in the remainder of this chapter.

The temporal luminescence intensity of $\operatorname{CsI}(\mathrm{Tl}), \mathrm{I}(\mathrm{t})$, (as defined by Eqn. (2.3))

was determined by the modified Bollinger-Thomas method [BOL61, MOS77] (as discussed in Section 2.3.1), while the primary contributions to $I(t)$ for $\operatorname{CsI}(T l)$ (as defined by Eqn. (2.12)) were determined by the shaped square wave (SSW) method [VAL92a] as 
discussed in Section 2.2.3. The modified Bollinger-Thomas (MBT) method has become the accepted technique for determining $I(t)$, and was thus used to determine the most accurate estimates of all rise and decay times and the relative contribution of each luminescence mode. Additionally, the MBT method was used to confirm the applicability of determining the parameters of the primary contributions to $I(t)$ for CsI(Tl), Eqn. (2.12), hy the SSW method.

\subsection{Experimental Methods}

\subsubsection{Modified Bollinger-Thomas Method}

As seen in Fig. 5.1, the modified Bollinger-Thomas method used a barium fluoride $\left(\mathrm{BaF}_{2}\right)$ scintillating crystal coupled to a Hamamatsu R-2059 PMT to provide a trigger, or start signal. Another R-2059 PMT coupled to the CsI(Tl) crystal provided the stop signal. The "poor" optical coupling between the CsI(Tl) crystal and the PMT required for the MBT method was provided by separating the crystal and PMT by about $50 \mathrm{~cm}$ and by placing a sheet of aluminum foil with a small aperture over the entrance to the PMT cooler assembly. The $\mathrm{BaF}_{2}$ and $\mathrm{CsI}(\mathrm{Tl})$ were aligned with a $511 \mathrm{keV}$ annihilation photon source between them to excite both crystals simultaneously. Timing signals were generated from the PMT outputs by a Tennelec TC-454 quad constant fraction discriminator. The time difference hetween the start and stop signals was then determined by a Tennelec TC-862 time-toamplitude converter (TAC) and digitized by a LeCroy 3512 analog-to-digital converter, as shown in Fig. 5.2. The impulse response function of this system, $G(\mathfrak{t})$ in Eqn. (2.22), was determined by measuring the response to an ultra-fast scintillator $(\mathrm{ZnO}(\mathrm{Ga})$, which has a 600 ps decay time), and subsequently fitting the data while holding the scintillator parameters constant and allowing the response function parameters to vary. The full-width at half maximum of the resulting response function (or timing resolution) was found to be about 500 ps. (The impulse response function is quantified in Appendix D.) Consequently, the lower limit for measuring rise and decay times hy the MBT method is 

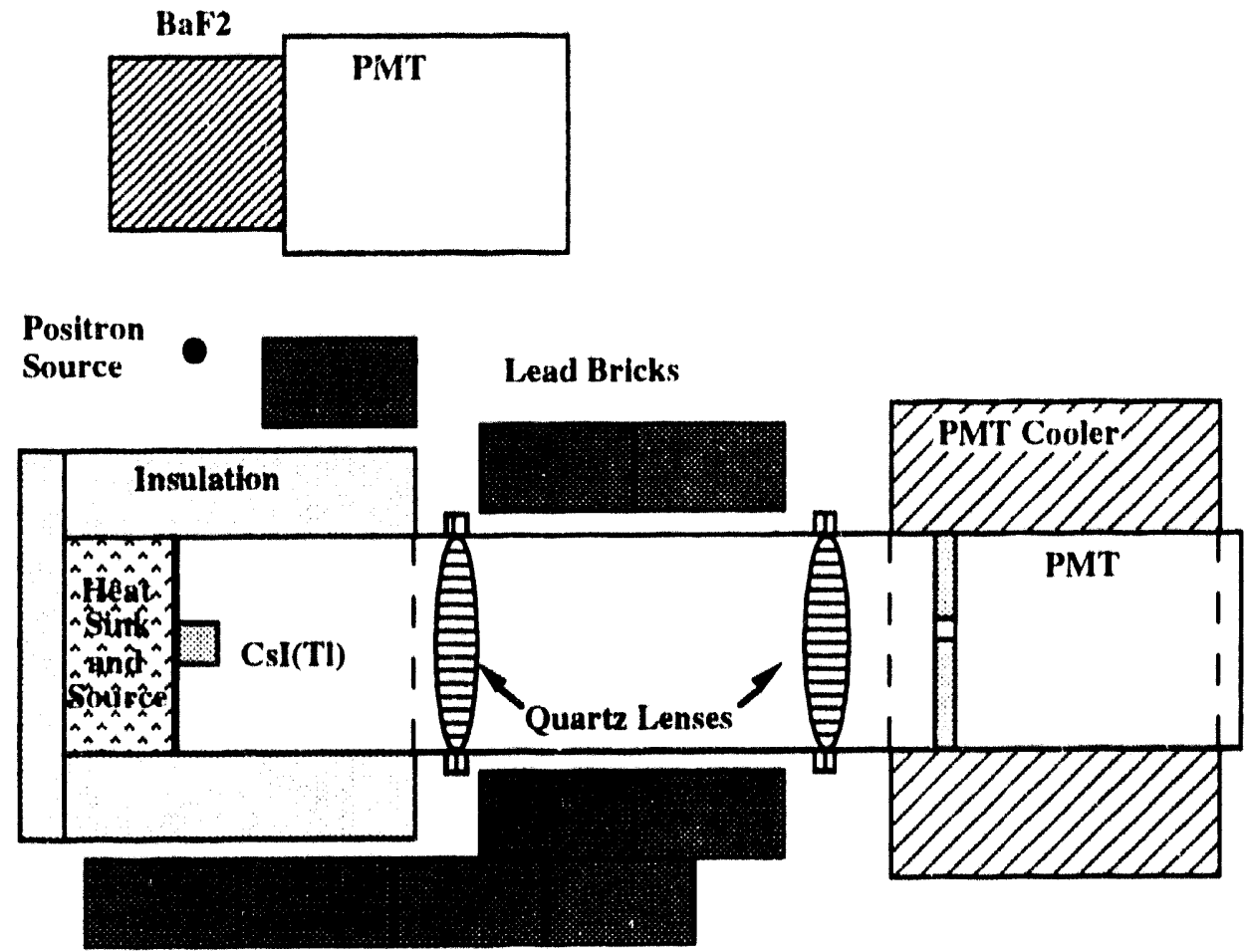

Figure 5.1: Detector geometry for modified Bollinger-Thomas method measurements.

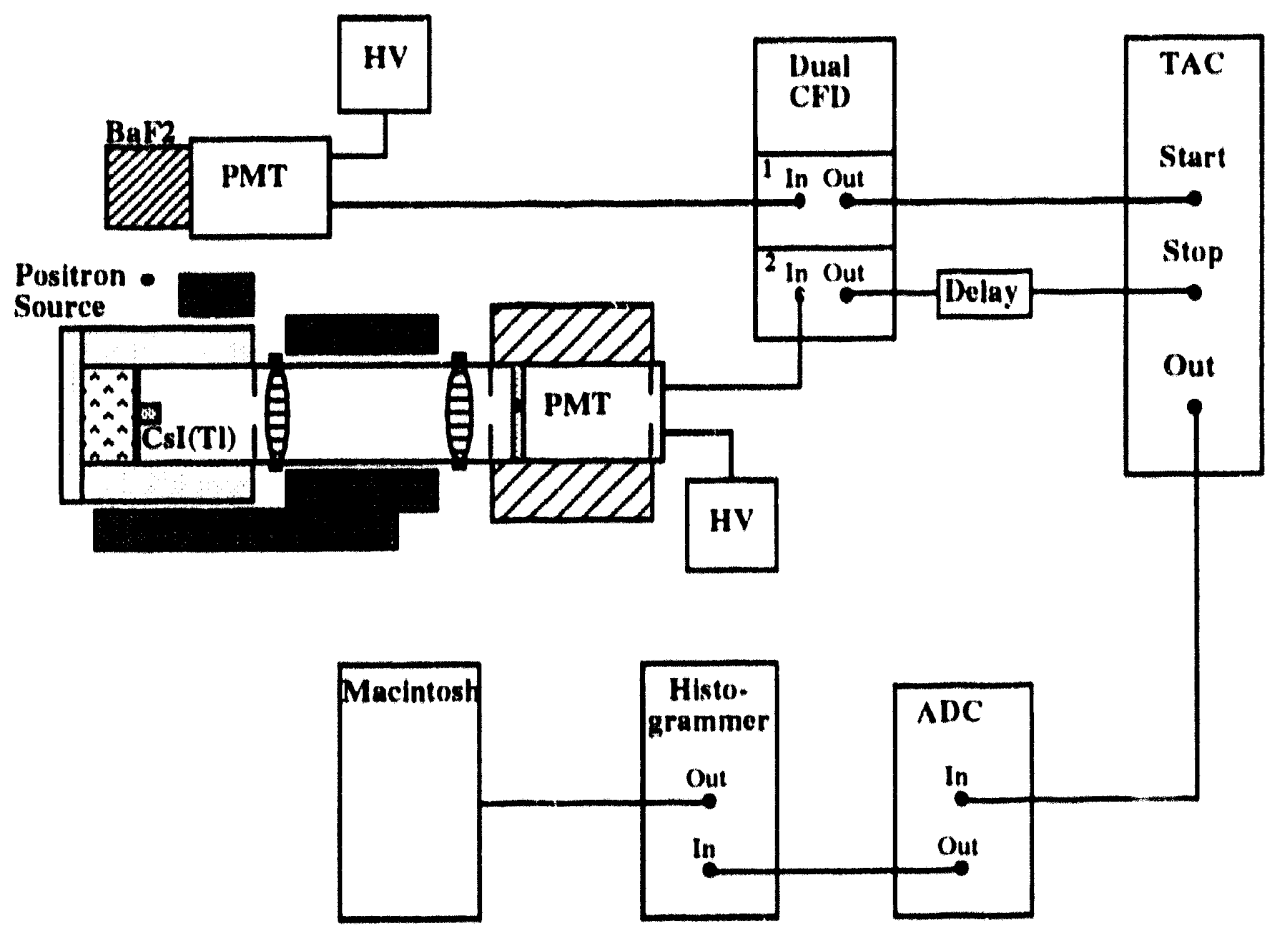

Figure 5.2: Electronics schematic for modified Bollinger-Thomas method measurements. 
50() ps. The crystals and first focusing lens were placed in the same thermally-insulated, temperature-controlled enclosure as was used for the emission spectrum measurements (described in Section 3.2), and temperature regulation method $A$ was used (Fig. 3.2).

To assess the crystal-to-crystal variations in the $\mathrm{CsI}(\mathrm{Tl})$ temporal luminescence yield, the MBT method was used with the four different crystals at room temperature and at ()$^{\circ} \mathrm{C}$. As noted in Section 3.1, the STI crystal was randomly chosen to determine the lemperature dependence of the temporal luminescence intensity over the temperature range of -100$)$ to $+5\left(0^{\circ} \mathrm{C}\right.$ in $10^{\circ} \mathrm{C}$ increments.

\subsubsection{Shaped Square Wave Method}

The experimental results used to determine the temperature dependence of the e-h pair yield (described in Section 4.1.3) were used to estimate $\tau_{1}, \tau_{2}$, and $q_{2} / q_{1}$ hy the SSW method. The potentiometers used for the integration stage RC time constants and the altenuation factor $k_{1}$ in the SSW circuit (Fig. 4.1 and Eqn. (2.21)) were calibrated with an oscilloscope. When the requirements of the SSW method were met (the photopeak and calibration pulse peak centroids occurring in the same channel for each available amplifier shaping time constant, see Fig. 4.6), the potentiometer settings were converted to decay lime constants and the charge ratio.

While the MBT measurements yield the rise time and any decay times of Csl('Tl) as a function of temperature, the SSW method can also be used as an independent method of determining the temperature dependence of the characteristic times. However, the SSW method is only sensitive to rise and decay times on the order of the amplifier shaping times (for the Canherra 2022 linear amplifier used, $250 \mathrm{~ns}$ to $12 \mu \mathrm{s}$ ). Therefore, the rise time and any decay time shorter than $\tau_{1}$ were not determined by this method. Similarly, the SSW method is not applicable when $\tau_{2}$ hecomes longer than amplifier shaping limes. Consequently, even though we tried to avoid using any a priori values when implementing the SSW method, it was necessary to use a rise time constant of ahout 20 ns to account for 
the exponential population of the $\tau_{1}$ luminescence mode at every temperature. The variations in the rise time with temperature were assumed to have a negligible affect on the total charge created by a CsI(T1) scintillation pulse. Any decay times shorter than $\tau_{1}$ were similarly assumed to contribute a negligible fraction of the charge to the scintillation pulse. Consequently, only $\tau_{1}, \tau_{2}$, and $\mathrm{q}_{2} / \mathrm{q}_{1}$ for $\operatorname{CsI}(\mathrm{Tl})$ can be determined by the SSW method with commercially-available amplifiers.

Photopeak centroids were acquired at ten different amplifier shaping time constants for all four crystals at room temperature to investigate the crystal-to-crystal variations in $\tau_{1}$, $\tau_{2}$, and $\mathrm{q}_{2} / \mathrm{q}_{1}$ observed during the MBT method measurements. The STI crystal was used to determine the temperature dependence of $\tau_{1}, \tau_{2}$, and $\mathrm{q}_{2} / \mathrm{q}_{1}$ over the range of -100 to $+50^{\circ} \mathrm{C}$ in $10^{\circ} \mathrm{C}$ increments by the SSW method.

\subsection{Results}

\subsubsection{Modiffed Bollinger-Thomas Results}

The most reliable fitting results for the modified Bollinger-Thomas method are achieved with a TAC range $>3 \tau_{j}$ and a bin width $<0.1 \tau_{j}$. Since decay time constants as short as $<0.5 \mathrm{~ns}$ and as long as $18 \mu \mathrm{s}$ were observed, these constraints would require taking data in $50 \mathrm{ps}$ bins over a $50 \mu \mathrm{s}$ range. The dynamic range of the TAC used was not large enough for this to be possible, thus data were acquired with two TAC ranges. Timing spectra were taken with a $1 \mu \mathrm{s}$ TAC range in $0.8558 \mathrm{~ns}$ bins at each temperature to accurately determine any exponential components with characteristic times of several hundred nanoseconds or less. For $\mathrm{T}>-20^{\circ} \mathrm{C}$, data were also taken with a $20 \mu \mathrm{s} \mathrm{TAC}$ range in $16.64 \mathrm{~ns}$ bins, and for $\mathrm{T} \leq-20^{\circ} \mathrm{C}$ with a $50 \mu \mathrm{s}$ range with $10.35 \mathrm{~ns}$ bins. These longer TAC ranges allowed the accurate determination of components with $1.20 \mu \mathrm{s}$ characteristic times. The system was calibrated for each TAC range separately by using a pulser and six 
different stop signal delays. A linear least squares fit of the six data points was used to determine the bin width for each TAC range.

The reported parameter values were determined through an itcrative process of fitting the long and short TAC range data. First, the longer TAC range data was fit by allowing all of the parameters to vary (an initial best estimate of the "longer" decay components). The resulting values were then used as starting values for the $1 \mu$ s TAC range data, but only the parameters associated with the "shorter" components, less than ahout one microsecond, were allowed to vary (an initial best estimate of the rise and shorter decay components). Subsequent estimates of the "longer" and "shorter" parameters were made using the previous hest estimates of the short and long range TAC data, respectively, IIntil hoth sets converged. In most cases only a few iterations were required.

Fig. 5.3 shows both the $1 \mu \mathrm{s}$ and $20 \mu \mathrm{s}$ TAC range timing spectra at room lemperature for the STI crystal. Along with decay times $\tau_{1}$ and $\tau_{2}, 772 \pm 8 \mathrm{~ns}(61.1 \%)$ and $3.53 \pm() .10 \mu \mathrm{s}(38.7 \%)$, respectively, an ultra-fast component and a $22.7 \pm 1.0 \mathrm{~ns}$ rise time that populates $\tau_{1}\left(I_{1}=-I_{\text {rise }}\right)$ are observed. The ultra-fast component appears as the three high channels at the heginning of the $1 \mu$ s TAC range spectrum and is not observed in the 20) $\mu$ s TAC range data due to its small fraction of the total luminescence yicld and the large hin width. The ultra-fast component has a decay time <().5 $\mathrm{ns}(0.5 \mathrm{~ns}$ is the timing resolution of the system used and thus the minimum decay time that can be accurately determined) and yields about $(0.2 \%$ of all of the photons (ahout $1(x)$ photons/MeV). Of the several hundred scintillators studied by Derenzo, et al. (DER9(), DER92], none were ohserved to have a rise time as long as we report here. Thus, we helieve that the rise time of $\mathrm{CsI}(\mathrm{Tl})$ at room temperature is longer than that of any other known scintillator. Tahle 5.1 compares the best fit $a_{j}$ 's and $\tau_{j}$ 's for the four different crystals at room temperature and $11^{\circ} \mathrm{C}$. For $\mathrm{T} \leq-30^{\circ} \mathrm{C}$, additional decay components $(j=4,5)$ were ohserved and are believed to be attributed to the luminescence of pure Csl. These decay components and the other fit results for all temperatures for the STI crystal are shown in Tahle 5.2. The lits 


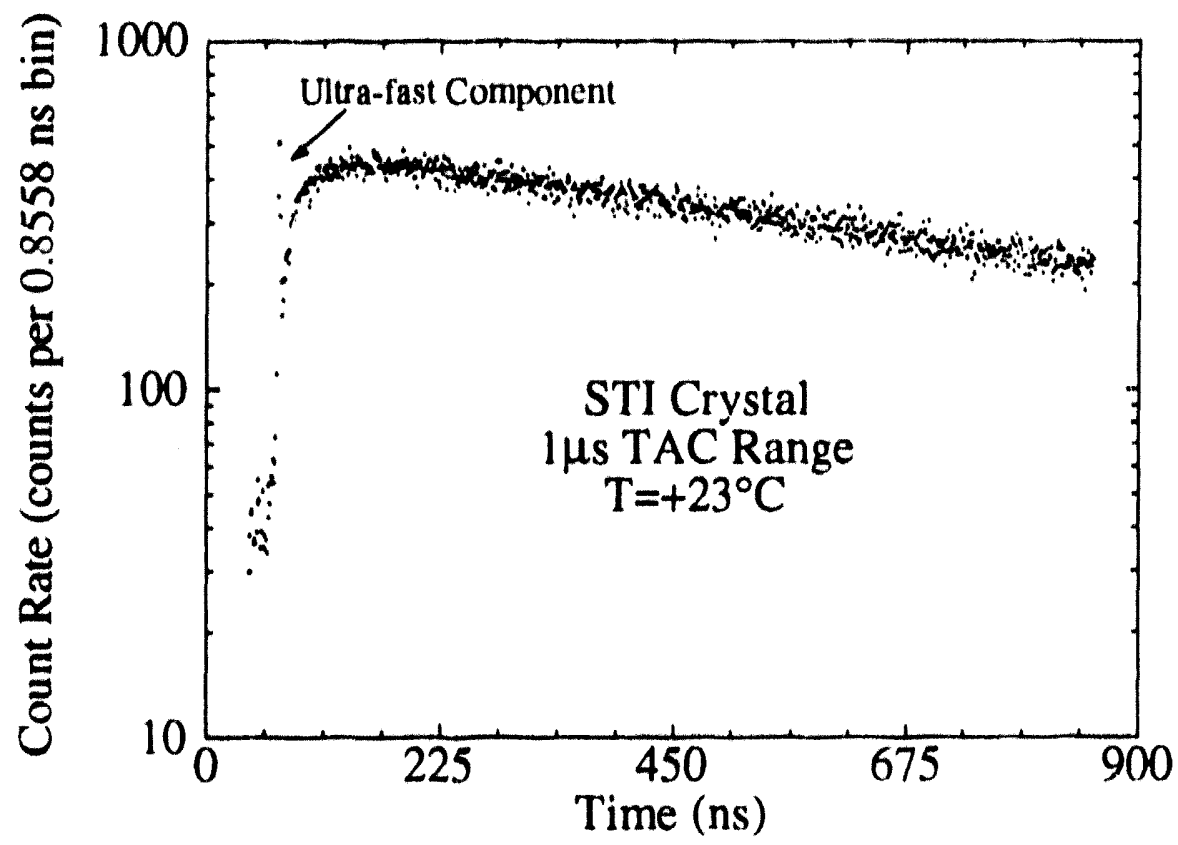

(a)

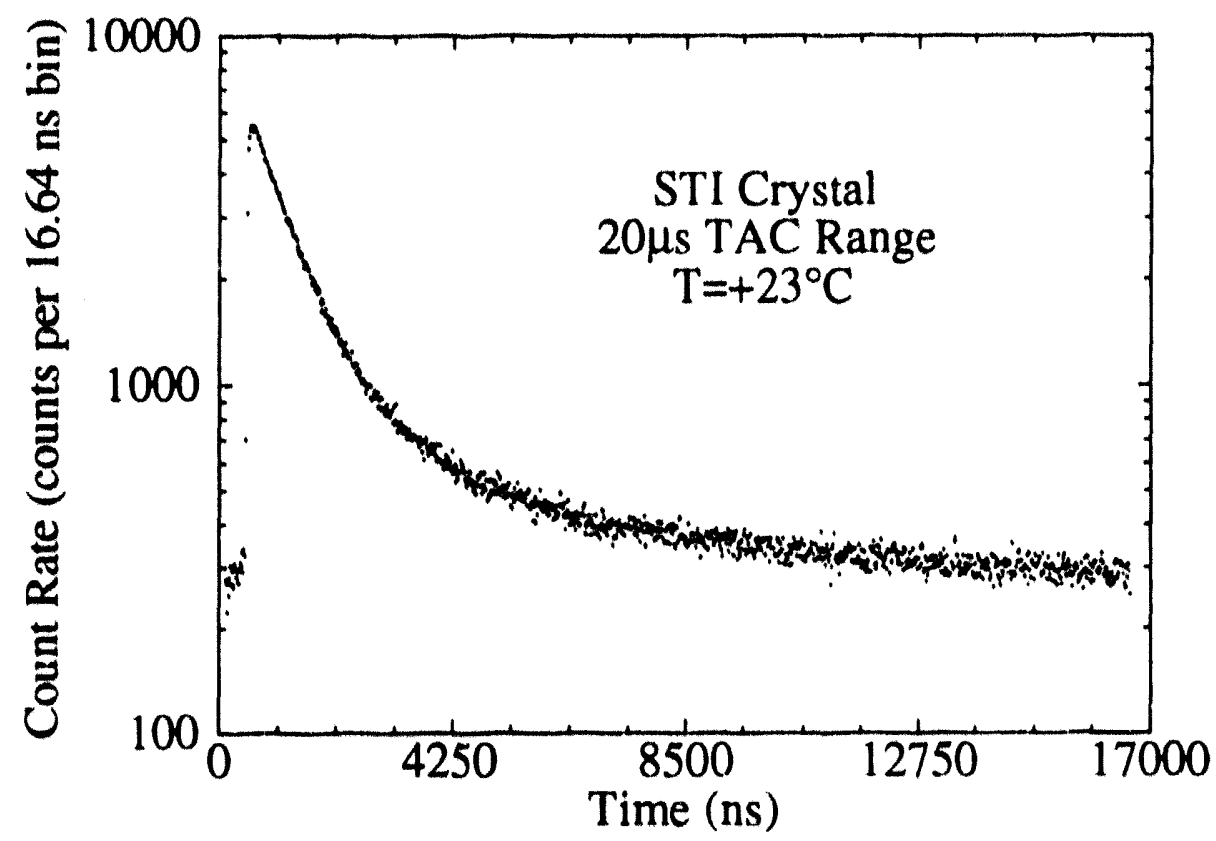

(b)

Figure 5.3: Gamma-ray excited CsI(Tl) luminescence timing spectra at room temperature for Solon Technologies, Inc. crystal for (a) short and (b) long TAC range data acquisition. 


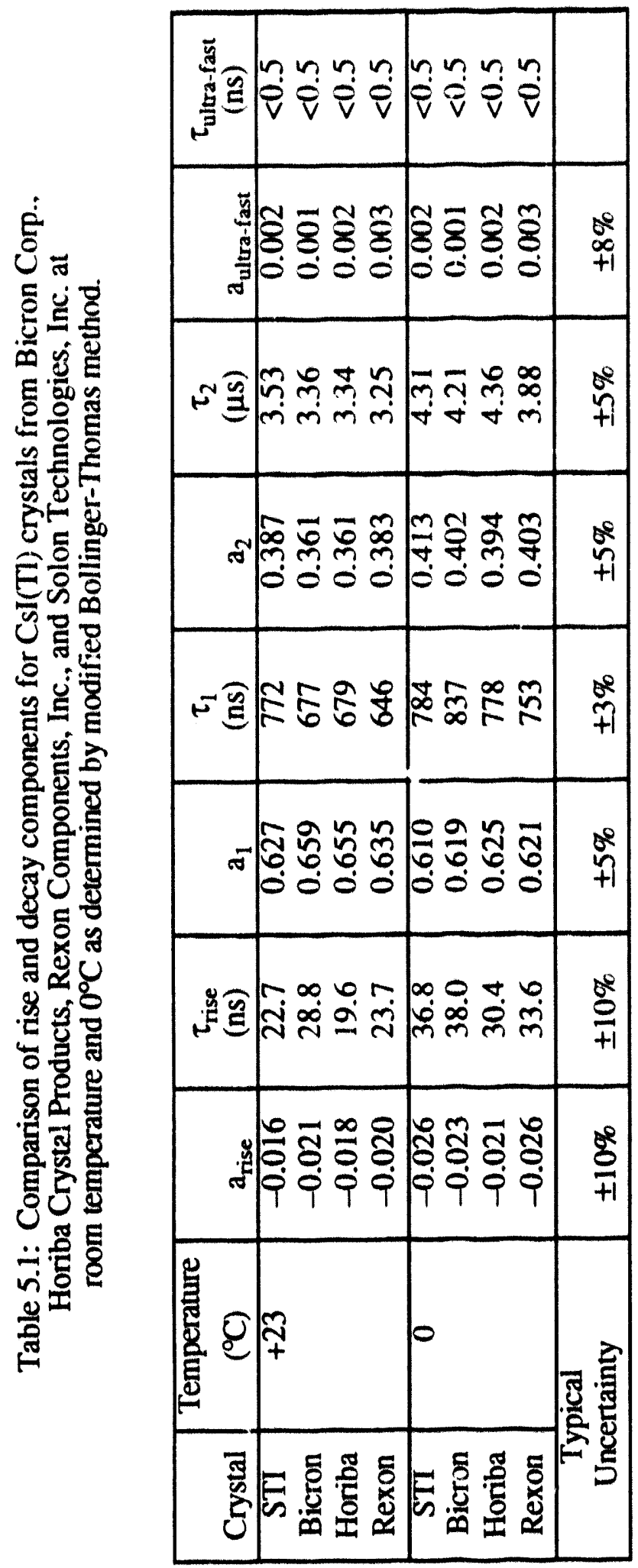




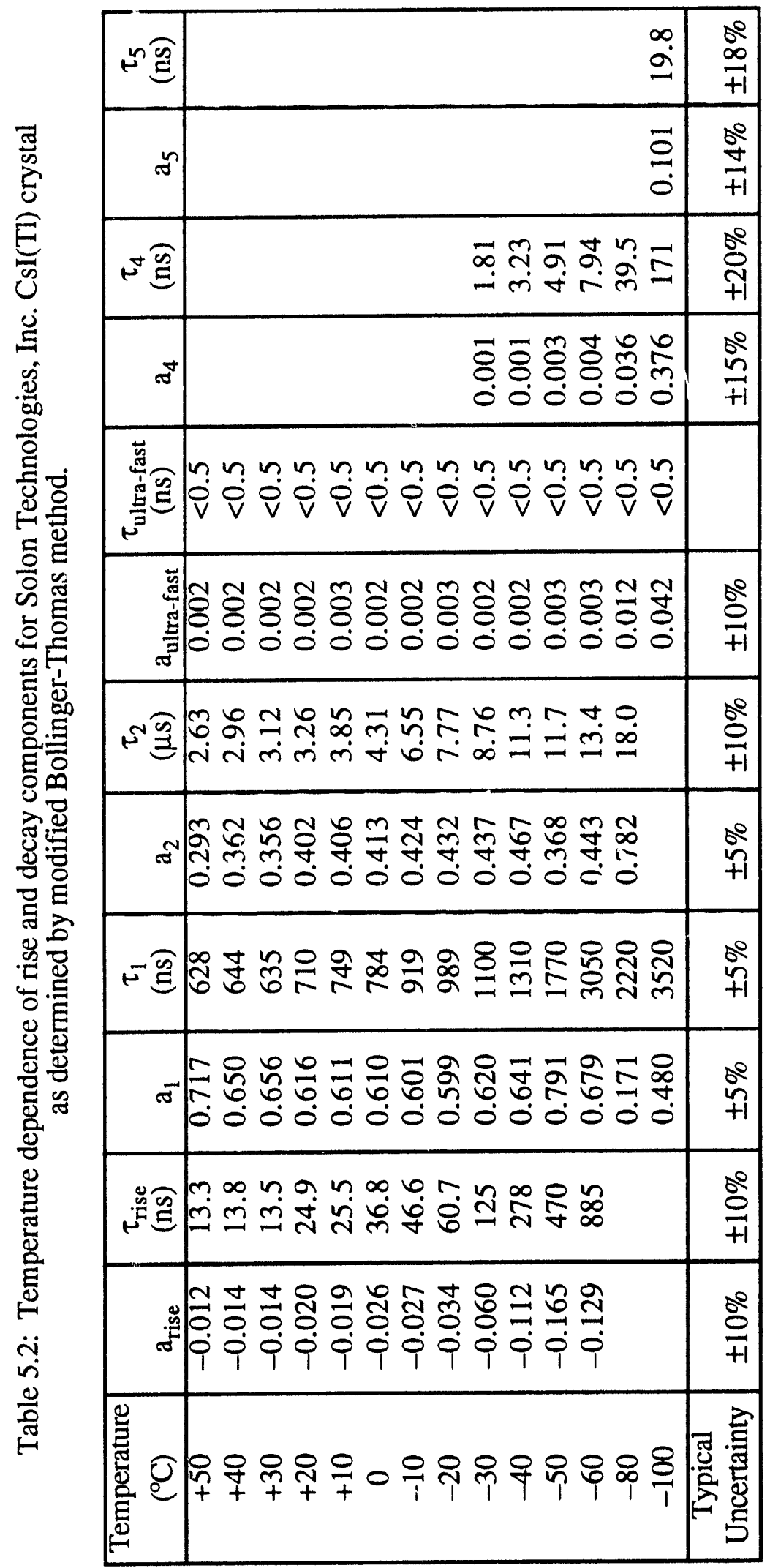


resulted in typical $\chi^{2}$ per degree of freedom values between 1.0 and 1.2 for both the long and short TAC range data. Fitting program output files for every crystal, temperature, and TAC range represented in Tables 5.1 and 5.2 have been compiled in Appendix D. The output files contain the fit parameter values, the uncertainty for each parameter that was determined by the program, background count rate, the fixed delay time, and the $\chi^{2}$ for the fit. Additionally, a table of the $\chi^{2}$ per degree of freedom values for each fit appears in Appendix D.

Even though the long decay component yields $35-45 \%$ of the photons, its initial intensity, $\mathrm{I}_{2}$, is about an order of magnitude lower than the initial intensity of the main component, $I_{1}$, and typically no more than a few times the background (chance coincidence) count rate. Accurately resolving $\tau_{2}$ when using the MBT method thus becomes strongly dependent on the knowledge of the flat background. Since we have observed that $\mathrm{I}_{2}$ decreases with decreasing temperature below $-20^{\circ} \mathrm{C}$ while the background remains nearly constant, accurately determining the background becomes even more important with decreasing temperature. The initial channels of the timing spectra seen in Fig. 5.3 represent the fixed delay introduced into the stop channel prior to the TAC and are typically used to determine the background count rate. Unfortunately, the fixed delays used during the 20 and $50 \mu \mathrm{s}$ TAC range data acquisition resulted in only about 10 to 20 channels before the start to the scintillation pulse. The poor statistical accuracy of determining the background count rate from these channels resulted in large uncertainties in the estimates of the $a_{j}$ 's and $\tau_{j}$ 's. However, by acquiring data with a $20 \mu \mathrm{s}$ TAC range in $16.64 \mathrm{~ns}$ bins for $\mathrm{T}>-20^{\circ} \mathrm{C}$ and a $50 \mu \mathrm{s}$ TAC range in $10.35 \mathrm{~ns}$ bins for $\mathrm{T} \leq-20^{\circ} \mathrm{C}$, the last hundred or so bins of most spectra were approximately down to background level. Consequently, to make the most accurate estimate of the background an additional iterative procedure was implemented, using the last set of bins. During the initial fit, when the initial best estimates of the "longer" decay components were being determined, the background count rate was allowed to vary along with the other parameters. Subsequently, 
the initial best estimates of $\tau_{2}$ and the background count rate were used to fit only the last ten to twenty microseconds of the timing spectrum (where it was assumed that the contribution from $\tau_{1}$ was negligible, since $t>10 \tau_{1}$ ). The resulting background count rate was then held constant while the entire spectrum was fit. The best estimate of $\tau_{2}$ was then held constant while fitting the last ten to twenty microseconds of the spectrum. The background count rate was thus iteratively determined. Once ihis iterative process converged, typically after two iterations, the previously described iterative process was resumed while determining a new best estimate of the background count rate for each new best estimate of $\tau_{2}$.

Deich, et al. [DEI89] and Aluker, et al. [ALU88] have also observed the ultra-fast

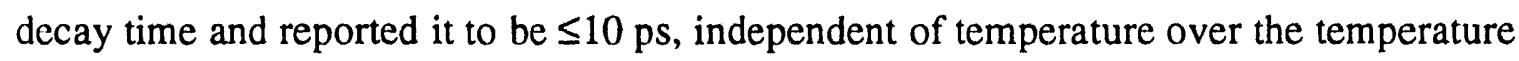
range of 80-400 K, emitted with a broad emission band peaking at about $620 \mathrm{~nm}$, and independent of crystal defects, impurities, and activators. They have attributed this component to intraband luminescence induced by a "hot" charge carrier, as discussed in Section 1.1.4. Since this ultra-fast component is emitted in a time frame much shorter that the timing resolution of the electronics, it is observed as an impulse of light. However, the fitting, program does not recognize a decay time of $<0.5 \mathrm{~ns}$ as an impulse and continues to try to determine the best estimate of the decay time. Consequently, once it was determined that we were observing this ultra-fast component in the $1 \mu \mathrm{s}$ TAC range data at all temperatures for all crystals, the data were analyzed while holding $\tau_{\text {ultra-fast }}$ constant at 10 ps. The fitting program and the iterative procedure converged more rapidly under these conditions and the resulting temperature dependence of the parameters was more consistent with the expected behavior. Additionally, decay component 4 in Table 5.2 is not resolved by the fitting program for $\mathrm{T}=-30^{\circ} \mathrm{C}$ when the ultra-fast component is not held constant Thus, we believe this procedure results in more accurate estimates of the $a_{j}$ 's and $\tau_{j}$ 's, and the resulting parameters are those reported. 
The rise time of $\mathrm{CsI}(\mathrm{Tl})$ became too long to accurately fit below $-60^{\circ} \mathrm{C}$. However, when the rise time was accurately fit, it was always observed to be associated with $\tau_{1}$, as evidenced by the similarity of the initial intensities, $\mathrm{I}_{1}$ and $\mathrm{I}_{\text {rise }}$. Similarly, below $-60^{\circ} \mathrm{C}$ the ability to accurately fit the long decay time is diminished because of signal-to-noise and the decay time approaching the limit of the TAC range. The fitting program requires that the sum of the $a_{j}$ 's always be unity, thus when any of the rise or decay components are not resolved that fraction is removed from the sum. Consequently, when $\tau_{\text {rise }}$ and/or $\tau_{2}$ become unresolvable $\left(\mathrm{T}=-80\right.$ and $-100^{\circ} \mathrm{C}$ in Table 5.2), the $\mathrm{a}_{\mathrm{j}}$ 's no longer accurately estimate the fraction of the total light being emitted by the $\mathrm{j}^{\text {th }}$ process.

The presence of two different decay times is consistent with the observations of Shamovskii and Shushkanov [SHA69]; they noted that two different thallium luminescence centers will be present in $\mathrm{CsI}(\mathrm{Tl})$, as discussed in Section 1.1.1. Furthermore, the association of $\tau_{\text {rise }}$ and $\tau_{1}$ and the absence of a rise time for $\tau_{2}$ suggests that each of the $\mathrm{Tl}^{+}$ centers trap different types of holes. The rise time is probably due to the migration of $\mathrm{V}_{\mathrm{k}}$ centers toward $\mathrm{Tl}^{+}$ions, as discussed in Sections 1.1.3 and 5.3.3, while the instantaneous rise is probably due to holes being trapped as $\mathrm{Tl}^{++}$at $\mathrm{Tl}^{+}$sites, as described in Section 1.1.1.

\subsubsection{Shaped Square Wave Method Results}

To confirm that all four $\operatorname{CsI}(\mathrm{Tl})$ crystals would yield similar $\tau_{1}, \tau_{2}$, and $\mathrm{q}_{2} / \mathrm{q}_{1}$ results from the SSW method, the photopeak centroids for all of the crystals were recorded at room temperature for all amplifier shaping times and are shown in Fig. 5.4. The photopeak centroids for each crystal, taken from Fig. 4.7, were normalized to that crystal's $12 \mu$ s centroid. It is observed that there is less than $3 \%$ deviation in the values for all crystals at each shaping time. Thus, the SSW method will result in very similar $\tau_{1}, \tau_{2}$, and $\mathrm{q}_{2} / \mathrm{q}_{1}$ values for all crystals. The STI crystal was used to determine the temperature dependence of these parameters. 


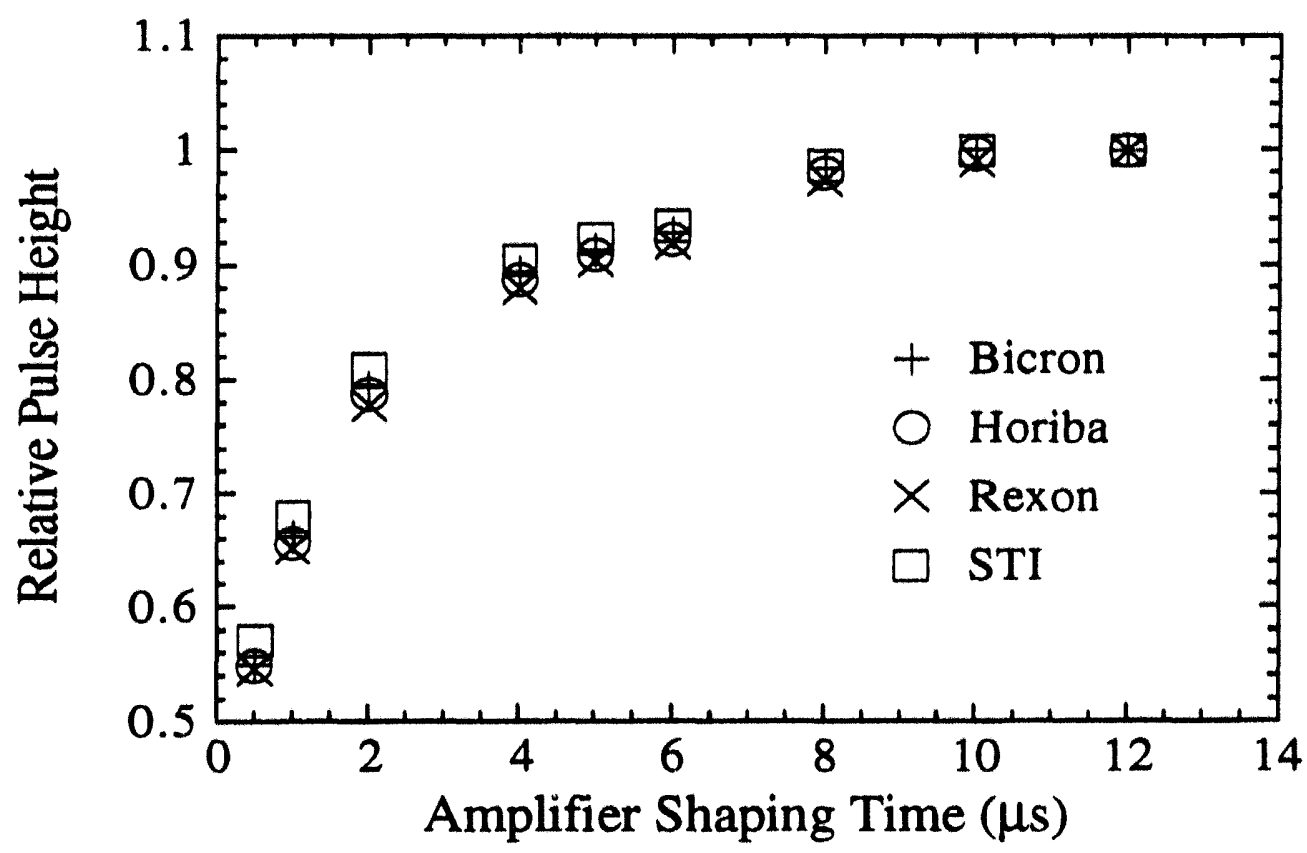

Figure 5.4: Amplifier shaping time dependence of C.SI(Tl) pulse height for crystals from Bicron Corporation, Horiba Crystal Products, Rexon Components, Inc., and Solon Technologies, Inc. Normalized to $12 \mu \mathrm{s}$ pulse height of each crystal.

The STI crystal photopeak centroids, recorded at three different temperatures along with the associated SSW method calibration pulse peak centroids, were shown in Fig. 4.6. These data are typical of that acquired and the associated SSW results at $10^{\circ} \mathrm{C}$ increments over the temperature range of -60 to $+30^{\circ} \mathrm{C}$. When the calibration pulse peak centroids best matched the photopeak centroids at every shaping time constant, the potentiometer settings were recorded and converted to the appropriate decay time $n$ s sharge ratio according to their calibrations. The resulting decay times and charge ratios are shown in Table 5.3. Above $+30^{\circ} \mathrm{C}$, accurate estimates of the photopeak centroids at all amplifier shaping time constants were not achievable due to the increased photodiode dark current that manifests itself as electronic noise during pulse processing. Below $-60^{\circ} \mathrm{C}, \tau_{2}$ becomes longer than the longest amplifier shaping constant and the photopeak centroids for the shorter shaping times begin to become obscured by the noise shoulder. Consequently, for $\mathrm{T}>+30^{\circ} \mathrm{C}$ and $\mathrm{T}<-60^{\circ} \mathrm{C}$ the SSW method could not be used. We have estimated the uncertainty of determining $\tau_{1}, \tau_{2}$, and $q_{2} / q_{1}$ by the SSW method to be $\pm 5 \%$ of the reported value. This 
Table 5.3: Temperature dependence of primary decay time constants and charge contribution ratio for Solon Technologies, Inc. CsI(Tl) crystal as determined by the shaped square wave method.*

\begin{tabular}{|c|c|c|c|}
\hline $\begin{array}{c}\text { Temperature } \\
\left({ }^{\circ} \mathrm{C}\right)\end{array}$ & $\begin{array}{c}\tau_{1} \\
(\mathrm{~ns})\end{array}$ & $\begin{array}{c}\tau_{2} \\
(\mu \mathrm{s})\end{array}$ & $\mathrm{q}_{2} / \mathrm{q}_{1}$ \\
\hline+30 & 809 & 5.00 & 0.680 \\
+20 & 832 & 5.50 & 0.760 \\
+10 & 956 & 6.85 & 0.742 \\
0 & 1040 & 7.97 & 0.760 \\
-10 & 1060 & 8.58 & 0.865 \\
-20 & 1100 & 9.47 & 0.927 \\
-30 & 1150 & 10.40 & 1.01 \\
-40 & 1190 & 11.20 & 1.09 \\
-50 & 1240 & 12.20 & 1.17 \\
-60 & 1370 & 12.90 & 1.19 \\
\hline
\end{tabular}

*Estimated uncertainty of $\pm 5 \%$ on all values.

uncertainty is due to the uncertainty of the potentiometer calibrations, which we have assumed dominates all other sources of random uncertainty.

\subsubsection{Comparison of MBT and SSW Results}

A comparison of the best estimates of $\tau_{\text {rise }}, \tau_{1}$, and $\tau_{2}$ from the modified BollingerThomas method and SSW method is shown in Fig. 5.5. As previously mentioned, the rise time was held constant for all of the SSW method calibrations hecause the SSW method is not sensitive to characteristic times shorter than the shortest available amplifier shaping time (250 ps for our measurements). Estimated uncertainties are smaller than the symbols used in Fig. 5.5. The rise and decay times are plotted versus $1 / \mathrm{T}$ to confirm the behavior predicted by Eqn. (5.1). Activation energies of $0.23,0.063$, and $0.090 \mathrm{eV}$ for $\tau_{\text {rise }}, \tau_{1}$, and $\tau_{2}$, respectively, were determined by fitting the modified Bollinger-Thomas results to Eqn. (5.1), while values of 0.030 and $0.058 \mathrm{eV}$ for $\tau_{1}$ and $\tau_{2}$, respectively, were determined by fitting the SSW method results. The resulting rise time activation energy, $0.23 \mathrm{eV}$, corresponds closely to the activation energy associated with the $90^{\circ}$-reorientation of $\mathrm{V}_{\mathrm{k}}$ centers in pure CsI, reported by Pellaux [PEL76] to he $0.198 \mathrm{eV}$, and to the rise time 


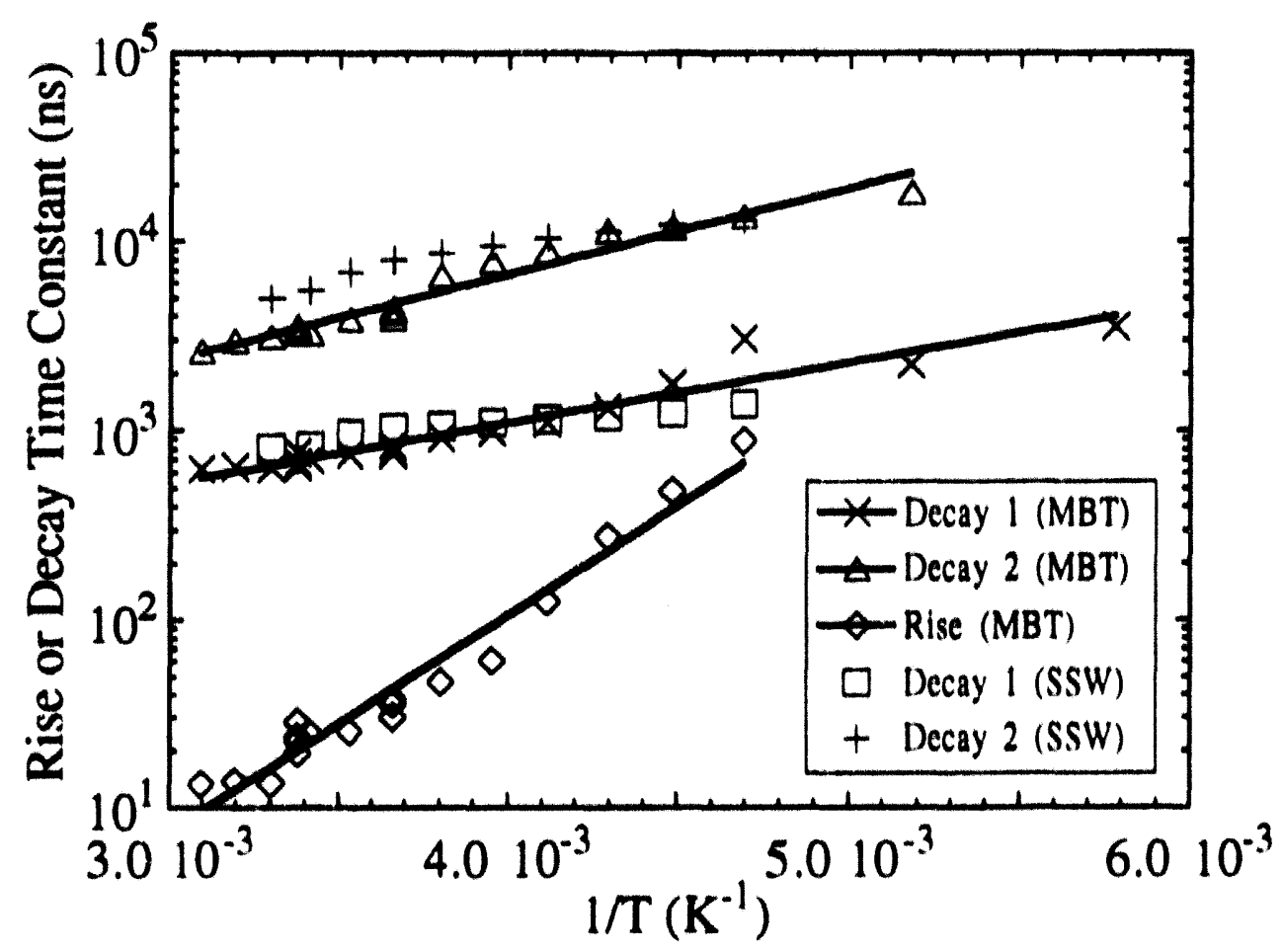

Figure 5.5: Comparison of the modified Bollinger-Thomas (MBT) method and shaped square wave (SSW) method rise and decay time constant results for CsI(TI),

activation energy reported by Aluker, et al. [ALU86] of $0.2 \mathrm{eV}$. Consequently, our results seem to confirm the prediction by Aluker, et al. [ALU86] that the rise time can be attributed to the migration of $\mathrm{V}_{\mathrm{k}}$ centers toward thallium luminescence centers via $90^{\circ}$-reorientation. If only the SSW results for $\mathrm{T} Z-10^{\circ} \mathrm{C}$ are fit to Eqn. (5.1), then the activation energies for $\tau_{1}$ and $\tau_{2}$ are 0.052 and $0.099 \mathrm{eV}$, respectively. These activation energies agree much hetter with the MBT values than when fitting for all temperatures. Therefore, we believe that assuming that variations in the rise time do not affect the SSW method is reasonable for $\mathrm{T} Z-10^{\circ} \mathrm{C}$. Thus, the difference between the SSW and MBT results above $-10^{\circ} \mathrm{C}$ is prohably due to a systematic error in the calibration of the $\tau_{1}$ and $\tau_{2}$ potentiometers. However, the rise time variations are causing $\tau_{1}$ and $\tau_{2}$ to he underestimated below $-10^{\circ} \mathrm{C}$ after accounting for the systematic error. It is also possible that $\tau_{2}$ becomes too long to be accurately determined by the SSW method below $-10^{\circ} \mathrm{C}$. Furthermore, we believe that the activation energies that were determined by fitting the MBT results are more accurate than 


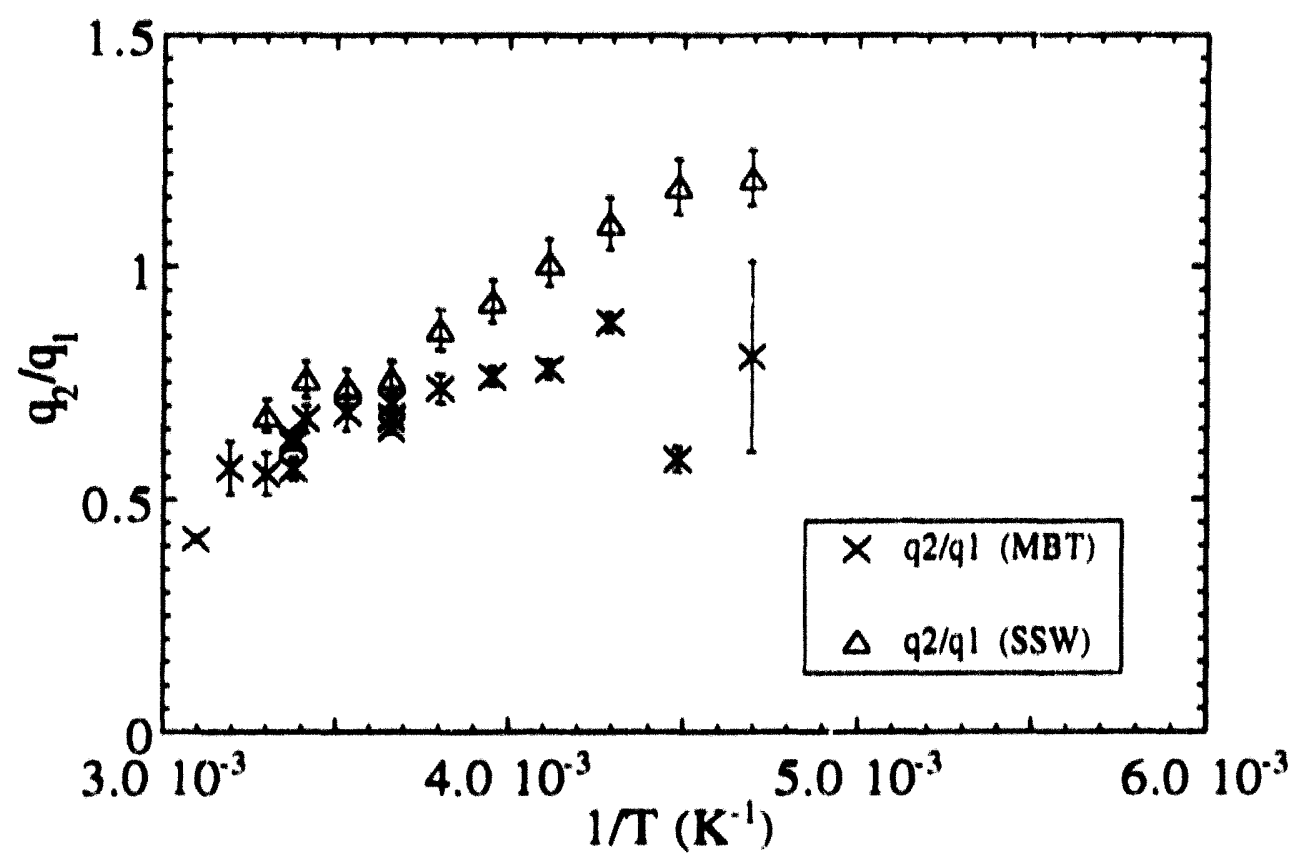

Figure 5.6: Comparison of the modified Bollinger-Thomas (MBT) method and shaped square wave (SSW) method charge contribution ratio results for Csl(TI).

from the SSW results.

The SSW method yields a ratio of the charge contributions from the two primary components to the total pulse, $q_{2} / q_{1}$. This charge ratio is equivalent to the ratio of the integrated luminescence of the two primary components, $a_{2} /\left(a_{1}+a_{\text {rise }}\right)$, from the modified Bollinger-Thomas method. A comparison of the charge ratio results from the MBT and SSW methods is shown in Fig. 5.6. When the rise time could not be accurately fit by the MBT method $\left.(T<-60)^{\circ} \mathrm{C}\right)$, the charge ratio could not be calculated. Since the SSW charge ratios are larger than the MBT results we believe that there was a systematic error in the calibration of the potentiometer used by the SSW method. Both methods appear to yield a similar charge ratio temperature dependence for $\mathrm{TZ}-10^{\circ} \mathrm{C}$, but diverge below $-10^{\circ} \mathrm{C}$. To successfully apply the SSW method, the integrated charge must be nearly equal for the $\mathrm{CsI}(\mathrm{Tl})$ and calihration pul:es for all amplifier shaping times. Furthermore, if the rise and decay times are underestimated by the SSW method below $-10^{\circ} \mathrm{C}$, as concluded above, the resulting charge ratio will be an overestimate. Fig. 5.7 demonstrates the difference in the 


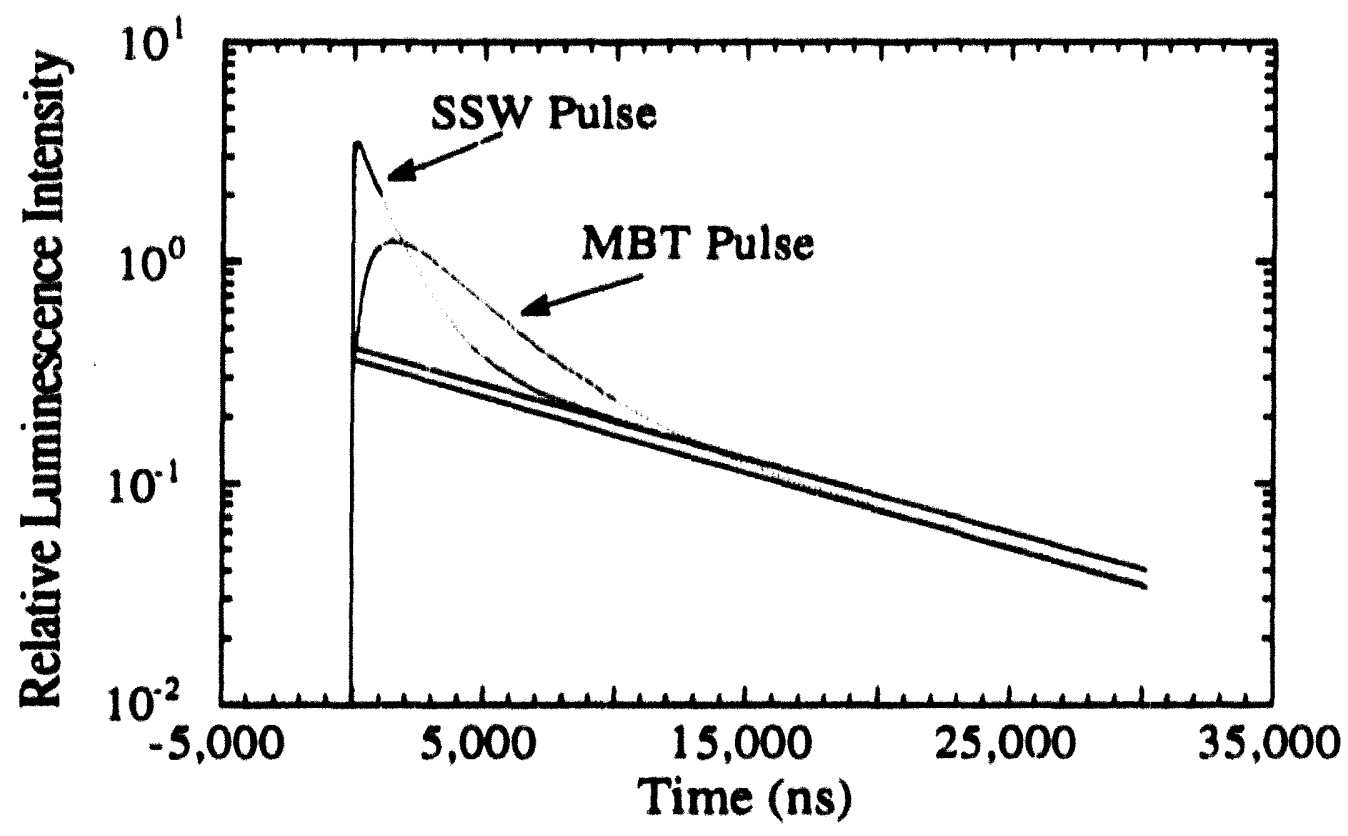

Figure 5.7: Comparison of the basic Csl(TI) pulse shape (rise and two primary decay components) from modified Bollinger-Thomas (MBT) and shaped square wave (SSW) results for Solon Technologies, Inc. crystal at $-60^{\circ} \mathrm{C}$.

charge ratio results by showing the basic $\mathrm{CsI}(\mathrm{Tl})$ pulse shape (the rise and two primary decay times) as determined by both methods at -60$)^{\circ} \mathrm{C}$, where the lines distinguish between luminescence modes 1 and 2 for each method. The areas above and below the line are equivalent to $q_{1}$ and $q_{2}$, respectively. From Tables 5.2 and 5.3, we see that the total relative integrated charge values (or areas in the figure) for both methods are about $q_{1}^{S S W}=0.457, q_{2}^{S S W}=0.543, q_{1}^{M B T}=0.550, q_{2}^{M B T}=0.443$. Consequently, we believe the trend shown by the MBT data in Fig. 5.6 most accurately represents the behavior of the temperature dependence of the charge ratio. It is not obvious from the figure that the integrated charge from the SSW method for short amplifier shaping times will be similar to that from the CsI(Tl) pulse. However, when the ultra-fast component and decay component 4 from the MBT results (not represented in Fig. 5.7) are considered, some of the disparity is accounted for. In this illustration, we have not corrected the SSW results for the proposed systematic error in the potentiometer calibrations. 
Although the SSW method yields some estimates of the primary decay time constants and charge ratio that are outside the uncertaintics of the modified BollingerThomas method results, the SSW method results are reasonable estimates with a possible systematic bias. These systematic uncertainties in the SSW method results are probably due to the rise time component not heing modeled exactly at each temperature and inaccurate potentiometer calibrations. Modeling the rise time at each temperature with the SSW method would increase the activation energies for $\tau_{1}$ and $\tau_{2}$ and would decrease the charge ratios below $-10^{\circ} \mathrm{C}$. A systematic inaccuracy in one of the potentiometer calibrations would shift all of the data for the associated parameter up or down. Even though the results of the SSW method are sensitive to the rise time, the implementation of the calibration (matching photopeak and calihration peak centroids) is not. Thus, the rise time would have to he known at each temperature before the SSW method could he implemented. Consequently, we helieve that the MBT results reported are a hetter representation of the temporal luminescence intensity of Csl(TI). 


\section{CHAPTER VI \\ THERMOLUMINESCENCE}

As mentioned in Chapter I, CsI(TI) has been previously reported to be thermoluminescent in the temperature range of -100 to $+50^{\circ} \mathrm{C}$ [ORA84, GUT74]. The cmission of thermoluminescence is the result of an increase in crystal temperature providing the activation energy necessary to liberate a trapped state. The trapped state that is liberated can be a trapped hole, a trapped electron, or a trapped excited state (e.g., $\left.\left.(\mathrm{T})^{+}\right)^{*}\right)$ and must recombine at a luminescence center after being untrapped to be considered thermoluminescence. The presence of thermoluminescence is observed after free electrons and holes are created in a scintillator by ionizing radiation (electrons and holes created by photons in the absorption band of a scintillator are typically excitons, and thus not free) and the crystal subsequently warmed. For CsI(TI), Gutan, et al. [GUT74] have also noted the emission of inertial luminescence in temperature ranges of high thermoluminescence yield. Inertial luminescence is the result of the same process as thermoluminescence, except that the activation energy is provided by the transfer of kinetic energy from a "high" energy electron (created by a gamma-ray interaction) to a trapped state. While thermoluminescence appears during or immediately after a change in crystal temperature, inertial luminescence will be of concern primarily at thermal equilibrium.

Although thermoluminescence emissions are not typically considered when evaluating the characteristics of a scintillator, thermoluminescence can affect both current and pulse mode measurements. For current mode applications, thermoltiminescence will directly lead to erroneous inferences about the incident heam of photons or charged particles due to the increased light yield from the scintillator. For the current mode 
measurements with temperature regulation $C$ used in Chapter IV to determine the scintillation yield, the crystal temperature was always decreasing. In this manner, thermoluminescence was eliminated from these measurements. The effect of thermoluminescence on pulse mode measurements is more subtle. Since thermoluminescence photons have no correlation in time with any scintillation event and have typical decay times much longer than any scintillation decay time, these photons will appear us a noisy de signal to the photodetector relative to scintillation events (similar to dark current). Also, typical pulse mode methods will have much Inwer counting rates than current mode methods, and thus will not be as susceptible to inertial luminescence hecause of the decreased energy deposited in the crystal per unit time. Thus. thermoluminescence will appear as a de-offset for pulse mode methods that integrate the charge created in the photodetector hy a gamma-ray interaction in the crystal. A coupling capacitor can casily he used to decouple the ac (signal) and de (dark current and thermoluminescence) components. However, the increased level of the de component due to thermoluminescence will degrade signal-10-noise. The degradation of energy resolution due to the increased de component will only be significant during periods of high thermoluminescence emissions, during or immediately after a change in crystal temperature. Consequently, thermoluminescence should not degrade performance when detectors are operated at thermal equilibrium. The photopeak centroids used to perform the shaped square wave method were all acquired ahout an hour after the detector temperature was stahilized and also at low count rates, and thus we helieve that both therrnoluminescence and inertial luminescence did not affect our measurements. For pulse mode methods where single photons (or photoelectrons) are heing counted, thermoluminescence and inertial luminescence will appear as an increased hackground (or (lark) rate. A coincidence method that uses single photons to generate timing signals, such as the modified Bollinger-Thomas (MBT) method, will see this manifestation of thermoluminescence as an increased chance coincidence rate. Thus, the timing spectra 
acquired using the MBT method may be more difficult to fit due to the presence of thermoluminescence or inertial luminescence, and resulting rise and decay component parameter values will have larger uncertainties. Methods that rely on recording the count rate of single photons, like the method used to measure the Csl(Tl) emission spectrum in Chapter III, will be susceptible to thermoluminescence and inertial luminescence due to the increased dark rate. Consequently, we have measured the thermoluminescence emissions of the four different $\mathrm{CsI}(\mathrm{Tl})$ crystals over the range of -1100 to $+50^{\circ} \mathrm{C}$.

\subsection{Thermoluminescence Experimental Methods}

As mentioned in Chapter IV, the current mode method was found io yield erroneous results when used with temperature regulation method $A$ (Fig. 3.2) to determine the scintillation yield temperature dependence. To illustrate these difficulties, the Solon Technologies, Inc. CsI(TI) crystal scintillation yield was measured using the current mode method temperature regulation method $A$ fron: -100 to +50$)^{\circ} \mathrm{C}$. The measurements were performed by first heating the crystal to $+50^{\circ} \mathrm{C}$, recording the PMT output current, then decreasing the temperature in $10^{\circ} \mathrm{C}$ increments while continuously recording the PMT output current. The crystal temperature was held at each $10^{\circ} \mathrm{C}$ increment for at least half of on hour to assure that thermal equilibrium was achieved.

To determine the temperatures and magnitudes of the peak thermoluminescence umissions, the "glow curves" of each CsI(Tl) crystal were measured. Data acquisition was achieved using the sume detector geometry and electronics schematic as the current mode measurements of the scintillation yield (Figs, 5.2 and 5.3, respectively). After the crystals were brought to thermal equilibrium at $-1(1))^{\circ} \mathrm{C}$ at the conclusion of the current mode method scintillation yield measurements, the liquid nitrogen flow was turned off and the heating element turned on, temperature regulation method $D$ (Fig. 6.1). The crystal was subsequently heated at a rate of about $5^{\circ} \mathrm{C}$ per minute while the PMT output current was recorded. The scintillation yield measurements required the ${ }^{68} \mathrm{Ge}$ source be located inside 


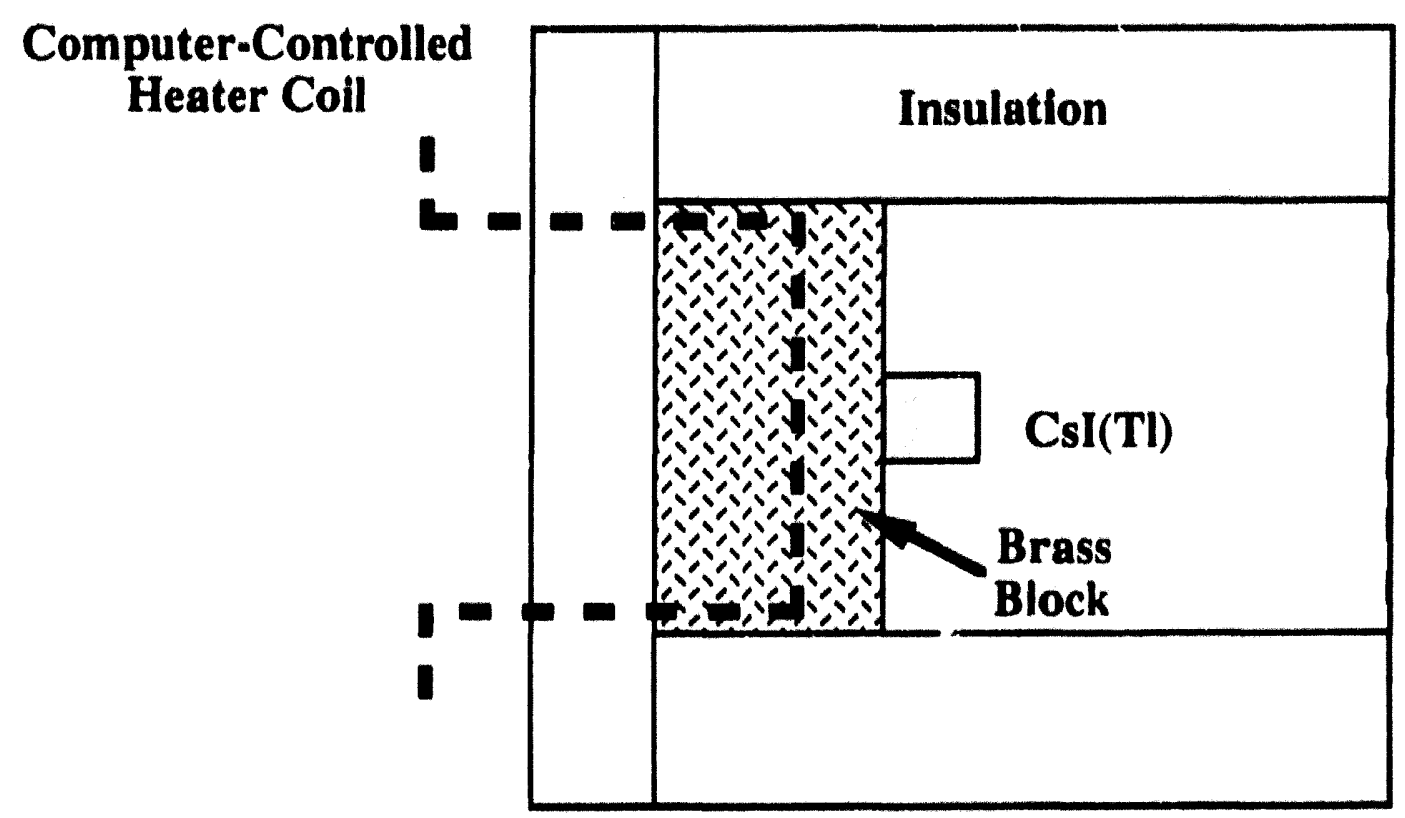

Figure 6.1: Schematic of temperature regulation method $D$.

the temperature-controlled enclosure to produce as high a PMT output current as possible. Consequently, the source could not be removed betore the thermoluminescence measurements. Due to this continuous irradiation it was necessary to subtract the gammaray excited scintillation yield (determined using temperature regulation method $C$ ) from the recorded data to arrive at thermuluminescence yield.

To determine the wavelengths of the thermoluminescence emissions, the electronics for the emission spectrum measurements (Fig. 3.1) were used with temperature regulation method $A$ and the STI CsI(Tl) crystal. The crystal temperature was increased abruptly $10^{\circ} \mathrm{C}$ in a temperature range where high thermoluminescence yield was observed with the monochromator passing the $400 \mathrm{~nm}$ band light, and at a different time the $560 \mathrm{~nm}$ band light, while the PMT output current was monitored.

\subsection{Thermoluminescence Results}

When using temperature regulation method $A$, the PMT output current for the STI crystal was observed to pass through a minimum then rise to its equilibrium value when 


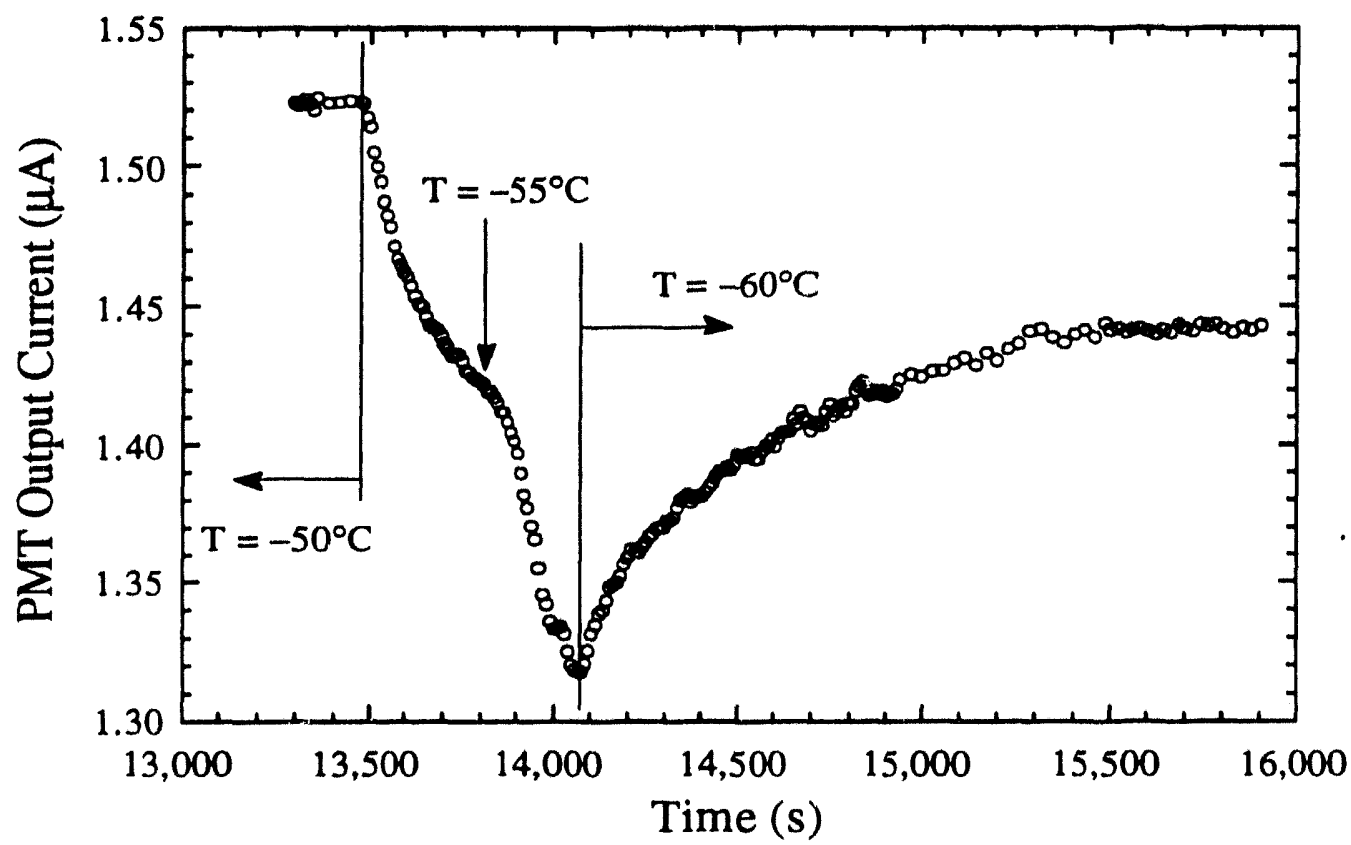

Figure 6.2: Current mode method results in temperature range of high thermoluminescence yield for temperature regulation method $A$. Data taken while changing from thermal equilibrium at $-50^{\circ} \mathrm{C}$ to thermal equilibrium at $-60^{\circ} \mathrm{C}$ for Solon Technologies, Inc. CsI(Tl) crystal.

decreasing the crystal temperature $10^{\circ} \mathrm{C}$ from one state of thermal equilibrium to another between -30 and $-80^{\circ} \mathrm{C}$. Fig. 6.2 illustrates this behavior for a change of crystal temperature from -50 to $-60^{\circ} \mathrm{C}$. For temperature regulation method $A$, the heater coil cycles on and off to counter the constant flow of liquid nitrogen at thermal equilibrium. As seen in the figure, the temperature controller was programmed to decrease as quickly as possible to $-60^{\circ} \mathrm{C}$, starting at $t \approx 13,500$ seconds. Thus, the heater coil was off until the crystal reached about $-60^{\circ} \mathrm{C}$, when it started to cycle on and off again to stabilize the temperature. The figure shows that while the heater coil was off, states were being trapped, but once the coil began to cycle again some of the trapped states were liberated with resulting luminescence. If the thermocouple reading was significantly lagging the true crystal temperature, these results might be interpreted as the crystal temperature decreasing below $-60^{\circ} \mathrm{C}$ before the heater coil began to cycle, and subsequently increasing back up to $-60^{\circ} \mathrm{C}$. Thus, the thermal equilibrium PMT output current would be representative of the 


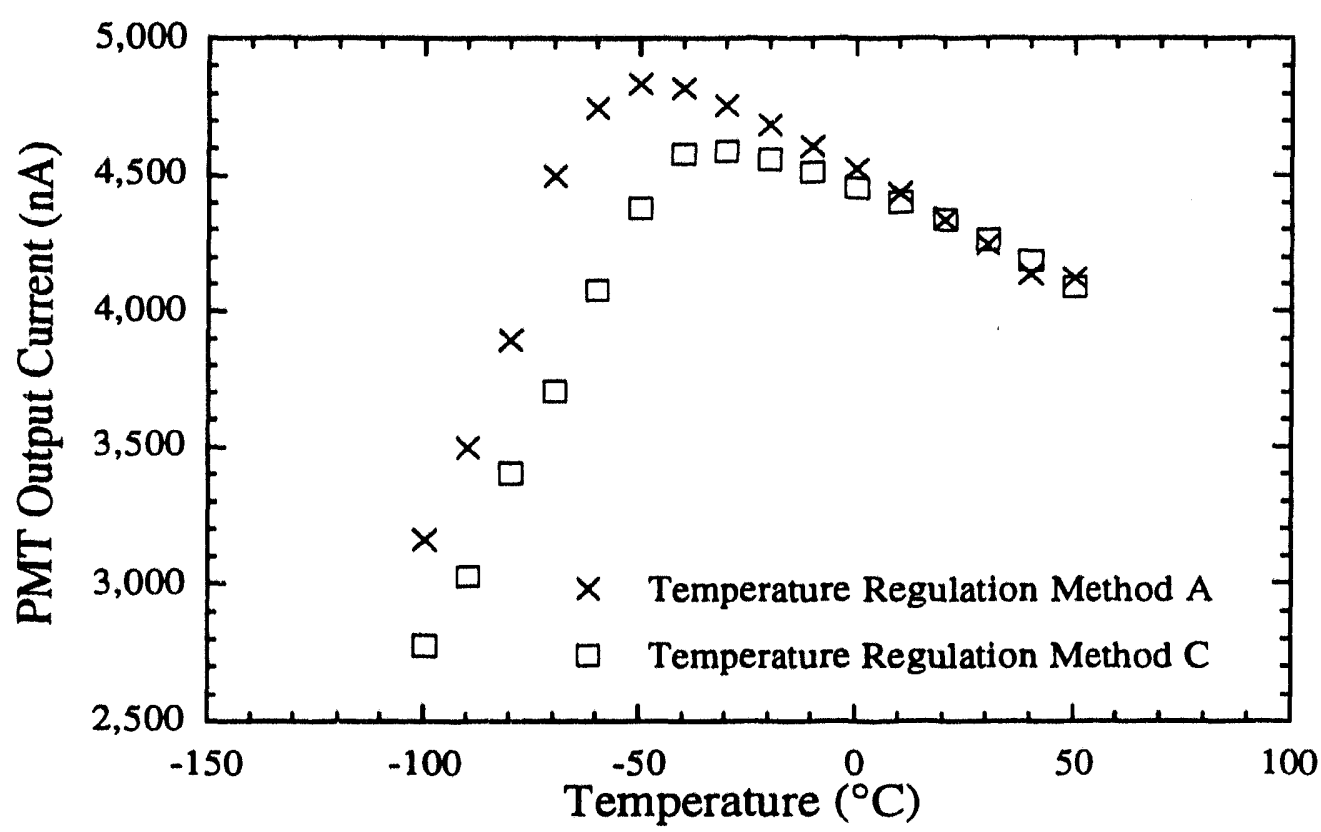

Figure 6.3: Difference in current mode method scintillation yield results for temperature regulation methods $A$ and $C$ for Solon Technologies, Inc. CsI(Tl) crystal, normalized at $+20^{\circ} \mathrm{C}$.

scintillation yield. However, when the scintillation yields inferred from the current mode method with temperature regulation methods $A$ and $C$ are compared (Fig. 6.3), we see that the thermal equilibrium results for temperature regulation method $A$ over-estimate the scintillation yield for $\mathrm{T}<25^{\circ} \mathrm{C}$. Thus, we conclude that inaccurate temperature readings are not the cause of the behavior seen in Fig. 6.2. We believe that this behavior suggests that small temperature variations and/or thermal gradients across the crystal due to the nature of temperature regulation method $A$ were causing some equilibrium thermoluminescence release with a possible contribution from inertial luminescence.

As mentioned, the crystal was continuously irradiated during the thermoluminescence measurements, and thus it was necessary to subtract the scintillation yield of the crystal from the thermoluminescence data. Fig. 6.4 shows the data acquired using the current mode method while cooling and warming the crystal (temperature regulation methods $C$ and $D$, respectively) to determine the scintillation yield and thermoluminescence emissions, respectively, for the STI crystal. Thermoluminescence is 


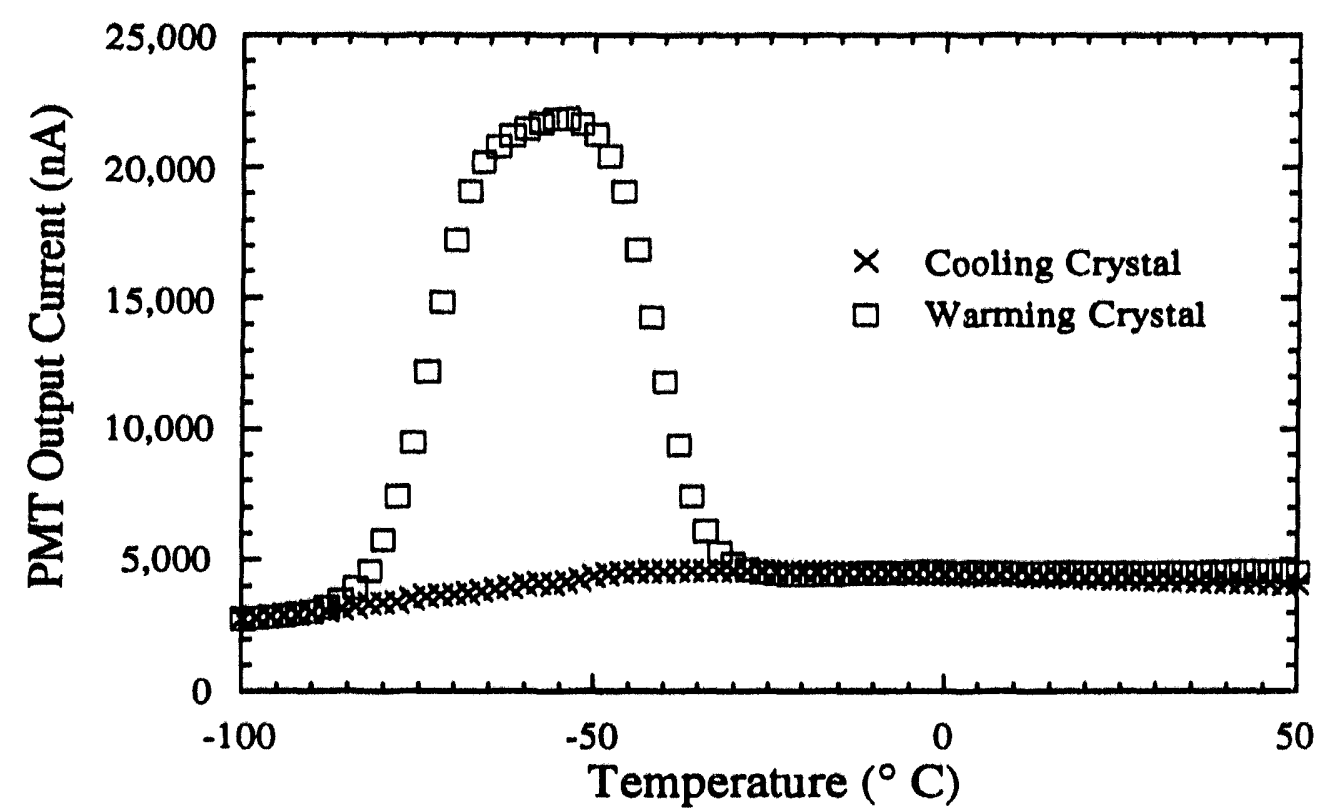

Figure 6.4: Difference in current mode method results observed when cooling and warming (temperature regulation methods $C$ and $D$, respectively) Solon Technologies, Inc. CsI(Tl) crystal.

present for the data taken while the crystal was being warmed that is above the scintillation yield data. The resulting thermoluminescence "glow curves" for the four different crystals are shown in Fig. 6.5. The thermoluminescence yield for each crystal has been normalized to its room temperature scintillation yield. It is observed that all of the crystals have thermoluminescence emission peaks at about $-65^{\circ} \mathrm{C}$, the STI crystal either has peaks at -65 and $-55^{\circ} \mathrm{C}$ or one very broad peak, the Bicron, Horiba, and Rexon crystals all have peaks at about -90 and $+20^{\circ} \mathrm{C}$, and the Bicron crystal has an additional peak at about $-40^{\circ} \mathrm{C}$. It is not known why these significant crystal-to-crystal differences are observed, but using CsI(Tl) crystals in temperature ranges of large thermoluminescence yield may lead to erroneous results.

To determine the wavelengths of the thermoluminescence emissions, the $400 \mathrm{~nm}$ band and the $560 \mathrm{~nm}$ band light were passed through the monochromator at different times, while the STI crystal temperature was quickly changed from -60 to $-50^{\circ} \mathrm{C}$. The results are 


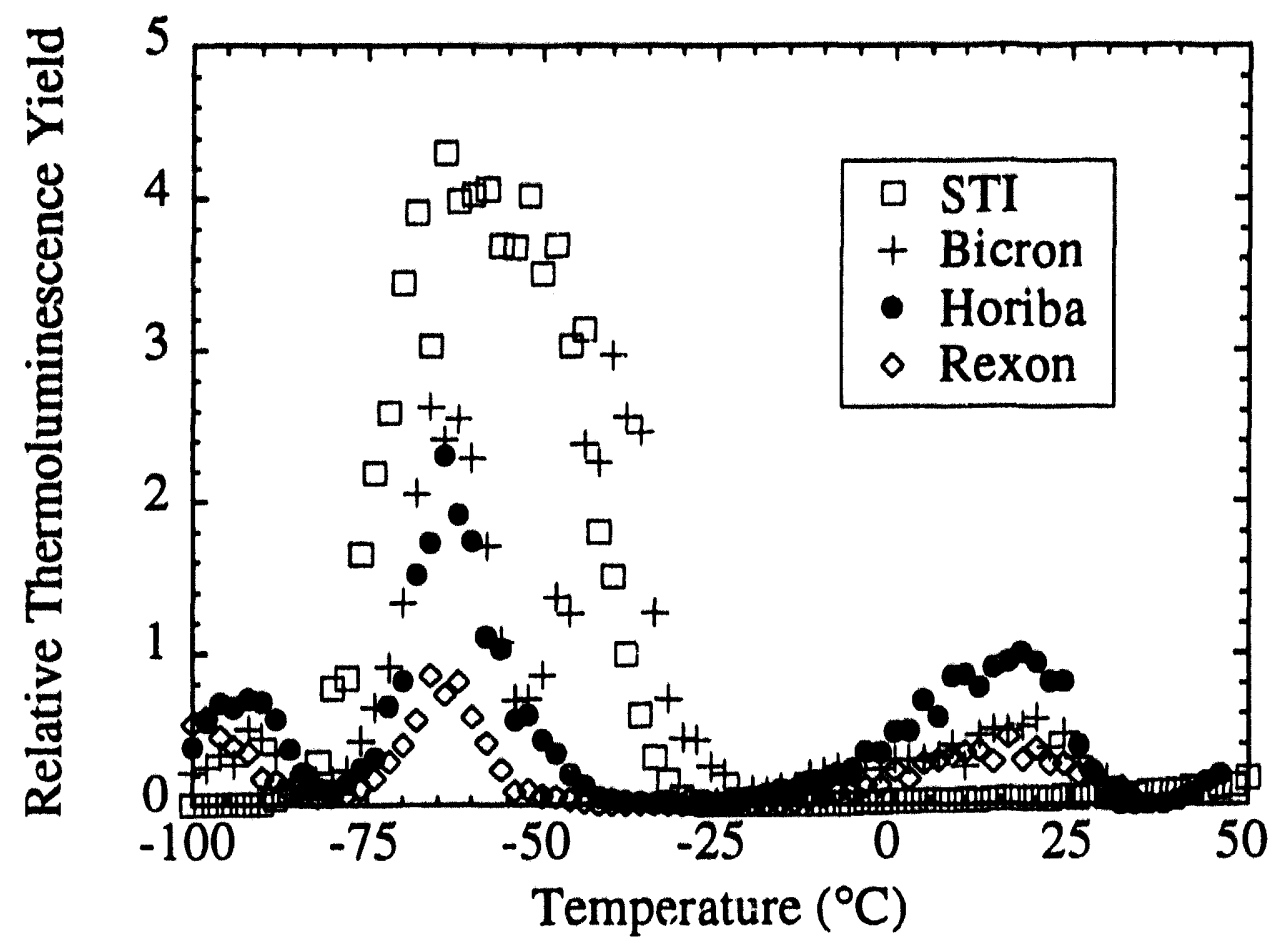

Figure 6.5: CsI(Tl) thermoluminescence "glow curves" for crystals from Solon Technologies, Inc., Bicron Corporation, Horiba Crystal Products, and Rexon Components, Inc., measured by the current mode method, normalized to room temperature scintillation yield of each crystal.

shown in Fig. 6.6, where we see that $\mathrm{CsI}(\mathrm{Tl})$ thermoluminescence is emitted in both bands and decays within ten minutes of the initial temperature change. Consequently, thermoluminescence and/or inertial luminescence emissions will affect the count rate of the emission spectra data, but we believe that the overall emission spectrum shape will not be affected due to the emission in both bands.

The observation of thermoluminescence at $-65^{\circ} \mathrm{C}$ from all of the crystals can be explained by the storage of energy at thallium luminescence centers and at $F$ centers. As described in Section 1.1.1, Van Sciver [VAN56] reported that some transitions of the excited $\mathrm{Tl}^{+}$state to the ground state are forbidden. These trapped states must be thermally excited to a state that does not have a forbidden transition to the ground state for luminescence to be observed. With decreasing temperature, the thermal excitation will become less probable and the energy will be stored until the temperature is increased. 


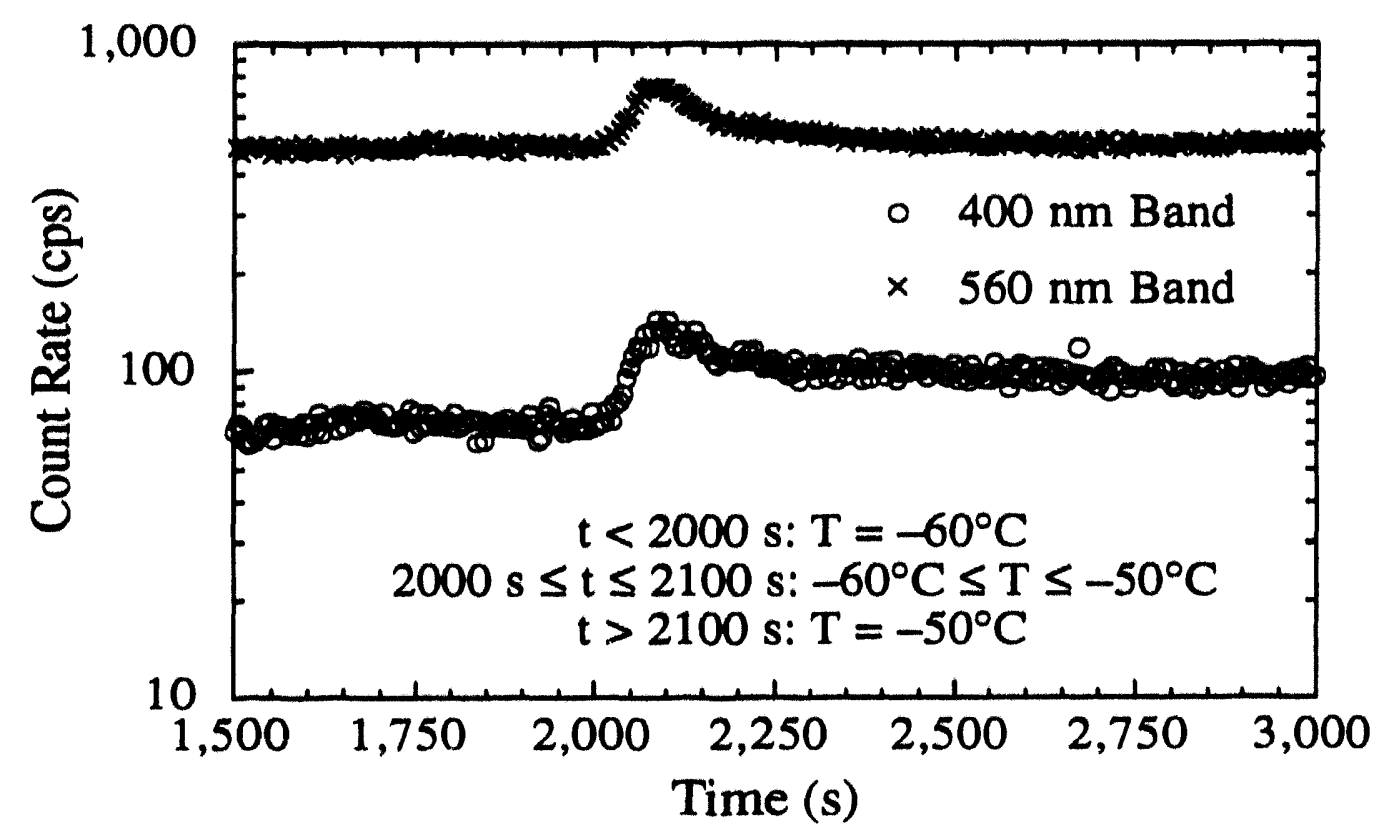

Figure 6.6: Comparison of 400 and $560 \mathrm{~nm}$ band thermoluminescence when quickly changing Solon Technologies, Inc. CsI(Tl) crystal temperature from -60 to $-50^{\circ} \mathrm{C}$.

Additionally, we observed the disappearance of the $400 \mathrm{~nm}$ band in the CsI(Tl) emission spectrum below $-50^{\circ} \mathrm{C}$. Since thermoluminescence was observed to be emitted in both the 400 and $560 \mathrm{~nm}$ bands, we assume that the absence of $40 \mathrm{~nm}$ band light in the emission spectrum below $-50^{\circ} \mathrm{C}$ indicates that this energy 1. being stored. Since the thermoluminescence peak temperatures seen in Fig. 6.5 were not the same for all crystals (except for the $-65^{\circ} \mathrm{C}$ peak), we believe that these peaks are probably due to different impurities in each crystal. 


\section{CHAPTER VII CONCLUSIONS}

The gamma-ray excited emission spectrum, absolute scintillation yield, rise and decay time constants, and thermoluminescence emissions of CsI(TI) have been measured over the temperature range of -100 to $+50^{\circ} \mathrm{C}$. These characteristics were evaluated for CsI(Tl) crystals from four different vendors. Negligible differences were observed in the four different emission spectra at room temperature. The decay times for the four different crystals were found to vary by less than $10 \%$ at $0^{\circ} \mathrm{C}$ and $17 \%$ at room temperature. The crystals were observed to have similar scintillation yield temperature dependence, with yields peaking at temperatures ranging from -35 to $-20^{\circ} \mathrm{C}$. The thermoluminescence "glow curves" were the only characteristic that varied significantly from crystal to crystal. Thermoluminescence emissions were found to peak at about $-65^{\circ} \mathrm{C}$ for all crystals, while peaks at $-90,-40,+20$, and possibly $-55^{\circ} \mathrm{C}$ were observed for some crystals. For the STI crystal, it was found that thermoluminescence and/or inertial luminescence in the range of -80 to $-30^{\circ} \mathrm{C}$ affect both current and pulse mode measure nents. Low count rate applications will suffer less from inertial luminescence since less kinetic energy is deposited in the crystal per unit time. Similar problems should arise for any CsI(Tl) crystal in temperature ranges of large thermoluminescence yield. An important implication of these thermoluminescence findings is that results of applications that require room temperature operation in current mode could be susceptible to temperature fluctuations.

The CsI(Tl) emission spectrum was observed to have emission bands peaking around 400 and $560 \mathrm{~nm}$. These bands are believed to be attributed to the recombination of $\mathrm{F}$ centers at $\mathrm{V}_{\mathrm{k}}$ centers trapped adjacent to a $\mathrm{Tl}^{+}$ion and the recombination of holes and 
electrons at thallium luminescence centers, respectively. Each of these bands may be made up of more than one emission peak, but the spectral resolution of the system used was not sufficient to resolve these peaks. However, our interest was not in the fine structure of the emission spectrum but in the general weighting of wavelengths. The $4(X 0) \mathrm{nm}$ band was observed to disappear between -50 and $-75^{\circ} \mathrm{C}$, while the shape of the $560 \mathrm{~nm}$ band did not change signiffcantly with temperature. Although the emission spectrum measurements for the STI crystal were probably affected by inertial luminescence and/or thermoluminescence in the -80 to $-30^{\circ} \mathrm{C}$ range, it was not observed to affect the shape of the spectrum. Thus, comparing the peak count rate of the emission spectrum or the area under the emission spectrum at different temperatures could lead to erroneous conclusions. However, the emission spectra were normalized to unit area so that the wavelength-averaged quantum efficiency could be calculated, thus only the shape of the emission spectrum was of interest.

The room temperature absolute scintillation yield of CsI(TI) was calculated to be $65.500 \pm 4,100$ photons/MeV by determining the light collection efficiency $(\eta=0.814 \pm 3 \%$ ), the wavelength-averaged quantum efficiency $(\bar{Q}=0.897 \pm 2 \%)$, and the number of charge carriers created in the PD per unit energy deposited $\left(N_{c c} / E_{\gamma}=47,80(13 \%)\right.$. The computer program DETECT was used to model $\eta$ for the detector geometry used, while the CsI(TI) emission spectrum was measured using a monochromator and averaged over the spectral sensitivity of a calibrated PD to determine $\bar{Q}$; the SSW method was used to determine $\mathrm{N}_{\mathrm{cc}}$. The $6.2 \%$ uncertainty of the scintillation yield was determined from the estimated uncertainties of the three parameters and our estimate of the crystal-to-crystal variations $( \pm 3.6 \%)$. The reported absolute scintillation yield is about $17 \%$ larger than had been previously reported. The reason that our yield is larger (and we believe more accurate) is that we have accounted for the ballistic deficit due to the long decay time of CsI(TI) in our charge calibration with the SSW method. To our knowledge, the hallistic deficit correction has not been performed previously. CsI(Tl) has the largest known absolute scintillation 
yield of inorganic scintillating crystals, and thus has the possibility of having the smallest relative fluctuation in the number of charge carriers. Consequently, CsI(TI) has the possibility of having the best energy resolution of known inorganic scintillating crystals. The uniformity of the light collection efficiency across the detector volume will affect the charge carrier statistics and the contribution of electronic noise to the signal affect energy resolution as well, but the scintillation yield will typically be the limiting contribution to energy resolution. Unfortunately, the significant ballistic deficit does not allow the full integration of the yield for present commercially-available amplifiers.

The temperature dependence of the CsI(TI) scintillation yield was measured by the SSW method and an independent current mode method. The two methods produce similar results for $\mathrm{TZ}-10^{\circ} \mathrm{C}$, but the results diverge below this temperature. We believe that the current mode method yields the most accurate representation of the CsI(TI) scintillation yield temperature dependence. Furthermore, we believe that the divergence of the SSW results is due to one or more of the following: a) the CsI(TI) rise time was not modeled accurately at each temperature, b) the assumption of PD quantum efficiency temperature independence was not accurate, or c) the decay times of CsI(TI) becom's too long for the SSW method. From the current mode measurements, the maximum scintillation yield is observed to occur at about $-35^{\circ} \mathrm{C}$ for the STI crystal, approximately $6 \%$ ahove the room temperature yield. The yield is also observed to decrease monotonically above and below the maximum. At -100 and $+50^{\circ} \mathrm{C}$, the yield was measured to be about $64 \%$ and $95 \%$ of the room temperature yield, respectively. The decrease in scintillation yield below $-35^{\circ} \mathrm{C}$ is helieved to he at least partially due to energy heing stored in the crystal as trapped luminescence states. This stored energy is the source of the thermoluminescence light that is released when the crystal is warmed. The peaking of the scintillation yield at $-35^{\circ} \mathrm{C}$ suggests that energy resolution of $\mathrm{Csl}(\mathrm{Tl})$ should he optimized at this temperature. However, the long decay time is on the order of the longest commercially-available amplifier shaping time, resulting in a s.gnificant ballistic deficit. In fact, the hallistic deficit 
causes the amplifier output pulse amplitude (or photopeak centrold) to decrease with temperature below abou! $10^{\circ} \mathrm{C}$ for all amplifier shaping times, even though the scintillation yield is increasing down to $-35^{\circ} \mathrm{C}$. Thus, improving the energy resolution of $\mathrm{CsI}(\mathrm{TI}) /$ photodiode detectors below room temperature is not as promising as originally hoped. Since the scintillation yield variations with temperature are small over the range of -50 to $+50^{\circ} \mathrm{C}$, other factors, such as decay times and thermoluminescence, will dictate the optimum operating temperature.

The modifled Bollinger-Thomas (MBT) method was used to determine the rise and decay time constants of CsI(TI). The SSW method was used to confirm the primary decay times, $\tau_{1}$ and $\tau_{2}$, determined by the MBT method and to confirm the assumptions regarding temperature dependence that were necessary to use the SSW method for the absolute scintillation yield measurements. There was an apparent systematic bias in the SSW decay times due to inaccurate potentiometer calibration, but for $\mathrm{T} Z-10^{\circ} \mathrm{C}$ the MBT and SSW results show similar temperature dependence. Consequenily, we believe that the MBT results more accurately depict the rise and decay times of CsI(TI) and their temperature dependence. However, we also believe that the application of the SSW method at room temperature accurately models the $\mathrm{CsI}(\mathrm{TI})$ behavior.

The two primary decay time constants determined by the MBT method, $\tau_{1}$ and $\tau_{2}$. were found to increase approximately exponentially with inverse temperature. A significant rise time constant was found to populate $\tau_{1}$ and was also observed to increase exponentially with inverse temperature. The two different decay times can be explained by the presence of two different thallium luminescence centers. An exponential rise heing associated with one of the decays while the other decay has an instantaneous rise suggests that two different types of holes are trapped at the different thallium centers. At room temperature, our hest estimates of $\tau_{\text {rise }} \tau_{1}$, and $\tau_{2}$ are $19.6 \mathrm{~ns}, 679 \mathrm{~ns}$, and $3.34 \mu \mathrm{s}$. For Ts $-30^{\circ} \mathrm{C}$ and $T=-1000^{\circ} \mathrm{C}$, additional decays were observed and the former was found to increase exponentially with inverse temperature. The SSW method was found to reach its limits of 
applicability duc to the length of $\tau_{2}$ at about $-60^{\circ} \mathrm{C}$. The accurate determination of $\tau_{2}$ using the modified Bollinger-Thomas method was complicated by poor signal-to-noise. The signal-to-noise became worse with decreasing temperature due to the increasing decay umes and could be further degraded by thermoluminescence light.

An ultra-fast decay component was confirmed. Our best estimate of the decay time is $<0.5 \mathrm{~ns}$, but it has been previously reported to be $<10 \mathrm{ps}$ by Deich, et al. [DEI89]. The yield of the ultra-fast component appears to be nearly independent of temperature and accounts for roughly $0.2 \%$ of the total light yield for the PMT used (about 100 ) photons/ MeV for gamma rays). This yield is too small to be seen in our emission spectrum measurements, but has been reported to have a broad emission band that peaks around 620 $\mathrm{nm}$ [DEI89]. The ultra-fast component is of limited use in gamma-ray experiments hecause of this long wavelength of emission. The quantum efficiency of typical photocathodes at $620 \mathrm{~nm}$ is very low, such that the yield will be $<1$ photoelectron/MeV. Although the quantum efficiency of photodiodes is typically very good at $620 \mathrm{~nm}$. the photodiode noise will tend to ohscure this small signal. Consequently, using CsI(TI) to produce timing signals from gamma-ray events will result in poor leading edge timing because of the significant rise time. However, it may be possible to use the ultra-fast component to produce good timing signals in high energy physics applications.

Although the SSW method has been demonstrated for use with PDs only, its applicability should extend to PMTs that have a known gain. Likewise, the method could he used for the calibration of any scintillator, but its use is primarily of interest when hallistic deficit renders other methods inaccurate. Scintillators with a single decay time constant will be much easier to model with the SSW method because the use of $k_{1}$ and $k_{2}$ will not he necessary. As mentioned, the SSW method is not an accurate method of determining rise and decay times. However, if the rise and decay times of a scintillator are known at any temperature, the SSW method can be effectively used to calibrate amplifier output pulse height to charge created in the photodetector at that temperature. The SSW 
method is the only method that we know of that accounts for ballistic deficit due to long scintillation decay times and thus accurately callbrates CsI(TI) scintillation pulses.

\subsection{Future Work}

CsI(TI) coupled to a silicon PIN photodiode has become an established detector for gamma ray and charged particle applications. Even though present day commercially. available nuclear electronics do not fully utilize the CsI(TI) scintillation yield, energy resolution as good or slightly better than NaJ(TI) coupled to a PMT can easily be achieved. In the last decade, the development of large area, low noise PDs has been largely motivated by the interest in using such photodetectors with CsI(TI). Further improvements in the manufacturing of PDs will probably result in larger area. lower noise silicon PDs. Several other means of improving the CsI(TI)/PD detector ure heing pursued.

One means of improving the signal-10.noise by decreasing the noise is the development of charge-sensitive preamplifiers that are specifically designed to match PD characteristics. To date, hybrid preamplifiers have heen used extensively with modest performance improvements and a significant improvement in detector/preamplifier size. Another promising solution is to fabricate the PD and preamplifier on the same wafer of silicon. Both types of preamplifiers cut down on the noise due to capacitance and the susceptibility of the detector to microphonies. Unfortunately, decreasing the series noise that is primarily due to capacitance is less important than decreasing the parallel noise that is primarily due to the PD dark current. Due to the long decay time of CsI(TI) it is desirable to use an amplifter shaping time of at least $10 \mu \mathrm{s}$. Since series and parallel noise are inversely and direcily proportional to amplifier shaping time, respectively, it is more important to decrease dark current than capacitance. Nevertheless, designing preamplifiers specifically for PDs is a step in the right direction.

Another possibility for improving detector performance involves the development of new linear amplifiers with shaping times as long as 20 or $30 \mu \mathrm{s}$. If such an amplifier 
were available, then the temperature dependence of CsI(TI) could be used with a very low capacitance PD to improve energy resolution. At room temperature the detector would have poor energy resolution due to the high dark current that will be inherently present in low capacitance PDs, but the CaI(TI)/PD detector could be cooled to $-30^{\circ} \mathrm{C}$ to significantly decrease the dark current. Al $-30^{\circ} \mathrm{C}$, the CsI(TI) scintillation yield is maximized, hut the long decay time is about 9 us. A 20 to $30 \mu \mathrm{s}$ amplifier shaping time would preserve ahout 94\% to 98\% of the full pulse amplitude, respectively, and have a combination of parallel and series noise that is minimized. Unfortunately, an amplifier shaping time of 20 to $30 \mu \mathrm{s}$ would disqualify this system for many applications due to pulse pile-up. The use of a gated integrator, instead of the semi-Oaussian shaping that is typically utilized by commercially-avallable amplifiers, could reduce the pulse pile-up problems. A gated integrator could similarly preserve about $94 \%$ to $98 \%$ of the CsI(T) pulse amplitude for 20 to $30 \mu \mathrm{s}$ integration. respectively, but the total length of a semi-Gaussian shaped pulse is typically about four times as long as a gated integrator pulsc.

Two other promising lechnologies are the fabrication of avalanche PDs [JAM92] and silicon drif PDs (AVS90). One reason that PDs have a difficult time competing with PMTs is that PDs have unity gain and consequently small signals. To date, avalanche PDs, with a typical gain of IOX), have demonstrated similar energy resolution to standard PDs with CsI(TI) at room temperature. The improvements in gain are offset by non-uniformity across the sensitive area of the PD and hy increases in hoth series and parallel noise. However, the fabrication of avalanche PDs is a reasonably new technology that may see dramatic improvements in the near future. Silicon drif PDs are another new technology that could significanily enhance the use of $\mathrm{Cs}(\mathrm{T})$ with a PD. By using electric field gradients to localize the charge created by the scintillation photons, silicon drift PDs can greatly increase the sensitive area of a PD without increasing noise. To date, these PDs are primarily used for position sensitive applications. However, one can easily envision the coupling of avalanche and silicon drift technologies to produce a large photodetector with 
appreciable gain and low noise. This PD would utilize the silicon drift technology to direct all of the electrons to a smull avalanche region, and thus alleviate the problems of nonuniformity and high noise that have plagued avalanche PDs.

In addition to these new types of PDs, the use of wide band gap semiconductors as PDs is also being developed. Independent of which means are pursued toward improving signal-10-noise for the CsI(TI)/PD, it is always important to consider the scintillation characteristics of CsI(TI). Due to the long decay time, CsI(TI) is a more difficult scintillator In fully utilize than most. Consequently, the characterization that we have performed should aide in the further development of PDs, preamplifiers, and amplifiers for use with CsI(TI). 
APPENDICES 


\section{APPENDIX A \\ UNCERTAINTY OF CENTROID AND FULL-WIDTH AT HALF MAXIMUM DETERMINATIONS}

The accurate determination of the centroid $\left(x_{0}\right)$ and full-width at half maximum (FWHM) is crucial to applications ranging from detector characterization to resolving multiplet peaks in gamma-ray spectroscopy. The uncertainties associated with determining the centroid and FWHM are fundamental to the knowledge of accuracy. Furthermore, assuming that histogrammed data can be represented by the continuous Gaussian shape will have some applicability limits that will affect the accuracy of determining the centroid and FWHM. This binning effect should be minimized for optimum accuracy [CIA70, HEA84, HUT73].

In gamma-ray spectroscopy it is often assumed that photopeaks have the Gaussian shape and thus FWHM=2.355 $\sigma$, where $\sigma$ is the standard deviation of the photopeak distribution. Conventional graphical methods measure the FWHM exactly to within the statistical limits of the data and thus will not be severely hampered by this assumption in most cases, but may result in a relationship between FWHM and $\sigma$ that is different from FWHM $=2.355 \sigma$. On the other hand, any method that utilizes fitting a Gaussian to the data will suffer if the distribution is not truly Gaussian. A reasonable visual fit will not necessarily yield an accurate FWHM calculation since FWHM=2.3550 is based on the assumption of a Gaussian shape. Calculating the central moments will have a similar problem for data that does not have a Gaussian shape, but has the benefit of being able to assess whether the data has a Gaussian shape.

To assess the uncertainty in centroid and FWHM determinations we have used a Monte Carlo program to generate Gaussian shaped peaks for analysis. The peaks were 
analyzed by both non-linear least squares fitting and the calculation of moments. In addition, the expected uncertainty in the moments calculations have been derived.

\section{A.1 Error Propagation}

\section{A.1.1 Definitions of Moment Equations}

For a distribution $y(x)$, the $\mathrm{i}^{\text {th }}$ moment, $\mathrm{m}_{\mathrm{i}}$, and $\mathrm{i}^{\text {th }}$ central moment, $\mu_{\mathrm{i}}$, are defined by $m_{\mathrm{i}}=\mathrm{E}\left[\mathrm{x}_{\mathrm{i}}\right]$ and $\mu_{\mathrm{i}}=\mathrm{E}\left[\left(\mathrm{x}-\mathrm{x}_{0}\right)^{\mathrm{i}}\right]$, respectively, where $\mathrm{E}[]$ is the expected value of the bracketed term and $\mathrm{x}_{0}$ is the centroid of the distribution or the first moment, $\mathrm{x}_{0}=\mathrm{m}_{1}=\mathrm{E}[\mathrm{x}]$. The second central moment of $y(x)$ is the variance of the distribution, $\mu_{2}=\sigma^{2}$, while the third and fourth central moments of $y(x)$ determine the coefficients of momental skewness, sk, and kurtosis, $\mathrm{k}$, which are defined by

$$
\mathrm{sk}=\frac{\mu_{3}}{\mu_{2}^{3 / 2}},
$$

and

$$
k=\frac{\mu_{4}}{\mu_{2}^{2}}-3 .
$$

The central moments can be broken down to sums and products of expected values of powers of $\mathrm{x}$ and the centroid:

$$
\begin{aligned}
& \mu_{2}=\mathrm{E}\left[\left(\mathrm{x}-\mathrm{x}_{0}\right)^{2}\right]=\mathrm{E}\left[\mathrm{x}^{2}\right]-\mathrm{x}_{0}^{2}, \\
& \mu_{3}=\mathrm{E}\left[\left(\mathrm{x}-\mathrm{x}_{0}\right)^{3}\right]=\mathrm{E}\left[\mathrm{x}^{3}\right]-3 \mathrm{E}\left[\mathrm{x}^{2}\right] \mathrm{x}_{0}+2 \mathrm{x}_{0}^{3}, \\
& \mu_{4}=\mathrm{E}\left[\left(\mathrm{x}-\mathrm{x}_{0}\right)^{4}\right]=\mathrm{E}\left[\mathrm{x}^{4}\right]-4 \mathrm{E}\left[\mathrm{x}^{3}\right] \mathrm{x}_{0}+6 \mathrm{E}\left[\mathrm{x}^{2}\right] \mathrm{x}_{0}^{2}-3 \mathrm{x}_{0}^{4},
\end{aligned}
$$

such that the moments, $m_{i}$, are combined to determine the central moments, $\mu_{i}$ [STU87].

For a Gaussian distribution, we know that $\mathrm{FWHM}=2.355 \sigma$ and $\mathrm{sk}=\mathrm{k}=0$ [STU87]. In practice, given a discrete distribution $y(x)$ we can calculate the centroid, variance, skewness, and kurtosis using the definition of the expected values of the powers of $\mathrm{x}$ for discrete data: 


$$
E\left[x^{n}\right]=\frac{\sum_{i=1}^{I} x_{i}^{n} y_{i}}{\sum_{i=1}^{1} y_{i}},
$$

where $x_{i}$ is the $i^{\text {th }}$ bin and $y_{i}=y\left(x_{i}\right)$ is the number of counts in the $i^{\text {th }}$ bin, respectively, of a distribution with I bins [STU87]. Using Eqn. (A.6) to calculate $m_{1}$ and in Eqns. (A.3A.5), the centroid, variance, skewness, and kurtosis of any distribution can be determined, but result in $\mathrm{sk}=0$ and $\mathrm{k} \approx 0$ when the distribution is of Gaussian origin.

\section{A.1.2 Statistical Uncertainty in Moments Calculations}

To determine the uncertainty of the moments calculations used to calculate $x_{0}$ and FWHM, the statistical uncertainty of the $y_{i}$ 's were propagated through the moments calculations. The error propagation formula for a function $F(x, y, z)$ where $x, y$, and $z$ are uncorrelated random variables is

$$
\operatorname{var}(F)=\left(\frac{\partial F}{\partial x}\right)^{2} \operatorname{var}(x)+\left(\frac{\partial F}{\partial y}\right)^{2} \operatorname{var}(y)+\left(\frac{\partial F}{\partial z}\right)^{2} \operatorname{var}(z),
$$

where $\operatorname{var}(F), \operatorname{var}(x), \operatorname{var}(y)$, and $\operatorname{var}(z)$ are the variance associated with $F, x, y$, and $z$, respectively. Since $E\left[x^{n}\right]$ has $y_{i}^{\prime}$ s in both the numerator and denominator, the partial derivatives must involve the quotient rule. It can not be assumed that the peak area, $A=\Sigma y_{i}$, is uncorrelated with the numerator of any expected value calculation. The error calculation for the distribution variance has correlated $E\left[x^{\mathrm{n}}\right]^{\prime} s$ (i.e. $E[x]$ is correlated with $\left.E\left[x^{2}\right]\right)$ because of the $y_{i}$ 's in each of the $E\left[x^{n}\right]$ 's. Thus to apply Eqn. (A.7) to the moments calculations, the $y_{i}^{\prime}$ s are assumed to be the uncorrelated random variables.

Using this assumption and the error propagation formula, the variance of the centroid of a distribution is

$$
\operatorname{var}\left(x_{0}\right)=\sum_{j=1}^{1}\left(\frac{\partial x_{0}}{\partial y_{j}}\right)^{2} \operatorname{var}\left(y_{j}\right) .
$$


The variance of the bin contents, $\operatorname{var}\left(y_{j}\right)$, is governed by Poisson statistics and is thus $\operatorname{var}\left(y_{j}\right)=y_{j}$. The partial derivative for the $j^{\text {th }}$ bin is

$$
\frac{\partial x_{0}}{\partial y_{j}}=\frac{\left(\sum_{i=1}^{1} y_{i}\right)\left(x_{j}\right)-\left(\sum_{i=1}^{1} x_{i} y_{i}\right)}{\left(\sum_{i=1}^{1} y_{i}\right)^{2}}=\frac{1}{\sum_{i=1}^{1} y_{i}}\left[x_{j}-x_{0}\right]
$$

Substituting Eqn. (A.9) into Eqn. (A.8) yields

$$
\begin{aligned}
\operatorname{var}\left(x_{0}\right) & =\frac{1}{\left(\sum_{i=1}^{1} y_{i}\right)^{2}} \sum_{j=1}^{1}\left[x_{j}-x_{0}\right]^{2} y_{j} \\
& =\frac{1}{\sum_{j=1}^{1} y_{i}}\left[\frac{\sum_{j=1}^{1} x_{j}^{2} y_{j}}{\sum_{j=1}^{1} y_{j}}-2 x_{0} \frac{\sum_{j=1}^{1} x_{j} y_{j}}{\sum_{j=1}^{1} y_{j}}+x_{0}^{2} \frac{\sum_{j=1}^{1} y_{j}}{\sum_{j=1}^{1} y_{j}}\right] \\
& =\frac{1}{A}\left[E\left(x^{2}\right)-2 x_{0}^{2}+x_{0}^{2}\right] \\
& =\frac{1}{A}\left[E\left(x^{2}\right)-x_{0}^{2}\right] \\
\operatorname{var}\left(x_{0}\right) & =\frac{\sigma^{2}}{A},
\end{aligned}
$$

where $\sigma$ is the standard deviation of the original distribution and is given by

$$
\sigma=\sqrt{\sigma^{2}}=\sqrt{E\left(x^{2}\right)-[E(x)]^{2}}=\sqrt{\frac{\sum_{i=1}^{1} x_{i}^{2} y_{i}}{\sum_{i=1}^{1} y_{i}}-\left[\frac{\sum_{i=1}^{1} x_{i} y_{i}}{\sum_{i=1}^{1} y_{i}}\right]^{2}} .
$$

Using Eqns. (A.7) and (A.11), and the assumption of uncorrelated $y_{i}^{\prime}$, the variance of the standard deviation of the distribution can be determined by

$$
\operatorname{var}(\sigma)=\sum_{j=1}^{1}\left(\frac{\partial \sigma}{\partial y_{j}}\right)^{2} \operatorname{var}\left(y_{j}\right) .
$$

Again $\operatorname{var}\left(y_{j}\right)=y_{j}$, and the partial derivative with respect to the $j^{\text {th }}$ bin contents is determined from Eqn. (A.11) to he 


$$
\frac{\partial \sigma}{\partial y_{j}}=\frac{1}{2 \sigma \sum_{i=1}^{1} y_{i}}\left[x_{j}^{2}-2 x_{0} x_{j}+2 x_{0}^{2}-E\left(x^{2}\right)\right] .
$$

The variance is then

$$
\begin{aligned}
\operatorname{var}(\sigma)= & \frac{1}{\left(2 \sigma \sum_{i=1}^{1} y_{i}\right)^{2}} \sum_{j=1}^{1}\left[x_{j}^{2}-2 x_{0} x_{j}+2 x_{0}^{2}-E\left(x^{2}\right)\right]^{2} y_{j} \\
= & \frac{1}{4 \sigma^{2}\left(\sum_{i=1}^{1} y_{i}\right)^{2}}\left[x_{j}^{4}-4 x_{0} x_{j}^{3}+8 x_{0}^{2} x_{j}^{2}-2 E\left(x^{2}\right) x_{j}^{2}-8 x_{0}^{3} x_{j}\right. \\
& \left.+4 x_{0} E\left(x^{2}\right) x_{j}+4 x_{0}^{4}-4 x_{0}^{2} E\left(x^{2}\right)+\left[E\left(x^{2}\right)\right]^{2}\right] y_{j} \\
= & \frac{1}{4 \sigma^{2} \sum_{i=1}^{1} y_{i}}\left[\frac{\sum x_{j}^{4} y_{j}}{\sum y_{j}}-4 x_{0} \frac{\sum x_{j}^{3} y_{j}}{\sum y_{j}}+8 x_{0}^{2} \frac{\sum x_{j}^{2} y_{j}}{\sum y_{j}}-2 E\left(x^{2}\right) \frac{\sum x_{j}^{2} y_{j}}{\sum y_{j}}\right.
\end{aligned}
$$

regrouping the terms and recalling the definition of $\mu_{4}$

$$
\operatorname{var}(\sigma)=\frac{1}{4 \sigma^{2} \mathrm{~A}}\left[\mu_{4}-\left(\sigma^{2}\right)^{2}\right]
$$

where $\sigma^{2}=\mu_{2}$ is the distribution variance.

When the underlying distribution has a Gaussian shape, the variance of the standard deviation, $\operatorname{var}(\sigma)$, further simplifies because of the definition of the coefficient of kurtosis, Eqn. (A.2), (if Gaussian, then $k=0$, then $\mu_{4}=3 \mu_{2}^{2}=3\left(\sigma^{2}\right)^{2}$ ), consequently

$$
\operatorname{var}(\sigma)=\frac{1}{4 \sigma^{2} \mathrm{~A}}\left[3\left(\sigma^{2}\right)^{2}-\left(\sigma^{2}\right)^{2}\right]=\frac{\sigma^{2}}{2 \mathrm{~A}} \text {. }
$$


Recalling that FWHM $=2.355 \sigma$ and that the standard deviation of a parameter $q, s_{q}$, is related to the variance of $q$ by $\operatorname{var}(q)=s_{q}^{2}$, we see that the theoretical relative standard deviation of the FWHM is

$$
\frac{s_{\mathrm{FWHM}}}{\mathrm{FWHM}}=\frac{\sqrt{\operatorname{var}(\mathrm{FWHM})}}{\mathrm{FWHM}}=\frac{\sqrt{2.355^{2} \operatorname{var}(\sigma)}}{2.355 \sigma}=\frac{\sqrt{2.355^{2} \sigma^{2} / 2 \mathrm{~A}}}{2.355 \sigma}=\frac{1}{\sqrt{2 \mathrm{~A}}}
$$

and is thus independent of peak width.

\section{A.2 Peak Generation Program}

To test the results of the error analysis a computer program was developed. The program produces Gaussian shaped distributions comparable to a background subtracted photopeak [CIA68, FAL84]. This method of peak generation was chosen over actual experimental data for its diversity in creating peaks of different shapes and the ease of creating thousands of peaks.

The program creates a peak with $8 \sigma(\approx 3.4 \mathrm{FWHM})$ total width, given a peak area and FWHM. To approximate an experimental peak the centroid bin was offset randomly, chosen from -0.5 to +0.5 . A peak that is symmetric about a single bin has 0.0 offset, whereas a peak that is symmetric about two bins has either -0.5 or +0.5 offset, as shown in Figure A.l. After choosing the offset, a Gaussian distribution with prescribed peak area and FWHM was integrated into a discrete distribution (bins) such that the chosen offset was satisfied. Subsequently, Poisson noise was applied to the content of each bin and thus a realistic model of a peak was achieved (see Figure A.2).

The prescribed centroid and FWHM were compared with the moments calculations or least squares fitting results for many different peaks with the same underlying distribution to determine an experimental standard deviation. The expected standard deviation of the centroid and FWHM from the moments calculations can be determined from Eqns. (A.10) and (A.16), respectively. Thus, comparing the experimental and expected standard deviations of the moments calculations we can assess the effect of 

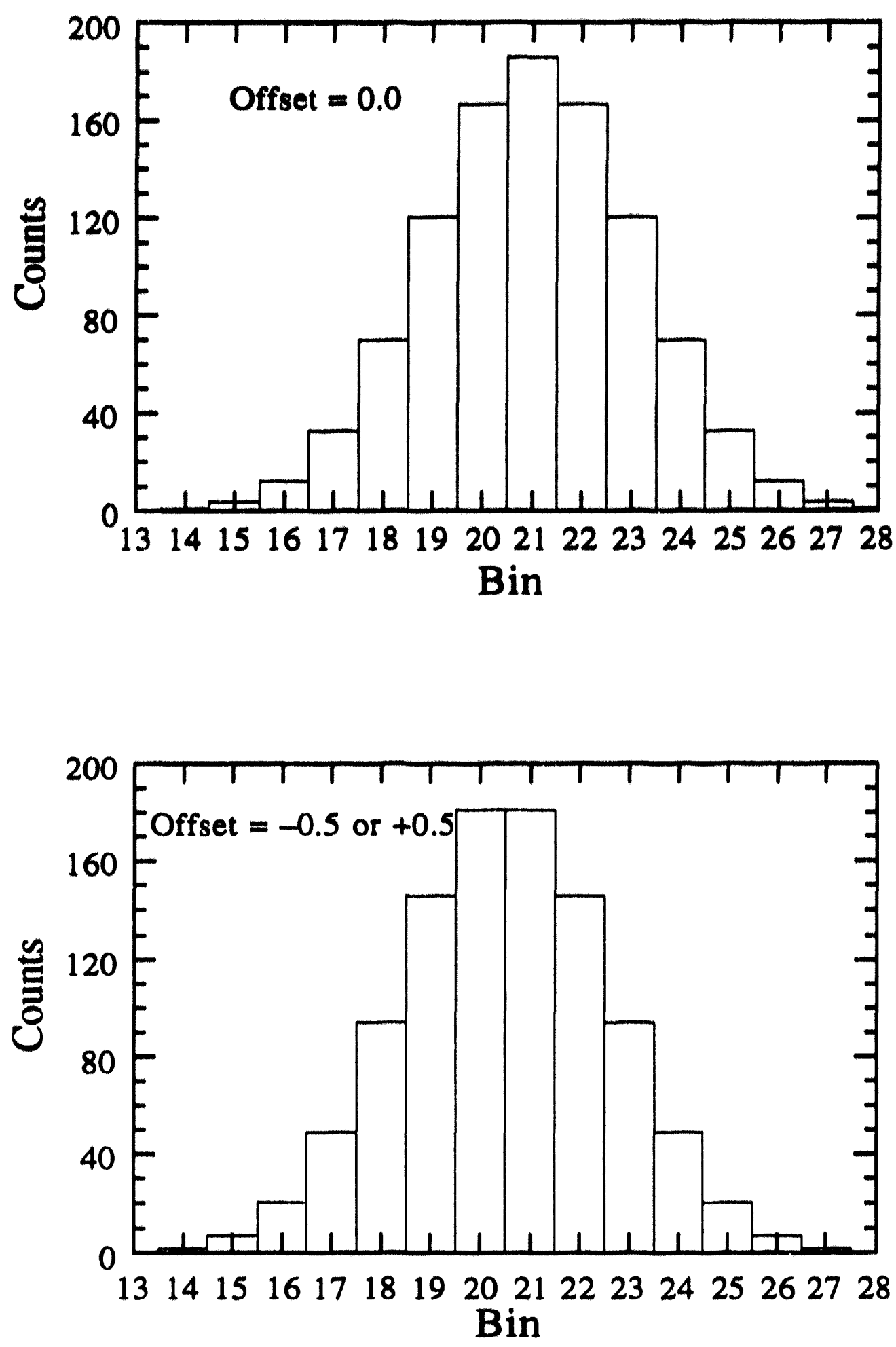

Figure A.1: Examples of centroid offset. 


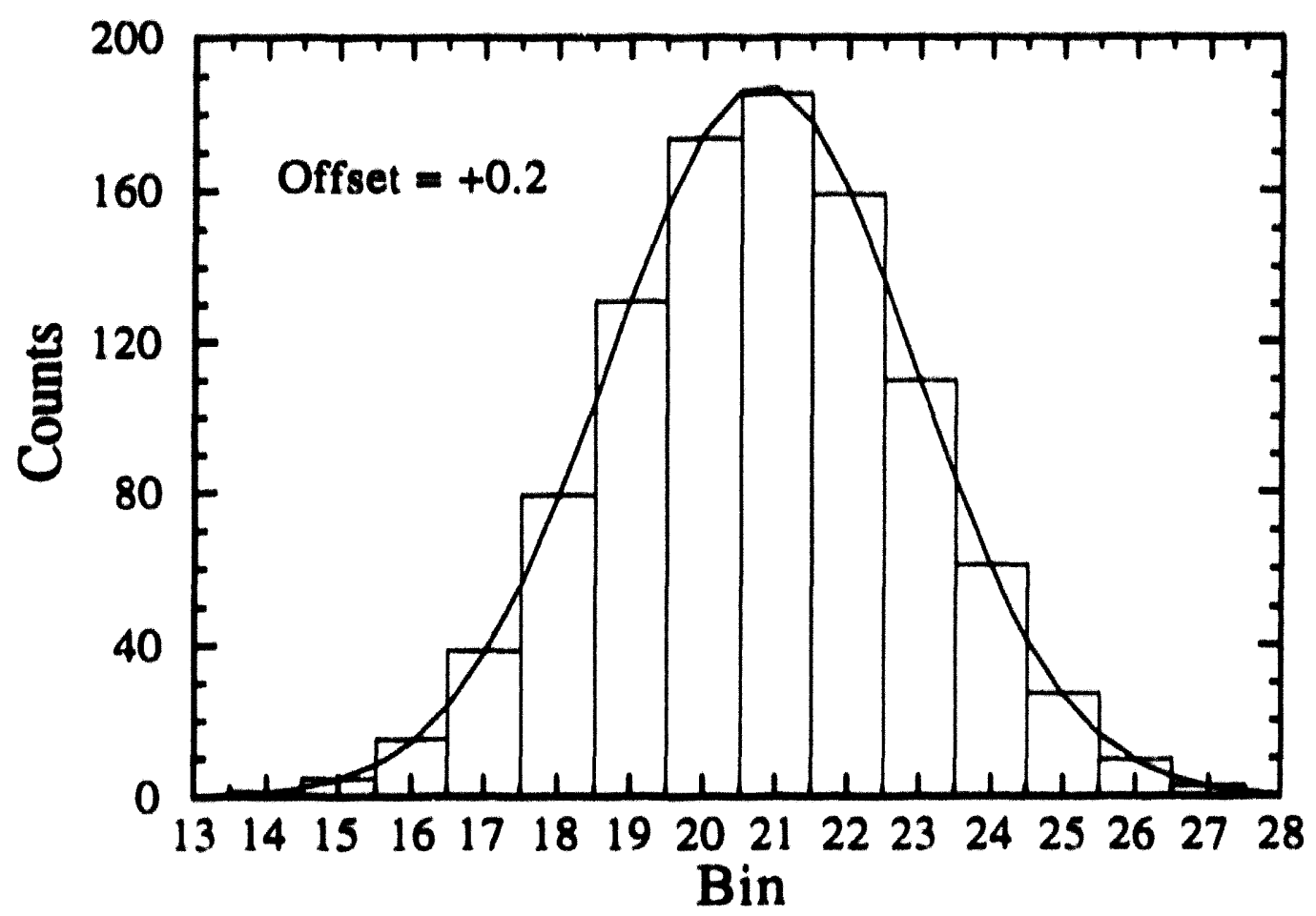

(a)

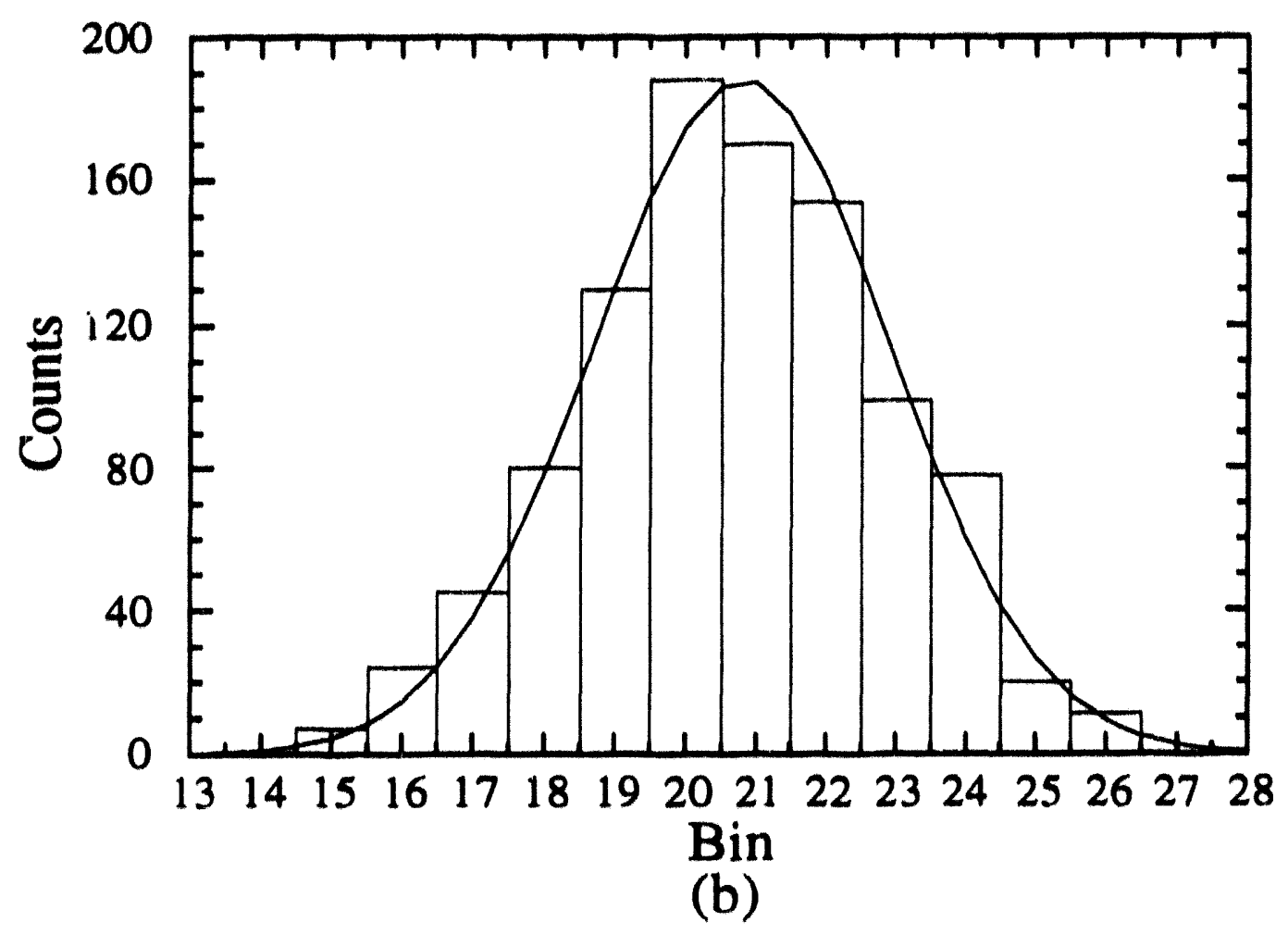

Figure A.2: Examples of (a) peak integration and (b) addition of Poisson noise. 


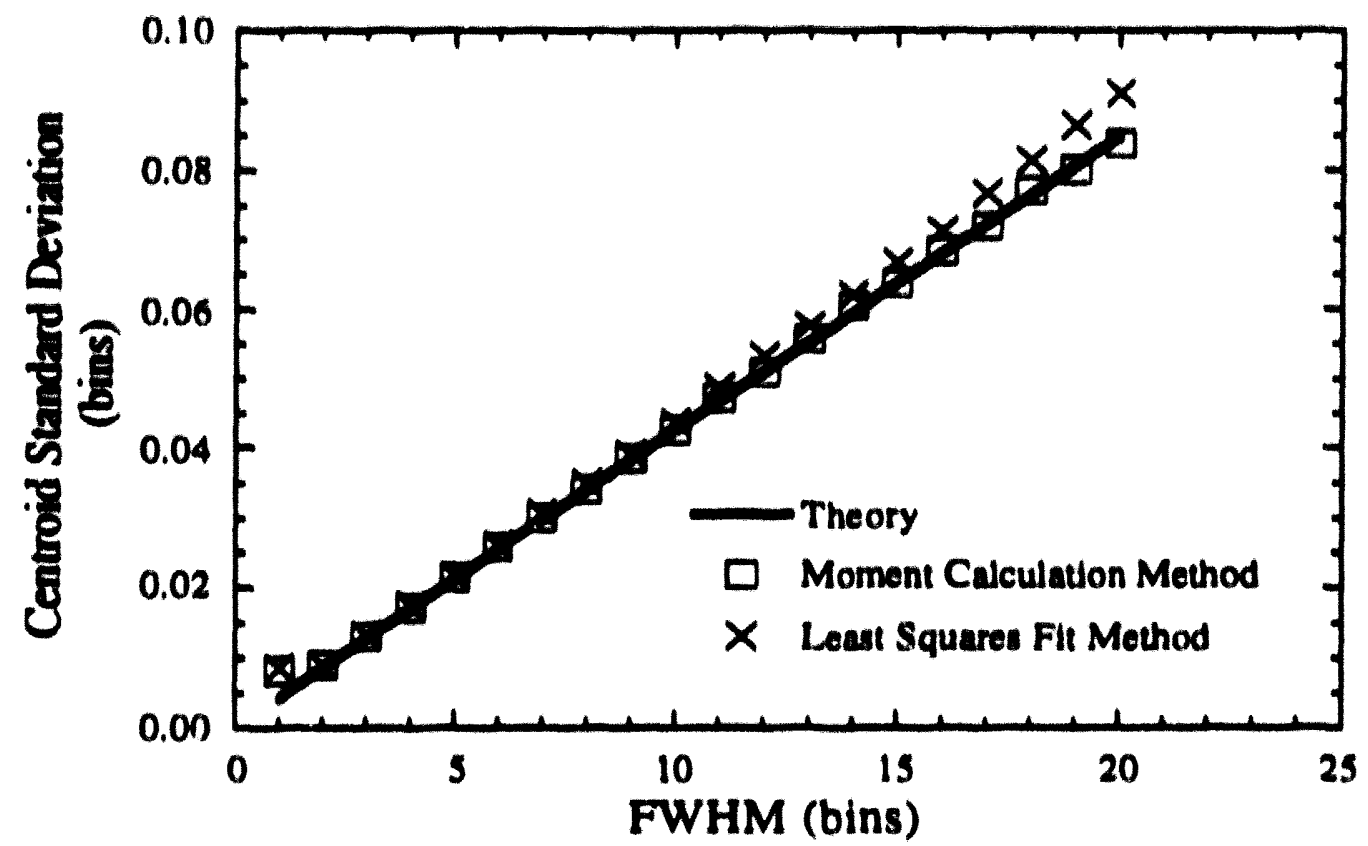

Figure A.3: Centroid results of moments calculations and least squares fitting for a peak area of 10,000 counts.

binning data, i.e. for comparable expected and experimental standard deviations we conclude that binning has negligible effect and that the discrete data can be approximated by a Gaussian distribution.

\section{A.3 Results}

The centroid and FWHM of peaks generated by the program were determined by moments calculation and by a non-linear least squares fitting program. The results are shown in Figures A.3 and A.4 along with the theoretical values derived in Section A.1 for a peak area of 10,000 counts. Each point in the figures represents the experimental standard deviation derived from 10,000 different peaks generated by the program. From Figure A.4 we see that the theoretical and moments calculation method results diverge with decreasing FWHM. For FWHM 211 bins, the results of the moments calculation method are within $5 \%$ of the theoretical values. We have concluded that this divergence is due to the binning 


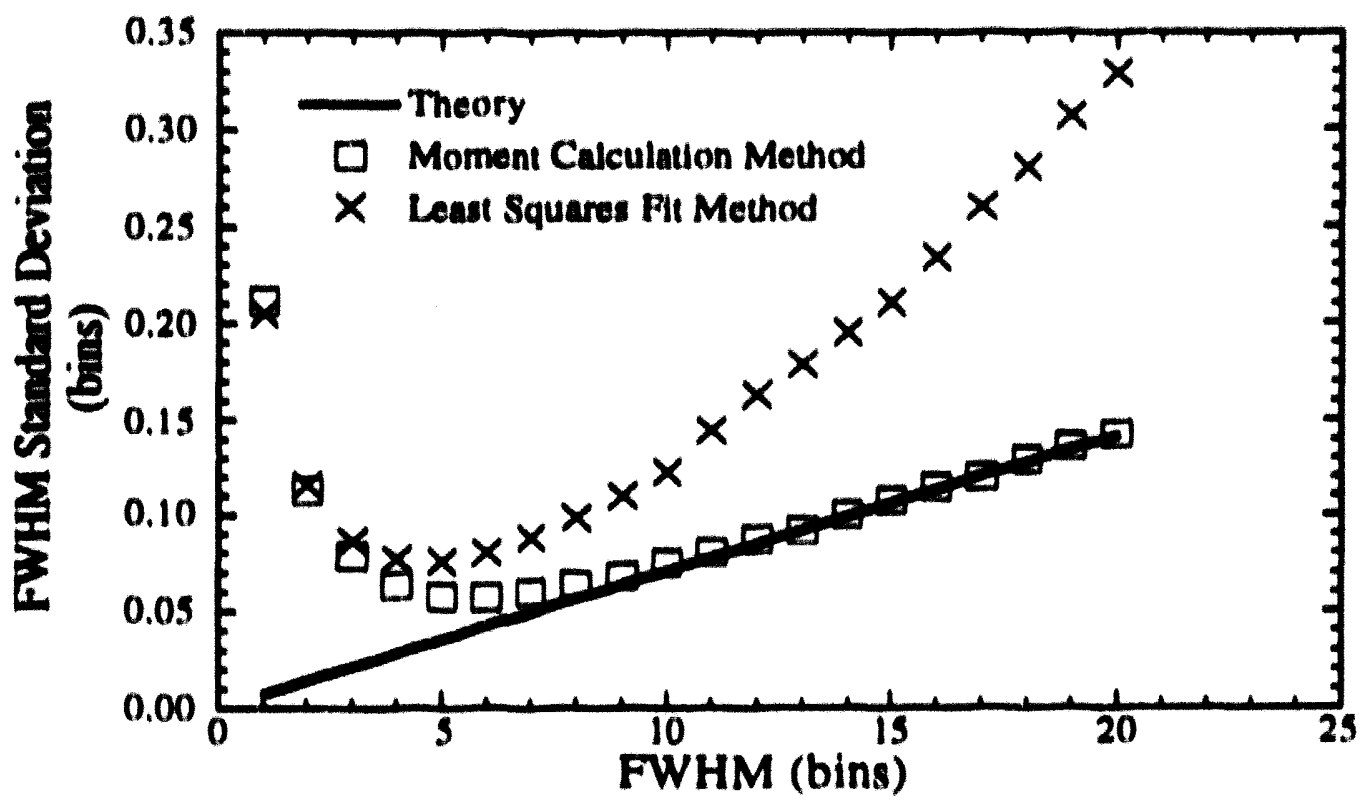

(a)

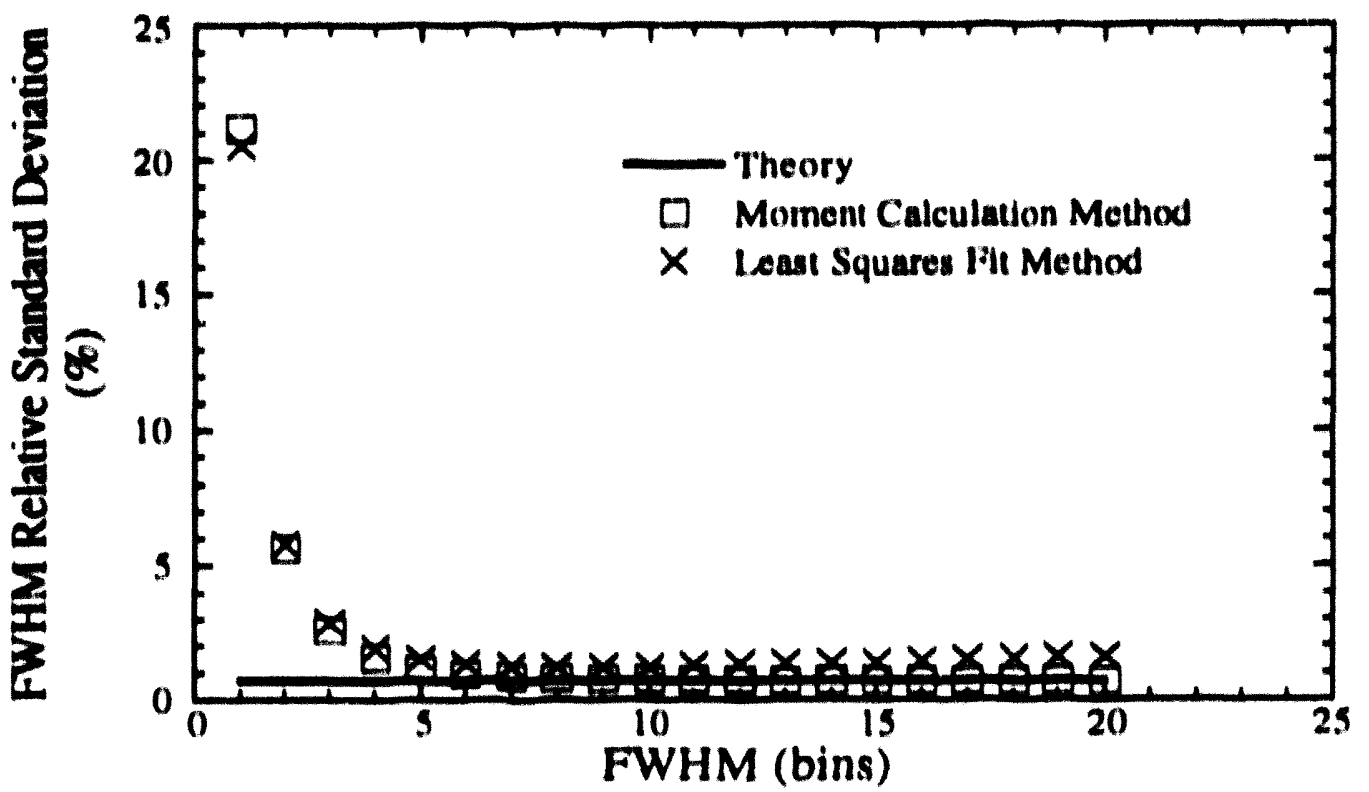

(b)

Figure A.4: FWHM results of moments calculations and least squares fitting for a peak area of 10,000$)$ counts. 


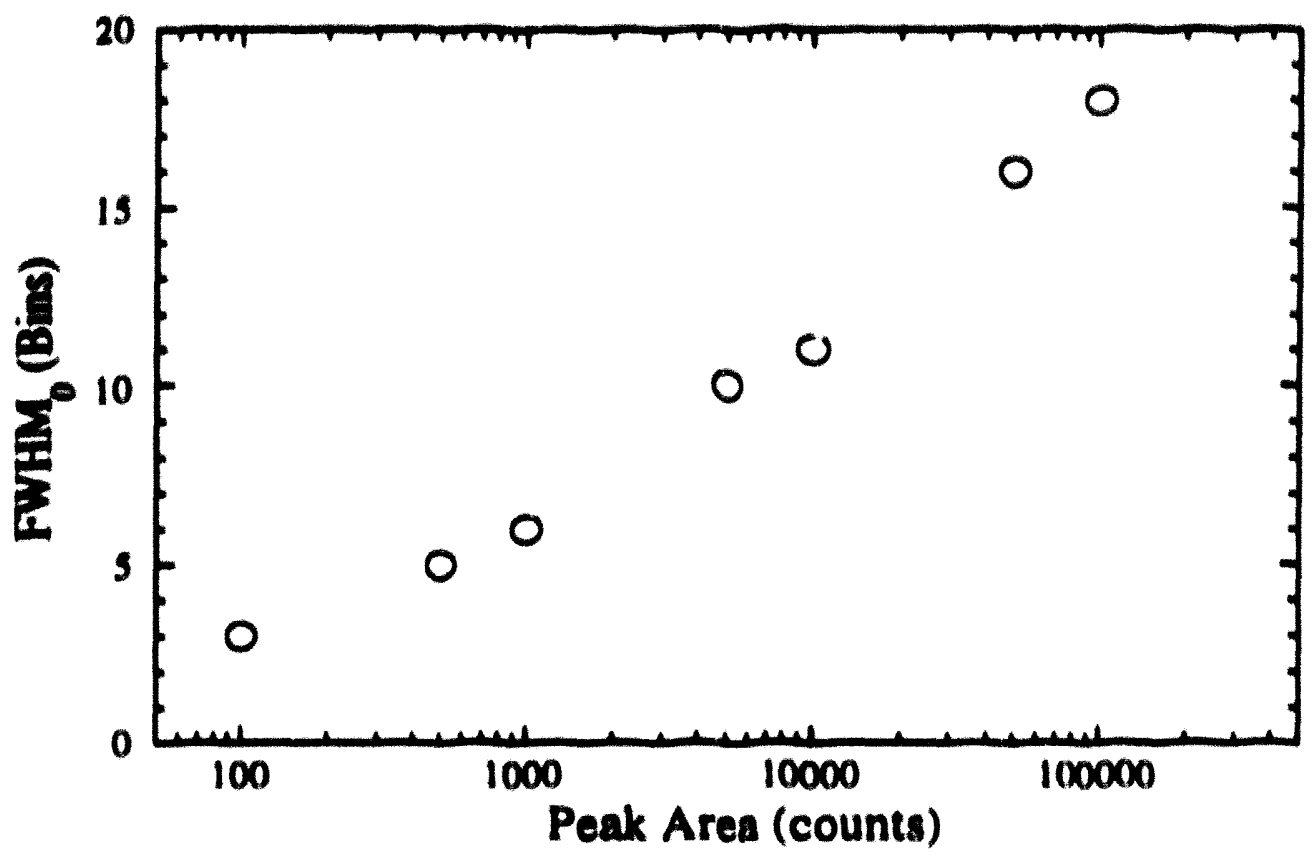

Figure A.5: FWHM above which moments calculation uncernainties are within 5\% of the theoretical predictions.

effect. The value of FWHM helow which the simulated and theoretical results diverge by 25\%, FWHM 0 , is dependent on the peak area, as shown in Figure A.S. It is also observed that the least squares fituing method has a minimum uncertainty at ahout FWHM=10 bins for peak areas of 10,000 counts. This minimum is also dependent on peak area.

Above FWHM $M_{0}$ the uncertainty in determining the FWHM will be minimized. Unfortunately, the standard deviation of the centroid, $s_{x_{0}}$ is not minimized under the same conditions, as seen in Figure A.3. We notice from the figure that the binning effect does not affect $s_{x_{0}}$ until FWHM $<2$, this result is independent of peak area unlike the previously described FWHM . However, it is observed that $s_{x_{0}}<0.1$ hins for FWHMS20) and $x_{0}$ is typically large (hundreds or thousands of bins), the combination of which yields a small relative uncertainty. 


\section{A.4 Conclualons}

The uncernainty in determining the centroid of a Gaussian shaped peak is minimized hy using either the least squares fitting or first moment calculation method and hy limiting the width of the FWHM to ahout 2 bins. Similarly, the moments calculation method minimizes the uncertainty in determining the FWHM of a Gaussian shaped peak and approaches the statistical limit with increasing FWHM. While the least squares filting method results in a larger uncertuinty in the FWHM than the moments calculation method. the uncertainty is minimized at a specific value of FWHM that is dependent on peak area. Peak searching and multiplet resolving routines are examples of applications that will require consideration of uncertainty minimization. 


\section{APPENDIX B \\ DETECT (LIGHT COLLECTION EFFICIENCY) INPUT AND OUTPUT FILES}

The visible light transport program DETECT that was used to determine the light collection efficiency of our CsI(TI)/photodiode system was originally written by Thomas F. Knoll [KNO88], then of The University of Michigan. As described in Chapter IV, we have modified the program to account for the Fresnel reflections of the photons at the front of the photodiode. With this modification, the program can be used to determine light collection efficiency, as we have defined it, hy modeling the optical hehavior of a scintillator and photodiode. The program requires an input file that contains the optical and peometric properties of each medium that scintillation photons might travel through on the way to the photodiode. The input file also specifies where and how many scintillation photons are generated. The program then generates photons that travel in random initial directions and follows the fate of each photon until it is either bulk absorhed, surface ahsorhed, lost from the system, timed out (photon trapped in the scintillator), or transmitted into the photodiode. All photons that were transmitted into the photodiode were assumed II subsequently he absorhed in the photodiode. The input file used for our CsI(TI)/photodiode system is listed helow and Fig. B.I shows the resulting geometry that is modeled. The comments in parenthesis have heen added for descriptive purposes. The units for the dimensions in the input file are all in tenths of millimeters. Component $I$ is the CsI(TI) crystal, 2 is the coupling compound, 3 is the resin coating, and 4 is the $\mathrm{SiO}_{2}$. Note that photons could leave the system through the sides of the coupling compound and hy exiting the PD resin coating on the outside millimeter all the way around the PD. However we have modeled these escape paths as vacuums with hlack reflectors at their outside houndaries (components 5, 6, 7, and 8). Thus photons that leave via these paths will he 
recorded as escaped in the output file, but will be included in the surface absorbed count along with photons that are absorbed by the white PTFE tape that is wrapped around the CsI(TI) crystal.

\section{DETECT input file listing}

mult.out

(output nle name)

:wrapped $8 \times 8 \times 8$ cube of $\mathrm{CsI}(\mathrm{TI})$ coupled to PD

DMATI,1.8N

DMAT2,1.5N

DMAT3,1.46N

DMAT4.1.0N

(material 2, coupling compound and PD resin coating with index of refraction of 1 5)

(description of detector)

(material 1, Cal(TI) with index of refraction of 1.8)

(material 3, sillicon dioxide with index of refraction of 1.46)

(material 4, als or vacuum with index of refraction of 1.0 )

DFINI,PAINT,0.98RC

(surface Anish 1, white PTFE tape with reflectance of 0.98 )

DFIN2,DETECT

(surface finish 2, sillicon PDN pholodiode)

DFIN3,POLISH

DFINA,PAINT,O.ORC

(surface finish 3, polished surface at al inierfaces other than finishea 1, 2, and 4) (surface finish 4, black hody absoxher)

DPLNI.0Z

DPLN2,12.

DPLN3.11Z

I)PI.N4,917

DPLN5,40X

DPL.N6.40X

I)PI.N7,40Y

DPLN8,40Y

DPLN9,8Z

DPLN10.101Z

DPLN11.50X

DPLN12,-50X

DPLN13,50Y

IDPLN14,.50Y

COMPI.MATI

SURF, FIN 3,PLN3,ZL,C2

SURF,FINI,PLNS,XS

SURF,FINI,PLN6,XL

SURF,FINI,PLN7,YS

SURF,FINI,PLN8,YI.

SURF,FINI,PLN4,ZS

COMP2,MAT2

SURF, FIN 3,PLN $3, Z S, C 1$

SURF,FIN3,PLNS,XS.CS

SURF,FIN3,PLN6,XL,C6

SURF,FIN3,PLN7,YS,C7

SURF,FIN3,PLN8,YL,C8

SURF,FIN3,PI.N9,ZL.C3 (different planes used to model the detector geometry)

(component 1, $8 \times 8 \times 8 \mathrm{~mm}$ cubic CsI(TI) crystal wrapped with white PTFE tape on five sides and connected on the bottom to component 2. bottom is polished)

(component $2,0.3 \times 8 \times 8 \mathrm{~mm}$ of coupling compound hetween component 1 on top and 3 on the boltom, also in contact on the sides with components 5, 6, 7, and 8, all sides polished) 
COMP3,MAT2

SURF,FIN3,PLN9,ZS,C2,C5,C6,C7,C8

SURF,FIN1,PLN1 1,XS

SURF,FIN1,PLN12,XL

SURF,FIN1,PLN13,YS

SURF,FIN1,PLN14,YL

SURF,FIN3,PLN2,ZL,C4

COMP4,MAT3

SURF,FIN3,PLN2,ZS,C3

SURF,FIN1,PLN11,XS

SURF,FIN1,PLN12,XL

SURF,FIN1,PLN13,YS

SURF,FIN1 PLN14,YL

SURF,FIN2,PLN1,ZL

COMP5,MAT4

SURF,FIN3,PLN9,ZL,C3

SURF,FIN4,PLN11,XS

SURF,FIN3,PLN5,XI,C2

SURF,FIN3,PLN7,YS,C7

SURF,FIN3,PLN8, YL,C8

SURF,FIN4,PLN3,ZS

COMP6,MAT4

SURF,FIN3,PLN9,ZL,C3

SURF,FIN3,PLN6,XS,C2

SURF,FIN4,PLN12,XL

SURF,FIN3,PLN7,YS,C7

SURF,FIN3,PLN8, YL,C8

SURF,FIN4,PLN3,ZS

COMP7,MAT4

SURF,FIN3,PLN9,ZL,C3

SURF,FIN4,PLN11,XS

SURF,FIN4,PLN12,XL

SURF,FIN4,PLN13,YS

SURF,FIN3,PLN7, YL,C2,C5,C6

SURF,FIN4,PLN3,ZS

COMP8,MAT4

SURF,FIN3,PLN9,ZL,C3

SURF,FIN4,PLN11,XS

SURF,FIN4,PLN12,XL

SURF,FIN3,PLN8,YS,C2,C5,C6

SURF,FIN4,PLN14,YL

SURF,FIN4,PLN3,ZS (component $3,0.7 \times 10 \times 10 \mathrm{~mm}$ resin coating between components $2,5,6,7$, and 8 on top and component 4 on the bottom, top and bottom are polished, sides are reflective white ceramic material of $\mathrm{PD}$ packaging)

(component 4, $0.1 \times 10 \times 10 \mathrm{~mm}$ silicon dioxide between component 3 on top and the PD on bottom, top is polished, sides are reflective white ceramic material of $\mathrm{PD}$ packaging)

(components 5, 6, 7, and 8, air or vacuum outside photon escape paths, outside surfaces black, surfaces connecting with components 2,3 , and other air components polished)

GEN,MAT1,11ZS,91ZL,-40XS,40XL,-40YS,40YL (randomly generate photons in CsI(Tl) crystal) LIFE 10000 (maximum lifetime of photons, after which it is timed out or considered trapped) HIST,STEP200,50STEPS SEED29 (format for time of arrival, not decay times but time-of-flight) RUN1000000,DETIMG0,-50XS,50XI,-50YS,50YL,64SQ (initial random number generator seed) END (generate $1,000,000$ photons) 

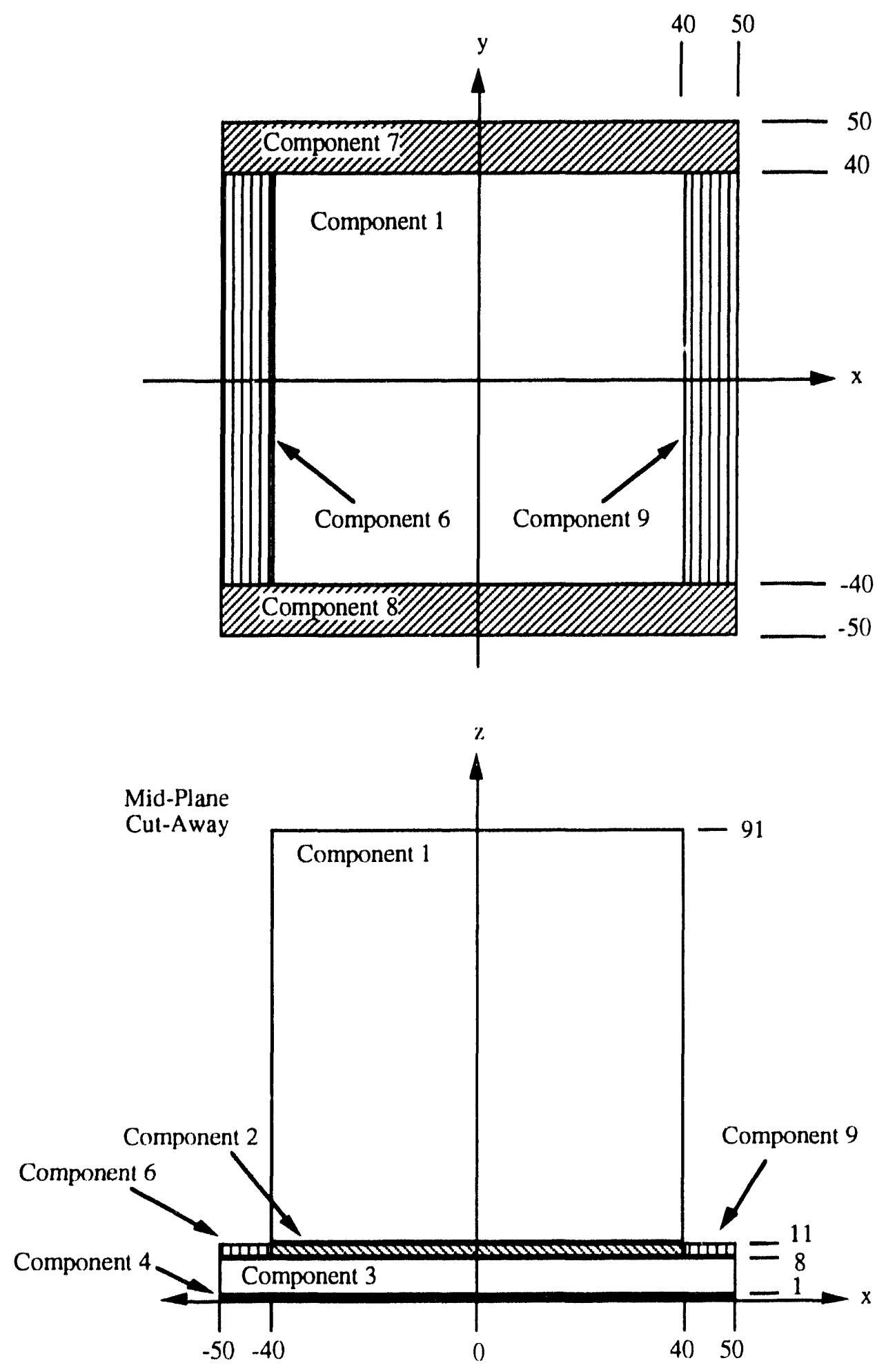

Figure B.1: CsI(T1)/photodiode geometry for DETECT. 
The portion of the DETECT output file that reports the fate of the photons simulated is shown below. As mentioned in Chapter IV, we assumed that bulk absorption was small due to the small size and high reflectivity of the system and thus did not model it. Although the report claims that no photons were timed out, the mean age and surfaces for all of the photons are reported as infinity. Thus, some photons, less than 50 out of a million $(<0.005 \%)$, were timed out or trapped in the crystal. Likewise, the reported uncertainties of $0.00 \%$ are actually non-zero values, but smaller than the format chosen for the DETECT output $(<0.005 \%)$.

Portion of DETECT output file with light collection efficiency report.

\author{
Simulation Report \\ Photons Simulated: $\quad 1000000$ \\ Photons Counted: $\quad 81.39 \%$ ( $0.00 \%)$ \\ Photons Escaped: $\quad 0.00 \%$ \\ Photons Bulk Absorbed: $\quad 0.00 \%$ \\ Photons Surface Absorbed: $18.61 \%(0.00 \%)$ \\ Photons Timed Out: $\quad 0.00 \%(0.00 \%)$ \\ Photons Shifted (Counted): $0.00 \%$ \\ Photons Shifted (All): $\quad 0.00 \%$ \\ Mean Age (Counted): $\quad 846.4$ ( 0.9 ) \\ Mean Age (All): Inf \\ Mean Surfaces (Counted): $\quad 13.5(0.0)$ \\ Mean Surfaces (All): Inf \\ Total Counted Photons: 813870
}




\section{APPENDIX C PHOTODIODE CALIBRATION TECHNIQUE AND DATA}

The Hamamatsu S3590-03 silicon PIN photodiode was calibrated by Hamamatsu hefore it was shipped to us. The calibration was performed using the electronics schematic shown in Fig. C.1 by first measuring the response of a NIST traceable, standard photodiode and then comparing the response of our photodiode to the known characteristics of the standard. The response of the photodiode was recorded as radiant sensitivity $R(\lambda, T, \theta)$, which is the current induced by an incident photon beam of known power at wavelength $\lambda$, temperature $T$, and angle $\theta$. To accurately characterize the photodiode response, measurements were made in $10 \mathrm{~nm}$ increments from 360 to $400 \mathrm{~nm}$ and in $20 \mathrm{~nm}$ increments from 400 to $1100 \mathrm{~nm}$. The response of silicon photodiodes has some fine structure below $400 \mathrm{~nm}$ because the index of refraction for silicon has a pronounced peak. around $380 \mathrm{~nm}$ that results in a significant reflection of incident photons. Thus, smaller increments were used below $400 \mathrm{~nm}$ to characterize the photodiode response. The calibration was performed at room temperature with the photon hear perpendicularly incident on the photodiode, thus $R\left(\lambda, 25^{\circ} \mathrm{C}, \theta=0^{-}\right)$was measured and the results appear in Table $C .1$. As mentioned above, $R\left(\lambda, 25^{\circ} \mathrm{C}, \theta=()^{\circ}\right)$ shows some fine structure below $400 \mathrm{~nm}$ while increasing from $125 \mathrm{~mA} / \mathrm{W}$ at $360 \mathrm{~nm}$ to a peak value of $597 \mathrm{~mA} / \mathrm{W}$ at $960 \mathrm{~nm}$. Above $960 \mathrm{~nm}, \mathrm{R}\left(\lambda, 25^{\circ} \mathrm{C}, \theta=0^{\circ}\right)$ decreases abruptly to $72 \mathrm{~mA} / \mathrm{W}$ at $1100 \mathrm{~nm}$ as the photon energy approaches the band gap of silicon (about $1.11 \mathrm{eV}$, or about $1120 \mathrm{~nm}$ ). The radiant sensitivity over the range of 360 to $700 \mathrm{~nm}$ was subsequently used in Chapters III and IV with the CsI(TI) emission spectrum to calculate the wavelengthaveraged quantum efficiency. 


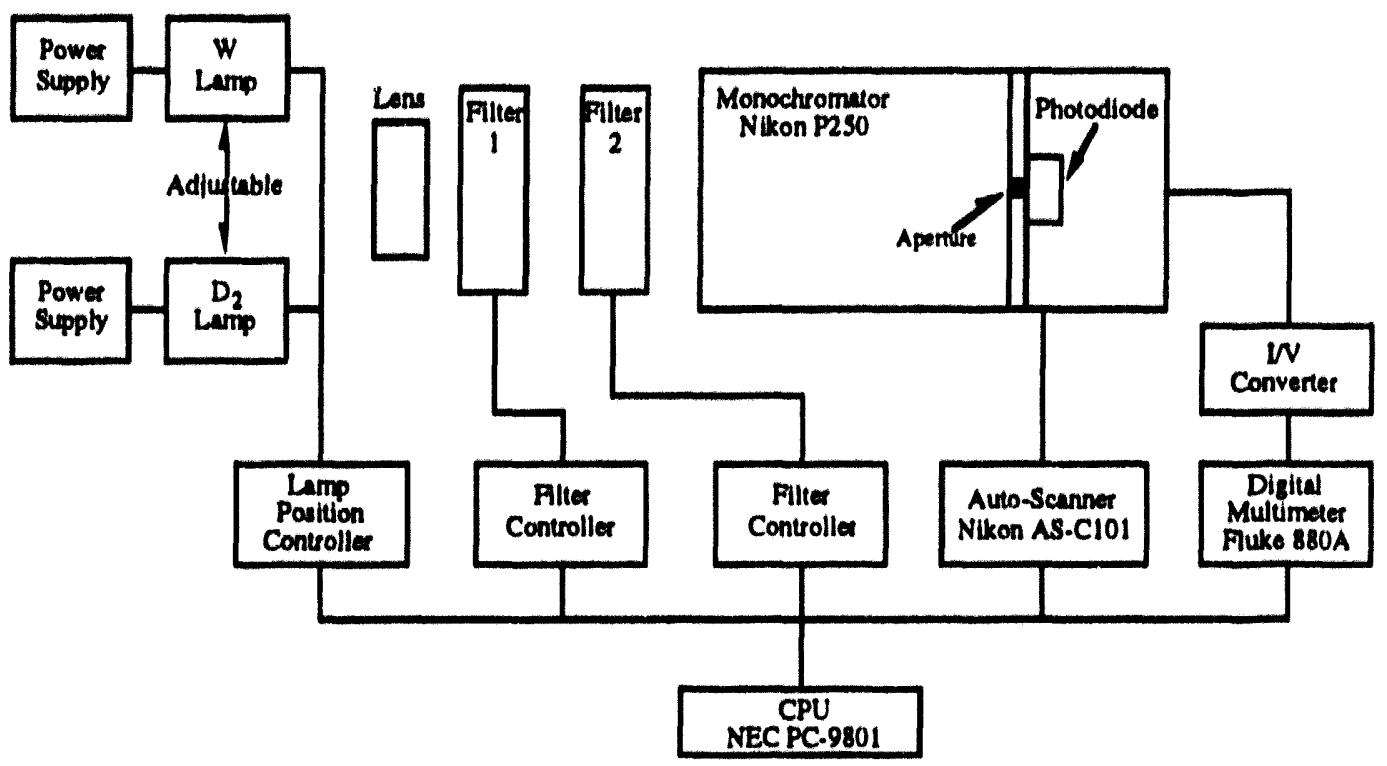

Figure C.1: Photodiode calibration method schematic.

Table C.1: Radiant sensitivity of calibrated Hamamatsu S3590-03 silicon PIN photodiode at room temperature.

\begin{tabular}{|c|c|}
\hline $\begin{array}{c}\text { Wavelength } \\
(\mathrm{nm})\end{array}$ & $\begin{array}{c}\mathrm{R}\left(\lambda, 25^{\circ} \mathrm{C}, \theta=0^{\circ}\right) \\
(\mathrm{mANW})\end{array}$ \\
\hline 360 & 125 \\
\hline 370 & 128 \\
\hline 380 & 139 \\
\hline 390 & 155 \\
\hline 400 & 168 \\
\hline 420 & 193 \\
\hline 440 & 215 \\
\hline 460 & 235 \\
\hline 480 & 253 \\
\hline 500 & 272 \\
\hline 520 & 290 \\
\hline 540 & 305 \\
\hline 560 & 321 \\
\hline 580 & 338 \\
\hline 600 & 352 \\
\hline 620 & 368 \\
\hline 640 & 384 \\
\hline 660 & 398 \\
\hline 680 & 412 \\
\hline 700 & 426 \\
\hline
\end{tabular}

\begin{tabular}{|c|c|}
\hline $\begin{array}{c}\text { Wavelength } \\
(\mathrm{nm})\end{array}$ & $\begin{array}{c}\mathrm{R}\left(\lambda, 25^{\circ} \mathrm{C}, \theta=0^{\circ}\right) \\
(\mathrm{mA} W)\end{array}$ \\
\hline 720 & 441 \\
\hline 740 & 454 \\
\hline 760 & 469 \\
\hline 780 & 486 \\
\hline 800 & 497 \\
\hline 820 & 512 \\
\hline 840 & 525 \\
\hline 860 & 539 \\
\hline 880 & 553 \\
\hline 900 & 567 \\
\hline 920 & 580 \\
\hline 940 & 592 \\
\hline 960 & 597 \\
\hline 980 & 579 \\
\hline 1000 & 532 \\
\hline 1020 & 441 \\
\hline 1040 & 320 \\
\hline 1060 & 191 \\
\hline 1080 & 117 \\
\hline 1100 & 72 \\
\hline
\end{tabular}




\section{APPENDIX D}

\section{INSTRUMENTATION IMPULSE RESPONSE FUNCTION AND CSI(TI) \\ RISE AND DECAY COMPONENT RESULTS FOR MODIFIED BOLLINGER-THOMAS METHOD}

\section{D.1 Impulse Response Function}

The non-linear least squares fitting program used to analyze the luminescence timing spectra of CsI(Tl) was written by Stephen E. Derenzo of Lawrence Berkeley Laboratory and is described in [DER93]. As described in Section 2.3.1, the program deconvolves the instrumentation impulse response function, $G(t)$, out of the observed timing spectrum to arrive at the true luminescence spectrum of $\mathrm{CsI}(\mathrm{TI}), \mathrm{I}(\mathrm{t})$. The instrumentation impulse response function, in Eqn. (2.22), is expressed as the sum of I exponential triangles:

$$
\begin{aligned}
& G(t)=\sum_{i=1}^{I} G_{i}(t) \\
& G_{i}(t)= \begin{cases}\frac{b_{i}}{u_{i}+v_{i}} e^{\left(t-s_{i}\right) / u_{i}} & t<s_{i}, \\
\frac{b_{i}}{u_{i}+v_{i}} e^{-\left(t-s_{i}\right) / v_{i}} & t \geq s_{i}\end{cases}
\end{aligned}
$$

where $b_{i}, u_{i}, v_{i}$, and $s_{i}$ are the area, rise time constant, decay time constant, and time shift of $\mathrm{i}^{\text {th }}$ triangle. The fitting program normalizes the impulse response function to unit area:

$$
\int_{-\infty}^{+\infty} G(t) d t=\sum_{i=1}^{I} b_{i}=1
$$

with the result that the area under the first triangle can be computed by

$$
b_{1}=1-\sum_{i=2}^{1} b_{i} .
$$

Additionally, there is no time shift for the first triangle, $s_{1}=(0.0$. The input file pos.par that the fitting program reads $G(t)$ from is listed in the box below. Note that all of the step sizes 
are zero because the impulse response function parameters are not varied during the fit. Note also that $s_{1}$ and $b_{1}$ are predetermined and thus not listed.

\begin{tabular}{|c|c|c|c|}
\hline & parameter & value & step \\
\hline 1 & $v_{1}$ & 1.006893438323 & 0.00 \\
\hline 2 & $u_{1}$ & 0.846863739190 & 0.00 \\
\hline 3 & $b_{2}$ & 0.487008216405 & 0.00 \\
\hline 4 & $s_{2}$ & 0.845027781308 & 0.00 \\
\hline 5 & $v_{2}$ & 1.035099447590 & 0.00 \\
\hline 6 & $\mathrm{u}_{2}$ & 0.345945937184 & 0.00 \\
\hline 7 & $b_{3}$ & 0.00 & 0.00 \\
\hline 8 & $s_{3}$ & 0.00 & 0.00 \\
\hline 9 & $v_{3}$ & 0.00 & 0.00 \\
\hline 10 & $\mathrm{u}_{3}$ & 0.00 & 0.00 \\
\hline 11 & $b_{4}$ & 0.00 & 0.00 \\
\hline 12 & $s_{4}$ & 0.00 & 0.00 \\
\hline 13 & $v_{4}$ & 0.00 & 0.00 \\
\hline 14 & $\mathrm{u}_{4}$ & 0.00 & 0.00 \\
\hline 15 & $b_{5}$ & 0.00 & 0.00 \\
\hline 16 & $s_{5}$ & 0.00 & 0.00 \\
\hline 17 & $v_{5}$ & 0.00 & 0.00 \\
\hline 18 & $\mathrm{u}_{5}$ & 0.00 & 0.00 \\
\hline 19 & $b_{6}$ & 0.00 & 0.00 \\
\hline 20 & $s_{6}$ & 0.00 & 0.00 \\
\hline 21 & $v_{6}$ & 0.00 & 0.00 \\
\hline 22 & $\mathrm{u}_{6}$ & 0.00 & 0.00 \\
\hline 23 & $b_{7}$ & 0.00 & 0.00 \\
\hline 24 & $s_{7}$ & 0.00 & 0.00 \\
\hline 25 & $v_{7}$ & 0.00 & 0.00 \\
\hline 26 & $u_{7}$ & 0.00 & 0.00 \\
\hline
\end{tabular}

\section{D.2 Output File Listings}

Recalling that the program is fitting for $I(t)$ with a sum of $J$ exponential components:

$$
I(t)=\sum_{j=1}^{J} \frac{a_{j}}{\tau_{j}} e^{-t / \tau_{j}},
$$


where $a_{j}$ and $\tau_{j}$ are the integrated luminescence and rise or decay time constant of the $\mathrm{i}^{\text {th }}$ component, respectively. In this representation, an exponential rise will manifest itself as a negative integrated luminescence as the rise effectively takes away a fraction of the area under the decay component that it is associated with. The program normalizes $I(t)$ to

$$
\int_{0}^{\infty} I(t) d t=\sum_{j=1}^{j} a_{j},
$$

such that $a_{j}$ represents the fraction of the light emitted by the $j^{\text {th }}$ component. The normalization also enables the integrated luminescence of the first component to be calculated by

$$
a_{1}=1-\sum_{j=2}^{J} a_{j} .
$$

One of the output files, stream.out, from the fitting program lists most of the important information regarding the fit. The following is a compilation of all of these files for the results presented in Chapter V. An example of the section of the output file that lists the fit parameters and their uncertainties $\left(-40^{\circ} \mathrm{C}, 50 \mu \mathrm{s}\right.$ TAC range $)$ appears in the box below. The background (bkg) or chance coincidence rate multiplied by the bin width was held constant for all long TAC range data, as explained is Section 5.3.1. The fixed delay between the TAC start and stop signals is reported as $\varphi_{0}$. Parameters that have step values of 0.0 were held constant during the fit, except for parameter $a_{1}$ which is calculated by Eqn. (D.6). The order of the rise and decay components in the output file is somewhat random. Usually, the short primary decay component (reported as $\tau_{1}$ in Chapter $V$ ) is first and the other rise and decay times are in ascending order after that with the ultra-fast component last. In this particular file, the rise, long primary decay, and ultra-fast components happen to be components 3,4 , and 5 , respectively. However, this pattern is often broken. The $\pm \sigma$ value that appears is the average of the $-\sigma$ and $+\sigma$ values that are determined by the program. Note that the decay time of the ultra-fast component is always held constant at $10 \mathrm{ps}$, consistent with the findings of Deich, et al. [DEI89], while its 
fraction is allowed to vary. If the ultra-fast decay time was not held constant, the program converged slowly and the resulting decay time would vary from 10 to 500 ps randomly. This variation is not surprising since the FWHM of the impulse response function is $5(X)$ ps. We also observed that the two additional decay components that appear at -31$)$ and $-100)^{\circ} \mathrm{C}$ were difficult to resolve when the ultra-fast decay time was allowed to vary.

\begin{tabular}{|c|c|c|c|c|c|c|}
\hline & \# & value & step & -0 & +0 & \pm 0 \\
\hline bkg & 27 & 16.4000000 & $0.0000 \times 00$ & $0.0 \times O O O C X)$ & $0.0(0) 00000$ & $0.00(x)(x)(x)$ \\
\hline 4 & 28 & 20.418401 & 0.100000 & $0.00 \times 1000)$ & $0.0000000)$ & 0.00000000 \\
\hline$a_{1}$ & 29 & 0.791192 & 0.0000000 & 0.0000000 & $0.0(000000$ & $0.00(0)(X)$ \\
\hline & 30 & 1771.085869 & 10.000000 & -64.213826 & 61.385539 & 62.799683 \\
\hline$a_{2}$ & 31 & 0.002994 & $0.0000000)$ & $0.0 \%(0) 00)$ & $0.000(0) 0$ & $0.00000(x)$ \\
\hline & 32 & 4.909857 & 0.01000000 & $0 .(10 X)(O) X)$ & $0.0 \times 0 O(X) 0)$ & $0.00(00)(x)$ \\
\hline & 33 & -0.164663 & $0.0(00100)$ & -0.024146 & 0.019169 & 0.021657 \\
\hline - & 34 & 460.248257 & 10.0000000 & $-33.6(14481$ & $37.3591(1) 4$ & 35.481793 \\
\hline$a_{4}$ & 35 & 0.3675 & 0.005000 & $-(0.012584$ & 0.012993 & 0.012789 \\
\hline$\tau$ & 36 & 11721.254495 & 10.(XKOOOOX) & -602.946543 & 660.994660 & 631.9711602 \\
\hline & 37 & 0.002916 & $0.0 \%(O O O O O)$ & $0 . \cos (\cos )$ & 0.0 (X)K(K)(K) & $0.0(0)(0)(x)$ \\
\hline & 3 & $0.01000 x$ & $0.06 \times(0 \times 0)$ & $0.0(X)(X)(X)$ & (1.)(X)(X)(X) & $0.00000(x)$ \\
\hline
\end{tabular}

The measured timing spectrum that was heing fit is listed as the data file and appears either as temperature_short.dat or as temperature_long.dat. The files with short correspond to data acquired with the $1 \mu \mathrm{s}$ TAC range, while long corresponds to the $20 \mu \mathrm{s}$ $\mathrm{TAC}$ range data for $\mathrm{T}>-200^{\circ} \mathrm{C}$ and the $50 \mu \mathrm{s} \mathrm{TAC}$ range data for $\mathrm{TS}-20{ }^{\circ} \mathrm{C}$. The Elapsed time in stream and Elapsed time in shore entries refer io the microprocessor time used to fit the data and to determine the parameter standard deviations, respectively. The reduced $\chi^{2}$ can be determined by dividing the Best fit chi squared result hy the number of hins (listed on the line with signal counts...) minus the number of free parameters and is tabulated in Table D. 1 for each file at the end of this appendix. 
Compilation of stream.dat output files for the Solon Technologies, Inc. crystal in the range of $-1000^{\circ} \mathrm{C} S \mathrm{TS}+50^{\circ} \mathrm{C}$ and at $23^{\circ} \mathrm{C}$ and $10^{\circ} \mathrm{C}$ for the Bicron Corporation, Hortha Crystal Products, and Rexon Components, Inc. crystals.

data file: 31$)^{\circ} \mathrm{C}$ _long.dat

impulse file: pos.par

maximum counts $14464.0 X X X X X)$ at $49.92(X X X)$ ns - hin 26

signal counts $1160860.56(0 \times(X)$ over 335 hins

time per hin (ns): 16.639799

Elapsed time in stream= $1 \mathrm{sec}$

Best fil chi squared $=373.791645$

Elapsed time in shore $=6$ sec

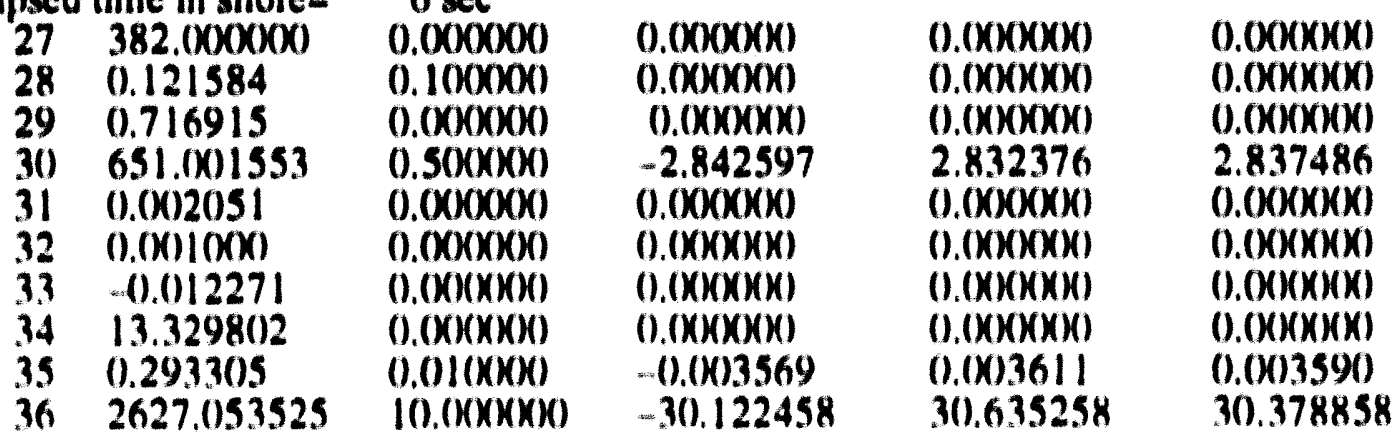

data file: $50^{\circ} \mathrm{C}_{-}$shorn dat

impulse file: poss.par

maximum counts 1638 . $(X X X X X X)$ at $54.1(124(X X) \mathrm{ns}-$ hin 133

signal counts 99$) 1407.92(X X X)$ over 376 bins

time per hin (ns): 0.858806

Elapsed time in stream $=\quad 1 \mathrm{sec}$

Best fit chi squared= 523.976839

Elapsed time in shore $=28 \mathrm{sec}$

\begin{tabular}{|c|c|c|c|c|}
\hline 1175 & & & & \\
\hline 2320965 & $0.1(1)(X 0) 0)$ & $\begin{array}{l}0 .(X)(X X Y Y) \\
0\end{array}$ & $\begin{array}{l}(0 .(X)(O)(X) \\
\text { (X) }\end{array}$ & $\begin{array}{l}0.0()(X)(X) \\
0\end{array}$ \\
\hline 0.716915 & $0 .(X)(X \times) 0$ & $0.0(X)(X X)(0)$ & (). (X)(א)(X)1 & $0.00(x)(x)$ \\
\hline 628.361095 & $50.0(X) \times(X)()$ & -2.793267 & $2.80184(14$ & $2.80(0) .36$ \\
\hline $0 .(0) 2051$ & $0 .(X)(X) 10$ & $-0.0(0)(x) 58$ & (1).(x)(x)sy & ()$.(X)(X) 58$ \\
\hline$(0.1)(1)](x)$ & $0 .(x)(x)(0) 0)$ & ()$.(X)(X)(X)()$ & $(1) .(x)(0)(x)(x)$ & (). ()()$(X)(X)$ \\
\hline -1) & $0.010 \times(0)$ & $-(1.0(6) 287$ & $0 .(x)(1281$ & 0.000284 \\
\hline 13,329802 & 0.4()$(x)(0) 0)$ & $-(0.445625$ & 0.461523 & 0.453574 \\
\hline (0.293305 & $0.0 \times(X)(0)$ & (). (X)KXYKO) & $(0 .(x)(1000)$ & $0.0)(x)(x)$ \\
\hline 2627.053525 & $0 .(X)(X)()()$ & ().(X)(XXY)(O) & $(0 .(x)(x)(x)()$ & (). (o) ofock \\
\hline
\end{tabular}


data file: $40^{\circ} \mathrm{C}$ _long.dat

impulse file: pos.par

maximum counts $272.0 \times 0 \times(X)$ at $66.56(000) \mathrm{ns}-$ hin 27

signal counts $37149 .(x \times O X X X)$ over 335 hins

lime per bin (ns): 16.639799

Elapsed time in stream= 1 sec

Best fit chi squared $\mathbf{3 6 6 . 4 5 5 4 1 0}$

Elapsed time in shore $=16$ sec

\begin{tabular}{|c|c|c|c|c|}
\hline moono & 10 ster & & & \\
\hline $2 . \operatorname{lom}^{2}$ & 0.1日h & 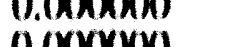 & D. & 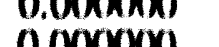 \\
\hline & 0.1 & 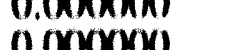 & 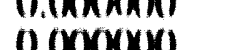 & О.ОкКки) \\
\hline 674.924638 & 0 sovero & $0.710(1)$ & 3698531 & 271486100 \\
\hline $0 .(K) 2256$ & YOY00 & o grooxoy) & $00 \times 0 \times 0 \times 0)$ & $10 \times 0 \times \times \times 0$ \\
\hline $0.010 \times 1 \times 0$ & $0.00 \times \times(x)$ & $0.0 \times \times \times(\times) \times)$ & $0.0 \times \times X \times \times K)$ & (0.0XXXX)XX) \\
\hline-0.013728 & $0.0 \times 1 \times \times \times \times)$ & $0.0 \times \times \times \times \times)$ & $0 .(\times \times \times \times \times)$ & $0.0 \times \times \times(X)$ \\
\hline 13.825496 & (). (XKKXXX) & (). $($ XXXYXX) & $0.0 \times \times \times \times \times)$ & (0.(XXXXXX) \\
\hline 0.361569 & $(0,(X)](X X)$ & -0.030496 & 0.032729 & 0.031612 \\
\hline 2964.73425 & 10.0KKKXXK) & $=268.514240$ & $312.9464 \times 4$ & 290.725362 \\
\hline
\end{tabular}

data file: $41^{\circ} \mathrm{C}$.short.dat

impulse file: pos.par

maximum counts 45.(XXXXXXX) at $1(19.07(X X X) \mathrm{ns}-$ hin 197

signal counts 2286()$,(X)(0 \times(X)$ over 376 hins

lime per hin (ns): ().8588()6

Elapsed time in stream = $(1)$ sec

Best fil chi squared 357,469558

Elapsed time in shore $=28 \mathrm{sec}$

\begin{tabular}{|c|c|c|c|c|}
\hline 6.328352 & (). $011(x \times x)$ & (). $(x \times \times \times x \times)$ & $0 .(\times \times \times \times \times)$ & (). $(x)(x \times(x)$ \\
\hline 2.219627 & $0.10 \times \times \times \times)$ & $0 .(X \times X \times \times X)$ & (1). $(x \times)(x \times)$ & ().(XXXXXX) \\
\hline (0.649904 & (0.0XXXXX) & $0,(X \times X \times \times \times)$ & $0 .(x \times \times \times \times \times)$ & 0. $(x \times \times) \times x$ \\
\hline 688060$)$ & $50.0 \times \times \times \times \times \times)$ & 24.268964 & $25.358721)$ & 24.81 .3842 \\
\hline 2256 & $0.0 \times \times X) 10$ & $-(1) .(x \times) 387$ & $0 .(x \times 1418$ & $0.0(x) 41) 2$ \\
\hline$(0.0) /(\times \times \times)$ & $0.0 \times \times \times \times \times)$ & (1). $(x \times \times \times \times \times)$ & 0), (x)xxxx) & $0 .(6 \times \times \times)(x)$ \\
\hline 3728 & $(1 .(x))(x)$ & $=() .(X) 2228$ & $0 .(x) 1894$ & $0 .(1 \times 12061$ \\
\hline 5497 & $(0,4(\times \times \times \times)$ & -2.480523 & 3.168493 & $2.8245(1) 8$ \\
\hline 564 & ().(XXXXXX) & (). (XXXXXX) & $(0 .(x \times \times \times \times X)$ & $(0 .()(K)(X)(X)$ \\
\hline 35425 & ().(XXXXX)(1) & (). $(x \times \times \times \times \times)$ & (). (XXXXXXX) & $0 .(1) \times(x)(x)$ \\
\hline
\end{tabular}


data file: 30tC long.dat

impulse nile: pos.par

maximum counts 274.(XXXXXX) at 66.56(XXX) ns - hin 27

signal counts 25837 , (XOXXXX) over 335 hins

lime per hin (ns): 16.639799

Flapsed time in stream= () sec

Best fit chi wquared 307.542599

Elapsed lime in shores 14 sex

\begin{tabular}{|c|c|c|c|c|}
\hline $27976(x)(x)$ & $0 .(x \times x \times x \times)$ & $0 .(\times \times \times \times \times X)$ & (1) $(x \times \times \times \times \times)$ & $0 .(X \times X \times 1 \times)$ \\
\hline 0.460794 & $0.1(\times \times \times \times)$ & $(0,(X \times \times \times X)$ & (1). $(x \times \times \times \times \times)$ & (). (KXXXKX) \\
\hline$(1.65556)$ & (). (XXXXXX) & (1): & 1), (x)XXX) & (). (КХХХХХ) \\
\hline 718.867927 & $0.5(\times \times \times \times)$ & -24.069174 & $23.7497(x)$ & $23.9 \times 19441$ \\
\hline$(0,(X) 1898$ & 0.(XXXYXXX) & ().(XXXYXX) & (). $(\times \times \times \times \times)$ & $0 .(X \times \times \times \times)$ \\
\hline$(1,(1)(x \times X)$ & (). (XXXXXX) & $(0 .(X X X \times X X)$ & $0 .(\times \times \times \times \times X)$ & ().(XXXXXX) \\
\hline$(1.0113544$ & $0 .(\times \times \times \times \times X)$ & (). (XXXXXX) & $0 .(\times \times \times \times \times \times)$ & $0 .(X \times X \times K)$ \\
\hline $13.49 \times 1437$ & $0 .(\times \times \times \times \times X)$ & $0 .(\times \times \times \times \times X)$ & (1), (XXXXX)( & $0 .(\times \times \times \times \times)$ \\
\hline 0.3560185 & $0 .(x) 5(x \times)$ & $-1) .024161$ & (0.0254.32 & 0.024746 \\
\hline 1116.814525 & 10 (X)XXXXX) & 214.289963 & $24(1.2201365$ & 227.255164 \\
\hline
\end{tabular}

data file: 3$)^{a} \mathrm{C}_{\text {sn }}$ shurn dal

impulse file: poss par

maximum counts $48 .(x \times x \times(x)$ al $9(1) .174(x)) \mathrm{ns}-$ hin 175

vignal counts $23118 .(X X X X X X)$ over 376 hins

lime per hin (ns): ().8588(K

lilapsed lime in streum 1 sec

Hest fit chi squared 326.56841 s

lilansed lime in shore $3(1)$ sec

\begin{tabular}{|c|c|c|c|c|}
\hline $3,2(x), 366$ & (0.) $1(x \times x)$ & $(1)(x \times x \times \times \times)$ & (). $(x \times x \times x)$ & (): (x)(x)(x) \\
\hline 2.442473 & (1). $1(x \times \times \times)$ & ().(XXXYXX) & (1). (XXXXX) & $0 .(1)(x)(x)$ \\
\hline 0.655561 & $(1)(x \times \times \times \times)$ & $(1)(x \times \times \times \times x)$ & (1). $(x \times x \times x \times)$ & $0.0)(x \times)(x)$ \\
\hline 242876 & $50 .(X) \times K X)(X)$ & $-|8.548(1) 2|$ & 19.329773 & 18.938897 \\
\hline (1)(x)1898 & $0,(x \times(x) 10$ & $(1) .(x \times), 321$ & $(1)(x \times) 341$ & $0.0(x) 3.31$ \\
\hline$(0,0)(1 \times \times \times)$ & $0 .(x \times \times \times \times)$ & ()$(x \times x \times x)$ & (). (XXXXX) & ().(5)(XXX) \\
\hline ().(1)1.3544 & $(0 .(x \times x) 10$ & $-(1)(x) 17(1) 7$ & ()$,(x) 152.5$ & $0 .(10) 1616$ \\
\hline $13.49(159(1)$ & $0.4(x \times X \times)$ & $=1.968859$ & 2.332683 & $2.15(1771$ \\
\hline$(1,3.56(1) 8.5$ & (). (XXXXXXX) & (). $(x \times X \times X X)$ & (). (XKXXXX) & $(0 .(x)(x)(x)$ \\
\hline 3116.819525 & (). (XXXXXX) & $0 .(X \times X \times X \times)$ & (). $(x \times x \times x)$ & $0 .(x)(x) \times)$ \\
\hline
\end{tabular}




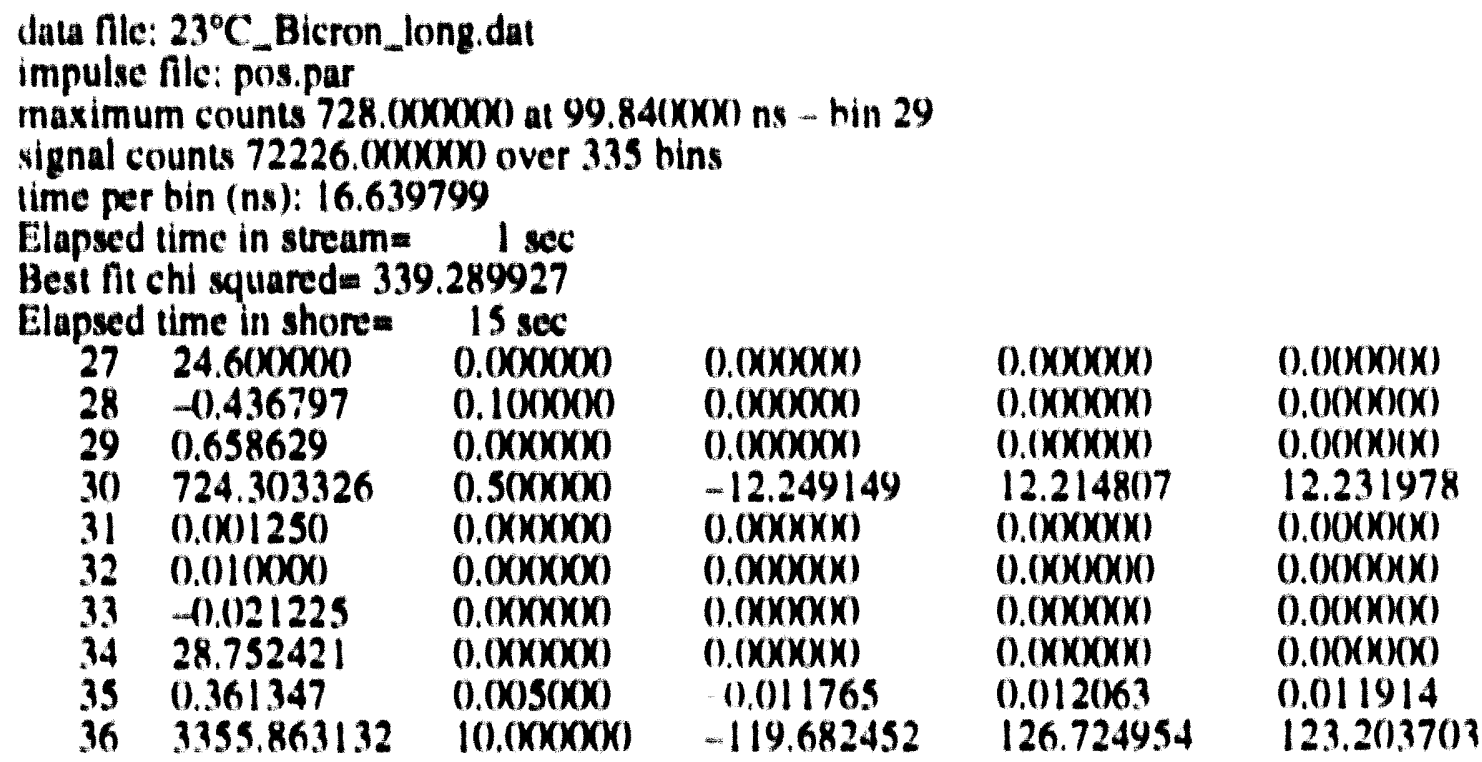

data fille: $23^{\circ} \mathrm{C}$ _Bicron_short.dat

impulse file: pos.par

maximum counts $59 .(X \times X \times(X)$ at $74.716(X X) \mathrm{ns}=$ hin 1.56

vignal counts 26781 . (XXXXXX) over 375 hins

lime per hin (ns): (0.858805

Elapsed time in stream $=\quad 0 \mathrm{sec}$

Best fit chi squared $=4(19.960613$

Elapsed time in shore $=30 \mathrm{sec}$

\begin{tabular}{|c|c|c|c|c|}
\hline 3.652775 & $0.010 \times \times \times 0$ & $0.0 \times \times \times \times \times x)$ & $0 .(x)(x)(x)()$ & $0.0(x)(x)(x)$ \\
\hline $2.4(14462$ & $0.010 \times 0 \times 0$ & $0 .(X X X \times) \times()$ & $(0 .(x \times x \times x)$ & $0.0(x)(x)(x)$ \\
\hline 0.658629 & (1). $(X \times X \times X \times X)$ & $0 .(x \times \times \times \times x)$ & $0 .(x \times \times \times \times x)$ & $0.0(x)(x)(x)$ \\
\hline 676.888313 & $50 .(x \times \times \times \times \times)$ & 22.562825 & 23.364316 & 22.963 .571 \\
\hline$-11,1121225$ & $0.0 \times \times) 5(00)$ & $-(0 .(0) 3(0) 81$ & $(1,1 \times 1) 2660$ & (1).001)2871 \\
\hline 28.752426 & $1,(x \times x \times x \times)$ & -4.0332119 & 4.674952 & 4.354080 \\
\hline $0.0 \times 11250$ & $0 .(x) 10(0) 0$ & $-1) .(1)(x) .312$ & $(0.1 \times(x) 311)$ & $0.0(0)(1311$ \\
\hline$x \times(x)$ & (1). $1 \times \times \times \times \times \times)$ & (). $(x \times x \times x \times)$ & (1), $0 \times \times \times \times X \times)$ & (1). $(1)(10 \times)(x)$ \\
\hline (1).361.347 & (). $(x \times \times \times \times \times \times)$ & $0.0 \times \times \times \times \times(0)$ & (1). $(x \times x \times x \times x)$ & $0.0(x)(x)(x)$ \\
\hline 3355.863132 & $0 .(x)(x)(x) 0$ & $0 .(x)(x)() 0$ & $(1)(x)(x)(x)()$ & $0 .(1)(x)(x)(x)$ \\
\hline
\end{tabular}


data file: $23^{\circ} \mathrm{C}$ _Horiba_long.dat

impulse lile: pos.par

maximum counts $16(1)$. (XXXXXX) at $99.84(X K X)$ ns hin 29

signal counts 175529.(14)(KX)(O) over 335 hins

lime per hin (ns): 16.6.39799

lilapsed time in stream $=1$ sec

Best lit chi syuared $=382.655988$

Blansed time in shore $=11$ sec

\begin{tabular}{|c|c|c|c|c|}
\hline $81 .($ XXKXK) & $0 .(X X \times \times X X)$ & (1). $(x \times x \times x \times)$ & $0.1 \times \times \times \times \times x)$ & $0 .(x)(x)(x)$ \\
\hline (). 299257 & (1). $1(x \times x \times x)$ & (1). (XXXXXX) & (1). $(X X X \times X X)$ & $0 .(x)(x)(x)$ \\
\hline 1).655.363 & $(0,(x \times \times) \times x)$ & $0.1 \times(X) \times(X)$ & (). (XXXXXX) & $0 .(1)(x)(x)$ \\
\hline 679.283864 & $0.5 i \times \times \times \times)$ & 8.517545 & 8.499696 & 8.5118646 \\
\hline (1).(x)1647 & $0 .(x \times x \times x)$ & (I.)(XXXXXX) & $0 .(X \times X) \times X)$ & $0 .(x)(x)(x)$ \\
\hline$(1,0) 110(x)(x)$ & $0 .(x \times x \times x \times)$ & (). (XXXXXX) & (). $(x \times X \times X)$ & $0 .(x)(x)(x)$ \\
\hline (1).11)8362 & $0 .(X X X X X X X)$ & (1). $(x \times x \times x)$ & ()., (XXXXXX) & $0.0)(x)(x)$ \\
\hline 19.621195 & $0 .(x \times x \times x \times)$ & $0 .(X \times X \times X \times)$ & (). (XXXXXX) & (1.)(X)(X)(X) \\
\hline 0.361 .352 & (1). (x) $5(x)$ & 1).(x)18.552 & $0 .(x) 8711)$ & $0.1 \times 186.31$ \\
\hline 3344.1 & $10 .(x \times x \times x \times x)$ & $94.7(19) 267$ & 98.9416811 & 96.82547 \\
\hline
\end{tabular}

dala lile: $23^{\circ} \mathrm{C}$ Horiha_short dal

impulse fi':: pus.par

maximum counts $128 .(X X X X X X)$ at $67.845(X X)$ ns hin 140

signal counts 55437 , $($ KXXXX) over 376 hins

lime per hin (ns): $0.8588(6)$

lilapsed time in stream $=\quad 10 \mathrm{sec}$

Best fit chi syuared $=4(1) .8571607$

Blapsed time in shore $=27 \mathrm{sec}$

\begin{tabular}{|c|c|c|c|c|}
\hline 7.627915 & $(0 .() 1(x \times x)$ & 1). $(x \times x \times x)$ & (). (xxXXXX) & $0 .(1)(x)(x)$ \\
\hline $2.2(1) 4885$ & (1. I (XXXXX) & (1). $(x \times x \times x \times)$ & (1).(xxxxxx) & $(1)(x)(x)(x)$ \\
\hline (1).655.36.3 & $(1 .(x \times x \times x)$ & $11 .(x \times x \times x)$ & $0 .(x \times x \times x \times)$ & $(1)(x)(x)(x)$ \\
\hline 644.968552 & $50 .(x \times x \times x(x)$ & $-13.1973(18$ & $1.3 .54(1) 188$ & 13.3687 .48 \\
\hline (). $(x) 16.47$ & $(1)(x \times x) 10$ & $-(1)(x(x) 188$ & $(1)(x \times) 199$ & $(1) .(1)(0) 19.3$ \\
\hline$(1.1) 1(x \times x)$ & $0 .(x \times x \times x)$ & (1). (XXXXXXX) & (1).(XXXKXX) & ()$.(K)(X)(X)$ \\
\hline (1).(1) $\times .362$ & $(0 .())(x \times x)$ & $(0 .(x) 15(1) 5$ & $(0 .(x) 1.387$ & $(0.1(x) 1446$ \\
\hline 19.621195 & $(0.4(x \times \times \times \times)$ & -1.773793 & 1.963551 & 1.8686772 \\
\hline (1). 361352 & (). $(x \times \times \times x)$ & (). (XXXXXX) & (1).(x)xxxx) & $0 .(1)(x)(x)(x)$ \\
\hline 3344.1251198 & $(0 .(x \times x \times x)$ & (). $(x \times x \times x)$ & $0(x) \times x \times x)$ & ()$.(K)(X)(X)$ \\
\hline
\end{tabular}


data file: $23^{\circ} \mathrm{C} \_$Rexon_long.dat

impulse file: pos.par

maximum counts 2174.000000 at $66.560000 \mathrm{~ns}-$ bin 27

signal counts 178718.000000 over 335 bins

time per bin (ns): 16.639799

Elapsed time in stream $=1 \mathrm{sec}$

Best fit chi squared $=369.150420$

Elapsed time in shore $=6 \mathrm{sec}$

$\begin{array}{rlllll}27 & 47.000000 & 0.000000 & 0.000000 & 0.000000 & 0.000000 \\ 28 & 0.231790 & 0.100000 & 0.000000 & 0.000000 & 0.000000 \\ 29 & 0.635091 & 0.000000 & 0.000000 & 0.000000 & 0.000000 \\ 30 & 645.846948 & 0.500000 & -6.214872 & 6.220959 & 6.217915 \\ 31 & 0.002530 & 0.000000 & 0.000000 & 0.000000 & 0.000000 \\ 32 & 0.010000 & 0.000000 & 0.000000 & 0.000000 & 0.000000 \\ 33 & -0.020322 & 0.000000 & 0.000000 & 0.000000 & 0.000000 \\ 34 & 23.683718 & 0.000000 & 0.000000 & 0.000000 & 0.000000 \\ 35 & 0.382701 & 0.005000 & -0.005947 & 0.006007 & 0.005977 \\ 36 & 3246.052128 & 10.000000 & -57.034346 & 58.634592 & 57.834469\end{array}$

data file: $23^{\circ} \mathrm{C} \_$Rexon_short.dat

impulse file: pos.par

maximum counts 110.000000 at $1.717600 \mathrm{~ns}-\operatorname{bin} 72$

signal counts 53694.000000 over 376 bins

time per bin (ns): 0.858806

Elapsed time in stream $=1 \mathrm{sec}$

Best fit chi squared $=388.551076$

Elapsed time in shore $=26 \mathrm{sec}$

$\begin{array}{llllll}27 & 5.612050 & 0.010000 & 0.000000 & 0.000000 & 0.000000 \\ 28 & 2.337553 & 0.100000 & 0.000000 & 0.000000 & 0.000000 \\ 29 & 0.635091 & 0.000000 & 0.000000 & 0.000000 & 0.000000 \\ 30 & 616.925663 & 50.000000 & -12.286581 & 12.590429 & 12.438505 \\ 31 & 0.002530 & 0.000010 & -0.000208 & 0.000219 & 0.000214 \\ 32 & 0.010000 & 0.000000 & 0.000000 & 0.000000 & 0.000000 \\ 33 & -0.020322 & 0.010000 & -0.001690 & 0.001561 & 0.001626 \\ 34 & 23.683789 & 0.400000 & -2.083024 & 2.283042 & 2.183033 \\ 35 & 0.382701 & 0.000000 & 0.000000 & 0.000000 & 0.000000 \\ 36 & 3246.052128 & 0.000000 & 0.000000 & 0.000000 & 0.000000\end{array}$


data file: $23^{\circ} \mathrm{C} \_$long.dat

impulse file: pos.par

maximum counts 5532.000000 at $66.560000 \mathrm{~ns}-$ bin 27

signal counts 679902.440000 over 335 bins

time per bin (ns): 16.639799

Elapsed time in stream $=1 \mathrm{sec}$

Best fit chi squared $=388.767668$

Elapsed time in shore $=5 \mathrm{sec}$

$\begin{array}{llllll}27 & 290.000000 & 0.000000 & 0.000000 & 0.000000 & 0.000000 \\ 28 & -0.237697 & 0.100000 & 0.000000 & 0.000000 & 0.000000 \\ 29 & 0.627492 & 0.000000 & 0.000000 & 0.000000 & 0.000000 \\ 30 & 781.504086 & 10.000000 & -5.355928 & 5.350053 & 5.352991 \\ 31 & -0.016081 & 0.000000 & 0.000000 & 0.000000 & 0.000000 \\ 32 & 22.662072 & 0.000000 & 0.000000 & 0.000000 & 0.000000 \\ 33 & 0.386836 & 0.000500 & -0.004838 & 0.004880 & 0.004859 \\ 34 & 3528.396410 & 10.000000 & -49.048433 & 50.191889 & 49.620161 \\ 35 & 0.001753 & 0.000000 & 0.000000 & 0.000000 & 0.000000 \\ 36 & 0.010000 & 0.000000 & 0.000000 & 0.000000 & 0.000000\end{array}$

data file: $23^{\circ} \mathrm{C} \_$short.dat

impulse file: pos.par

maximum counts 510.000000 at $2.576400 \mathrm{~ns}-$ bin 72

signal counts 307536.160000 over 375 bins

time per bin (ns): 0.858805

Elapsed time in stream $=0 \mathrm{sec}$

Best fit chi squared $=449.966553$

Elapsed time in shore $=32 \mathrm{sec}$

$\begin{array}{llllll}27 & 39.354900 & 0.010000 & 0.000000 & 0.000000 & 0.000000 \\ 28 & 2.572595 & 0.100000 & 0.000000 & 0.000000 & 0.000000 \\ 29 & 0.627485 & 0.000000 & 0.000000 & 0.000000 & 0.000000 \\ 30 & 771.549860 & 10.000000 & -8.062233 & 8.177199 & 8.119716 \\ 31 & -0.016081 & 0.000500 & -0.000606 & 0.000583 & 0.000595 \\ 32 & 22.662070 & 1.000000 & -0.972159 & 1.018096 & 0.995128 \\ 33 & 0.001761 & 0.001000 & -0.000076 & 0.000077 & 0.000076 \\ 34 & 0.010000 & 0.000000 & 0.000000 & 0.000000 & 0.000000 \\ 35 & 0.386836 & 0.000000 & 0.000000 & 0.000000 & 0.000000 \\ 36 & 3528.396410 & 0.000000 & 0.000000 & 0.000000 & 0.000000\end{array}$


data file: $20^{\circ} \mathrm{C} \_$long.dat

impulse file: pos.par

maximum counts 533.000000 at 99.840000 ns - bin 31

signal counts 47967.000000 over 337 bins

time per bin (ns): 16.639800

Elapsed time in stream $=0 \mathrm{sec}$

Best fit chi squared $=381.722387$

Elapsed time in shore $=13 \mathrm{sec}$

$\begin{array}{llllll}27 & 13.150000 & 0.000000 & 0.000000 & 0.000000 & 0.000000 \\ 28 & 16.097429 & 0.100000 & 0.000000 & 0.000000 & 0.000000 \\ 29 & 0.616002 & 0.000000 & 0.000000 & 0.000000 & 0.000000 \\ 30 & 710.062307 & 0.500000 & -14.933906 & 14.899761 & 14.916833 \\ 31 & 0.002086 & 0.000000 & 0.000000 & 0.000000 & 0.000000 \\ 32 & 0.010000 & 0.000000 & 0.000000 & 0.000000 & 0.000000 \\ 33 & -0.019977 & 0.000000 & 0.000000 & 0.000000 & 0.000000 \\ 34 & 24.882950 & 0.000000 & 0.000000 & 0.000000 & 0.000000 \\ 35 & 0.401889 & 0.005000 & -0.013825 & 0.014137 & 0.013981 \\ 36 & 3261.308380 & 10.000000 & -116.346692 & 123.463118 & 119.904905\end{array}$

data file: $20^{\circ} \mathrm{C} \_s h o r t . d a t$

impillse file: pos.par

maximum counts 260.000000 at $2.576400 \mathrm{~ns}$ - bin 73

signal counts 139080.880000 over 376 bins

time per bin (ns): 0.858806

Elapsed time in stream $=3 \mathrm{sec}$

Best fit chi squared $=393.274102$

Elapsed time in shore $=30 \mathrm{sec}$

$\begin{array}{llllll}27 & 12.746701 & 0.010000 & 0.000000 & 0.000000 & 0.000000 \\ 28 & 2.619480 & 0.100000 & 0.000000 & 0.000000 & 0.000000 \\ 29 & 0.616002 & 0.000000 & 0.000000 & 0.000000 & 0.000000 \\ 30 & 686.075235 & 50.000000 & -9.415592 & 9.584612 & 9.500102 \\ 31 & 0.002086 & 0.000010 & -0.000115 & 0.000118 & 0.000116 \\ 32 & 0.010000 & 0.000000 & 0.000000 & 0.000000 & 0.000000 \\ 33 & -0.019977 & 0.010000 & -0.001006 & 0.000955 & 0.000980 \\ 34 & 24.882947 & 0.400000 & -1.337819 & 1.420799 & 1.379309 \\ 35 & 0.401889 & 0.000000 & 0.000000 & 0.000000 & 0.000000 \\ 36 & 3261.308380 & 0.000000 & 0.000000 & 0.000000 & 0.000000\end{array}$


data file: $10^{\circ} \mathrm{C} \_$long.dat

impulse file: pos.par

maximum counts 242.000000 at 99.84000() $\mathrm{ns}-\operatorname{hin} 29$

signal counts 23634.000000 over 335 bins

time per bin (ns): 16.639799

Elapsed time in stream $=\quad 0 \mathrm{sec}$

Best fit chi squared $=324.714643$

Elapsed time in shore $=\quad 14 \mathrm{sec}$

\begin{tabular}{|c|c|c|c|c|}
\hline 7.500000 & 0.000000 & 0.000000 & 0.000000 & 0.000000 \\
\hline 0.510990 & 0.100000 & 0.000000 & 0.000000 & 0.000000 \\
\hline 0.610513 & 0.000000 & 0.000000 & 0.000000 & 0.000000 \\
\hline 749.343285 & 0.500000 & -21.551105 & 21.654781 & 21.602943 \\
\hline 0.002781 & 0.000000 & 0.000000 & .000000 & 0.000000 \\
\hline 0.010000 & 0.000000 & 0.000000 & 0.000000 & 0.000000 \\
\hline-0.019428 & 0.000000 & 0.000000 & 0.000000 & 0.000000 \\
\hline 25.482366 & 0.000000 & 0.000000 & 0.000000 & 0.000000 \\
\hline 0.406 & 0.005000 & -0.017372 & 0.017841 & 0.017607 \\
\hline 3846 . & 10.000000 & -198.929921 & 217.629256 & 208 \\
\hline
\end{tabular}

data file: $10^{\circ} \mathrm{C}$ _short.dat

impulse file: pos.par

maximum counts 42.000000 at $1.717600 \mathrm{~ns}-\operatorname{bin} 72$

signal counts 20385.000000 over 376 bins

time per bin (ns): (ns): 0.858806

Elapsed time in stream $=\quad 4 \mathrm{sec}$

Best fit chi squared $=375.799605$

Elapsed time in shore $=27 \mathrm{sec}$

\begin{tabular}{|c|c|c|c|c|}
\hline & \\
\hline \multicolumn{5}{|c|}{$\begin{array}{llllll}8 & 2.560320 & 0.100000 & 0.000000 & 0.000000 & 0.000000)\end{array}$} \\
\hline \multicolumn{5}{|c|}{$\begin{array}{llllll}28 & 2.560320 & 0.100000 & 0.000000) & 0.000000 & 0.0000(0) \\
29 & 0.610513 & 0.000000 & 0.000000 & 0.0000000 & 0.000000)\end{array}$} \\
\hline \multicolumn{5}{|l|}{$\begin{array}{ll}30 & 684.887182\end{array}$} \\
\hline \multirow{6}{*}{$\begin{array}{ll}8 & 2.560320 \\
9 & 0.610513 \\
0 & 684.887182 \\
1 & 0.002781 \\
2 & 0.010000 \\
3 & -0.019428 \\
4 & 25.482517 \\
5 & 0.406134 \\
6 & 3846.970546\end{array}$} & 0.000010 & -0.000338 & 0.000365 & 0.000351 \\
\hline & 0.000000 & $0.000000)$ & 0.000000 & 0.000000 \\
\hline & 0.010000 & -0.002791 & 0.002434 & 0.002613 \\
\hline & 0.400000 & -3.531168 & 4.137981 & 3.834574 \\
\hline & 0.000000 & 0.000000 & 0.000000 & 0.000000 \\
\hline & 0.000000 & 0.000000 & 0.000000 & 0.000000 \\
\hline
\end{tabular}


data file: $0^{\circ} \mathrm{C} \_$Bicron_long.dat

impulse file: pos.par

maximum counts 1136.000000 at $133.120000 \mathrm{~ns}-\operatorname{bin} 30$

signal counts 128789.000000 over 334 bins

time per bin (ns): (ns): 16.639798

Elapsed time in stream $=\quad 1 \mathrm{sec}$

Best fit chi squared $=337.685654$

Elapsed time in shore $=\quad 14 \mathrm{sec}$

$\begin{array}{llllll}27 & 37.500000 & 0.000000 & 0.000000 & 0.000000 & 0.000000 \\ 28 & 0.142351 & 0.100000 & 0.000000 & 0.000000 & 0.000000 \\ 29 & 0.618963 & 0.000000 & 0.000000 & 0.000000 & 0.000000 \\ 30 & 836.598097 & 0.500000 & -10.290331 & 10.305252 & 10.297792 \\ 31 & 0.001462 & 0.000000 & 0.000000 & 0.000000 & 0.000000 \\ 32 & 0.010000 & 0.000000 & 0.000000 & 0.000000 & 0.000000 \\ 33 & -0.022588 & 0.000000 & 0.000000 & 0.000000 & 0.000000 \\ 34 & 38.044734 & 0.000000 & 0.000000 & 0.000000 & 0.000000 \\ 35 & 0.402163 & 0.005000 & -0.007711 & 0.007807 & 0.007759 \\ 36 & 4206.207696 & 10.000000 & -99.084148 & 103.350516 & 101.217332\end{array}$

data file: $0^{\circ} \mathrm{C} \_$Bicron_short.dat

impulse file: pos.par

maximum counts 160.000000 at $2.576400 \mathrm{~ns}-$ bin 72

signal counts 98668.200000 over 375 bins

time per bin (ns): 0.858805

Elapsed time in stream $=\quad 1 \mathrm{sec}$

Best fit chi squared $=380.336083$

Elapsed time in shore $=31 \mathrm{sec}$

$\begin{array}{llllll}27 & 8.474895 & 0.010000 & 0.000000 & 0.000000 & 0.000000 \\ 28 & 2.661823 & 0.100000 & 0.000000 & 0.000000 & 0.000000 \\ 29 & 0.618963 & 0.000000 & 0.000000 & 0.000000 & 0.000000 \\ 30 & 856.877881 & 50.000000 & -19.184539 & 19.656983 & 19.420761 \\ 31 & 0.001462 & 0.000001 & -0.000108 & 0.000112 & 0.000110 \\ 32 & 0.010000 & 0.000000 & 0.000000 & 0.000000 & 0.000000 \\ 33 & -0.022588 & 0.010000 & -0.001646 & 0.001513 & 0.001579 \\ 34 & 38.044724 & 0.400000 & -2.518653 & 2.719766 & 2.619210 \\ 35 & 0.402163 & 0.000000 & 0.000000 & 0.000000 & 0.000000 \\ 36 & 4206.207696 & 0.000000 & 0.000000 & 0.000000 & 0.000000\end{array}$


data file: $0^{\circ} \mathrm{C}$ _Horiba_long.dat

impulse file: pos.par

maximum counts 1684.000000 at $116.480000 \mathrm{~ns}-\operatorname{bin} 30$

signal counts 171344.000000 over 335 bins

time per bin (ns): 16.639799

Elapsed time in stream $=1 \mathrm{sec}$

Best fit chi squared $=356.083200$

Elapsed time in shore $=11 \mathrm{sec}$

$\begin{array}{llllll}27 & 46.000000 & 0.000000 & 0.000000 & 0.000000 & 0.000000 \\ 28 & -0.045281 & 0.100000 & 0.000000 & 0.000000 & 0.000000 \\ 29 & 0.625410 & 0.000000 & 0.000000 & 0.000000 & 0.000000 \\ 30 & 778.483947 & 0.500000 & -7.511602 & 7.523783 & 7.517693 \\ 31 & 0.001609 & 0.000000 & 0.000000 & 0.000000 & 0.000000 \\ 32 & 0.010000 & 0.000000 & 0.000000 & 0.000000 & 0.000000 \\ 33 & -0.021464 & 0.000000 & 0.000000 & 0.000000 & 0.000000 \\ 34 & 30.430614 & 0.000000 & 0.000000 & 0.000000 & 0.00000) \\ 35 & 0.394445 & 0.005000 & -0.005609 & 0.005663 & 0.005636 \\ 36 & 4361.776137 & 10.000000 & -82.97() 192 & 85.774557 & 84.372374\end{array}$

data file: $0^{\circ} \mathrm{C} \_$Horiba_short.dat

impulse file: pos.par

maximum counts 134.000000 at $2.576400 \mathrm{~ns}-$ bin 72

signal counts 74649.000000 over 375 bins

time per bin (ns): 0.858805

Elapsed time in stream $=3 \mathrm{sec}$

Best fit chi squared $=397.752181$

Elapsed time in shore $=36 \mathrm{sec}$

$\begin{array}{llllll}27 & 6.344836 & 0.010000 & 0.000000 & 0.000000 & 0.000(0) 00 \\ 28 & 2.553526 & 0.100000 & 0.000000 & 0.000000 & 0.000000 \\ 29 & 0.001609 & 0.000000 & 0.000000 & 0.000000 & 0.000000 \\ 30 & 0.010000 & 0.000000 & 0.000000 & 0.000000 & 0.000000 \\ 31 & -0.021464 & 0.010000 & -0.001521 & 0.001409 & 0.001465 \\ 32 & 30.430754 & 0.400000 & -2.073984 & 2.240781 & 2.157383 \\ 33 & 0.625410 & 0.010000 & -0.001419 & 0.001532 & 0.001475 \\ 34 & 779.467369 & 50.000000 & -16.492456 & 16.942695 & 16.717575 \\ 35 & 0.394445 & 0.000000 & 0.0000(0) & 0.0000(0) & 0.000000 \\ 36 & 4361.776137 & 0.000000 & 0.000000 & 0.000000 & 0.000(0) 0\end{array}$


data file: $0^{\circ} \mathrm{C} \_$Rexon_long.dat

impulse file: pos.par

maximum counts 1865.000000 at $166.400000 \mathrm{~ns}-$ bin 32

signal counts 191696.000000 over 334 bins

time per bin (ns): 16.639798

Elapsed time in stream $=\quad 0 \mathrm{sec}$

Best fit chi squared $=382.508389$

Elapsed time in shore $=10 \mathrm{sec}$

$\begin{array}{llllll}27 & 54.000000 & 0.000000 & 0.000000 & 0.000000 & 0.000000 \\ 28 & 0.037737 & 0.100000 & 0.000000 & 0.000000 & 0.000000 \\ 29 & 0.620716 & 0.000000 & 0.000000 & 0.000000 & 0.000000 \\ 30 & 752.671531 & 0.500000 & -7.278744 & 7.287151 & 7.282947 \\ 31 & 0.002546 & 0.000000 & 0.000000 & 0.000000 & 0.000000 \\ 32 & 0.010000 & 0.000000 & 0.000000 & 0.000000 & 0.000000 \\ 33 & -0.026408 & 0.000000 & 0.000000 & 0.000000 & 0.000000 \\ 34 & 35.641027 & 0.000000 & 0.000000 & 0.000000 & 0.000000 \\ 35 & 0.403146 & 0.005000 & -0.005950 & 0.006005 & 0.005978 \\ 36 & 3880.311543 & 10.000000 & -69.339700 & 71.472441 & 70.406071\end{array}$

data file: $0^{\circ} \mathrm{C} \_$Rexon_short.dat

impulse file: pos.par

maximum counts 150.000000 at $2.576400 \mathrm{~ns}-$ bin 72

signal counts 69939.000000 over 375 bins

time per bin (ns): 0.858805

Elapsed time in stream $=1 \mathrm{sec}$

Best fit chi squared $=367.195278$

Elapsed time in shore $=33 \mathrm{sec}$

$\begin{array}{llllll}27 & 5.839163 & 0.010000 & 0.000000 & 0.000000 & 0.000000 \\ 28 & 2.824215 & 0.100000 & 0.000000 & 0.000000 & 0.000000 \\ 29 & 0.002546 & 0.000000 & 0.000000 & 0.000000 & 0.000000 \\ 30 & 0.010000 & 0.000000 & 0.000000 & 0.000000 & 0.000000 \\ 31 & -0.026408 & 0.000100 & -0.001751 & 0.001633 & 0.001692 \\ 32 & 35.640991 & 1.000000 & -2.077211 & 2.213168 & 2.145189 \\ 33 & 0.620716 & 0.010000 & -0.001639 & 0.001758 & 0.001698 \\ 34 & 754.517500 & 50.000000 & -16.407045 & 16.897012 & 16.652029 \\ 35 & 0.403146 & 0.000000 & 0.000000 & 0.000000 & 0.000000 \\ 36 & 3880.311543 & 0.000000 & 0.000000) & 0.000000 & 0.000000\end{array}$


data file: $0^{\circ} \mathrm{C} \_$long.dat

impulse file: pos.par

maximum counts 617.000000 at $166.400000 \mathrm{~ns}-\operatorname{bin} 34$

signal counts 66800.000000 over 336 bins

time per bin (ns): 16.639799

Elapsed time in stream $=1 \mathrm{sec}$

Best fit chi squared $=360.256165$

Elapsed time in shore $=12 \mathrm{sec}$

$\begin{array}{llllll}27 & 19.000000 & 0.000000 & 0.000000 & 0.000000 & 0.000000 \\ 28 & -0.014845 & 0.100000 & 0.000000 & 0.000000 & 0.000000 \\ 29 & 0.610310 & 0.000000 & 0.000000 & 0.000000 & 0.000000 \\ 30 & 784.487346 & 0.500000 & -12.725013 & 12.776161 & 12.750587 \\ 31 & 0.002000 & 0.000000 & 0.000000 & 0.000000 & 0.000000 \\ 32 & 0.010000 & 0.000000 & 0.000000 & 0.000000 & 0.000000 \\ 33 & -0.025681 & 0.000000 & 0.000000 & 0.000000 & 0.000000 \\ 34 & 36.845152 & 0.000000 & 0.000000 & 0.000000 & 0.000000 \\ 35 & 0.413371 & 0.005000 & -0.009426 & 0.009558 & 0.009492 \\ 36 & 4311.463344 & 10.000000 & -130.433374 & 137.611303 & 134.022339\end{array}$

data file: $0^{\circ} \mathrm{C}$ _short.dat

impulse file: pos.par

maximum counts 174.000000 at $1.717600 \mathrm{~ns}$ - bin 72

signal counts 90362.000000 over 376 bins

time per bin (ns): 0.858806

Elapsed time in stream $=5 \mathrm{sec}$

Best fit chi squared $=410.176392$

Elapsed time in shore $=30 \mathrm{sec}$

$\begin{array}{llllll}27 & 9.292837 & 0.010000 & 0.000000 & 0.000000 & 0.000000 \\ 28 & 2.352571 & 0.100000 & 0.000000 & 0.000000 & 0.000000 \\ 29 & 0.610310 & 0.000000 & 0.000000 & 0.000000 & 0.000000 \\ 30 & 768.550716 & 50.000000 & -15.880812 & 16.278109 & 16.079460 \\ 31 & 0.002000 & 0.000010 & -0.000132 & 0.000137 & 0.000134 \\ 32 & 0.010000 & 0.000000 & 0.000000 & 0.000000 & 0.000000 \\ 33 & -0.025681 & 0.010000 & -0.001670 & 0.001553 & 0.001611 \\ 34 & 36.845158 & 0.400000 & -2.138347 & 2.286642 & 2.212494 \\ 35 & 0.413371 & 0.000000 & 0.000000 & 0.000000 & 0.000000 \\ 36 & 4311.463344 & 0.000000 & 0.000000 & 0.0(0000 & 0.000000\end{array}$


data file: $-10^{\circ} \mathrm{C}$ _long.dat

impulse file: pos.par

maximum counts 195.000000 at 133.120000$)$ ns - bin 32

signal counts 22799.000000 over 336 bins

time per bin (ns): 16.639799

Elapsed time in stream $=\quad 0 \mathrm{sec}$

Best fit chi squared $=362.528072$

Elapsed time in shore $=8 \mathrm{sec}$

\begin{tabular}{|c|c|c|c|c|}
\hline 6.500000 & 0 secromon & 0 orovyra & o orovgry & $0000 \mathrm{cov}$ \\
\hline 0.168931 & 0.100000 & 0.0000000 & $0.0(0 \times 0)(0)$ & $0.00(X)(X)$ \\
\hline 0.600544 & 0.000000 & $0.000000)$ & 0.0 OYOOOX) & 0.0000000 \\
\hline 919.387172 & 0.500000 & -22.576798 & 22.719832 & 22.648315 \\
\hline 0.002111 & 0.000000 & 0.0000000 & $0.000000)$ & $0.000 \times 00)$ \\
\hline 0.010000 & 0.000000 & 0.0000000 & 0.000000 & $0.00000(X)$ \\
\hline-0.026583 & 0.000000 & 0.000000 & $0.0 \times \times 00000$ & $0.000 \times 000$ \\
\hline 46.635740 & 0.0000000 & 0.0000000 & 0.0 OOOOOKO & 0.0000000 \\
\hline 0.423928 & 0.005000 & -0.012066 & 0.012414 & 0.012240 \\
\hline 6550.731064 & 10.000000 & -379.788259 & 426.529662 & 403.158961 \\
\hline
\end{tabular}

data file: $-10^{\circ} \mathrm{C}_{-}$short.dat

impulse file: pos.par

maximum counts $39.00(0)(0)$ at 1.717600$) \mathrm{ns}-\operatorname{hin} 72$

signal counts 17127.000000 ) over 376 bins

time per bin (ns): 0.858806

Elapsed time in stream $=\quad 0 \mathrm{sec}$

Best fit chi squared $=416.254923$

Elapsed time in shore $=6 \mathrm{sec}$

\begin{tabular}{|c|c|c|c|c|c|}
\hline 27 & 2.262656 & $0.010 \times(00)$ & 0.000 (KX)K) & $0.000(0)(1)$ & $0.00(x)(0)$ \\
\hline & 2.391350 & $0.10 \times(0 \times)$ & 0.0 (O)XOKX) & $0 .(X) O(X)(X)$ & $0.00(x)(K)$ \\
\hline 29 & 0.002111 & $0.00 \times(O X O O)$ & 0.00 OOOKK) & (0.000000) & $0.0(00)(0)$ \\
\hline 30 & $0.010 \times 000$ & $0.000000)$ & $0.00000(0)$ & $0.0(O) O(O) O)$ & $0.00(0)(0)$ \\
\hline 31 & $-0 .(1) 26583$ & $0.010 \%(O)$ & $-0.0 \times(0298$ & $0 .($ OOO)277 & $0.00(1)$ \\
\hline 32 & 46.636057 & $0.400 \times(10)$ & -2.063133 & 2.347277 & 2.20521 \\
\hline 33 & $0.6(0) 544$ & 0.00000000 & $0.0000 \times(X)$ & $0.00000(X)$ & $0.000(0) 0$ \\
\hline 34 & 919.387172 & $0.000(0) 00$ & $0.00000(X)$ & (0.ONOOOOOO & 0.000000 \\
\hline 35 & 0.423928 & $0.00000 \times 0$ & $0.000 \times 0 \times 0)$ & $0.00 \times(x)(x)$ & $0.000(x)$ \\
\hline 36 & 6550.731064 & 0.0000000 & $0.000(0) 00$ & $0.0000(0) 0$ & $0.00(K)$ \\
\hline
\end{tabular}


data file: -20$)^{\circ} \mathrm{C}$ long.dat

impulse file: pos.par

maximum counts $232 .(X X X X X X)$ at $175.95(X) X X)$ ns - hin 36

signal counts $68685.1744(0)$ over 461 bins

time per hin (ns): $1(1.349932$

Elapsed time in stream $=1 \mathrm{sec}$

Best fit chi squared $=50.5 .784818$

Elapsed time in shore $=14 \mathrm{sec}$

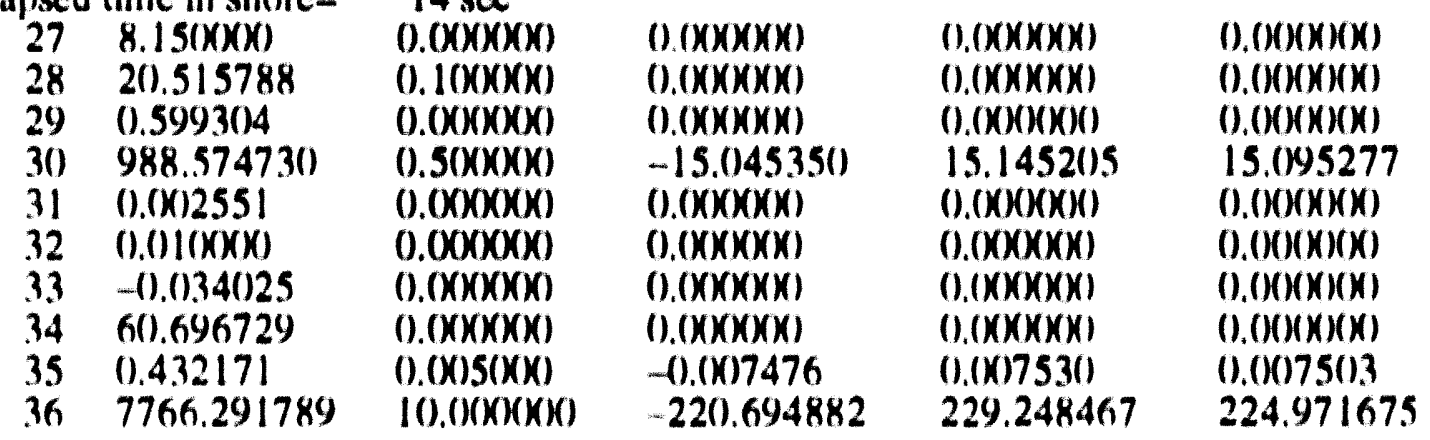

data file: $-200^{\circ} \mathrm{C}_{-}$short dat

impulse file: poss.par

maximum counts 33.(XXXXKX) at $2.57641(x)$ ns -.. hin 73

signal counts $13126 .(X X X)(X X)$ over 376 hins

lime per hin (ns): 0.858806

Elapsed time in stream $=1 \mathrm{sec}$

Best fit chi squared $=349.23(1) 196$

Vilapsed time in shore $=\quad 6 \mathrm{sec}$

\begin{tabular}{|c|c|c|c|c|}
\hline $27 \quad 1.682351$ & $0 .(0) 1(1)(x \times)$ & $0.0 \times(\times \times \times)(1)$ & (). $(x \times x \times)(x)$ & $(1)(0)(x)(x)$ \\
\hline 2.618351 & $0.1(x \times x \times)$ & $0 .(1)(x)(x)(x)$ & $(0.1 \times x \times)(x)$ & ()$.()()(x)(x)$ \\
\hline()$.(x) 2551$ & $0 .(x \times \times \times x)()$ & (1). $(x \times x \times x)(1)$ & $(1.1 \times \times(x)(x)$ & $(0.0)(x)(x)(x)$ \\
\hline$(0.0) 1(x)(0)$ & $0.0 \times x \times x \times x)$ & (). (x) & (). (x) $(x)(x)$ & $0.0(x)(x)(x)$ \\
\hline-1$).(1) 34(1) 26$ & (0.0)1(x)(0) & $-(1,()(x) 3.332$ & (). $(x)(x) 3(1) 2$ & $(0.1)(1)(1317$ \\
\hline $6(0.695421$ & $0.4(x \times)(x)(0)$ & -2.338663 & 2.624686 & 2.481675 \\
\hline (1).5993(1)4 & $0 .(x \times x \times()()$ & (). $(x \times x \times x \times)$ & $(1 .,(x \times x)(x)$ & $(0.1)(x)(x)(x)$ \\
\hline 988.574730 & $0.0 \times(x)(X)(1)$ & (). $(x \times x \times(x)()$ & (). $(x)(x \times(x)$ & 0.0()$(x)(x)$ \\
\hline$(0.432171$ & $0.0 \times(x)(x)(0)$ & $0.000(x)(0)$ & $(0 .(x \times)(x)(x)$ & $(0.0)(x)(x)$ \\
\hline 778 & $(0 .(x)(x)(x)(0)$ & $(0 .(x)(x)()(1)$ & ()$.(x)()(x)()$ & () $.0(x)(x)(x)$ \\
\hline
\end{tabular}


data file: $-300^{\circ} \mathrm{C}_{-}$long.dat

impulse file: pos.par

maximum counts 223.(XXXXXX) at $455.4(X X Y X X) \mathrm{ns}$ - bin 63

signal counts 82433.563200 over 461 hins

lime per bin (ns): 10.349932

Elapsed time in stream = 0 sec

Best fit chi squared= 506.843175

Elapsed time in shore= $\quad 16 \mathrm{sec}$

\begin{tabular}{|c|c|c|c|c|}
\hline & & & & \\
\hline 10.2000000 & $0.0 \times \times \times \times \times \times 0$ & $0.0 \times \times \times \times \times \times)$ & (). $(X \times X \times(X)$ & $0.0(X)(X)(X)$ \\
\hline 20.272622 & $0.1000 \times 00$ & $0.0 \times \times \times \times \times)$ & $0.0 \times \times(X \times X)$ & 0.0()$(x)(X)$ \\
\hline 0.619628 & $0.0 \times 0 \times 0000$ & $0.000 \times \times \times 0$ & $0.0 \times \times \times \times \times(X)$ & $0.00 \times(x)(x)$ \\
\hline $110 \times 1.950739$ & $10.00 \times 0000)$ & -15.064229 & 15.138310 & 15.101269 \\
\hline $0 .(0) 2065$ & $0.0 \times \times \times \times \times 0$ & $0.0 \times \times \times \times \times)$ & $0.0 \times \times \times \times \times x)$ & $0.00(x)(x)$ \\
\hline $0 .(1) 11 \times(\times \times)$ & $0.0 \times \times \times 0 \times 00$ & $0.00 \times 0 \times \times \times)$ & $0.0 \times 0 \times \times \times \times)$ & $0.000 \times 100$ \\
\hline-10.059726 & $0.0 \times \times \times X \times X)$ & (). $(\times \times \times \times \times)$ & $0.0 \times \times \times \times \times X)$ & $0.0(x)(X)(X)$ \\
\hline 125.378777 & $0.0 \times \times \times X \times)$ & $0.0 \times 0 \times \times \times \times)$ & $0 .(\times \times X \times X \times)$ & $0.00(\times)(0 \times)$ \\
\hline 0.43672 & $0.0 \times 050 \times 0$ & $-(0.007142$ & $0.0 \times 107194$ & (0.007168 \\
\hline 966096 & $10 .(6 \times 1 \times \times 0)$ & -243.265036 & $252.8747(13$ & $248.069871)$ \\
\hline 0.0011309 & $0.0 \times \times \times \times \times X)$ & $0 .(X \times X \times \times X X)$ & $0 .(X \times X X X X)$ & $0.00(x)(0 \times)$ \\
\hline 1.806 & $0 .(\times \times \times \times \times X)$ & $0.0 \times \times \times \times \times)$ & $0 .(\times \times \times \times \times X)$ & $0,0(0)(\times)(X)$ \\
\hline
\end{tabular}

data file: $-\mathbf{3 0} 0^{\circ} \mathrm{C}$ short.dal

impulse file: pos.par

maximum counts $56 .(X)(X(X))$ at $1.7176(x) \mathrm{ns}$ - hin 72

signal counts 16557 .(XXXXOX) over 376 bins

time per bin (ns): $0.8588(16$

Elapsed time in stream $=\quad 1 \mathrm{sec}$

Best fit chi squared $=365.09(0827$

Elapsed time in shore $=\quad 49 \mathrm{sec}$

\begin{tabular}{|c|c|c|c|c|}
\hline 2.711669 & $0.011(x \times(x)$ & $0 .(x)(x)(1)(0)$ & (). $1 \times \times \times x \times(x)$ & $0.0(0)(x)(x)$ \\
\hline 2.233180 & $0.16 \times \times \times \times 0)$ & $0 .(x)(x)(x)$ & $0 .(X \times \times) \times(X)$ & $0 .()()(X)(X)$ \\
\hline $0 .(K) 2065$ & $0.0 \times \times \times \times \times x)$ & $\begin{array}{l}(1 .()(X)()() \\
0\end{array}$ & $0 .(X) \times \times \times(X)$ & $0.0(0)(x)(x)$ \\
\hline $0.01(\times \times \times)$ & $0,00 \times 0 \times 0 \times 0$ & $(0 .(x)(x)()()$ & $(0.1 \times 0 \times 0 \times 1)$ & $0.0(x)(x)(x)$ \\
\hline-0.059726 & $0.010 \times 0 \times 0$ & $-(0.0(x) 362$ & $0 .(K \times 0) 331$ & 0.01001 .347 \\
\hline 125.374937 & $0.4(\times \times \times \times \times)$ & -4.047657 & 4.339682 & 4.193670 \\
\hline 0.619628 & $0,(X \times \times \times \times X)$ & $0 .(x)(x)(1)()$ & (). $(X X X \times \times(X)$ & 0.0()$(x)(x)$ \\
\hline $1100.95(0739$ & $0.0 \times \times \times \times \times)$ & $0 .(000(0)(0)$ & (). $(x) \times(x)()$ & 0.0()$(K)(X)$ \\
\hline 0.436724 & $0.00 \times 0 \times \times \times 0$ & $0.0(0)(x)(0)$ & $0 .(x)(x \times(x)$ & 0.0()$(x)(x)$ \\
\hline 8757.966096 & $0.0 \times \times \times 0 \times \times)$ & $0 .(x)(x)(x)$ & $0 .(X X X X \times X)$ & $0.0(1)(K)(X)$ \\
\hline $0 .(x) 1309$ & $0 .(\times \times \times 0 \times 0) 1$ & $-0.0(0) 5.38$ & $0 .(x) 3401) 7$ & $0.0(1) 1972$ \\
\hline $1.806(181$ & $0 .(x) 1(x \times)$ & -0.674928 & 0.983419 & 0.829173 \\
\hline
\end{tabular}




\begin{tabular}{|c|c|c|c|c|c|}
\hline \multirow{2}{*}{\multicolumn{6}{|c|}{ 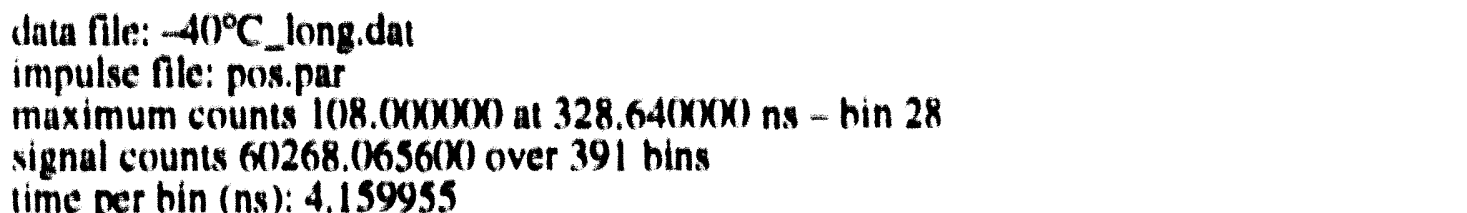 }} \\
\hline & & & & & \\
\hline \multicolumn{2}{|c|}{ Elansed time in stream $=\quad 1 \mathrm{sec}$} & \multicolumn{4}{|c|}{ Hest fit chi squared $=447.449862$} \\
\hline Elapse & time in shore = & $20 \mathrm{sec}$ & & & \\
\hline 27 & $4.5(\times \times \times 1 \times)$ & $0.0 \times 0 \times \times \times 0$ & $0.0 \times(x \times(x) 0$ & $0 .(X)(X) \times()$ & $0.0(X)(X) \times X)$ \\
\hline 28 & 328.307464 & $0.100 \times 100$ & $0 .(\times) \times \times \times x)$ & $0 .(X)(K X \times)$ & $0.0(0) \times(1)$ \\
\hline 29 & 0.641490 & $0.0 \times 100000$ & ().(X)XXKY) & $(0 .(X)(0 \times \times)$ & $0.0(X)(X) \times()$ \\
\hline 30 & 1309.637979 & 10.0000000 & -16.438268 & $16.28941) 7$ & 16.363837 \\
\hline 31 & $0 .(x) 2141$ & $0.0 \times \times \times \times \times \times)$ & $0.0 \times \times O \times \times X)$ & $0.0 \times \times \times \times \times \times)$ & $0.00(x)(x)$ \\
\hline 32 & $(0.011(x \times 0 \times)$ & $0 .(1 \times \times \times 1 \times 00$ & $0.0 \times \times \times \times \times \times)$ & $0.0 \times \times 1 \times(\times) \times)$ & $.0(X X)(X)$ \\
\hline 33 & -0.111787 & $0.0 \times \times \times \times \times x)$ & $0.0 \times \times(x \times X)$ & $0.1 \times \times \times \times \times \times)$ & 0.0 OKKKKX) \\
\hline 34 & 277.8648111 & $0 .(x \times \times) \times(\times)$ & $0,(\times \times \times \times \times \times)$ & $0 .(\times \times \times \times \times \times)$ & $0.0 \times(X) \times()$ \\
\hline 35 & 0.466828 & $0.0 \times 1 \times 0 \times 0$ & -0.006477 & $0.0 \times 105(14$ & $0.0 \times 16490$ \\
\hline 36 & 11290.692387 & $10.00 \times(\times) \times 10)$ & $-6.36 .43(1454$ & 698.292553 & 667.3615013 \\
\hline 37 & $(0 .(x) 1.328$ & $0.0 \times X \times X \times X)$ & $0 .(\times \times O \times \times \times)$ & $0.0 \times \times \times \times \times X)$ & $0.0 \times 1 \times \times \times)$ \\
\hline 38 & & $0 .(X \times X \times \times \times)$ & ().(XXXXXY) & $(0,(X \times X \times \times \times)$ & $0 .(X)(X \times X)$ \\
\hline
\end{tabular}

data lile: $-400^{\circ} \mathrm{C}_{-}$shurt dat

impulse file: pos.par

maximum counts $44 .(0 X X X X)$ at $1.7176 \times(X) \mathrm{ns}$ - hin 72

signal counts $10237 .(O X Y(X)(X)$ over 376 hins

time per hin (ns): 0.858806

Eilapsed time in stream $=1$ sec

Best fil chi squared $=421.7074015$

Elapsed time in shore $=\quad \mathbf{3 4} \mathrm{sec}$

\begin{tabular}{|c|c|c|c|c|}
\hline 2.168182 & $0 .(1)(x \times \times)$ & $0.0 \times \times \times \times \times x)$ & $0(x \times \times \times \times \times)$ & $0.000(0) 0 \times$ \\
\hline 2271330 & (1) $10 \times(x \times(x)$ & (1). $(x \times(x) \times \times)$ & $0.0 \times x \times x \times x)$ & $(0.0()(x)(x)$ \\
\hline$(0.0 \times 12141$ & $0.0 \times \times \times \times \times \times)$ & (). $(x \times X \times X X)$ & $0 .(x)(x \times x \times)$ & $0.0(0)(x)(1)(1)$ \\
\hline$(0.0) 1(x \times \times)$ & $0.0 \times(X \times X \times X)$ & (). $(x \times x \times x \times)$ & $(0).(X X X \times(X))$ & $(0.0)(1)(x)(x)$ \\
\hline$-(0.111787$ & $0 .(x \times())](x)$ & $-(1.00 \times 1461$ & $0.0 \times \times) 416$ & $0.00(14.39$ \\
\hline 277.855195 & $0.4(\times \times \times) \times)$ & -7.629966 & 8.06628 .3 & 7.848125 \\
\hline $0.64149(1)$ & $(1) .(X \times X \times(x))$ & (). $(X \times X X X X)$ & $(0.1 \times(x)(x)(1)$ & $(0.0()(x)(x)$ \\
\hline 1.309 .637979 & $(0.1 \times \times(x \times) \times)$ & $0 .(x \times \times \times \times x)$ & $0.0(x)(x)(1)$ & $(0.0)(x)(x)$ \\
\hline 0.466828 & $0.0 \times \times \times \times \times)$ & $0.0 \times 100(1)$ & $0.0(x)(x)(1)$ & 0.0()$(x)(x)$ \\
\hline 11290.692387 & $0 .(x)(x)(x)$ & $0.0(1)(X 00)$ & $0.0(x)(00)(0)$ & $0.00(x)(x)$ \\
\hline 0.0011328 & $(0 .(x)(x) 1(x)$ & $-0.0(\times)+47$ & $(0.1 \times 10506$ & $0.0(0)(1476$ \\
\hline 3.2344 .34 & $(0.01(x)(0)$ & -1.424 .384 & 1.776422 & $1.6(1)(14(1) ?$ \\
\hline
\end{tabular}


data file: $-50^{\circ} \mathrm{C}$ - long.dat

impulse file: pos.par

maximum counts $180 .(X X X X X X)$ at $20.7(X X X X X)$ ns - hin 25

signal counts $111562.2160 \times 00$ over 465 hins

lime per bin (ns): 10.349933

Elapsed time in sucam $=1 \mathrm{sec}$

Best fit chi squared $=49(0.098430$

Elapsed time in shore= $64 \mathrm{sec}$

\begin{tabular}{|c|c|c|c|c|}
\hline 16 anowy & & & & \\
\hline $16.4(x)(0)$ & $0.0 \times 0 \times 0 \times \times 0$ & $0.0 \times \times \times \times \times \times)$ & $0 .(X \times 1 \times \times \times)$ & $0.00(0 \times(x) \times 0$ \\
\hline $20.418+01$ & $0.1(X \times X \times X)$ & $0.0 \times(\times \times \times)(0)$ & (). $(X \times X \times(X X)$ & $0 .(X) \times()(X)$ \\
\hline 0.791192 & $0.00 \times 0 \times 0 \times 0$ & $0.0 \times 1 \times \times \times \times)$ & $0.0 \times(x)(x)(0)$ & $0.0(0)(x)(X)$ \\
\hline 1771.85869 & $10.0 \times 0 \times(\times) \times$ & -64.213826 & 61.385539 & 62.799683 \\
\hline $0 .(x) 2.494$ & $0.0 \times(\times) \times X)$ & $0 .(x) \times X \times(X)$ & $0.0 \times \times \times \times \times())$ & $0.0(x)(x)(X)$ \\
\hline 4.949857 & $0.0 \times \times \times \times \times 0)$ & $0.0 \times 1 \times \times \times \times)$ & $0 .(1 \times 0 \times \times \times)$ & $0.00(X) \times(X)$ \\
\hline$\approx 164663$ & $0.0 \times x) 1(x)$ & 0.024146 & 0.0119169 & 0.021657 \\
\hline $46(1,248257$ & $10 .(x \times x \times x \times X)$ & $-33.6(14481$ & $37.3591(14$ & 35.481793 \\
\hline 0.367561 & $0.00500 \times 0$ & -0.012584 & 0.012993 & 0.012789 \\
\hline 11721.254495 & 10.00000000 & $-6(12.946543$ & $660.9946611)$ & 631.9706012 \\
\hline$(0.6 \times 12916$ & (). $(X \times X \times X \times X)$ & (). $(X X X X \times X X)$ & (). $(x \times x \times x \times)$ & $0 .(0)(x)(x)$ \\
\hline $0.01(x \times \times)$ & $0 .(X \times X \times 1 \times X)$ & $0 .(\times \times X \times \times)$ & (). $(x \times X \times \times \times X)$ & $0.0(1)(x)(x)$ \\
\hline
\end{tabular}

data lile: $=50{ }^{\circ} \mathrm{C}_{\text {s. }}$ short.dat

impulse file: pos.par

maximum counts $82 .(X X X X X X)$ at $2.5764(X) \mathrm{ns}-$ hin 73

signal counts 124() 5 . ( $X X X X X X)$ over 376 hins

lime per hin (ns): 0.858806

Elapsed time in stream $=8 \mathrm{sec}$

Best fit chi squared= $370.5(\times) 585$

Elapsed time in shore $=97 \mathrm{sec}$

\begin{tabular}{|c|c|c|c|c|}
\hline 3.258610 & $(0.01(x \times x)$ & $0 .(x \times \times \times \times \times)$ & $0 .(x \times x \times x)$ & $0.0(x)(x)(x)$ \\
\hline 2.746147 & (1) $10 \times \times \times \times \times)$ & $0 .(x \times x \times x \times)$ & (1). 1 (x)XXXX) & $(0 .()(x)(x)(x)$ \\
\hline$(0 .(x), 3() 37$ & $0 .(x \times \times \times \times \times)$ & (). (XXXXXXX) & (1). (XXXXXX) & ()$,(K)(K)(X)$ \\
\hline 4.982791 & $(1)(x)](x \times x)$ & $=1.465531)$ & 1.4655311 & 1.4655311 \\
\hline (1). 164741 & $0 .(x \times)](x)$ & (1).0(x) 570 & $(1)(x \times 1) 521$ & $0.0(1)(1545$ \\
\hline $47(1) .1824(19$ & $0.4(x \times x \times x)$ & -10.858670 & $11.4(13679$ & 11.131174 \\
\hline 0.791192 & $0.0 x(x)(x)(x)$ & $0,0 x \times(x) \times(x)$ & (). (X)XXKX) & $0.0(0)(x)(x)$ \\
\hline 1771.085725 & $0 .(x)(x)()()$ & $0 .(x)(x) \times x)$ & (1). $(x \times x \times x \times x)$ & $0.0(0)(x)(x)$ \\
\hline 0.367561 & $0 .(x \times \times)(x \times)$ & $0.0 \times \times \times \times \times()$ & (1). $(x \times X \times X \times(x)$ & 0.0()$(x)(x)$ \\
\hline 11721.254495 & $0.0(0)(x)(0)$ & $0 .(x)(x)(x)$ & $0 .(x)(x)(x)()$ & $0.0(0)(x)(x)$ \\
\hline (). (1) 2951 & $0.0 \times \times(x) 10$ & $-1) .(x \times 1) 429$ & $0 .(x \times 1) 429$ & $0.0(1) 429$ \\
\hline $0.01100(0)$ & $0.0 \times(x \times \times \times \times)$ & $0 .(x) \times \times \times \times()$ & $(0.0 \times \times \times \times X)$ & $(0.0)(x)(x)$ \\
\hline
\end{tabular}


data file: $-60^{\circ} \mathrm{C}$ _long.dat

impulse file: pos.par

maximum counts $179.0 \%(X X X)$ at $20.7(X X X X)$ ns - hin 25

signal counts $75(145.38240(0)$ over 465 hins

lime per bin (ns): 10.349933

Elapsed time in stream $=4 \mathrm{sec}$

Best fit chi squared $=467.825155$

Elapsed time in shore $=258 \mathrm{sec}$

\begin{tabular}{|c|c|c|c|c|}
\hline $27 \quad 10.9()(x)(x)$ & $0.0 \times \times \times \times \times 0)$ & $0 .(X \times X \times(X \times)$ & $0.0 \times \times \times(\times) \times 0)$ & $0.10)(\times 0)(\times 0)$ \\
\hline 20.574852 & $0.1000 \times 0$ & $0.0 \times \times \times \times \times)$ & (1). $1 \times \times \times \times \times \times)$ & $0.00(x)(x)$ \\
\hline 0.678770 & $0.0 \times \times(X) \times X)$ & $0.0 \times x \times(x)$ & $0 .(X \times X \times \times \times X)$ & (). $(x)(x)(X)$ \\
\hline $3(145.3237(1)$ & $100.0000 \times 1 \times 0)$ & -317.434545 & 277.636238 & 297.535392 \\
\hline $0 .(x) 4119$ & $0.0 \times \times \times O \times \times 1$ & $0.0 \times \times \times \times \times()$ & $0.1 \times \times \times \times(X)$ & $0.0(0)(x)(X)$ \\
\hline 7.938293 & $0.0 \times \times \times \times \times)$ & $0.0 \times 0 \times(\times \times)$ & $0 .(1 \times \times \times 1 \times)$ & $(0.0()(x)(x)$ \\
\hline-10.129422 & $0 .(0) 1(\times \times \times)$ & -0.062202 & 0.032391 & $0 .(147296$ \\
\hline 88.471552 & $1(1)(1)(1)(x)(x)(1)$ & -126.7830158 & 167.8859 .39 & 147.334499 \\
\hline 0.442946 & $0.05(X) \times()$ & -0.043846 & 0.1146412 & (1).045129 \\
\hline $1,3,39,3,49957()$ & $500.0(\times 0 \times \times) 0$ & -1314.024926 & $1696.43,3699$ & 1505.229313 \\
\hline$(0 .(x) 3587$ & $0.0 \times \times \times \times \times \times 0$ & $(\because x) \times x \times x)$ & $(0 .(X \times X \times X \times)$ & $0.0(x)(x)(x)$ \\
\hline$(1),(1)(x \times X)$ & (). $(x \times \times \times \times x)$ & (). (XXXXXX) & (1). $(X X X X \times X X)$ & $0.0(0)(x) \times()$ \\
\hline
\end{tabular}

data fille: $-611^{\circ} \mathrm{C}_{\text {short dat }}$

impulse filc: pos.par

inaximum counts $58.0 \times(x)(X X)$ at $2.5764(x)$ ns - hin 7.3

signal counts $6184 .(X \times X \times) \times$ over 376 hins

lime per hin (ns): $0.8588(16$

Elapsed time in stream $=2 \mathrm{sec}$

Best fit chi squared= 391.213250

Blapsed time in shore $=75 \mathrm{sec}$

\begin{tabular}{|c|c|c|c|c|}
\hline $27 \quad 2.684620$ & $0 .(1) 1(x)(x)$ & $0 .(x \times x \times x)$ & $0.6 \times \times(x) \times(x)$ & $(0.1)(0)(x)(x)$ \\
\hline 2.551928 & $0.1(\times \times \times \times \times)$ & $0 .(X)(x)(1)$ & $0.1 \times \times \times \times \times \times)$ & $\left(1.0^{\prime \prime}\right)(x)(x)$ \\
\hline$(1 .(x) 4119$ & $0 .(X X X X \times X X)$ & ().(X)(X)XX)(1) & $(0,(x \times x \times x)$ & $(0.0)(1)(x)(x)$ \\
\hline $7.938(12.3$ & $0.05(x \times x)$ & -1.879945 & 1.87994 .5 & 1.8799 .45 \\
\hline .0 .129422 & $0 .(x \times)(x)(x)$ & $0 .(x \times X \times \times X)$ & $0.1 \times \times x \times x)$ & $(0.1)(1)(X)(X)$ \\
\hline 885.471552 & (1). $(x \times x \times(x)(1)$ & $0.0(x) \times(x)(x)$ & (1). $(x \times x \times x \times)$ & $(0 .()(1)(x)(x)$ \\
\hline (1.678770) & (). (XKXXXX) & $0 .(x \times x \times x \times()$ & (). $(x \times x \times x)$ & $(0.0)(x)(x)(x)$ \\
\hline $3(145.3237(1) 7$ & (). (x)XX(x)1) & $0 .(x \times x \times x \times()$ & (1). $(x \times x \times x \times x)$ & $(0.0)(x)(x)(x)$ \\
\hline 0.442946 & $0 .(x) \times(x) \times x)$ & $0 .(x \times x \times x \times x)$ & $11(X) \times x \times(X)$ & $(0,0)()(x)(x)$ \\
\hline 13393.49957() & $0 .(x)(x)()()$ & $0 .(x)(x)(10)$ & $0 .(x)()(x) 0$ & $(1)(1)(x)(x)$ \\
\hline (1).(1)(13587 & $0 .(x)(010)$ & $-10.0(0) 415$ & $0.1 \times 1) 4(144$ & $0.0(10) 411)$ \\
\hline$(1)(1) 1(1)(x)$ & $0 .(x)(x)()()$ & $0 .(x)(x)()(1)$ & (1) (x)(x)(x)(x) & $0.0(0)(x)(x)$ \\
\hline
\end{tabular}


data file: $-80^{\circ} \mathrm{C} \_$long.dat

impulse file: pos.par

maximum counts 176.000000 at 20.700000 ns - bin 25

signal counts 52162.406400 over 465 bins

time per bin (ns): 10.349933

Elapsed time in stream $=1 \mathrm{sec}$

Best fit chi squared $=465.352954$

Elapsed time in shore $=396 \mathrm{sec}$

$\begin{array}{llllll}27 & 10.300000 & 0.000000 & 0.000000 & 0.000000 & 0.000000 \\ 28 & 20.813951 & 0.100000 & 0.000000 & 0.000000 & 0.000000 \\ 29 & 0.012998 & 0.000000 & 0.000000 & 0.000000 & 0.000000 \\ 30 & 0.010000 & 0.000000 & 0.000000 & 0.000000 & 0.000000 \\ 31 & 0.034645 & 0.000100 & -0.003576 & 0.003797 & 0.003686 \\ 32 & 84.395609 & 0.100000 & -12.586945 & 14.404769 & 13.495857 \\ 33 & 0.170706 & 0.005000 & -0.020667 & 0.022546 & 0.021607 \\ 34 & 2218.086898 & 10.000000 & -291.147340 & 330.360832 & 310.754086 \\ 35 & 0.781651 & 0.005000 & -0.022840 & 0.020808 & 0.021824 \\ 36 & 17980.022978 & 100.000000 & -1297.300605 & 1573.004644 & 1435.152624\end{array}$

data file: $-80^{\circ} \mathrm{C} \_$short.dat

impulse file: pos.par

maximum counts 46.000000 at $1.717600 \mathrm{~ns}$ - bin 72

signal counts 3348.000000 over 376 bins

time per bin (ns): 0.858806

Elapsed time in stream $=0 \mathrm{sec}$

Best fit chi squared $=379.014768$

Elapsed time in shore $=6 \mathrm{sec}$

$\begin{array}{rlllll}27 & 1.671107 & 0.010000 & 0.000000 & 0.000000 & 0.000000 \\ 28 & 2.811952 & 0.100000 & 0.000000 & 0.000000 & 0.000000 \\ 29 & 0.035770 & 0.000000 & 0.000000 & 0.000000 & 0.000000 \\ 30 & 39.482081 & 1.500000 & -5.006340 & 5.610159 & 5.308250 \\ 31 & 0.170706 & 0.000000 & 0.000000 & 0.000000 & 0.000000 \\ 32 & 2218.086898 & 0.000000 & 0.000000 & 0.000000 & 0.000000 \\ 33 & 0.781651 & 0.000000 & 0.000000 & 0.000000 & 0.000000 \\ 34 & 17980.022978 & 0.000000 & 0.000000 & 0.000000 & 0.000000 \\ 35 & 0.011873 & 0.001000 & -0.001119 & 0.001194 & 0.001156 \\ 36 & 0.010000 & 0.000000 & 0.000000 & 0.000000 & 0.000000\end{array}$


data file: $-100^{\circ} \mathrm{C} \_$long.dat

impulse file: pos.par

maximum counts 272.000000 at $20.700000 \mathrm{~ns}$ - bin 22

signal counts 29380.940800 over 462 bins

time per bin (ns): 10.349932

Elapsed time in stream $=8 \mathrm{sec}$

Best fit chi squared $=475.250111$

Elapsed time in shore $=22 \mathrm{sec}$

$\begin{array}{llllll}27 & 6.359429 & 0.010000 & 0.000000 & 0.000000 & 0.000000 \\ 28 & 20.746489 & 0.100000 & 0.000000 & 0.000000 & 0.000000 \\ 29 & 0.171484 & 0.000000 & 0.000000 & 0.000000 & 0.000000 \\ 30 & 49.926571 & 1.000000 & 0.000000 & 0.000000 & 0.000000 \\ 31 & 0.306027 & 0.001000 & 0.000000 & 0.000000 & 0.000000 \\ 32 & 385.598251 & 10.000000 & 0.000000 & 0.000000 & 0.000000 \\ 33 & 0.480394 & 0.005000 & -0.025565 & 0.024105 & 0.024835 \\ 34 & 3523.841313 & 10.000000 & -364.878746 & 413.725795 & 389.302270 \\ 35 & 0.042094 & 0.000000 & 0.000000 & 0.000000 & 0.000000 \\ 36 & 0.010000 & 0.000000 & 0.000000 & 0.000000 & 0.000000\end{array}$

data file: $-100^{\circ} \mathrm{C} \_$short.dai

impulse file: pos.par

maximum counts 94.000000 at $2.576400 \mathrm{~ns}-\operatorname{bin} 73$

signal counts 4319.000000 over 376 bins

time per bin (ns): 0.858806

Elapsed time in stream $=2 \mathrm{sec}$

Best fit chi squared $=395.984016$

Elapsed time in shore $=31 \mathrm{sec}$

$\begin{array}{llllll}27 & 1.567661 & 0.010000 & 0.000000 & 0.000000 & 0.000000 \\ 28 & 2.479115 & 0.100000 & 0.000000 & 0.000000 & 0.000000 \\ 29 & 0.042109 & 0.000000 & 0.000000 & 0.000000 & 0.000000 \\ 30 & 0.010000 & 0.000000 & 0.000000 & 0.000000 & 0.000000 \\ 31 & 0.101060 & 0.010000 & -0.013489 & 0.014757 & 0.014123 \\ 32 & 19.816957 & 0.400000 & -3.298995 & 3.866941 & 3.582968 \\ 33 & 0.376436 & 0.010000 & -0.015091 & 0.013689 & 0.014390 \\ 34 & 170.955402 & 10.000000 & -15.956594 & 18.002209 & 16.979401 \\ 35 & 0.480394 & 0.000000 & 0.000000 & 0.000000 & 0.000000 \\ 36 & 3523.841313 & 0.000000 & 0.000000 & 0.000000 & 0.000000\end{array}$


Table D.1: Calculated reduced $\chi^{2}$ values for temporal luminescence intensity least squares fits.

\begin{tabular}{|c|c|}
\hline File & Reduced $\chi^{2}$ \\
\hline 50_long & 1.133 \\
50_short & 1.420 \\
40_long & 1.110 \\
40 _short & 0.9688 \\
$30^{\circ} \mathrm{C}$ _long & 0.9319 \\
$30^{\circ} \mathrm{C}$ _short & 0.8850 \\
$23^{\circ} \mathrm{C}$ _Bicron_long & 1.028 \\
$23^{\circ} \mathrm{C}$ _Bicron_short & 1.114 \\
$23^{\circ} \mathrm{C}$ _Horiba_long & 1.160 \\
$23^{\circ} \mathrm{C}$ _Horiba_short & 1.089 \\
$23^{\circ} \mathrm{C}$ _Rexon_long & 1.119 \\
$23^{\circ} \mathrm{C}$ _Rexon_short & 1.053 \\
$23^{\circ} \mathrm{C}$ _long & 1.178 \\
$23^{\circ} \mathrm{C}$ _short & 1.095 \\
20 & 1.150 \\
$20^{\circ} \mathrm{C}$ _long & 1.066 \\
$10^{\circ}$ _short & 0.9840 \\
$10^{\circ} \mathrm{C}$ _short & 1.018 \\
$0^{\circ} \mathrm{C}$ _Bicron_long & 1.026 \\
$0^{\circ} \mathrm{C}$ _Bicron_short & 1.034 \\
$0^{\circ} \mathrm{C}$ _Horiba_long & 1.079 \\
$0^{\circ} \mathrm{C}$ _Horiba_short & 1.081 \\
\hline
\end{tabular}

\begin{tabular}{|c|c|}
\hline File & Reduced $\chi^{2}$ \\
\hline $0^{\circ} \mathrm{C}$ _Rexon_long & 1.163 \\
$0^{\circ} \mathrm{C}$ _Rexon_short & 0.9978 \\
$0^{\circ} \mathrm{C}$ _long & 1.088 \\
$0^{\circ} \mathrm{C}$ _short & 1.112 \\
$-10^{\circ} \mathrm{C}$ _long & 1.095 \\
$-10^{\circ} \mathrm{C}$ _short & 1.122 \\
$-20^{\circ} \mathrm{C}$ _long & 1.109 \\
$-20^{\circ} \mathrm{C}$ _short & 0.9413 \\
$-30^{\circ} \mathrm{C}$ _long & 1.111 \\
$-30^{\circ} \mathrm{C}$ _short & 0.9894 \\
$-40^{\circ} \mathrm{C}$ _long & 1.159 \\
$-40^{\circ} \mathrm{C}$ _short & 1.143 \\
$-50^{\circ} \mathrm{C}$ _long & 1.070 \\
$-50^{\circ} \mathrm{C}$ _short & 1.004 \\
$-60^{\circ} \mathrm{C}$ _long & 1.021 \\
$-60^{\circ} \mathrm{C}$ _short & 1.054 \\
$-80^{\circ} \mathrm{C}$ _long & 1.018 \\
$-80^{\circ} \mathrm{C}$ _short & 1.022 \\
$-100^{\circ} \mathrm{C}$ _long & 1.047 \\
$-100^{\circ} \mathrm{C}$ sshort & 1.073 \\
\hline
\end{tabular}




\section{REFERENCES}




\section{REFERENCES}

[ALA86] J. Alarja, et al., "Charged Particles Identification with a CsI(Tl) Scintillator," Nucl. Instr. and Meth., vol. A242, pp. 352-354, 1986.

[ALU86] N. L. Aluker, V. V. Gavrilov, and R. G. Deich, "Energy Transfer by Luminescence Centers in Alkali-Halide Crystallophosphors," Izv. Akad. Nauk SSSR Fiz., vol. 50, no. 3, pp. 105-108, 1986.

[ALU88] E. D. Aluker, V. V. Gavrilov, R. G. Deich, and S. A. Chernov, "Ultrafast Luminescence of CsI," JETP Lett., vol. 47, no. 2, pp. 142-144, 1988.

[AVS90] B. S. Avset, et al., "Silicon Drift Photodiodes," Nucl. Instr. and Meth., vol. A288, pp. 131-136, 1990.

[BAT69] J. E. Bateman, "Some New Scintillator-Photodiode Detectors for High Energy Charged Particles," Nucl. Instr. and Meth., vol. 67, pp. 93-102, 1969.

[BEN89] F. Benrachi, et al., "Investigation of the Performance of CsI(Tl) for Charged Particle Identification by Pulse-Shape Analysis," Nucl. Instr. and Meth., vol. A281, pp. 137-142, 1989.

[BIR64] J. B. Birks, The Theory and Practice of Scintillation Counting, Pergamon Press, Oxford, 1964.

[BIR91] A. J. Bird, A. J. Dean, K. J. Grant, G. Malaguti, S. D. Mullerworth, and B. M. Swinyard, "Design Characteristics of a Low-energy Gamma-ray Imager for Future Space Missions," Nucl. Instr. and Meth., vol. A310, pp. 323-326, 1991 .

[BIR92] A. J. Bird, T. Carter, A. J. Dean, D. Ramsden, and B. M. Swinyard, "The Optimization of Small CsI(Tl) Gamma-ray Detectors," presented at the 1992 IEEE Nuclear Science Symposium and to appear in the Conference Record.

[BLU85] E. Blucher, et al., "Progress on the CLEO II CsI Electromagnetic Calorimeter," IEEE Trans. Nucl. Sci., vol. 32, no. 1, pp. 716-719, 1985.

[BLU86] E. Blucher, et al., "Tests of Cesium Iodide Crystals for an Electromagnetic Calorimeter," Nucl. Instr. and Meth., vol. A249, pp. 201-227, 1986.

[BOL61] L. M. Bollinger and G. E. Thomas, "Measurement of the Time Dependence of Scintillation Intensity by a Delayed-Coincidence Method," Rev. Sci. Instr., vol. 32, pp. 1044-1050, 1961 .

[CHR76] O. Christensen, "Quantum Efficiency of the Internal Photoelectric Effect in Silicon and Germanium," J. Appl. Phys., vol. 47, pp. 689-695, 1976.

[CIA68] M. Ciampi, L. Daddi, and V. D'Angelo, "Fitting of Gaussians to Peaks by a Maximum Probability Method," Nucl. Instr. and Meth., vol. 66, pp. 102-104, 1968. 
[CIA70] M. Ciampi and L. Daddi, "Statistical Error in the Determination of Peak Parameters as a Function of Channel Width," Int. J. Appl. Rad. and Isotopes, vol. 21 , pp. 47-49, 1970.

[CRA74] C. J. Crannell, R. J. Kurz, and W. Viehmann, "Characteristics of Cesium Iodide for Use as a Particle Discriminator for High-Energy Cosmic Rays," Nucl. Instr. and Meth., vol. 115, pp. 253-261, 1974.

[DEA87] A. J. Dean, G. Graham, C. J. Hopkins, D. Ramsden, and M. Lei, "A Position Sensitive Gamma-ray Detector which Employs Photodiode and CsI(TI) Crystals," IEEE Trans. Nucl. Sci., vol. 34, no. 4, pp. 1089-1092, 1987.

[DEI89] R. Deich, M. Karklina, and L. Nagli, "Intraband Luminescence of CsI Crystal," Solid State Comm., vol. 71, no. 10, pp. 859-862, 1989.

[DE192] R. G. Deich and M. S. Abdrakhmanov, "Intraband Luminescence in Ionic Crystals," Phys. Status Solidi B, vol. 171, pp. 263-273, 1992.

[DER90] S. E. Derenzo, W. W. Moses, J. L. C'ahoon, R. C. C. Perera, and J. E. Litton, "Prospects for New Inorganic Scintillators," IEEE Trans. Nucl. Sci., vol. 37, pp. 203-208, 1990.

[DER92] S. E. Derenzo, W. W. Moses, J. L. Cahoon, T. A. DeVol, and L. Boatner, "X-ray Fluorescence Measurements of 412 Inorganic Compounds," Conference Record of 1991 IEEE Nuclear Science Symposium, vol. 1, pp. 143-147, 1991. Also submitted to IEEE Trans. Nucl. Sci.

[DER93] S. E. Derenzo, "TAUFIT- a fitting program to determine fluorescent decay components as sum of exponentials convolved with an impulse response," Lawrence Berkeley Laboratory Report No. LBL-33050, 1993.

[DIE72] H. B. Dietrich and R. B. Murray, "Kinetics of the Diffusion of Self-Trapped Holes in Alkali Halide Scintillators," J. Lumin., vol. 5, pp. 155-170, 1972.

[DON68] J. M. Donahue and K. Teegarden, "Luminescence from Perturbed Exciton States," J. Phys. Chem. Solids, vol. 29, pp. 2141-2151, 1968.

[FAL84] W. R. Falk, "Data Reduction from Experimental Histograms," Nucl. Instr. and Meth., vol. 220, pp. 473-478, 1984.

[FAN64] C. Y. Fan, "Detection of Scintillation Photons with Photodiodes," Rev. Sci. Instr., vol. 35, no. 2, pp. 158-163, 1964.

[FAR82] M. R. Farukhi, "Recent Developments in Scintillation Detectors for X-ray CT and Positron CT Applications," IEEE Trans. Nucl. Sci., vol. 29, no. 3, pp. 1237-1249, June 1982.

[FON68] M. P. Fontana and W. J. Van Sciver, "Energy Transfer and Optical Properties of $\mathrm{Tl}^{+}$Centers in NaI(Tl) Crystals," Phys. Rev., vol. 168, no. 3, pp. 960-964, 1968.

[GEI82] J. Geist, W. K. Gladden, and E. F. Zalewski, "Physics of Photon-flux Measurements with Silicon Photodiodes," J. Opt. Soc. Am., vol. 72, no. 8, pp. 1068-1075, 1982. 
[GON88] W. G. Gong, et al., "Resolution Tests of CsI(T1) Scintillators Read Out by PIN Diodes," Nucl. Instr. and Meth., vol. A268, pp. 190-199, 1988.

[GRA84] B. C. Grabmaier, "Crystal Scintillators," IEEE Trans. Nucl. Sci., vol. 31, no. 1, pp. 372-376, 1984.

[GRA85] H. Grassmann, E. Lorenz, and H. G. Moser, "Properties of CsI(Tl) Renaissance of an Old Scintillation Material," Nucl. Instr. and Meth., vol. 228, pp. 323-326, 1985.

[GUT74] V. B. Gutan, L. M. Shamovskii, A. A. Dunina, and B. S. Gorobets, "Two Types of Luminescence Centers in CsI(Tl)," Opt. Spectrosc., vol. 37, no. 4, pp. 407-410, 1974.

[GWI63] R. Gwin and R. B. Murray, "Scintillation Process in CsI(Tl). II. Emission Spectra and the Possible Role of Self-Trapped Holes," Phys. Rev., vol. 131, no. 2, pp. 508-512, 1963.

[HEA84] M. A. Heald, "On Choosing the Bin Width of a Gaussian Histogram," Am. J. Phys., vol. 52, no. 3, pp. 254-255, 1984.

[HEA79] R. L. Heath, R. Hofstadter, and E. B. Hughes, "Inorganic Scintillators, A Review of Techniques and Applications," Nucl. Instr. and Meth., vol. 162, pp. $431-476,1979$.

[HOF50] R. Hofstadter, "Properties of Scintillation Materials," Nucleonics, vol. 6, no. 5 , pp. 70-73, 1950.

[HOL88] I. Holl, E. Lorenz, and G. Mageras, "A Measurement of the Light Yield of Common Inorganic Scintillators," IEEE Trans. Nucl. Sci., vol. 35, no. 1, pp. 105-109, 1988.

[HRE60] G. Hrehuss, "A New Method of Mass Discrimination," Nucl. Instr. and Meth., vol. 8, pp. 344-347, 1960.

[HUT73] D. A. Hutcheon, "The Effect of Channel Width in Determining Centroid Shifts," Nucl. Instr. and Meth., vol. 113, pp. 221-223, 1973.

[JAM92] K. M. James, M. J. Masterson, and R. Farrell, "Performance Evaluation of New Large-Area Avalanche Photodiodes for Scintillation Spectroscopy," Nucl. Instr. and Meth., vol. A313, pp. 196-202, 1992.

[JIN92] T. Jing, et al., "Enhanced Columnar Structure in CsI Layer by Substrate Patterning," IEEE Trans. Nucl. Sci., vol. 39, no. 5, pp. 1195-1198, 1992.

[JOH53] P. D. Johnson and F. E. Williams, "Electron Traps in the Thallium-Activated Potassium Chloride Phosphor," J. Chem. Phys., vol. 21, no. 1, pp. 125-130, 1953.

[JON60] D. A. Jones and A. Ward, "The Efficiency and Main Decay Time of Csl(Tl) for Electrons and Alpha Particles in Crystals with Different Thallium Concentrations," Proc. Phys. Soc., vol. 75, pp. 931-933, 1960. 
[KAU70] R. G. Kaufman, W. B. Hadley, and H. N. Hersh, "The Scintillation Mechanism in Thallium Doped Alkali Halides," IEEE Trans. Nucl. Sci., vol. 17, no. 3, pp. 82-88, 1970 .

[KEI68] G. Keil, "Gamma-ray Spectrometry with a Scintillator - Photodiode Combination," Nucl. Instr. and Meth., vol. 66, pp. 167-172, 1968.

[KIL90] U. Kilgus, R. Kotthaus, and E. Lange, "Prospects of CsI(Tl) - Photodiode Detectors for Low-Level Spectroscopy," Nucl. Instr. and Meth., vol. A297, pp. 425-440, 1990.

[KNO88] G. F. Knoll, T. F. Knoll, and T. M. Henderson, "Light Collection in Scintillation Detector Composites for Neutron Detection," IEEE Trans. Nucl. Sci., vol. 35, no. 1, pp. 872-875, 1988.

[KNO89] G. F. Knoll, Radiation Detection and Measurement, 2nd ed., John Wiley \& Sons, New York, 1989.

[KOB89] M. Kobayashi, P. Carlson, and S. Berglund, "Temperature Dependence of CsI(Tl) Scintillation Yield for Cosmic Muons, 5 and 1.25 MeV Gamma Rays," Nucl. Instr. and Meth., vol. A281, pp. 192-196, 1989.

[KRE87] P. Kreutz, A. Kuhmichel, C. Pinkenburg, and J. Pochodzalla, "Photodiode Readout and Pulse Shape Analysis of CSI(TI) Scintillator Signals," Nucl. Instr. and Meth., vol. A260, pp. 12()-123, 1987.

[LAG61] R. G. Lagu and B. V. Thosar, "Fluorescence and Scintillation Spectra of CsI(Tl) Crystals," Proc. Ind. Acad. Sci., vol. 53A, pp. 219-226, 1961.

[LAV83] M. Laval, et al., "Barium Fluoride - Inorganic Scintillator for Subnanosecond Timing," Nucl. Instr. and Meth., vol. 206, pp. 169-176, 1983.

[MAS66] S. Masunaga, I. Morita, and M. Ishiguro, "Optical Properties of CsI(Tl) and CsBr(Tl)," J. Phys. Soc. Japan, vol. 21, no. 4, pp. 638-644, 1966.

[MEI88] R. J. Meijer, et al., "A Compact Multidetector System for Highly Energetic Charged Particles," Nucl. Instr. and Meth., vol. A264, pp. 285-290, 1988.

[MOR59] Z. L. Morgenshtern, "Luminescence in Unactivated CsI Monocrystals," Opt. Spectrosc., vol. VII, no. 2, pp. 146-148, 1959.

[MOS77] M. Moszynski and B. Bengtson, "Light Pulse Shapes from Plastic Scintillators," Nucl. Instr. and Meth., vol. 142, pp. 417-434, 1977.

[MUR75] R. B. Murray, "Energy Transfer in Alkali Halide Scintillators by Electron-Hole Diffusion and Capture," IEEE Trans. Nucl. Sci., vol. 22, pp. 54-57, 1975.

[OHG92] H. Ohgaki, et al., "CsI(Tl) Yield Monitor for Quasi-Monochromatic Photons from Compton Backscattering of Laser Light at ETL," presented at the 1992 IEEE Nuclear Science Symposium and to appear in the Conference Record.

IOWE59] R. B. Owen, "Pulse-Shape Discrimination," Nucleonics, vol, 17, no. 9, pp. 92-95, 1959. 
[PEL76] J. P. Pellaux, "Determination des energies de migration thermique des centres $\mathrm{V}_{\mathrm{k}}$ dans Csl," Helv. Phys. Acta, vol. 49, pp. 7())-701, 1976.

[PIC66] H. Pick, "Luminescence of Anion Centres in Alkali Halides," Proc. Int. Conf. Lumin., pp. 659-679, 1966

[PLI59] 1. K. Pliavin', "On the Kinetics of Photo- and Gamma-Luminescence in Certain Thallium Activated Alkali-Halide Crystals," Opt. Spectrosc., vol. VII, no. 1, pp. 41-44, 1959.

[ROB59] J. C. Robertson and A. Ward, "Particle Selection in Crystals of CsI(Tl)," Proc. Phys. Soc., vol. 73, pp. 523-525, 1959.

[ROB61] J. C. Robertson and J. G. Lynch, "The Luminescent Decay of Various Crystals for Particles of Different Ionization Density," Proc. Phys. Soc., vol. 77. pp. 751-756, 1961.

[ROG75] D. W. O. Rogers, "Analytic and Graphical Methods for Assigning Errors to Parameters in Non-Linear Least Squares Fitting," Nucl. Instr. and Meth., vol. 127, pp. 253-260, 1975.

[SAK87] E. Sakai, "Recent Measurements on Scintillator-Photodetector Systems," IEEE Trans. Nucl. Sci., vol. 34, no. 1, pp. 418-422, 1987.

[SAS61] N. P. Sastry and B. V. Thosar, "Decay-Time of Scintillations in CsI(TI) Crystals," Proc. Ind. Acad. Sci., vol. 54A, pp. 14()-145, 1961.

[SCH90] P. Schotanus, R. Kamermans, and P. Dorenbos, "Scintillation Characteristics of Pure and Tl-Doped CsI Crystals," IEEE Trans. Nucl. Sci., vol. 37, no. 2, pp. 177-182, 1990.

[SE138] F. Seitz, "Interpretation of the Properties of Alkali Halide-Thallium Phosphors," J. Chem. Phys., vol. 6, pp. 150-162, 1938.

[SEI46] F. Seitz, "Color Centers in Alkali Halide Crystals," Rev. Mod. Phys., vol. 18. no. 3, pp. 384-408, 1946.

[SHA69] L. M. Shamovskii and A. D. Shushkanov, "Crystallochemical Laws of the Formation of Spectrometric Scintillators Based on Alkali-Halide Crystals," Sov. Phys. Dokl., vol. 13, no. 8, pp. 742-745, 1969.

[SON70] K. S. Song, "On the Migration of $\mathrm{V}_{k}$-centers in Alkali Halides," J. Phys. Chem. Solids, vol. 31, pp. 1389-1397, 1970.

[STO58] R. S. Storey, W. Jack, and A. Ward, "The Fluorescent Decay of CsI(Tl) for Particles of Different Ionization Density," Proc. Phys. Soc., vol. 72, pp. 1-8, 1958.

[STU87] A. Stuart and J. K. Ord, Kendall's Advanced Theory of Statistics. Volume L. Distribution Theory, 5th ed., Oxford University Press, New York, 1987.

[TOW68] T. Towyama, I. Morita, and M. Ishiguro, "The Luminescence in CsI(TI)," J. Phys. Soc. Japan, vol. 25, no. 4, pp. 1133-1139, 1968. 
|VAL92a| J. D. Valentine, V. T. Jordanov, D. K. Wehe, and G. F. Knoll, "Charge Calibration of CsI(TI)/Photodiode Spectroscopy Systens," Nucl. Instr. and Meth., vol. A314, pp. 119-124, 1992.

[VAL.92h] J. D. Valentine, R. F. Fleming, D. K. Wehe, and G. F. Knoll, "Uncertainty of Centroid and Full-width at Half Maximum Determinations and the Effect of Binning Data," presented at the 1992 IEEE Nuclear Science Symposium and to appear in the Conference Record.

[VAL93a] J. D. Valentine, W. W. Moses, S. E. Derenzo, D. K. Wehe, and G. F. Knoll, "Temperature Dependence of CsI(TI) Gamma-ray Ex ited Scintillation Characteristics," Nucl. Instr. and Meth, vol. A325, pp. 147-157, 1993.

[VAL.93b] J. Valentine, D. Wehe, G. Knoll, and C. Moss, "Temperature Dependence of CsI(Tl) Absolute Scintillation Yield", Conference Record of the 1991 IEEE Nuclear Science Symposium, vol. 1, pp. 176-182. Also submitted to IEEE Trans. Nucl. Sci.

[VAN56] W. Van Sciver, "Alkali Halide Scintillators," IRE Trans. Nucl. Sci., vol. 3, pp. 39-50, 1956.

[VAN57] W. J. Van Sciner and L. Bogart, "Conversion Efficiency of Scintillation Phosphors," Bull. Amer. Phys. Soc., ser. II, vol. 2, p. 142, 1957.

[VAN64] W. J. Van Sciver, "Role of the Thallium Dimer in the NaI(TI) Scintillation Process," Phys. Letters, vol. 9, no. 2, pp. 97-98. 1964.

[VIE74] W. Vichmann, J. F. Arens, and M. Simon, "Optical Transmission Measurements on Monocrystalline and Polycrystalline Cesium Iodide," Nucl. Instr. and Meth., vol. 116, pp. 283-295, 1974.

[VIE86] G. Viesti, G. Prete, D. Fabris, K. Hagel, G. Nehbia, and A. Menchaca-Rocha, "The Response of a CsI(TI) Scintillator with Photodiode Readout to Light Particles and Heavy Ions," Nucl. Instr. and Meth., vol. A252, pp. 75-79, 1986.

|VIT66] I. Vitols, "Radiative Recombination of Point Microdefects (Defectons) in Alkali Halide Crystals," Proc. Int. Conf. Lumin., pp. 866-872, 1966. 

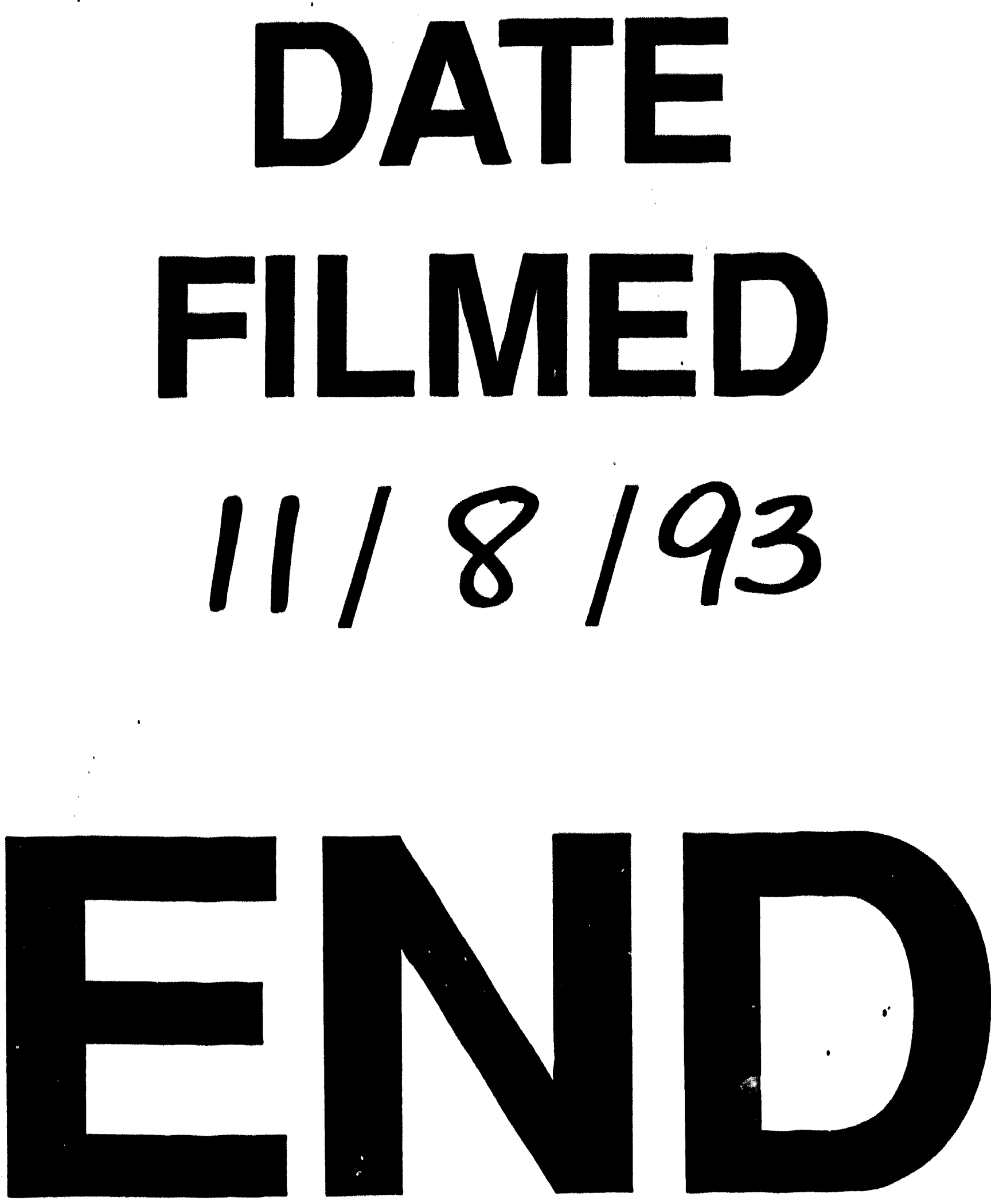
